JORGE ANDRÉS JULCA AVILA

SOLUÇÃO NUMÉRICA EM JATOS DE LÍQUIDOS METAESTÁVEIS COM EVAPORAÇÃO RÁPIDA

Tese apresentada à Escola

Politécnica da Universidade de São

Paulo para obtenção do título de Doutor em Engenharia 
JORGE ANDRÉS JULCA AVILA

\section{SOLUÇÃO NUMÉRICA EM JATOS DE LÍQUIDOS METAESTÁVEIS COM EVAPORAÇÃO RÁPIDA}

Tese apresentada à Escola Politécnica da Universidade de São Paulo para obtenção do título de Doutor em Engenharia

Área de concentração:

Engenharia Mecânica

Orientador:

Prof. Dr. Marcos de Mattos Pimenta 
Este exemplar foi revisado e alterado em relação à versão original, sob responsabilidade única do autor e com anuência de seu orientador.

São Paulo, 16 de junho de 2008

Assinatura do autor

Assinatura do orientador

Julca Avila, Jorge Andrés

Solução numérica em jatos de líquidos metaestáveis com evaporação rápida / J.A.J. Avila. -- São Paulo, 2008.

p. 144

Tese (Doutorado) - Escola Politécnica da Universidade de São Paulo. Departamento de Engenharia Mecânica.

1. Evaporação rápida 2. Escoamento bifásico 3. Ondas de choque 4. Esquema de MacCormack 5. Lei de conservação I. Universidade de São Paulo. Escola Politécnica. Departamento de Engenharia Mecânica II. t. 


\section{DEDICATÓRIA}

Dedico esta tese aos meus pais, a minha esposa e filho e a meus irmãos.

Confia no SEJNHOR de todo o teu coração e não te estribes no teu próprio entendimento.

Reconhece-o em todos os teus caminhos, e ele endireitará as tuas veredas.

Não sejas sábio aos teus próprios olhos; teme ao SENHHOR e aparta-te do mal; será isto saúde para o teu corpo e refrigério, para os teus ossos.

Honra ao SENHHOR com os teus bens e com as primícias de toda a tua renda; e se encherão fartamente os teus celeiros, e transbordarão de vinho os teus lagares.

(Pravérlias 3:5-10) 


\section{AGRADECIMENTOS}

O primeiro gesto de agradecimento é a Deus - d'Ele que todas as coisas dependem - e que, com a sua infinita bondade, concedeu-me a oportunidade de, na difícil caminhada, experimentar a inefável sensação de alcançar um patamar acima, o meu doutorado.

Depois, o agradecimento aos meus pais que, na segurança de um lar bem estruturado, plasmaram a minha personalidade e fincaram a marcação do meu caráter.

Logo a seguir, acode-me a imagem da minha doce companheira, Elizangela, sempre acreditando em mim, impelindo-me a constantes buscas de aperfeiçoamento, inculcando-me o salutar desejo de superar as minhas próprias e humanas limitações.

Ao meu orientador, Dr. Marcos de Mattos Pimenta, a quem devoto a mais sincera e efusiva admiração por acreditar neste trabalho, pelo acompanhamento e diretrizes em todas as suas etapas e pelo convívio acadêmico de mais de quatro anos.

Ao Professor Dr. José Roberto Simões Moreira, pelos ensinamentos transmitidos, apoio e incentivo na realização deste trabalho.

Aos membros da banca examinadora pelas opiniões valiosas e preciosas sugestões.

Aos meus colegas: Marcelo Rocha, Ricardo, André e Elí do laboratório SISEA e a Marcelo Mendes ex-Siseano, pela amizade e incentivo em todos os momentos.

Agradeço a meu irmão Juan, pela compreensão e apoio moral, e ainda, a alguns amigos, Jaime, Juan Carlos, Valentin, Nestor e Christian.

Finalmente, ao CNPq, pelo apoio financeiro proporcionado durante o desenvolvimento desta tese. 


\section{RESUMO}

Este trabalho estuda o fenômeno de evaporação rápida em jatos de líquidos superaquecidos ou metaestáveis numa região 2D. O fenômeno se inicia, neste caso, quando um jato na fase líquida a alta temperatura e pressão, emerge de um diminuto bocal projetando-se numa câmara de baixa pressão, inferior à pressão de saturação. Durante a evolução do processo, ao cruzar-se a curva de saturação, se observa que o fluido ainda permanece no estado de líquido superaquecido. Então, subitamente o líquido superaquecido muda de fase por meio de uma onda de evaporação oblíqua. Esta mudança de fase transforma o líquido superaquecido numa mistura bifásica com alta velocidade distribuída em várias direções e que se expande com velocidades supersônicas cada vez maiores, até atingir a pressão a jusante, e atravessando antes uma onda de choque. As equações que governam o fenômeno são as equações de conservação da massa, conservação da quantidade de movimento, e conservação da energia, incluindo uma equação de estado precisa. Devido ao fenômeno em estudo estar em regime permanente, um método de diferenças finitas com modelo estacionário e esquema de MacCormack é aplicado. Tendo em vista que este modelo não captura a onda de choque diretamente, um segundo modelo de falso transiente com o esquema de "shock-capturing": "Dispersion-Controlled Dissipative" (DCD) é desenvolvido e aplicado até atingir o regime permanente.

Resultados numéricos com o código ShoWPhasT-2D v2 e testes experimentais foram comparados e os resultados numéricos com código $D C D-2 D$ v1 foram analisados.

Palavras-chave: Evaporação rápida ("flashing"). Escoamento bifásico. Onda de choque. Sistema de Leis de conservação. Esquema de MacCormack. Esquema "Dispersion-Controlled Dissipative" (DCD). Esquemas de "shock-capturing". 


\begin{abstract}
This study analyses the rapid evaporation of superheated or metastable liquid jets in a two-dimensional region. The phenomenon is triggered, in this case, when a jet in its liquid phase at high temperature and pressure, emerges from a small aperture nozzle and expands into a low pressure chamber, below saturation pressure. During the evolution of the process, after crossing the saturation curve, one observes that the fluid remains in a superheated liquid state. Then, suddenly the superheated liquid changes phase by means of an oblique evaporation wave. This phase change transforms the liquid into a biphasic mixture at high velocity pointing toward different directions, with increasing supersonic velocity as an expansion process takes place to the chamber back pressure, after going through a compression shock wave. The equations which govern this phenomenon are: the equations of conservation of mass, momentum and energy and an equation of state. Due to its steady state process, the numerical simulation is by means of a finite difference method using the McCormack method of Discretization. As this method does not capture shock waves, a second finite difference method is used to reach this task, the method uses the transient equations version of the conservation laws, applying the Dispersion-Controlled Dissipative (DCD) scheme.

Numerical results using the code ShoWPhasT-2D v2 and experimental data have been compared, and the numerical results from the $D C D-2 D v 1$ have been analysed.
\end{abstract}

Keywords: Rapid evaporation (flashing). Two-phase flow. Shock wave. System of conservation Laws. MacCormack scheme. Dispersion-Controlled Dissipative (DCD) scheme. shock-capturing Schemes. 


\section{SUMÁRIO}

$\begin{array}{ll}\text { LISTAS DE FIGURAS } & \text { vii } \\ \text { LISTAS DE TABELAS } & \text { ix } \\ \text { LISTAS DE SÍMBOLOS } & \mathbf{x}\end{array}$

1. INTRODUÇÃO

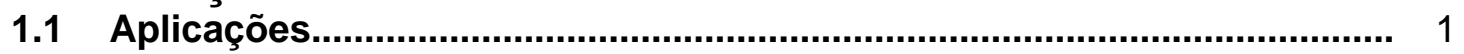

1.1.1 Acidentes industriais............................................................................ 1

1.1.2 Injeção de combustível....................................................................... 2

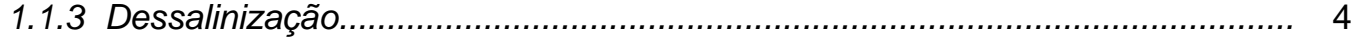

1.1.4 Válvulas de segurança e de alivio.......................................................... 4

1.1.5 Dispositivos de expansão.................................................................... 5

1.2 Descrição do Problema............................................................................... 6

1.3 Revisão Bibliográfica............................................................................. 7

1.3.1 Fenômenos evaporativos........................................................................... 7

1.3.2 Jatos de líquidos superaquecidos: Onda de choque.................................. 9

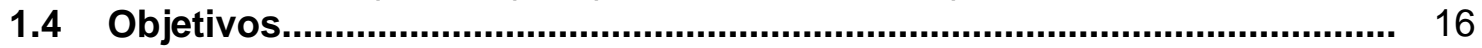

1.5 Divisão da Tese......................................................................................... 17

2. ESTADOS TERMODINÂMICOS DA PRÉ-EXPANSÃO BIFÁSICA 19

2.1 Estado Inicial.................................................................................... 21

2.2 Estado Metaestável.................................................................................... 22

2.2.1 Pressão metaestável...................................................................................... 22

2.2.2 Volume específico metaestável............................................................. 23

2.3 Estado Pós-onda de Evaporação................................................................... 24

2.3.1 Notações e hipóteses simplificadoras........................................................ 25

2.3.2 Equações de salto.................................................................................... 28

2.3.3 Equações de Rayleigh e Rankine-Hugoniot................................................ 32

2.3.4 Solução de Chapman-Jouguet (C-J)..................................................... 32

2.4 Ângulos de Onda e de Giro...................................................................... 35

2.4.1 Ângulo de onda................................................................................. 35

2.4.2 Ângulo de giro............................................................................ 37

2.5 Formato do Núcleo Líquido........................................................................ 39

2.5.1 Novo raio do bocal.......................................................................... 39

3. EXPANSÃO BIFÁSICA 44

3.1 Domínio Físico.......................................................................................... 44

3.2 Domínio Computacional................................................................................ 45

3.3 Condições de Contorno.............................................................................. 46

3.4 Geração de Malha Estruturada.............................................................. 47

3.4.1 Transformação de coordenadas........................................................... 47

3.4.2 Malha física e computacional......................................................................... 49

3.5 Formulação Matemática das Equações Governantes............................... 49

3.5.1 Em coordenadas cartesianas............................................................... 49

3.5.2 Em coordenadas curvilíneas ortogonais................................................... 51

4. SOLUÇÃO NUMÉRICA: MÉTODO ESTACIONÁRIO 58

4.1 Equação Governante.................................................................................. 58

4.2 Método de MacCormack.......................................................................... 59

4.3 Viscosidade Artificial.......................................................................... 60

4.4 Decodificação nas Variáveis Primitivas.................................................. 61

4.5 Captura da Onda de Choque................................................................. 62

4.6 Refinamento da Malha......................................................................... 63 
5. SOLUÇÃO NUMÉRICA: MÉTODO NÃO ESTACIONÁRIO 66

5.1 Autovalores das Matrizes Jacobianas.................................................... 66

5.1.1 Autovalores no sistema de coordenadas cartesianas.................................. 67

5.1.2 Autovalores no sistema de coordenadas curvilíneas ortogonais...................... 69

5.2 Esquema "Dispersion-Controlled Dissipative" DCD................................. 72

5.2.1 Formulação por diferenças finitas............................................................ 72

5.2.2 Discretização temporal......................................................................... 75

5.2.3 Domínio computacional...................................................................... 76

6. RESULTADOS NUMÉRICOS: CASO ESTACIONÁRIO 78

6.1 Condição de Contorno na Linha inicial.................................................. 78

6.2 Variação da Pressão na Câmara de Injeção............................................. 79

6.3 Refinamento na Malha....................................................................... 81

6.4 Comparação com Resultados Experimentais......................................... 82

6.4.1 Variação das condições de reservatório..................................................... 82

6.4.2 Localização da onda de choque................................................................ 85

6.5 Comparação com Resultados Numéricos............................................... 85

6.5.1 Condições de contorno............................................................................... 86

6.5.2 Tempo computacional............................................................................. 87

7. RESULTADOS NUMÉRICOS: CASO FALSO TRANSITÓRIO 89

7.1 Validação do Código Computacional DCD-2D v1..................................... 89

7.1.1 Reflexão de uma onda de choque.......................................................... 90

7.1.2 Bocal convergente-divergente................................................................... 95

7.1.3 Bocal supersônico..................................................................................... 98

7.1.4 Jato evaporativo..................................................................................... 100

7.2 Expansão Bifásica.......................................................................... 103

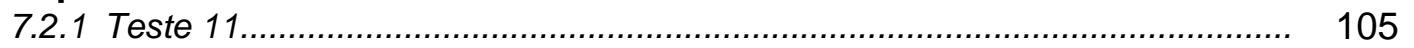

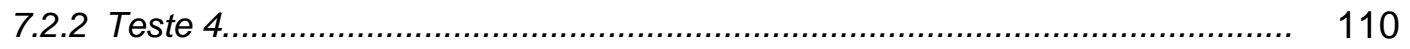

7.2.3 Variação da pressão ao longe..................................................................... 113

7.2.4 Independência da malha............................................................................. 114

7.2.5 Comparação com resultados experimentais................................................. 114

8. CONCLUSÕES E CONTINUIDADE DO TRABALHO 116

8.1 Conclusões.................................................................................. 116

8.2 Continuidade do Trabalho................................................................... 118

$\begin{array}{ll}\text { REFERÊNCIAS BIBLIOGRÁFICAS } & 119\end{array}$

APÊNDICE A - ALGORITMOS EM PSEUDOCÓDIGO

APÊNDICE B - CONDIÇÕES DE CONTORNO

APÊNDICE C - CÁLCULO DA MATRIZ JACOBIANA 132

APÊNDICE D - EQUAÇÃO DE ESTADO DE LEE-KESLER 138 


\section{LISTA DE FIGURAS}

Figura 1.1 Diferentes quadros de ondas de choques através do tempo, MacPhee et al. (2002).

Figura 1.2 Ondas de evaporação (SIMÕES-MOREIRA et al., 2003), em: (a) Tubo capilar. (b) Válvula de expansão

Figura 1.3 Arranjo experimental para observar ondas de evaporação (SIMÕESMOREIRA et al.,1993).....

Figura 1.4 Fotografia de uma onda de evaporação em progresso do dodecano superaquecido no interior de um tubo de vidro. A temperatura é de $230^{\circ} \mathrm{C}$, a pressão acima de onda de evaporação é de 0.37 bar e abaixo da onda é de 0.59 bar, e sua velocidade é de $47.2 \mathrm{~cm} / \mathrm{s}$ (SIMÕESMOREIRA, 1994).

Figura 1.5 Jato de diferentes geometrias e graus crescentes de superaquecimento $H$ e com fluido teste perfluor-n-hexano (KURSCHAT et al., 1992)

Figura 1.6 Imagem do jato de iso-octano com temperatura e pressão de injeção de $T_{0}=223^{\circ} \mathrm{C}$ e $P_{0}=2068 \mathrm{kPa}$, obtidas pelo método de "laser sheet" (ATHANS, 1995).

Figura 1.7 Seqüência de imagens de jatos evaporativos de querosene com pressão de injeção de $600 \mathrm{kPa}$, e pressão da câmara igual a $1.6 \mathrm{kPa}$. A Temperatura de injeção é de $38,95,120$ e $260^{\circ} \mathrm{C}$, respectivamente (VIEIRA, 1999).

Figura 1.8 Resultados numéricos de Angelo (2004). Localização da Onda de Choque onde a pressão na câmara de injeção é de $90 \mathrm{~Pa}$. (a) Fotografia de Vieira (2005). (b) Pressão. (c) Temperatura. (d) Título. (d) Número de Mach.

Figura 1.9 Esquema geral da bancada experimental, Vieira (2005)

Figura 1.10 Ondas de choques (Ensaio 5435m10c; Vieira, 2005): a) Imagem original do ensaio. b) Imagem tratada com filtro matemático. c) Imagem processada para destacar o núcleo do líquido e as ondas de choques.

d) Fotografia obtida com o método de iluminação por atrás.

Figura 2.1 Organograma da divisão dos Estados Termodinâmicos que envolvem o fenômeno.

Figura 2.2 Representação dos estados termodinâmicos nos jatos evaporativos altamente superaquecidos. (a) Fotografia de Vieira (2005). (b) Representação esquemática do fenômeno. (c) Comportamento termodinâmico do fenômeno no diagrama $P-v$

Figura 2.3 Onda de descontinuidade em um volume de controle e propriedades à montante e à jusante da descontinuidade. 
Figura 2.4 Comportamento esquemático do estado à montante de uma onda de evaporação (Simões-Moreira, 1994)...

Figura 2.5 Formato do núcleo líquido cônico. (a) Superfície do núcleo líquido em 3D. (b) Secção longitudinal de rotação do cone ao redor do eixo "Z".......

Figura 2.6 Ângulo de giro em uma descontinuidade. (a) Ângulo de giro para uma onda de evaporação. (b) Ângulo de giro para uma onda de choque.

Figura 2.7 Foto do núcleo líquido cônico da Serie RUN3, ensaio 7404500, Vieira (2005)

Figura 2.8 Formato esquemático do novo núcleo líquido quase-paraboloidal. (a) Superfície do núcleo líquido. (b) Secção longitudinal que gera o formato quase-paraboloidal do núcleo líquido.

Figura 3.1 Domínio da expansão bifásica. a) Domínio físico $\Gamma$ de raio $R_{f}$. b) Domínio computacional $\Gamma^{\prime}$

Figura 3.2 (a) Malha Física de raio $R_{f}$. (b) Malha Computacional.

Figura 3.3 Transformação de coordenadas

Figura 4.1 Célula padrão da malha. (a) Célula do domínio computacional. (b) Célula do domínio físico

Figura 4.2 (a) Malha. (b) Malha-i. (c) Pré-malha refinada. (d) Malha-j. e) Malha refinada.

Figura 5.1 Célula computacional para determinar a variação dos fluxos numéricos.

Figura 5.2 Nós do domínio computacional.

Figura 6.1 Ondas de choques variando a pressão na câmara de injeção.

Figura 6.2 Onda de choque obtida numericamente. a) Sem refinamento. b) Com refinamento

Figura 6.3 Onda de choque obtida experimentalmente (Serie RUN 2, ensaio 5393m10b, imagem 2), segundo Vieira (2005). a) Foto original. b) Imagem realçada.....

Figura 6.4 Dimensões da onda de choque para o caso sem refinamento num total de 20 testes. a) Comprimento radial, $r_{1}[\mathrm{~mm}]$. b) Comprimento axial, $r_{2}[\mathrm{~mm}]$

Figura 6.5 Localização da onda de choque em: (a) Fotografia de Vieira (2005); (b) Pressão; (c) Temperatura; (d) Título; (e) Número de Mach. 
Figura 6.6 Linha de corrente e onda de choque para o Teste 10 (Serie RUN3, ensaio 5329500, $P_{0}=497,6[\mathrm{kPa}] ; T_{0}=76,9\left[{ }^{\circ} \mathrm{C}\right] ; P_{\infty}=110[\mathrm{~Pa}]$ ). (a) Resultado com o código ShoWPhasT-2D v1, Angelo (2004). (b) Resultado deste trabalho com o código ShoWPhasT-2D v2. (c) Resultado experimental, Vieira (2005).

Figura 7.1 Reflexão de uma onda de choque

Figura 7.2 Reflexão de uma onda de choque. (a) Geometria com malha e a linha fixa $\overline{A B}$. (b) Distribuição de pressões. (c) Distribuição de temperaturas. (d) Número Mach.

Figura 7.3 (a) Comportamento da pressão versus número de iterações. Os seguintes gráficos: (b) Pressão, (c) Temperatura e (d) Número Mach atingiram o regime permanente.

Figura 7.4 Bocal convergente-divergente. (a) Geometria com malha. (b) Distribuição de pressões. (c) Distribuição de temperaturas. (d) Número Mach.

Figura 7.5 (a) Comportamento da pressão versus número de iterações para um nó fixo da malha. Os seguintes gráficos: (b) Pressão, (c) Temperatura e (d) Número Mach atingiram o regime permanente e indicam sua distribuição espacial ao longo de uma linha fixa da malha.

Figura 7.6 Bocal supersônico. (a) Geometria com malha. (b) Distribuição de pressões. (c) Distribuição de temperaturas. (d) Número Mach.

Figura 7.7 (a) Comportamento da pressão versus número de iterações para um nó fixo da malha. Os seguintes gráficos: (b) Pressão, (c) Temperatura e (d) Número Mach atingiram o regime permanente e indicam sua distribuição espacial ao longo de uma linha fixa da malha.

Figura 7.8 Jato evaporativo. (a) Geometria com malha. (b) Distribuição de pressões. (c) Distribuição de temperaturas. (d) Número Mach......

Figura 7.9 (a) Comportamento da pressão versus número de iterações para um nó fixo da malha. Os seguintes gráficos: (b) Pressão, (c) Temperatura e (d) Número Mach atingiram o regime permanente e indicam sua distribuição espacial ao longo de uma linha fixa da malha.

Figura 7.10 Jato supersônico. (a) Geometria com malha. (b) Distribuição de pressões. (c) Distribuição de temperaturas. (d) Número Mach. (e) Título. (f) Densidade.

Figura 7.11 (a) Comportamento da pressão versus número de iterações para um nó fixo da malha. Os seguintes gráficos: (b) Pressão, (c) Número Mach e (d) Título atingiram o regime permanente e indicam sua distribuição espacial ao longo de uma linha fixa da malha.

Figura 7.12 Distribuição do gráfico da pressão em 3D.

Figura 7.13 Domínio não-simétrico. a) Distribuição da pressão b) Distribuição do número Mach. 
Figura 7.14 Jato supersônico. (a) Geometria com malha. (b) Distribuição de pressões. (c) Distribuição de temperaturas. (d) Número Mach. (e) Título. (f) Densidade.

Figura 7.15 (a) Comportamento temporal da pressão versus número de iterações para um nó fixo da malha. Os seguintes gráficos: (b) Pressão, (c) Número Mach e (d) Título atingiram o regime permanente e indicam sua distribuição espacial ao longo de uma linha fixa da malha.

Figura 7.16 Variação da pressão ao longe e dimensão da onda de choque.

Figura A.1 Algoritmo em pseudocódigo para obter o estado metaestável.

Figura A.2 Algoritmo em pseudocódigo para achar as propriedades à jusante da onda de evaporação.

Figura A.3 Algoritmo em pseudocódigo da Derivada_Dp_Drho.

Figura A.4 Algoritmo em pseudocódigo do código computacional $D C D-2 D$ v1.

Figura D.1 Algoritmo em pseudocódigo da sub-rotina Interpolação de Lee-Kesler.... 


\section{LISTA DE TABELAS}

Tabela 6.1 Estados Termodinâmicos da Pré-expansão Bifásica e Pós-onda de Evaporação para $R_{b}=0,1530[\mathrm{~mm}]$ e $\left\{P_{0}, T_{0}\right\}$ conforme a (Vieira, 2005).

Tabela 6.2 Resultados Experimentais e Numéricos: Variação das condições de reservatório.

Tabela 6.3 Erro relativo da dimensão da onda de choque entre os resultados numéricos e experimentais (Vieira, 2005)

Tabela 6.4 Tempo CPU de execução do código

Tabela 7.1 Condições de contorno para o problema de reflexão da onda de choque.

Tabela 7.2 Solução analítica e numérica da reflexão da onda de choque.....

Tabela 7.3 Condições de contorno para o bocal convergente-divergente......

Tabela 7.4 Solução do bocal convergente-divergente

Tabela 7.5 Solução do bocal supersônico

Tabela 7.6 Bocal supersônico com vetor velocidade oblíquo.......

Tabela 7.7 Grupo de malhas refinadas, $R_{f}=22[\mathrm{~mm}]$

Tabela 7.8

Solução numérica do Teste $11 .$.

Tabela 7.9 Solução numérica para o caso sem condição de simetria.

Tabela 7.10

Solução numérica do Teste 4 .......

Tabela 7.11

Variação da pressão ao longe.

Tabela 7.12 Independência da malha.

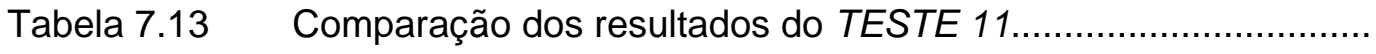

Tabela 7.14 Comparação dos resultados do TESTE 04..

Tabela A.1 Sub-rotinas e funções..

Tabela B.1 Valores de contorno na Linha inicial......

Tabela B.2 Valores de contorno na Linha de simetria.

Tabela B.3 Valores de contorno na Linha ao longe. 
Tabela B.4 Valores de contorno na Linha de parede.................................. 131

Tabela D.1 Propriedades do iso-octano (Reid et al. 1987) .......................... 139

Tabela D.2 Constantes da Equação de Lee-Kesler.................................... 140

Tabela D.3 Sub-rotina para o cálculo da Equação de Estado de Lee-Kesler para o estado (1)......................................................... 141

Tabela D.4 Tabela de propriedades de saturação do iso-octano $\left(C_{8} H_{18}\right) \ldots \ldots \ldots . \quad 142$

Tabela D.5 Sub-rotina: Interpolação_Lee-Kesler......................................... 143

Tabela D.6 Sub-rotinas que compõem a rotina: Interpolação_Lee-Kesler..... 143 


\section{LISTA DE SÍMBOLOS}

$a_{m}=$ Tangente da geratriz do tronco de cone

$A_{b}=$ Área da seção de saída do bocal de injeção

$A_{n b}=$ Área da seção de saída do novo bocal de injeção

$C_{D}=$ Coeficiente de descarga

$C_{x}=$ Parâmetro adimensional de viscosidade artificial

$e=$ Energia interna específica

$h=$ Entalpia específica

$h_{0} \quad=\quad$ Entalpia específica total

$J \quad=\quad$ Fluxo de massa por unidade de área

$J_{a}=$ Matriz Jacobiana

$L_{A E}=$ Altura do troco de cone

$L_{E} \quad=\quad$ Comprimento do núcleo líquido metaestável

$L_{N}=$ Comprimento do novo núcleo líquido metaestável

$\dot{m}=$ Vazão em massa

$M \quad=\quad$ Número de Mach

$\vec{n} \quad=\quad$ Vetor normal exterior unitário

$P=$ Pressão

$r=$ Coordenada radial

$r_{1}=$ Dimensão máxima da onda de choque na direção radial

$r_{2}=$ Dimensão máxima da onda de choque na direção axial

$R_{b} \quad=\quad$ Raio do bocal de injeção

$R_{f} \quad=\quad$ Raio da geometria bifásica

$R_{n b} \quad=\quad$ Raio do novo bocal de injeção

$s=$ Entropia específica

$S=$ Termo fonte

$S=$ Fronteira de Controle

$\vec{t}=$ Vetor tangencial unitário

$T=$ Temperatura 


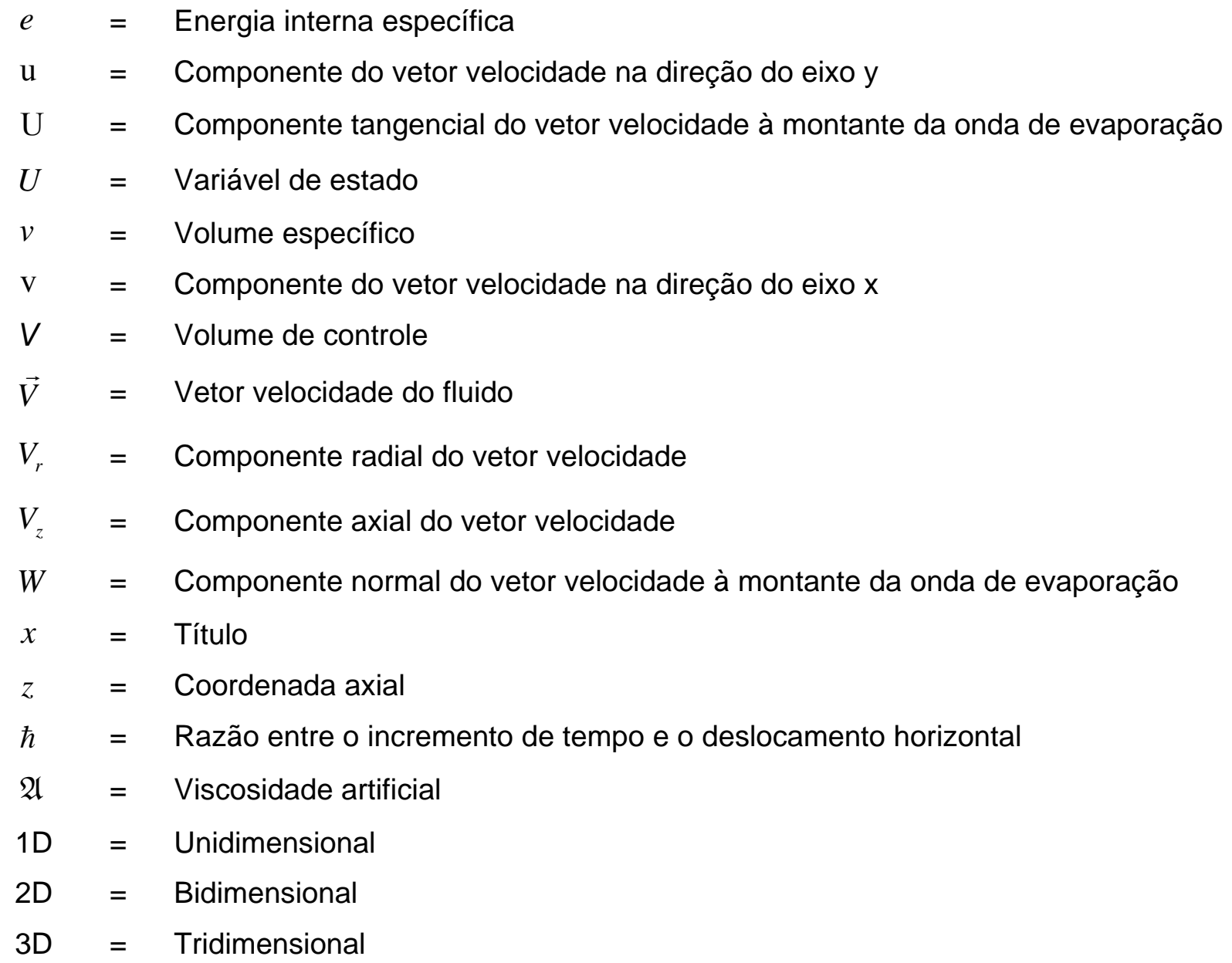

Gregos

$\beta=$ Ângulo de onda

$\varepsilon=$ Energia total específica

$\eta=$ Coordenada vertical no domínio computacional

$\theta=$ Ângulo de giro

$\rho=$ Densidade

$\tau=$ Coordenada temporal no domínio computacional

$\tau=$ Tensor dos esforços viscosos

$\xi=$ Coordenada horizontal no domínio computacional

$\Phi=$ Função de fluxo

$\Gamma=$ Fronteira do domínio físico

$\Lambda_{A}=$ Vetores que consistem dos autovalores da matriz $A$

$\Upsilon=$ Fronteira do domínio computacional

$\Psi=$ Função de Fluxo 
Subscritos

1 = Estado metaestável ou superaquecido ou à montante da onda de evaporação

$2=$ Estado pós-onda de evaporação ou à jusante da onda de evaporação

$3=$ À montante da onda de choque

$4=$ À jusante da onda de choque

$\infty=$ Câmara de injeção

$\sigma=$ Propriedade saturada 


\section{INTRODUÇÃO}

\subsection{Aplicações}

O fenômeno de evaporação rápida em jato de líquidos metaestáveis é importante em aplicações industriais e tecnológicas. Diversas pesquisas teóricas, experimentais e computacionais têm sido desenvolvidas de forma abrangente no intuito de conhecer e prever o comportamento dos fluidos durante o processo de mudança de fase de líquido para vapor. São de interesse específico nesta área os modelos matemáticos e os códigos computacionais preditivos. Espera-se que esses modelos incorporem suficiente compreensão destes fenômenos, e que suas simulações numéricas sejam adequadas para resolver problemas de engenharia. Algumas aplicações deste fenômeno estão presentes nos acidentes industriais, injeção de combustível, dessalinização, válvulas de segurança e alivio e dispositivos de expansão. A seguir descreveremos cada uma dessas aplicações.

\subsubsection{Acidentes industriais}

A indústria desempenha um papel preponderante no desenvolvimento da sociedade. A maior parte dos produtos ou materiais que usamos em nosso dia a dia é produzida pela indústria química. No desenvolvimento destas e de novos materiais aparecem situações que precisam ser bem analisadas para redução do risco de acidentes.

\section{- LOCA}

Fenômenos de evaporação rápida podem ser perigosos em algumas situações. O acidente de perda de refrigerante ("Loss of Cooling Accidents” ou LOCA) tem sido estudado extensivamente em análise de segurança em plantas de potência nuclear. Neste acidente, águas quentes sob pressão em linhas resfriadas são expostas subitamente à atmosfera através de uma falha de tubulação ou válvulas. A queda de pressão resulta em superaquecimento e subsequentemente no "flashing” da água que força violentamente um escoamento bifásico através de uma ruptura. Problemas similares podem ocorrer em encanamento de gases liquefeitos ou reatores químicos, Alamigir et al. (1981). 


\section{- BLEVE}

Outro perigoso cenário é o líquido em ebulição expandindo via explosão de vapor (“Boiling-Liquid, Expanding-Vapor Explosion” ou BLEVE). Refere-se a uma explosão física onde os perigos são ondas expansivas e projéteis. Sabe-se que uma substância de embarcação não necessariamente deve ser inflamável para produzir uma BLEVE. Nestes incidentes, o fogo a redor de um container cheio de líquido causa ebulição e faz a pressão interna do container subir. Embora a maioria de embarcações que carregam gases liquefeitos tem válvulas de alívio que se ativam quando certas pressões são atingidas, elas são frequentemente incapazes de liberar o vapor rápido o suficiente para reduzir a pressão interna. Em conseqüência o container estoura e o líquido restante ferve de maneira explosiva. Se o fluido é inflamável, a liberação pode ser inflamada causando uma bola de fogo, Prugh (1991).

\section{- $\quad P L G$}

Muitos materiais perigosos são armazenados e transportados na forma líquida. As condições termodinâmicas requeridas para permitir o armazenamento de uma substância numa determinada fase dependem da temperatura de ebulição e da temperatura crítica da substância. Líquidos armazenados em temperatura ambiente que sejam maior que de sua ebulição atmosférica e menor que sua temperatura crítica, são líquidos que passam pelo processo de "flashing”. Para a liquefação destas substâncias, elas devem ser comprimidas a partir de seu estado gasoso atmosférico. Portanto estas substâncias são referidas como gases liquefeitos pressurizados ("Pressured Liquefied Gases" ou PLG). A parte explosiva da liberação é causada por uma vaporização do líquido extremamente rápido e muitas vezes exibem formação de ondas de choques, Ogle et al. (2004).

\subsubsection{Injeção de combustível}

A compreensão do fenômeno e da forma como o combustível se comporta é crucial para a fabricação de motores mais eficientes e menos poluidores. MacPhee et al. (2002) detectaram a existência de uma onda de choque supersônica no interior de uma câmara de combustão de motores a diesel. A forma tradicional de se estudar o padrão de dispersão do diesel no ar utiliza feixes de luz projetados sobre o jato de combustível que sai do bico injetor. Mas a luz acaba por ser refletida por inúmeras gotículas, fazendo com que o resultado observado não 
seja muito fiel. Para uma melhor visualização do fenômeno, Macphee et al. (2002) empregou raio-X e um detector de alta velocidade, capaz de gravar uma imagem a cada cinco microssegundos. O diesel injetado recebeu uma mistura de compostos de césio para melhorar o contraste com os raios X. A câmara de combustão foi previamente preenchida com hexafluoreto de enxofre $\left(S F_{6}\right)$, para evitar a combustão. Os pesquisadores descobriram que 90\% do combustível concentram-se em um fino jato que fica atrás da onda de choque, que tem o formato do cone de Mach. A maior concentração de combustível vem logo atrás da parte frontal da onda. Enquanto o gás na câmara de combustão freia a parte frontal do jato de combustível, a porção posterior do jato move-se várias vezes mais rápidas, a uma velocidade supersônica. Como a parte final do jato de combustível alcança a parte da frente, a maior parte do diesel fica concentrada em uma espécie de gota logo atrás do ponto de choque. Na Fig. 1.1 podemos observar imagens da radiografia em função do tempo, de um “spray” de combustível e ondas de choques geradas pelo "spray" em instantes de tempo de 38, 77, 115, 154, e 192 microssegundos depois de iniciado a injeção (escolhido de um total de 168 quadros). Para otimizar as condições de uma visualização direta das ondas de choques, a pressão escolhida foi de $135 \mathrm{MPa}$.

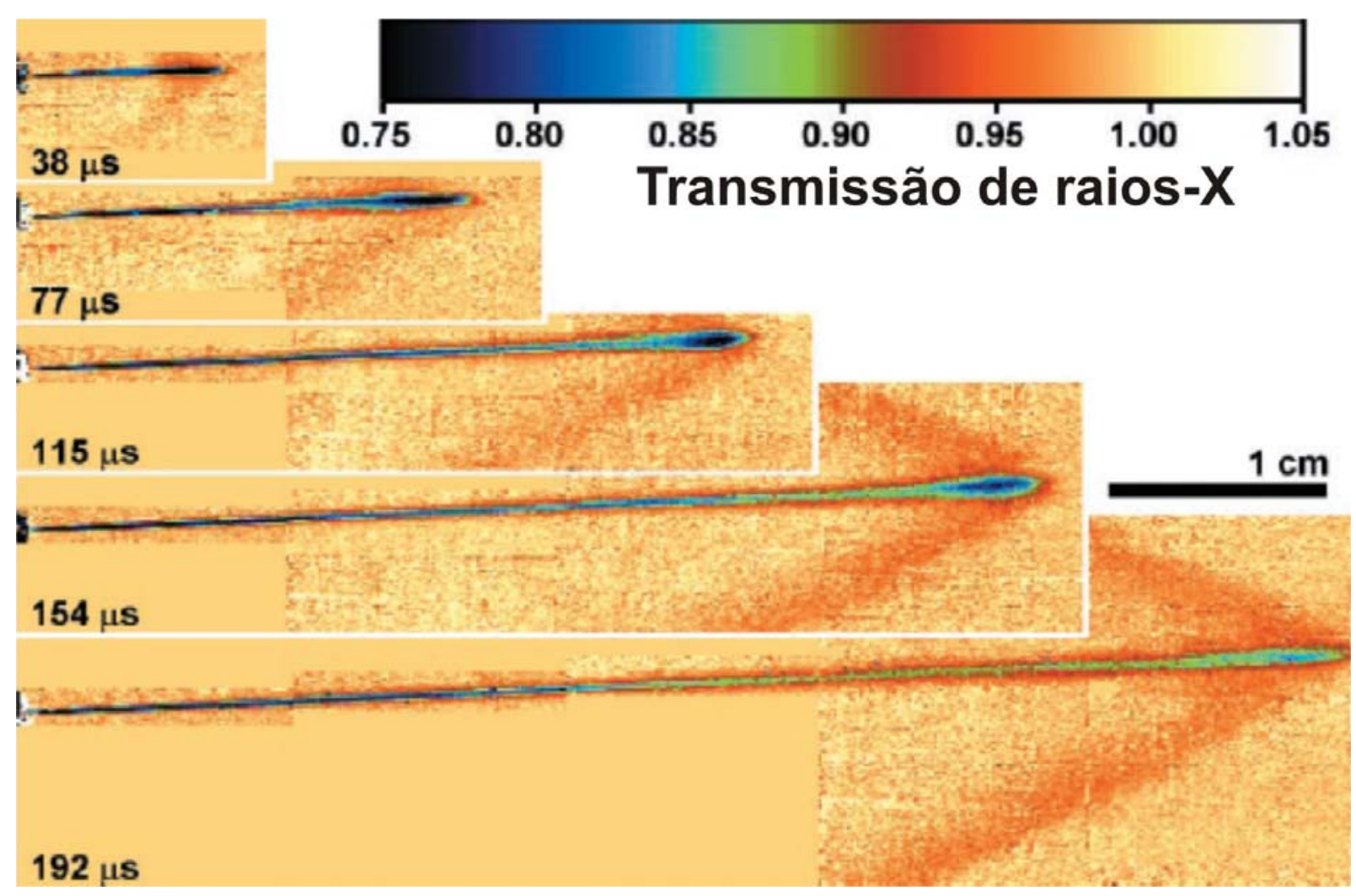

Figura 1.1 Diferentes quadros de ondas de choques através do tempo, MacPhee et al. (2002). 


\subsubsection{Dessalinização}

A dessalinização é o processo de transformar água salgada ou salobra em água potável, é freqüentemente apontada como a grande solução para o fornecimento de água potável para várias partes do mundo num futuro próximo. O problema é que as tecnologias atuais são vorazes consumidoras de energia.

Devido à escassez da água em muitos lugares do mundo, alguns paises foram forçados a obter água do mar como fonte alternativa. Muitas plantas de dessalinização estão sendo instaladas no mundo inteiro, sendo que mais da metade são instaladas no meio Oriente e o norte da África. Estados Unidos encontra-se em segundo lugar da capacidade instalada, Kahraman e Cengel (2005).

O processo predominante de dessalinização de água de mar é a Destilação multi-etapa em Flash (“Multi-Stage Flash” ou MSF), ver Al-Hengari et al. (2005). O princípio em que se baseia este processo é o de que a temperatura para evaporação da água depende da pressão existente à superfície desta água. A água do mar é aquecida numa câmara chamada aquecedor de salmoura até uma determinada temperatura sob uma pressão suficientemente alta para que se evite sua ebulição. Essa água aquecida flui para uma outra câmara, chamado estágio ("flash chamber”), onde a pressão ambiente é tal que a água irá se vaporizar instantaneamente (flash). A solução restante passa para a câmara seguinte, onde existe um vácuo mais elevado do que da primeira câmara. A salmoura vai passando sucessivamente para as câmaras seguintes, sob vácuos sucessivamente mais elevados. Tipicamente, uma planta de destilação flash pode conter de 4 a 40 estágios. O vapor gerado em cada estágio é convertido em água destilada por condensação sobre tubos trocadores de calor, que correm através de cada estágio. Os tubos são resfriados pela água de alimentação que está indo para o aquecedor de salmoura.

\subsubsection{Válvulas de segurança e de alívio}

A válvula de segurança é a que protege um equipamento. A função de toda válvula de segurança instalada em processos indústrias é aliviar o excesso de pressão, devido ao aumento da pressão de operação acima de um limite pré-estabelecido no projeto do equipamento.

A válvula de alívio é aquela que protege apenas o processo. Abre gradualmente em proporção ao aumento de pressão do sistema ao qual ela está instalada e após ser atingida a 
pressão de ajuste. Nessas válvulas, o curso de abertura é sempre proporcional à sob pressão do sistema.

Quando a pressão sobe bruscamente, a válvula de alívio não será suficiente para absorver esta variação. Em equipamentos, isso pode levar à destruição parcial ou explosões; em processos, perda da matéria-prima e de tempo. O acidente pode danificar também as instalações prediais da fábrica ou outros equipamentos próximos. Além disso, se ele ocorrer por falta do uso de válvula de segurança, a indústria será a única responsabilizada pelos prejuízos. Bolle et al. (1996) contribuem com informações detalhadas de escoamento bifásico através de válvulas de segurança.

Quando o esforço excede um valor predeterminado devido ao aumento de pressão na linha, no tanque ou no sistema a ser protegido, a mola cede permitindo a passagem do fluido. Deste modo, essa passagem se assemelha fisicamente ao comportamento de um bocal ou de um orifício. Local em que pode ocorrer o fenômeno da evaporação rápida

Existem simulações numéricas quando a válvula de segurança estoura e se originam ondas de choques, ver Rochette et al. (2007).

\subsubsection{Dispositivos de expansão}

Simões-Moreira e Bullard (2003) estudaram as ondas de evaporação em dois diferentes dispositivos de expansão: Em um tubo capilar e em uma válvula de expansão, como se mostra na Fig. 1.2. Em qualquer situação o líquido submete-se a um processo de expansão alcançando um estado metaestável e subitamente é formada uma onda de evaporação.

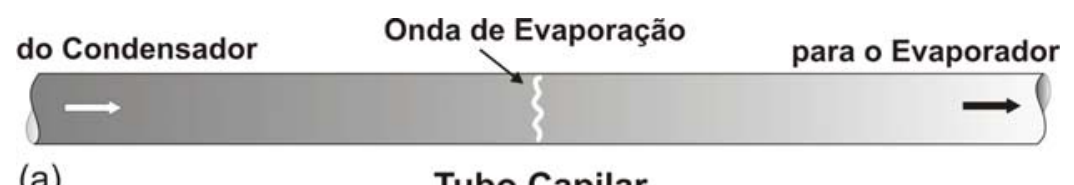

(a)

Tubo Capilar

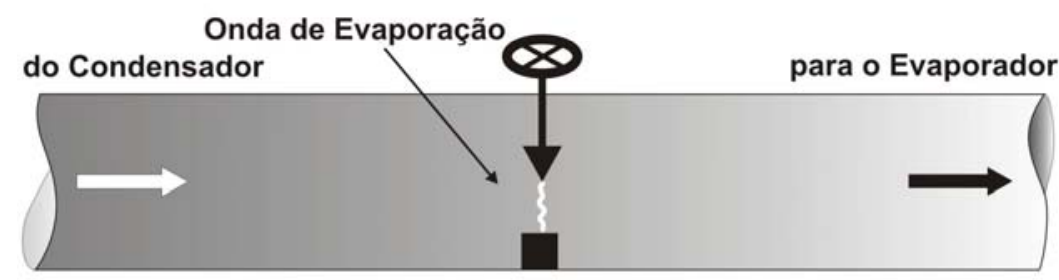

(b)

Válvula de Expansão

Figura 1.2 Ondas de evaporação (SIMÕES-MOREIRA; BULLARD, 2003), em: (a) Tubo capilar. (b) Válvula de expansão. 


\subsection{Descrição do Problema}

Quando um líquido está numa temperatura acima de seu ponto de ebulição, numa dada pressão, se diz que é superaquecido. Neste caso a fase líquida pode ter uma mudança súbita para a fase vapor, após perturbações. Em muitas aplicações a evaporação é causada por um superaquecimento local deste líquido a partir da transferência de calor com as paredes de uma estrutura de retenção. Entretanto, é possível conceber processos pelos quais, este fluido, pode sofrer uma evaporação adiabática, isto é, com transferência de calor insignificante com o meio. Por exemplo, a despressurização rápida ou “flashing” de um líquido saturado pode levar a um superaquecimento significativo e à produção súbita de diminutas bolhas de vapor que tendem a expandir. Dependendo do grau com que o fluido se afasta do equilíbrio, a evaporação pode ocorrer de maneira rápida ou até de forma explosiva.

Situações de “flashing” estão relacionadas com não-equilíbrio termodinâmico, isto é, quando a redução de pressão resulta neste superaquecimento do líquido, com a formação de bolhas de vapor. Como um exemplo, afastado do equilíbrio, o líquido tem sua pressão reduzida para valores abaixo de sua saturação, neste fenômeno as bolhas nucleadas, crescem a partir do calor extraído pelo líquido das vizinhanças, ou caso adiabático às custas da energia sensível restante no líquido. Devido à pressão do vapor do material ser relativamente grande, a combinação de um fluido dinâmico e instabilidades termodinâmicas conduzem à desintegração de gotas pequenas. O equilíbrio será alcançado quando a fração do líquido convertido ao vapor tenha extraído bastante energia do líquido residual.

Escoamento bifásico é de interesse em muitas áreas de ciência e engenharia. Nas áreas de segurança, uns dos tópicos é a liberação acidental de gases liquefeitos pressurizados tóxicos e inflamáveis. Phinasi (2005) afirma que na falha de um recipiente ou tubo, p.ex., como conseqüência de um diminuto buraco, resulta na formação de um jato bifásico contendo uma mistura de gotículas de líquido e vapor. Estas gotículas são geradas por uma ebulição súbita por sua temperatura estar acima do ponto de ebulição na pressão ambiente.

As primeiras pesquisas de ondas de choques estavam relacionadas com a teoria de escoamento de fluidos compressíveis, e logo depois, se estendeu à pesquisa experimental. Foi em 1965 que pela primeira vez que um tubo de dimensões pequenas foi aplicado como tubo de choque. A partir de então a pesquisa de onda de choque foi estendida para outras aplicações. A onda de choque é uma representação compreensiva do movimento de onda não linear, que ocorrem em sistemas nos quais a velocidade do fluido e a de propagação de 
informação ou velocidade do som, são comparáveis. Elas são caracterizadas por um salto abrupto e quase descontínuo que mudam nas características do meio. Através de uma onda de choque (compressão) há sempre aumento na pressão, temperatura e na densidade do escoamento. Entretanto, a diminuição destas propriedades pode ocorrer através de uma onda de choque de evaporação.

Neste trabalho, estudaremos o fenômeno da evaporação rápida em jato de fluido superaquecido ou metaestável. Este se inicia quando o jato em sua fase líquida, a alta temperatura e pressão, emerge de um diminuto bocal projetando-se numa câmara de baixa pressão, inferior à pressão de saturação. Durante o processo, ao cruzar-se a curva de saturação se observa que o fluido ainda permanece no estado de líquido superaquecido. Logo, subitamente o líquido superaquecido muda de fase por meio de uma onda de evaporação oblíqua, Simões-Moreira (2000). Esta mudança de fase transforma o líquido superaquecido numa mistura bifásica com alta velocidade distribuída em todas as direções com velocidades supersônicas cada vez maiores, expandindo-se até atingir a pressão a jusante, passando antes por uma onda de choque.

\subsection{Revisão Bibliográfica}

\subsubsection{Fenômenos evaporativos}

Hill (1991) estuda as ondas de choque de evaporação em líquidos superaquecidos usando uma instalação que permite uma rápida despressurização e que consiste em um tubo de vidro de teste situado abaixo de um reservatório grande e a baixa pressão. O objetivo deste estudo é aprender mais sobre os mecanismos físicos da ebulição explosiva (da qual uma onda de evaporação é um exemplo especifico), como também as propriedades que o escoamento produz. Foram usados fluidos de teste o refrigerante 12 e ou refrigerante 114 .

Simões-Moreira et al. (1993), Simões-Moreira (1994) e Simões-Moreira e Shepherd (1999) abordam o estudo de ondas de evaporação adiabática no dodecano $\left(C_{12} H_{26}\right)$. Por ser este fluido uma substância "retrograde” pode submeter-se a uma mudança de fase completa sob certas condições. Uso de aparatos de rápida descompressão num intervalo de temperaturas numa faixa de $180^{\circ} \mathrm{C}$ a $300^{\circ} \mathrm{C}$. Os experimentos consistiram numa súbita exposição do dodecano saturado em um ambiente de baixa pressão pela ruptura de um diafragma, ver 
Fig. 1.3. Acima de certo grau de aquecimento ondas de evaporação se propagam. Soluções numéricas demonstram que existe uma faixa de pressão e temperatura pela quais ondas de evaporação completa são observadas. Imagens fotográficas foram obtidas e estudadas, assim como mostra a Fig. 1.4.

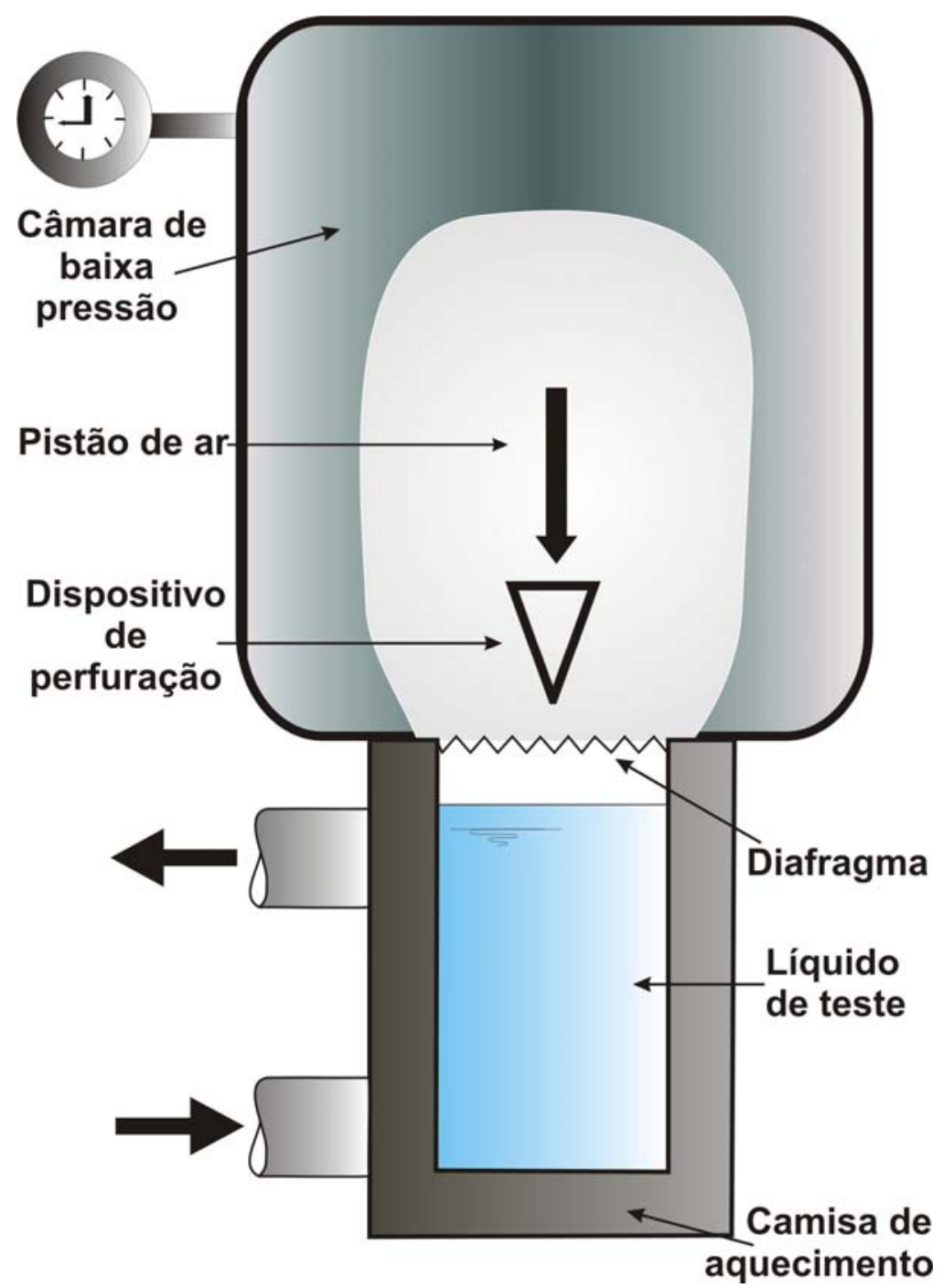

Figura 1.3 Arranjo experimental para observar ondas de evaporação (SIMÕES-MOREIRA et al.,1993). 


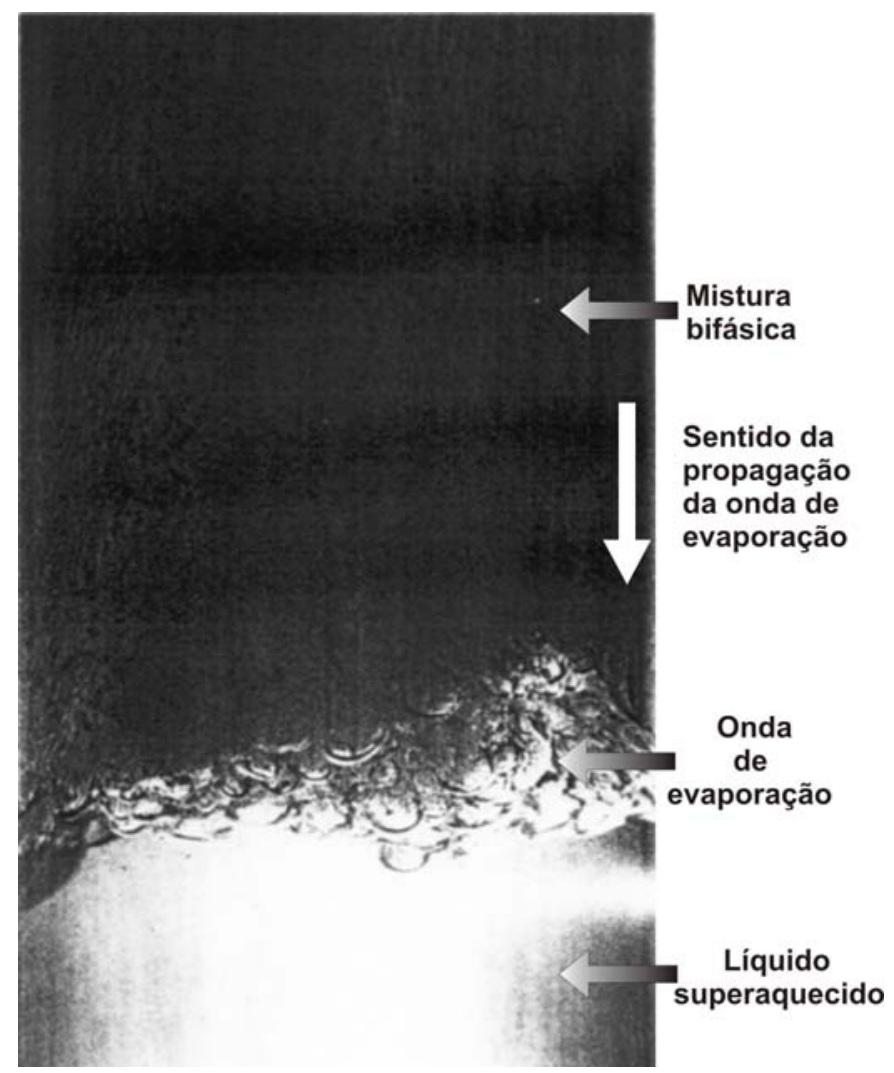

Figura 1.4 Fotografia de uma onda de evaporação em progresso do dodecano superaquecido no interior de um tubo de vidro. A temperatura é de $230^{\circ} \mathrm{C}$, a pressão acima da onda de evaporação é de $0.37 \mathrm{bar}$ e abaixo da onda é de $0.59 \mathrm{bar}$, e sua velocidade é de $47.2 \mathrm{~cm} / \mathrm{s}$ (SIMÕES-MOREIRA, 1994).

\subsubsection{Jatos de líquidos superaquecidos: Onda de choque}

Dentre os diferentes tipos de escoamentos, são de nosso interesse os de alto grau de metaestabilidade. Estes vão a formar parte dos jatos evaporativos altamente superaquecidos que encontram uma onda de choque durante a expansão bifásica. Em seguida, apresentaremos a linha de pesquisadores que contribuíram nesse estudo.

Kurschat et al. (1992) estuda a evaporação de jatos altamente superaquecidos de um bocal de expansão a uma câmara de vácuo. Usa um fluido de complexidade molecular elevada chamado perfluor-n-hexano $\left(C_{6} F_{14}\right)$. Desenvolveu um modelo para descrever os efeitos físicos básicos. Modelou a evaporação por uma onda de deflagração sônica seguido por uma expansão supersônica axissimétrica. Como no caso de jatos de gases supersônicos o estado final é atingido por uma onda de choque de compressão. Nos seus ensaios usou o parâmetro grau de superaquecimento $H=P_{\text {sat }}\left(T_{0}\right) / P_{\infty}$, onde $P_{\text {sat }}$ é a pressão de saturação. O bocal que usou tinha um perfil cônico convergente com diâmetro de saída 0.35 mm. A Fig. 1.5a mostra 
um jato de líquido subresfriado, ou seja, $H<1$ sem presença de mudança de fase. Com a diminuição da pressão da câmara, abaixo da pressão de saturação se dá inicio a pontos de nucleação heterogênea, de onde a evaporação se inicia (Fig. 1.5b).

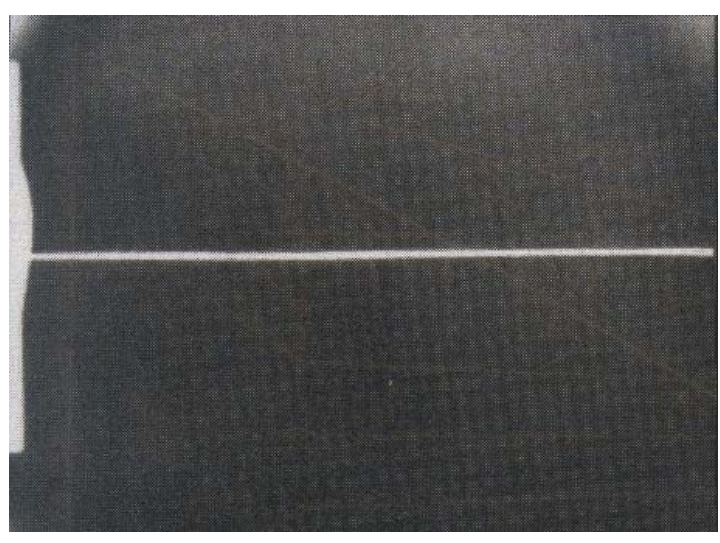

a) $H=0.5$

$T_{0}=20^{\circ} \mathrm{C}, P_{0}=2$ bar, $P_{\infty}=500$ mbar

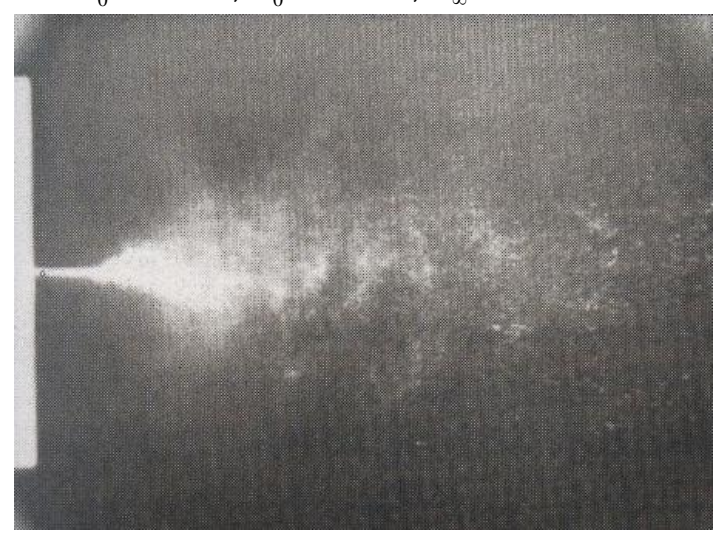

c) $H=6.8$

$T_{0}=20^{\circ} \mathrm{C}, P_{0}=2$ bar, $P_{\infty}=35$ mbar

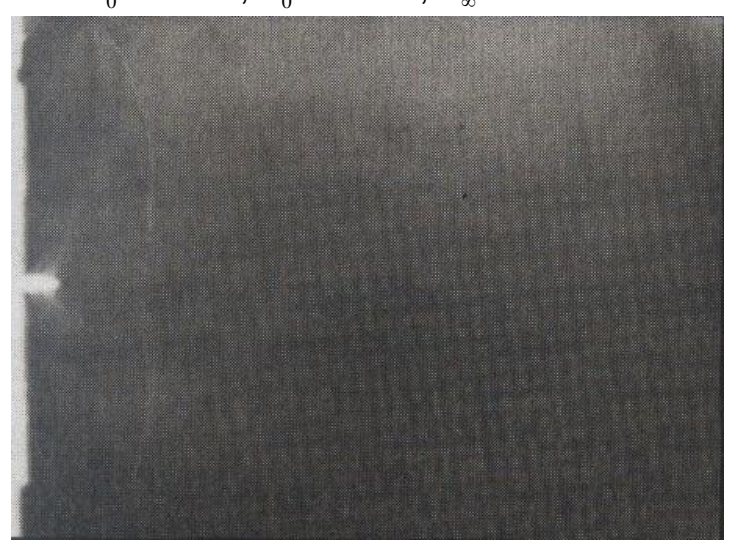

e) $H=600$

$T_{0}=120^{\circ} \mathrm{C}, P_{0}=15$ bar, $P_{\infty}=10 \mathrm{mbar}$

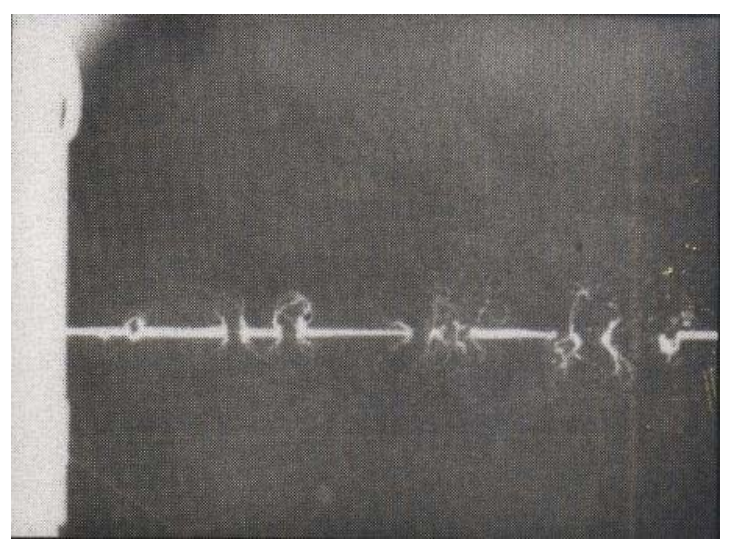

b) $H=2.4$

$T_{0}=20^{\circ} \mathrm{C}, P_{0}=2$ bar, $P_{\infty}=100$ mbar

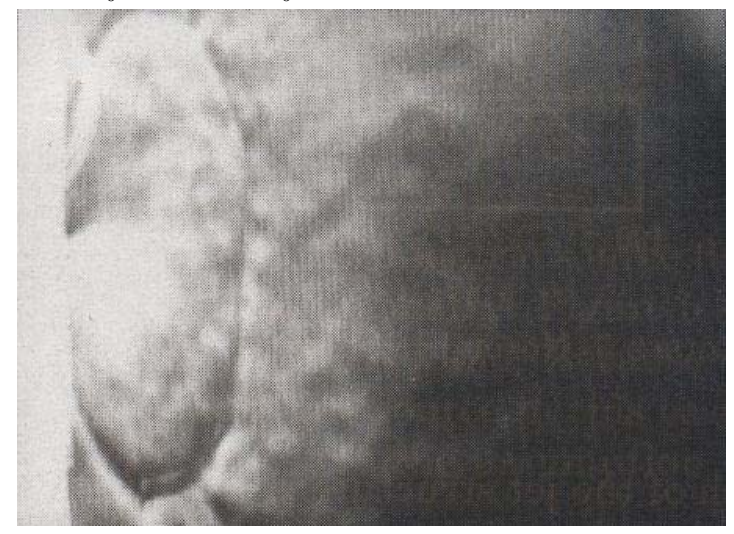

d) $H=370$

$T_{0}=100^{\circ} \mathrm{C}, P_{0}=15$ bar, $P_{\infty}=10 \mathrm{mbar}$

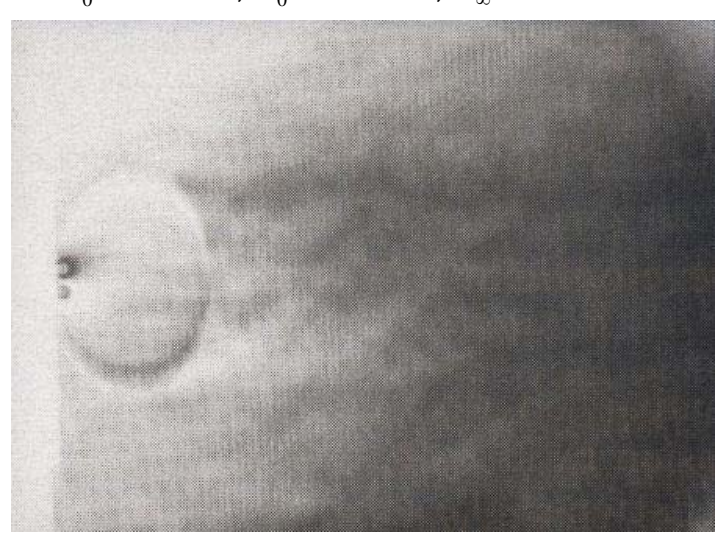

f) $P_{0} / P_{\infty}=1500$

$T_{0}=175^{\circ} \mathrm{C}, P_{0}=15$ bar, $P_{\infty}=10 \mathrm{mbar}$

Figura 1.5 Jato de diferentes geometrias e graus crescentes de superaquecimento $H$ e com fluido teste perfluor-n-hexano (KURSCHAT et al., 1992). 
Na Fig. 1.5c se diminui ainda mais a pressão da câmara obtendo-se uma mudança radical na estrutura do jato. Na Fig. 1.5d se visualiza um núcleo de líquido circundado por uma região supersônica terminada em um choque de formato elipsoidal. Já a Fig. 1.5e mostra o núcleo do líquido e a onda de choque. E finalmente no quadro da Fig. 1.5f mostra-se a evaporação, a onda de choque de formato esférico, dado a uma altíssima razão do grau de superaquecimento.

Athans (1995) realizou diversos experimentos com o fluido teste iso-octano, incluindo medições de vazão mássica, registros fotográficos, e também, comprimentos de extinção de jatos. No correspondente à geometria do jato, observa seu formato cônico. Com tudo, não foram apresentados algumas correlações empíricas ou analíticas para determinar estas dimensões em relação às condições experimentais. Na Fig. 1.6 observa-se a fotografia do jato a condições de estagnação $T_{0}=223^{\circ} \mathrm{C}$ e $P_{0}=2068 \mathrm{kPa}$, onde a parte mais clara representa o núcleo líquido e as linhas de corrente, representadas com setas brancas, se expandem pela mistura bifásica, seguindo a direção “quase perpendicular” ao eixo principal do jato até quase toda a extremidade final do jato. Também usa diversas técnicas fotográficas como "laser sheet”, "shadowngraph”, e iluminação direta.

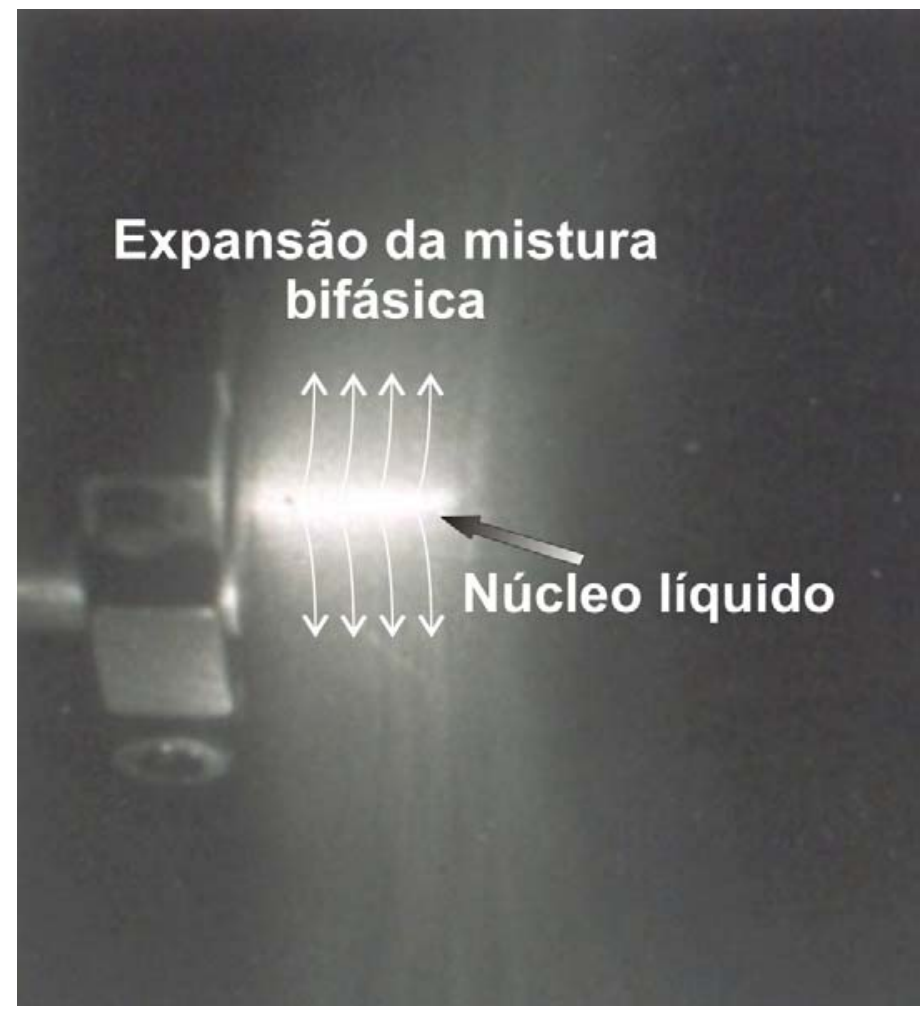

Figura 1.6 Imagem do jato de iso-octano com temperatura e pressão de injeção de $T_{0}=223^{\circ} \mathrm{C}$ e $P_{0}=2068 \mathrm{kPa}$, obtidas pelo método de "laser sheet" (ATHANS, 1995). 
Simões-Moreira (1999) aborda a teoria e aplicações das ondas de evaporação aos jatos evaporativos e generaliza a teoria das ondas evaporativas normais a ondas evaporativas obliquas. Também, estuda o jato evaporativo expandido em um bocal convergente isoentrópico que descarrega em uma câmara de pressão controlada. Analisa o problema da onda de choque que ocorrem em associação com os jatos evaporativos. Emprega uma abordagem 1D e elabora um código computacional chamado ShoWPhasT-1D, que fornece a solução à montante e à jusante de uma onda de evaporação. Finalmente compara seus resultados com os disponíveis em outras literaturas.

Vieira (1999) constrói um arranjo experimental para estudar o comportamento dos jatos evaporativos. Projeta um injetor para controlar e manter constantes a pressão e a temperatura de reservatório durante a injeção. Usa um bocal cônico convergente de secção reta com diâmetro de saída de 0,3 mm. Os fluidos testes que utilizou em seus experimentos são: o querosene e o n-dodecano $\left(C_{12} H_{26}\right)$. Para a visualização do escoamento usa o método de “Schlieren”. A analise dos jatos foi feita de forma qualitativa através de registros fotográficos e observa os seguintes tipos de jatos: contínuo, pulverizante, e evaporação na superfície. No caso de jatos com evaporação completa, constata a formação de ondas de choque com formatos elipsóides e esferóides considerando elevadas temperaturas. Por exemplo, jatos com uma pressão de injeção de $600 \mathrm{kPa}$ eram descarregados em uma câmara de baixa pressão, da ordem de 1,5 kPa. Quatro ensaios diferentes com diversas temperaturas de injeção foram testados, proporcionando um aumento sucessivo da pressão media de saturação do líquido. Estas seqüências das imagens são apresentadas na Fig. 1.7. Sendo que na primeira imagem a evaporação não é atingida, só observa-se uma linha reta do jato (Fig. 1.7a). Quando a temperatura continua aumentando-se se observa que a taxa de evaporação cresce abruptamente, diminuindo a proporção líquida do jato, deste modo a mudança de fase é atingida sob a superfície do núcleo líquido, e se expande formando uma onda de choque, como são mostradas nas imagens das Fig. 1.7b-d. 


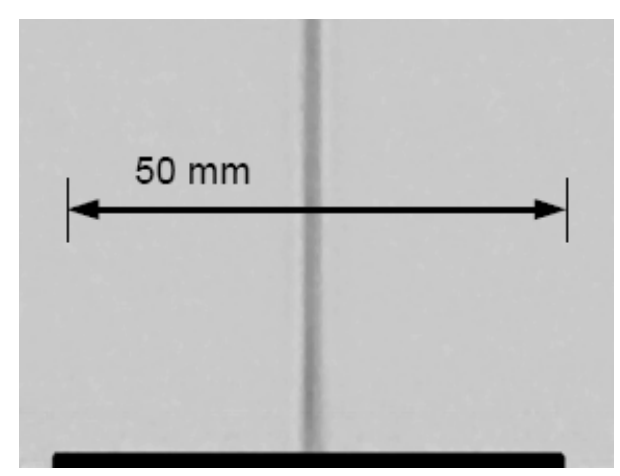

a)

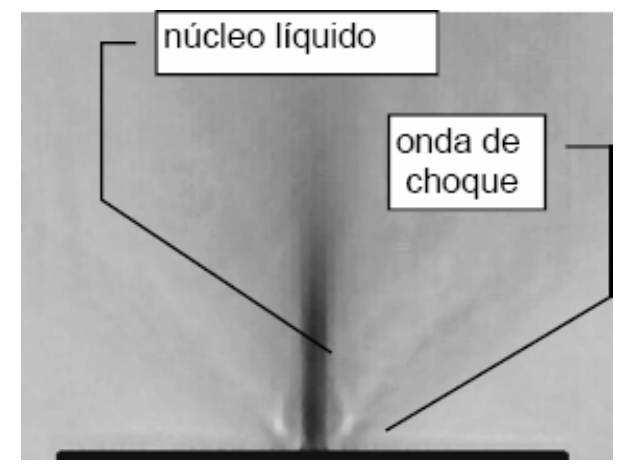

c)

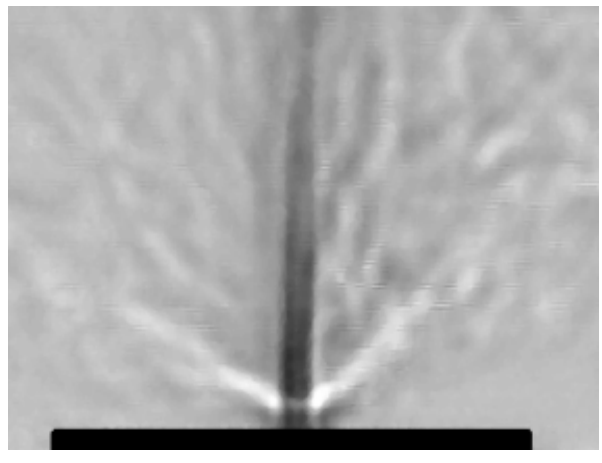

b)

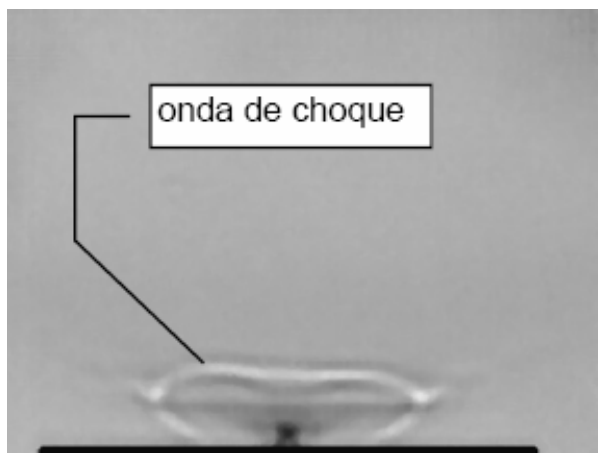

d)

Figura 1.7 Seqüência de imagens de jatos evaporativos de querosene com pressão de injeção de $600 \mathrm{kPa}$, e pressão da câmara igual a $1.6 \mathrm{kPa}$. A Temperatura de injeção é de 38, 95, 120 e $260^{\circ} \mathrm{C}$, respectivamente (VIEIRA, 1999).

Angelo (2000) apresentou uma modelagem matemática para estudar o fenômeno da dinâmica de evaporação dos jatos evaporativos. Adotou a hipótese de uma superfície de evaporação de formato cônico, afirmando que também apresenta bons resultados. Para a implementação do seu código usou o programa ShoWPhasT-1D (SIMÕES-MOREIRA, 1999). No que se refere à região de expansão bifásica afirmou que há grande variação das propriedades e um grande aumento no valor da velocidade, especialmente próximo à superfície de evaporação. Elaborou o código computacional de jatos evaporativos em uma região bidimensional axissimétrica: ShoWPhasT-2D.

Angelo (2004) estuda a expansão da mistura bifásica modelada em uma região 2D. As equações que governam o fenômeno são as equações de conservação da massa, conservação da quantidade de movimento, e conservação da energia, mais uma equação de estado para fluidos reais, p.ex., como é a equação de Lee-Kesler (veja Apêndice D). Este sistema de equações é estudado em sua forma conservativa e regime permanente. O método numérico que se usa para discretizar as equações de conservação estacionarias é o método de Diferenças 
Finitas com o esquema de MacCormack. Este método resolve a expansão bifásica, porém, não consegue achar a onda de choque diretamente. Um algoritmo de busca é implementado para capturar as ondas de choque. A implementação de um código foi feito para qualquer tipo de fluido, em particular, para substâncias de complexidade molecular elevada, Simões-Moreira (1997), porém, o iso-octano $\left(C_{8} H_{18}\right)$ é considerado como fluido teste. O código é chamado de: "Shock Waves With Phase Transition Two-Dimensional” (ShoWPhasT-2D) e por ser a primeira versão é também conhecido por ShoWPhasT-2D v1. Na Fig. 1.8 são apresentados a posição e o formato da onda de choque. Após a onda de choque a distribuição de cores não é valida, pois a solução não se estende além dela.
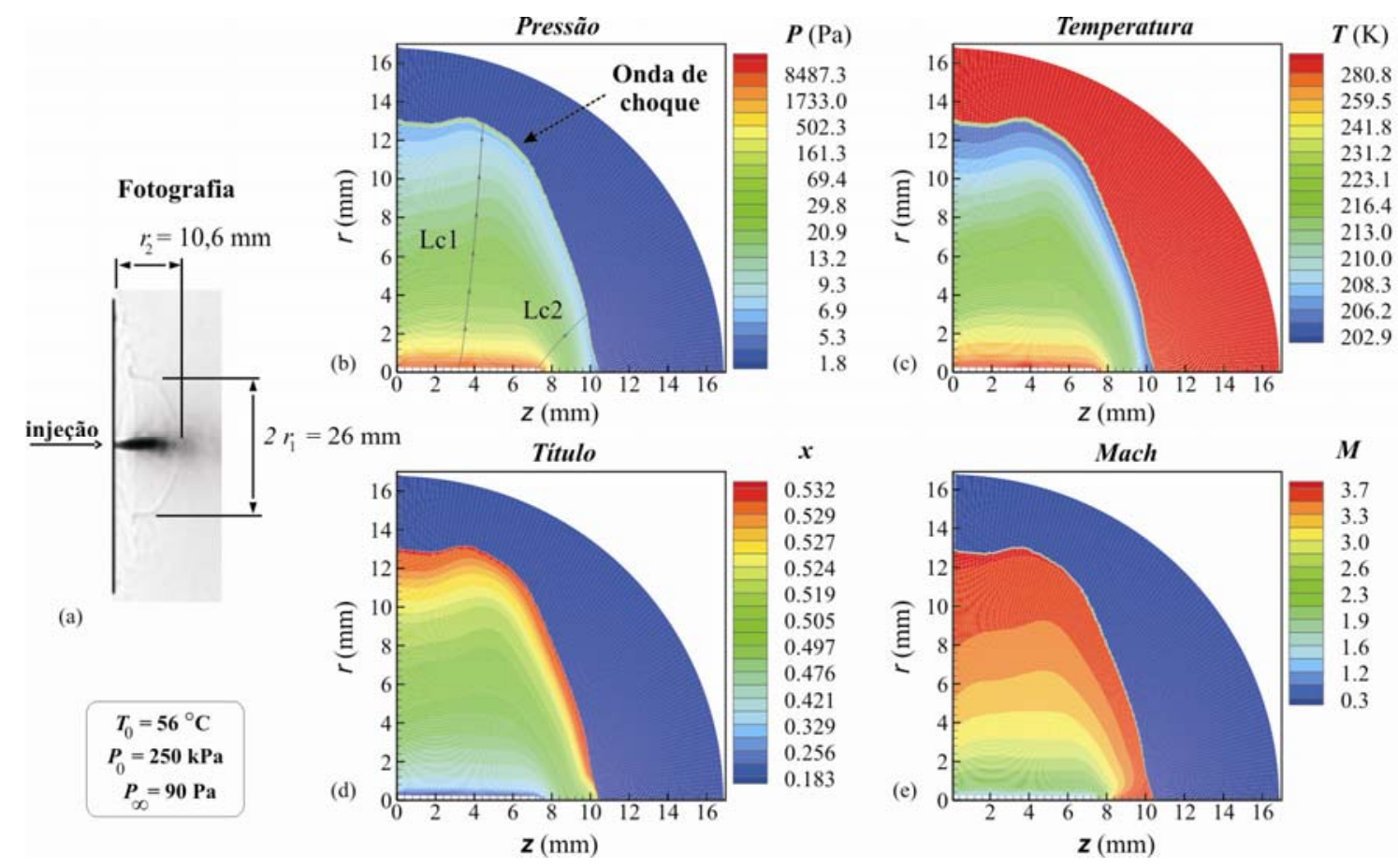

Figura 1.8 Resultados numéricos de Angelo (2004). Localização da Onda de Choque onde a pressão na câmara de injeção é de 90 Pa. (a) Fotografia de Vieira (2005). (b) Pressão. (c) Temperatura. (d) Título. (d) Número de Mach.

Vieira (2005) trabalhou experimentalmente no fenômeno de evaporação rápida de jatos de líquidos superaquecidos. Construiu um arranjo experimental para visualizar os jatos evaporativos com controle de pressão e temperatura de injeção constante. Dividiu-se basicamente em cinco sistemas (ver Fig. 1.9): (1) Sistemas de pressurização e de vácuo: Que controla e proporciona uma condição de pressão quase constante do ambiente onde o jato será expandido. (2) Sistema de aquecimento: Que aquece o líquido de teste até a temperatura 
desejada de injeção. (3) Sistema de injeção: Que injeta o líquido de teste a uma pressão constante de injeção e em um tempo determinado. (4) Sistema óptico - Método de “Schlieren": Este método permite o registro do gradiente de densidade em torno do jato além de ser empregado para visualizar ondas de choques, entre outros fenômenos, que podem estar presentes na evaporação de líquidos metaestáveis. (5) Sistema de aquisição de dados: formado por dois computadores, o primeiro computador gerencia todo o ensaio obtendo os dados de pressão e temperatura iniciais, posição da haste do injetor e sinais de saída para o disparo da injeção e da aquisição de imagens. O segundo computador é aquele que possui uma placa de aquisição de imagens, este recebe dois sinais a primeira para capturar as imagens do jato e a segunda para o acionamento da injeção. Com relação às ondas de choque, o mesmo enfatiza que não deve ser entendida simplesmente como normal ao escoamento, pois ela se projeta de forma mais complexa. Para observar o escoamento à jusante da onda de evaporação tomou, por exemplo, o ensaio $5435 \mathrm{~m} 10 c$, e forneceu as seguintes imagens. A Fig. 1.10a é a imagem original do ensaio. A Fig. 1.10b é uma imagem tratada por um filtro matemático do tipo subtrativo que permite um melhor contraste do fenômeno. Na verificação da variação de intensidade da imagem, em função do eixo horizontal tem características realçadas do jato, isto é, o núcleo do líquido e onda de choque (ver Fig. 1.10c). A Fig. 1.10.d é uma fotografia obtida pelo método de iluminação por detrás.

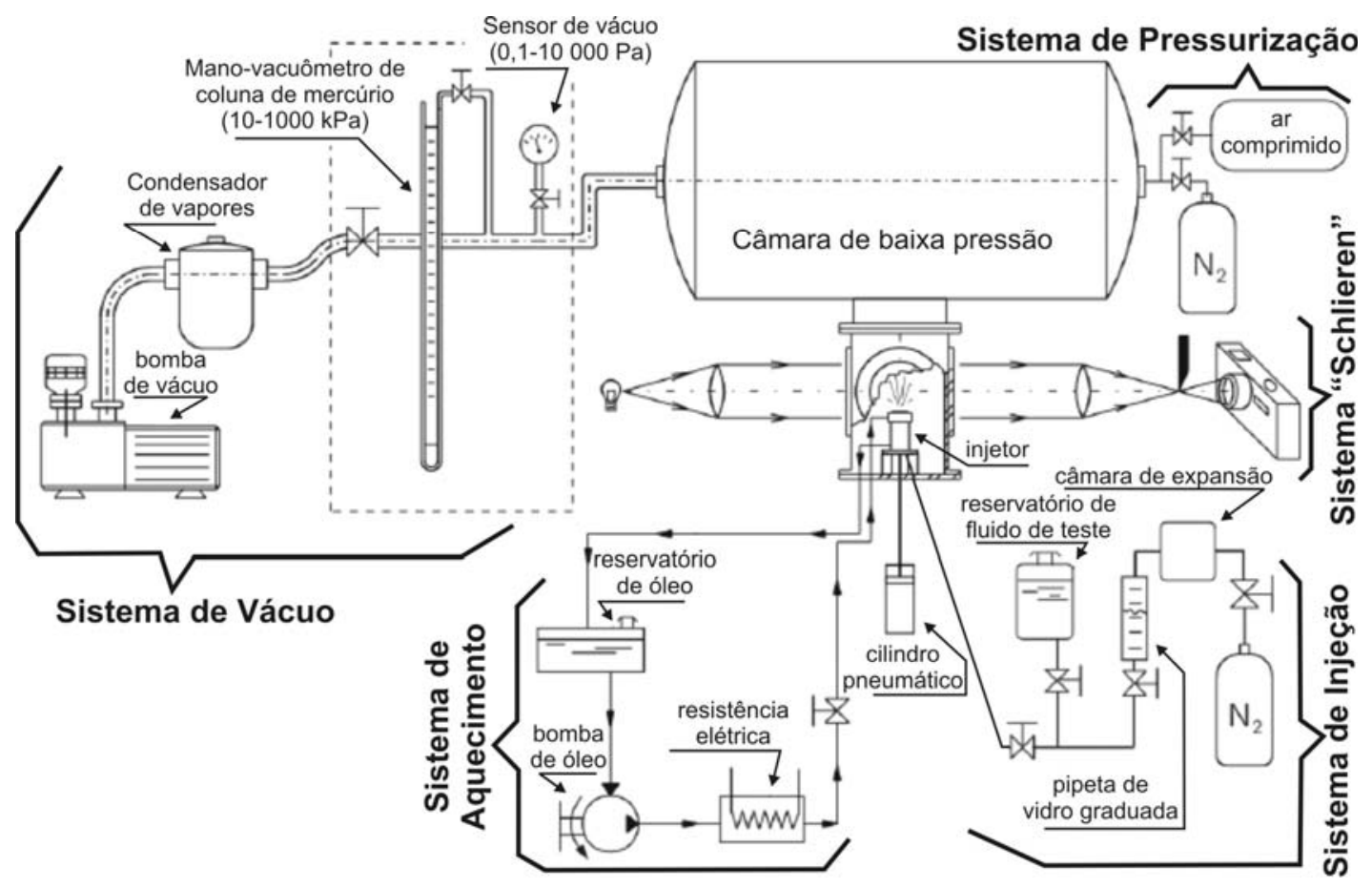

Figura 1.9 Esquema geral da bancada experimental, Vieira (2005). 


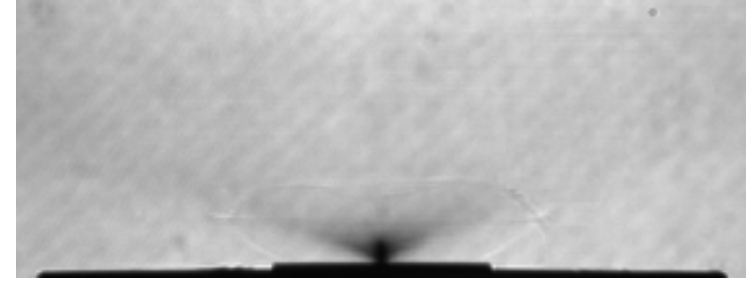

(a)

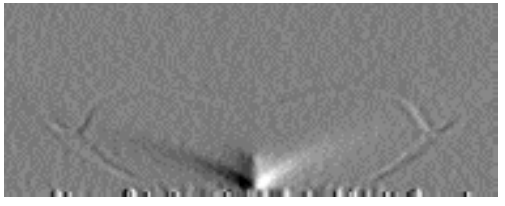

(c)

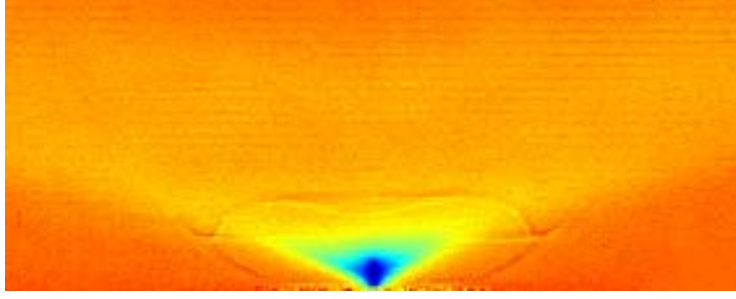

(b)

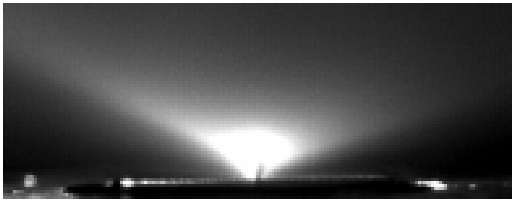

(d)

Figura 1.10 Ondas de choques (Ensaio 5435m10c; Vieira, 2005): (a) Imagem original do ensaio. (b) Imagem tratada com filtro matemático. (c) Imagem processada para destacar o núcleo do líquido e as ondas de choques. (d) Fotografia obtida com o método de iluminação por detrás.

\subsection{Objetivos}

Neste trabalho serão estudados os fenômenos associados à evaporação rápida (“flashing”) de jatos de líquidos superaquecidos, em geometria bidimensional. Este trabalho também estuda formas de se obter equação de estado mais acessível para simulações numéricas de misturas bifásicas tanto para os estados da pré-expansão bifásica quanto para os estados da expansão bifásica.

Os principais objetivos são:

- $\quad$ Revisão das técnicas de obtenção das propriedades termodinâmicas dos estados da préexpansão bifásica e do estado pós-onda de evaporação.

- Aperfeiçoamento da técnica de obtenção de equação de estado através de tabelas termodinâmicas, e aprofundando a análise dos fenômenos apresentados no trabalho feito por Angelo (2004). Os tópicos a serem tratados são referentes à parte computacional do software ShoWPhasT-2D v1, isto é:

$>$ Condições de contorno: Elaborar um tratamento especial para as condições de contorno ao longo da parede e na linha de simetria. 
> Qualidade da malha: Refinar a malha próxima à parede do domínio e próximo à linha inicial, após onda de evaporação.

$>$ Expansão bifásica: Substituir, no código, as rotinas de cálculo da Equação de Estado de Lee-Kesler por Tabelas termodinâmicas com rotinas de interpolação para acelerar o processo de avaliação das propriedades.

- $\quad$ Comparar os resultados numéricos obtidos com ensaios experimentais de Vieira (2005) e Vieira e Simões-Moreira (2007), e testes computacionais de Angelo (2004) e Angelo e Simões-Moreira (2007), realizados no Laboratório SISEA.

- $\quad$ Apresentar um novo método numérico, capaz de resolver conjuntamente o fenômeno da expansão bifásica e a captura da onda de choque, sem a necessidade de fazer uma busca de choque pós-processamento. Deste modo obtêm-se o campo de velocidades e termodinâmico de toda região de interesse, inclusive o título. Este método usará um esquema de “shockcapturing” com modelo do falso-transiente.

\subsection{Divisão da Tese}

O presente trabalho consta de oito capítulos incluindo os capítulos de Introdução e de Conclusões. São apresentados alguns algoritmos em pseudocódigo nos apêndices. O autor usou a linguagem de programação Fortran na implementação dos códigos computacionais.

O Capítulo 1 basicamente descreve o problema e indica os objetivos que se pretende alcançar. Apresenta-se a história da arte dos diferentes fenômenos de evaporação rápida, assim como também, informa-se de algumas aplicações preventivas e outras motivadoras.

O Capítulo 2 refere-se aos estados termodinâmicos da pré-expansão bifásica, e o estado termodinâmico pós-onda de evaporação. Neste capítulo, também, se abordam os conceitos de ângulo de onda e de giro, e as estruturas geométricas para obter o novo formato do núcleo líquido.

O Capítulo 3 corresponde à geometria do domínio físico e computacional, assim como também as condições de contorno. Este domínio físico é representado pelos estados da expansão bifásica. Finaliza este capítulo com a formulação conservativa das equações que governam os fenômenos da dinâmica dos gases, por exemplo, evaporação rápida de jatos de 
líquidos superaquecidos, em coordenadas cartesianas e nas coordenadas curvilíneas ortogonais.

O Capítulo 4 apresenta a solução numérica para o caso estacionário. É abordado o método de MacCormack, a viscosidade artificial, decodificação das variáveis e a varredura para capturar a onda de choque. Alem disso, se constrói um método de refinamento para a malha.

O Capítulo 5 apresenta a solução numérica para o caso não estacionário. É abordado o esquema “Dispersion-Controlled Dissipative” (DCD).

O Capítulo 6 compara os resultados numéricos com os resultados experimentais. Um grupo de dados numéricos é obtido, variando a pressão da câmara injeção ou as condições de reservatório.

No Capítulo 7 apresentamos, soluções numéricas para os campos de velocidades e termodinâmico usando o código computacional DCD-2D v1 (Dispersion-Controlled Dissipative - Two-Dimensional, version 1). Obtemos resultados para o caso do modelo de falso-transiente.

O Capítulo 8 apresenta-se as conclusões e continuidade do trabalho. 


\section{ESTADOS TERMODINÂMICOS DA PRÉ-EXPANSÃO BIFÁSICA}

O fenômeno de evaporação rápida em jatos evaporativos de líquidos altamente superaquecidos, passa por seis estados termodinâmicos (Fig.2.2), os quais, para um melhor estudo, se dividem em dois grupos: estados termodinâmicos da pré-expansão bifásica e estados termodinâmicos da expansão bifásica.

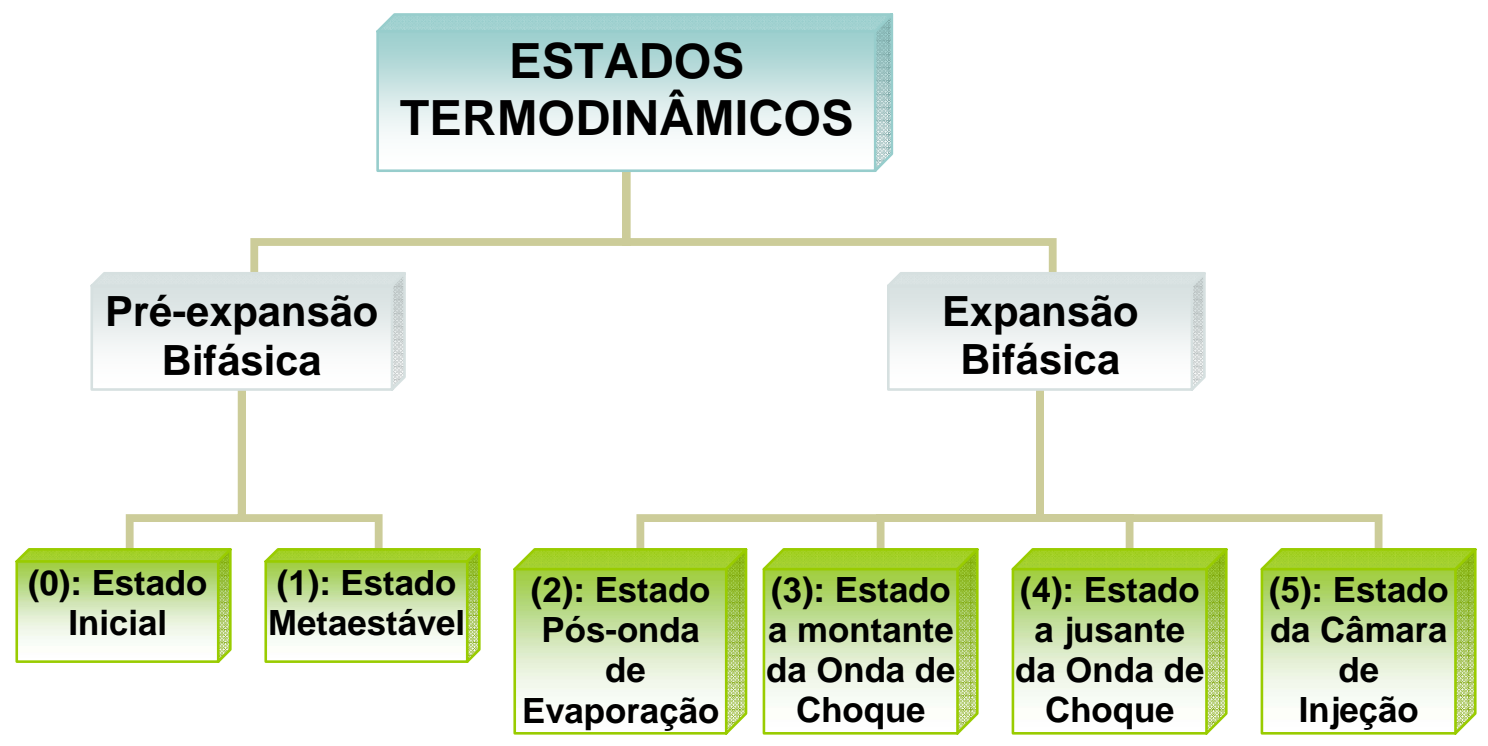

Figura 2.1 Organograma da divisão dos Estados Termodinâmicos que envolvem o fenômeno.

Os estados termodinâmicos que correspondem ao grupo da pré-expansão bifásica são dois, a saber: estado inicial (ou de estagnação ou de reservatório), denotado por (0) e estado metaestável, denotado por (1). Os estados termodinâmicos que correspondem ao grupo da expansão bifásica são quatro, a saber: estado pós-onda de choque de evaporação, denotado por (2), estado à montante da onda de choque de compressão, denotado por (3), estado à jusante desta onda de choque, denotado por (4) e estado da câmara de injeção, denotado por (5). Na Fig. 2.1 apresentamos uma representação simplificada do processo termodinâmico, enquanto na Fig. 2.2 uma representação esquemática dos estados que envolvem o fenômeno.

Os processos envolvidos na pré-expansão bifásica são: 
$(0) \rightarrow(1)$ : processo isotérmico metaestável.

$(1) \rightarrow(2)$ : processo da onda de evaporação.

Os processos envolvidos na expansão bifásica são:

$(2) \rightarrow(3)$ : processo da expansão bifásica.

(3) $\rightarrow(4)$ : processo da onda de choque.

(4) $\rightarrow(5)$ : processo do ajustes de pressões.

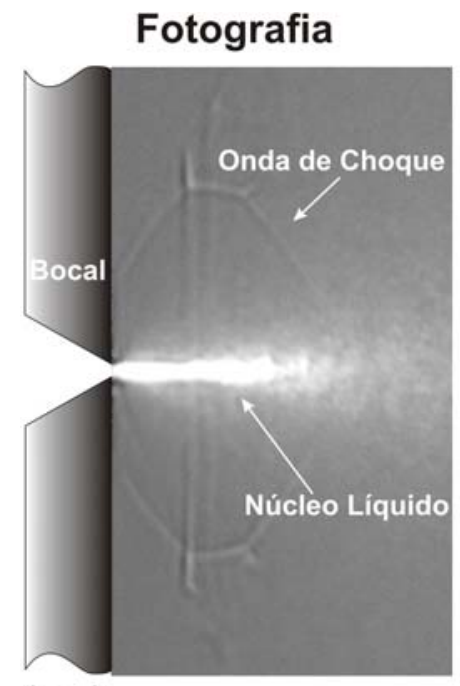

( a )

\section{Representação Esquemática}

(0)

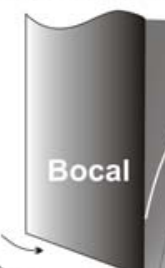

Onda de Choque

(3) $(4)$

(4)

(2)

(1)

(5)

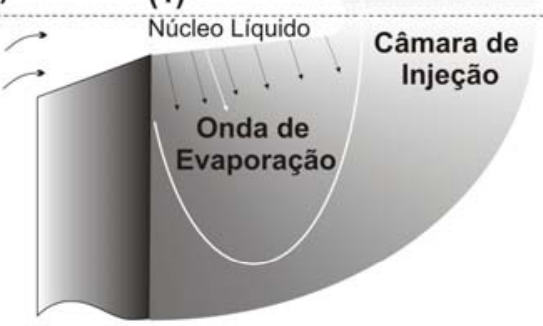

( b )

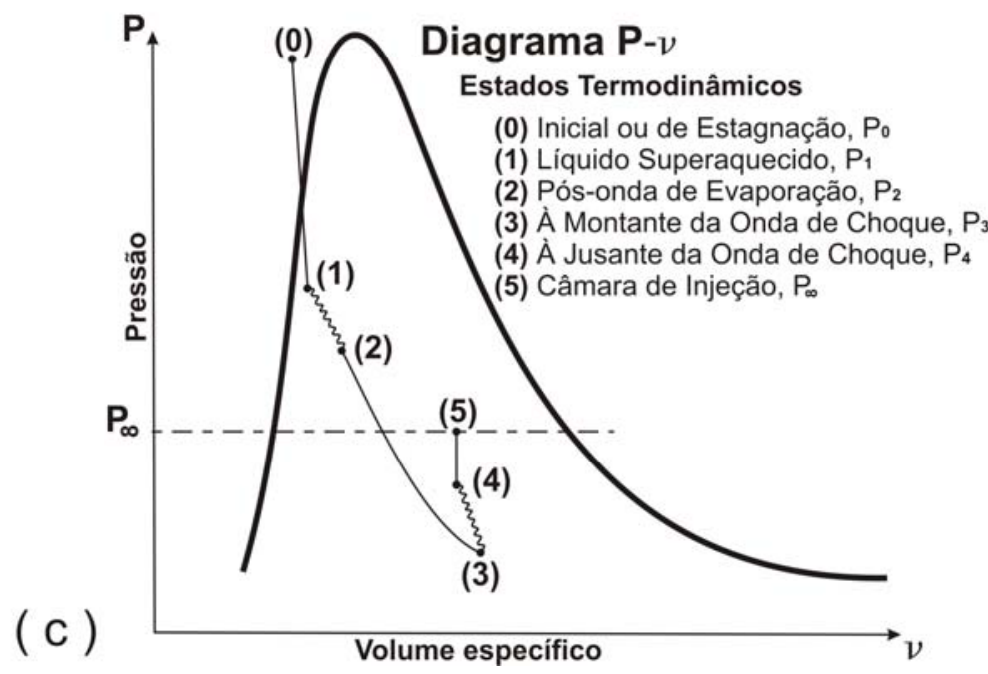

Figura 2.2 Representação dos estados termodinâmicos nos jatos evaporativos altamente superaquecidos. (a) Fotografia de Vieira (2005). (b) Representação esquemática do fenômeno. (c) Comportamento termodinâmico do fenômeno no diagrama $P-v$. 
Neste capítulo abordaremos os estados termodinâmicos da pré-expansão bifásica e o estado pós-onda de evaporação. É importante realçar que, para obtenção destes estados, precisamos conhecer quatro dados importantes, que são: raio do bocal, vazão em massa, coeficiente de descarga e comprimento do núcleo líquido. Estes dados foram determinados experimentalmente, por Vieira (2005). Uma vez conseguidas as propriedades termodinâmicas destes estados, é preciso conhecer alguns outros parâmetros geométricos, que são: ângulo de onda e ângulo de giro, que nos dão mais informação do vetor velocidade. E por último redesenharemos o formato cônico do núcleo líquido para um novo de formato paraboloidal, com isto suavizamos as variações das propriedades, isto significa que obteremos novos valores (valores corrigidos) para o raio do bocal e para o comprimento do núcleo líquido.

\subsection{Estado Inicial}

Sabemos que a velocidade e as propriedades termodinâmicas podem variar à medida que o escoamento flui. De tal modo que precisamos conhecer condições de referência que podem ser utilizadas para relacionar condições de um estado a outro num determinado processo. Para qualquer escoamento, a condição de referência é obtida quando o fluido é levado ao repouso, isto é: $V_{0}=0$. Chamaremos este estado de inicial (ou de reservatório), o qual, denotaremos simbolicamente por $(0)$ e suas propriedades termodinâmicas por $\left\{P_{0}, T_{0}, v_{0}, h_{0}, s_{0}\right\}$ para referirmos à pressão, temperatura, volume específico, entalpia específica e entropia específica, respectivamente.

É importante frisar que as condições iniciais para a pressão e a temperatura são muitos altos num reservatório. As duas únicas propriedades termodinâmicas de injeção no estado (0) são: $\left\{P_{0}, T_{0}\right\}$, e foram escolhidos os mesmos valores iniciais de pressão e temperatura fornecidos por Vieira (2005).

Os jatos evaporativos altamente superaquecidos foram originados pela injeção de líquidos mantidos à alta temperatura e pressão em câmaras de baixa pressão (abaixo da pressão de saturação à temperatura de injeção). Vieira (2005), em seus experimentos usa bocais do tipo cônico-convergente feito de aço-carbono, com o perfil interno do canal usinado por eletroerosão. O bocal de nosso interesse tem o diâmetro maior (entrada) de 3,0 [mm], diâmetro menor (saída) de $0,3[\mathrm{~mm}]$ e vértice de $20^{\circ}$. O estado inicial está localizado na entrada deste bocal convergente, como esquematicamente se mostra na Fig. 2.2b. 


\subsection{Estado Metaestável}

O estado metaestável, denotado por (1), está identificado na saída do bocal e o interior do núcleo líquido. O processo envolvido para a obtenção do estado metaestável, se chama processo isotérmico metaestável e é denotado por: $(0) \rightarrow(1)$. O estado (1) será estabelecido quando conhecermos duas propriedades termodinâmicas, a saber, pressão metaestável e volume específico metaestável. Em seguida, ofereceremos os procedimentos para o cálculo da pressão e volume específico, metaestáveis. Nesta seção, utilizaremos três dados experimentais fornecidos por Vieira (2005): raio do bocal, vazão mássica e o coeficiente de descarga.

\subsubsection{Pressão metaestável}

Para o cálculo da pressão metaestável, denotada por $P_{1}$, utilizaremos a equação de Bernoulli deduzida da equação de Euler no sistema de coordenadas cartesianas $(x, y, z)$. Denota-se a aceleração da gravidade local pelo vetor $\vec{g}=\left(g_{x}, g_{y}, g_{z}\right)$, a pressão por $P$, a densidade por $\rho$, a velocidade por $V$ e o deslocamento na direção do "eixo z" por $z$. Então a equação de Bernoulli, para um escoamento em regime permanente, ao longo de uma linha de corrente e sem atrito é dada por:

$$
\int \frac{d P}{\rho}+\frac{V^{2}}{2}+z g_{z}=c t e
$$

e para um escoamento incompressível temos:

$$
\frac{P}{\rho}+\frac{V^{2}}{2}+z g_{z}=c t e
$$

Agora queremos aplicar a Eq. (2.2) em dois pontos diferentes dados pelos estados (0) e (1). Pela hipótese do escoamento ser incompressível, temos que a densidade nos estados (0) e (1) são iguais, isto é, $\rho_{0}=\rho_{1}$. Então temos:

$$
\frac{P_{0}}{\rho_{1}}+\frac{V_{0}^{2}}{2}+z_{0} g_{z}=\frac{P_{1}}{\rho_{1}}+\frac{V_{1}^{2}}{2}+z_{1} g_{z}
$$


Por outro lado tem-se deslocamento do escoamento numa única direção, isto é, $z_{0}=z_{1}$, porém, Vieira (2005) usa uma aproximação, isto é: $z_{0} \cong z_{1}$. Por último usaremos a condição de estagnação $V_{0}=0$, assim temos:

$$
\frac{P_{0}}{\rho_{1}}=\frac{P_{1}}{\rho_{1}}+\frac{V_{1}^{2}}{2}
$$

A vazão mássica "teórica" é definida por:

$$
\dot{m}_{t}=\rho_{1} A_{b} V_{1}
$$

onde $A_{b}$ é a área da saída do bocal convergente.

A partir dos ensaios experimentais (Vieira, 2005) são obtidos o coeficiente de descarga $C_{D}$ e a vazão mássica "real" $\dot{m}$. Assim, a vazão em massa "real" que escoa através do bocal é o produto do coeficiente de descarga com a vazão em massa "teórica":

$$
\dot{m}=\dot{m}_{t} C_{D}
$$

A partir das Eqs. (2.5) e (2.6) isolamos a velocidade e substituímos na Eq. (2.4), temos a pressão metaestável:

$$
P_{1}=P_{0}-\frac{\dot{m}^{2}}{2 \rho_{1} A_{b}^{2} C_{D}^{2}}
$$

Observe-se que na Eq. (2.7) para calcular o valor da pressão metaestável precisamos conhecer o valor da densidade, $\rho_{1}$.

\subsubsection{Volume específico metaestável}

O volume específico metaestável será denotado por $v_{1}$. Em muitos casos a aproximação do volume específico, é dada pelo volume especifico do líquido saturado, para uma determinada isotérmica. Assim:

$$
v_{1}=v_{L}\left(T_{0}\right)
$$


O valor de $v_{1}$ dado em Eq. (2.8) foi inicialmente considerado para nossos cálculos, mas, para melhorar a precisão deste valor, foram feitas algumas aproximações diferenciais. Considerese a definição da diferencial total de $v$ dado por:

$$
d v=\left.\frac{\partial v}{\partial P}\right|_{s} d P+\left.\frac{\partial v}{\partial s}\right|_{P} d s
$$

Logo, assumindo a hipótese de estar num processo isoentrópico $(d s=0)$, integrando e avaliando os incrementos de $v_{1}$ e de $P_{1}$ na saturação, tem-se a seguinte relação termodinâmica:

$$
v_{1}=v_{\sigma}+\left.\left(P_{1}-P_{\sigma}\right) \frac{\partial v}{\partial P}\right|_{s}
$$

Onde:

$$
\left.\frac{\partial v}{\partial P}\right|_{s}=\frac{v_{1}-v_{0}}{P_{1}-P_{0}}
$$

Como se pode notar não é possível calcular diretamente o valor de $v_{1}$, pois não conhecemos diretamente o valor de $P_{1}$ a não ser o dado pela Eq. (2.7). Por isso é necessário fazer uma aproximação numérica que atualize os valores de $v_{1}$ até sua convergência. Na Fig. A.1 do Apêndice A, descrevemos um algoritmo em pseudocódigo para o cálculo de $P_{1}$ e $v_{1}$. Neste algoritmo são envolvidas as Eqs. (2.7), (2.8), (2.10) e (2.11).

\subsection{Estado Pós-onda de Evaporação}

O estado pós-onda de evaporação, denotado simbolicamente por (2), é aquele obtido como resultado do processo termodinâmico da onda de evaporação, $(1) \rightarrow(2)$. Este estado é bem característico porque encontra uma onda de evaporação (conhecida na área de combustão por onda de deflagração), onde as propriedades termodinâmicas pós-onda de evaporação são obtidas pela solução de Chapman-Jouguet.

Deduziremos as equações gerais de conservação que nos ajudam a obter a solução à jusante desta onda de evaporação, para tal efeito utilizaremos o conceito de descontinuidade 
para representar uma onda de evaporação ou uma onda de choque, mas não sem antes considerar algumas hipóteses simplificadoras e algumas notações para logo poder estudar as equações de salto.

\subsubsection{Notações e hipóteses simplificadoras}

Para se entender melhor estas notações e simplificações observem a Fig. 2.3.

\section{- $\quad$ Notações}

a) Se $f$ denota qualquer propriedade de um fluido. Então $f_{1}, f_{2}$ denotam as propriedades no estado (1) e (2), respectivamente. Defina-se a notação compacta de $f$ por:

$$
[f]=\left(f_{2}-f_{1}\right)
$$

Além disso, se ambos estados coincidem denotaremos por:

$$
f=f_{1}=f_{2}
$$

b) O espaço Euclidiano 3D (Tridimensional) no sistema de coordenadas cartesianas será $(x, y, z)$.

c) Um volume de controle arbitrário será denotado por $V$ e sua superfície de controle por $S$.

d) O vetor normal exterior à superfície $S$, denotado por $\vec{n}$, é unitário $\left(|\vec{n}|^{2} \equiv n^{2}=1\right)$ e nos estados (1) e (2) satisfazem:

$$
\vec{n}=\vec{n}_{1}=-\vec{n}_{2}
$$

e) Definamos o vetor velocidade por $\vec{V}=\left(V_{1}, V_{2}, V_{3}\right)$ e seu módulo ao quadrado por

$$
|\vec{V}|^{2} \equiv V^{2}=V_{1}^{2}+V_{2}^{2}+V_{3}^{2}
$$

f) A componente do vetor velocidade, na direção da normal exterior à descontinuidade, será denotado por: 


$$
W_{i}=\vec{V}_{i} \cdot \vec{n}, \quad i=1,2
$$

g) A componente do vetor velocidade, na direção tangencial à descontinuidade, será denotada por:

$$
\mathrm{U}_{i}=\vec{V}_{i} \cdot \vec{t}, \quad i=1,2
$$

e satisfaz:

$$
\mathrm{U}_{1}=\mathrm{U}_{2}
$$

h) O módulo do vetor velocidade nos estados (1) e (2) será denotado por:

$$
V_{i}^{2}=W_{i}^{2}+\mathrm{U}_{i}^{2}, \quad i=1,2
$$

i) A decomposição da área superficial para os estados (1) e (2) satisfaz:

$$
A=A_{1} \cup A_{2}
$$

j) A energia total é definida por:

$$
E=\rho e+\frac{1}{2} \rho V^{2}
$$

k) A entalpia especifica é definida por:

$$
h=e+\frac{P}{\rho}
$$

1) A entalpia de estagnação é definida por:

$$
h_{0}=h+\frac{1}{2} V^{2}
$$

m) Denote-se a vazão mássica por unidade de área por $J$, e define-se como:

$$
J_{i}=\rho_{i} W_{i}, \quad i=1,2
$$

n) Define-se o título como a fração mássica de vapor contida em uma mistura, e denote-se por $x$. Se $f$ denota outras propriedades termodinâmicas, pode se expressar numa região de mistura bifásica através da lei elementar de mistura: 


$$
f=(1-x) f_{L}+x f_{V}
$$

o) Para o uso da tabela de propriedades termodinâmicas da substância do iso-octano, utilizaremos a sub-rotina: Interpolação_Lee-Kesler (veja como usar na Tabela D.5 do Apêndice D).

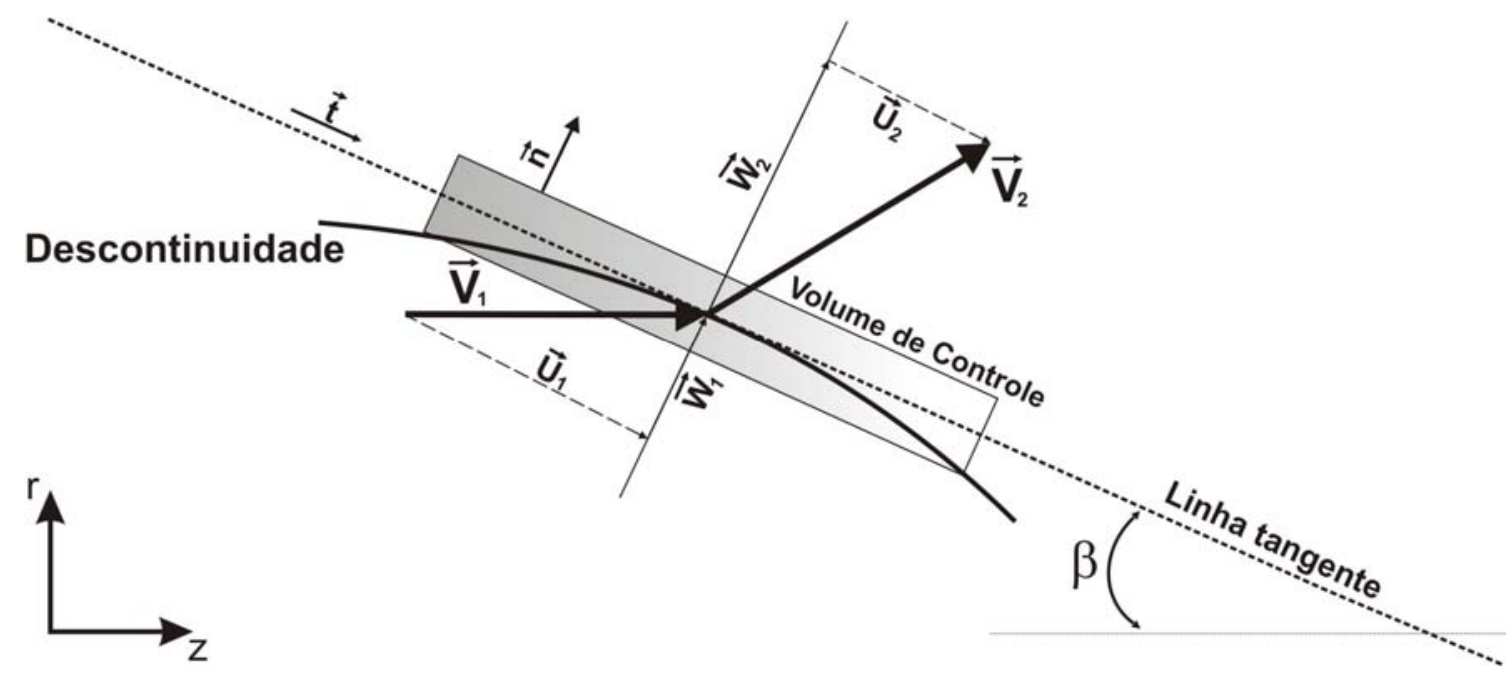

Figura 2.3 Onda de descontinuidade em um volume de controle e propriedades à montante e à jusante da descontinuidade.

- $\quad$ Hipóteses simplificadoras

a) O escoamento em estudo é 1D (Unidimensional), na direção horizontal do eixo “ $x$ ”, estacionário e não-viscoso (invíscido), isto significa, que as forças devido à viscosidade são desprezíveis.

b) As áreas transversais de um volume de controle contendo uma descontinuidade são iguais, isto é:

$$
A_{1}=A_{2}
$$

c) As velocidades e as propriedades termodinâmicas nos estados (1) e (2) são constantes e uniformes. Isto implica que, por exemplo, a velocidade e a densidade não variam em cada ponto de suas respectivas áreas. 
p) Se $\vec{f}$ representa a força de campo por unidade de massa, então, esta é nula.

j) O tensor dos esforços viscosos, $\tau$ (tensor de ordem 3), é nulo para o caso de fluidos invíscidos.

\subsubsection{Equações de salto}

As equações de salto são formadas pela equação de conservação da massa, conservação da quantidade de movimento e conservação da energia. Neste estudo, consideraremos as equações integrais definidas num volume de controle tal como se mostra na Fig. 2.3.

\section{- Conservação da massa}

A equação de conservação da massa na sua forma integral é dada por:

$$
\frac{\partial}{\partial t} \int_{V} \rho \mathrm{d} V+\int_{S} \rho \vec{V} \cdot \vec{n} \mathrm{~d} S=0
$$

e para um regime permanente temos:

$$
\int_{S} \rho \vec{V} \cdot \vec{n} \mathrm{~d} S=0
$$

Pela definição de integral de superfície e pela Eq. (2.20), temos:

$$
\int_{A_{1}} \rho_{1} \vec{V}_{1} \cdot \vec{n}_{1} \mathrm{dA}+\int_{A_{2}} \rho_{2} \vec{V}_{2} \cdot \vec{n}_{2} \mathrm{dA}=0
$$

Como os integrandos são constantes, temos:

$$
\rho_{1}\left(\vec{V}_{1} \cdot \vec{n}\right) A_{1}=\rho_{2}\left(\vec{V}_{2} \cdot \vec{n}\right) A_{2}
$$

Usando Eq. (2.26) em Eq. (2.30), temos:

$$
\rho_{1} W_{1}=\rho_{2} W_{2}
$$

Pela notação dada em Eq. (2.24), temos:

$$
J_{1}=J_{2}
$$


Esta é a equação da conservação da massa para o volume de controle que contem a descontinuidade.

\section{- Conservação da quantidade de movimento}

A equação de conservação da quantidade de movimento na sua forma integral é dada por:

$$
\frac{\partial}{\partial t} \int_{V} \rho \vec{V} \mathrm{~d} V+\int_{S} \rho \vec{V} \vec{V} \cdot \vec{n} \mathrm{~d} S=\int_{V} \rho \vec{f} \mathrm{~d} V-\int_{S} P \vec{n} \mathrm{~d} S+\int_{S} \tau \cdot \vec{n} \mathrm{~d} S
$$

Dado que o fluido é estacionário e invíscido, temos:

$$
\int_{\mathrm{S}} \rho \vec{V} \vec{V} \cdot \vec{n} \mathrm{dS}=\int_{\mathrm{V}} \rho \vec{f} \mathrm{dV}-\int_{\mathrm{S}} P \vec{n} \mathrm{dS}
$$

e como as forças de campo são desprezíveis, temos:

$$
\int_{S}(\rho \vec{V} \vec{V} \cdot \vec{n}+P \vec{n}) \mathrm{d} S=0
$$

Pela definição de integral de superfície e pela Eq. (2.20), temos:

$$
\int_{A_{1}}\left(\rho_{1} \vec{V}_{1} \vec{V}_{1} \cdot \vec{n}_{1}+P \vec{n}_{1}\right) \mathrm{dA}+\int_{A_{2}}\left(\rho_{2} \vec{V}_{2} \vec{V}_{2} \cdot \vec{n}_{2}+P \vec{n}_{2}\right) \mathrm{dA}=0
$$

é direto se notar que os integrandos de ambas integrais são constantes, logo:

$$
\left[\rho_{1} \vec{V}_{1}\left(\vec{V}_{1} \cdot \vec{n}\right)+P_{1} \vec{n}\right] A_{1}=\left[\rho_{2} \vec{V}_{2}\left(\vec{V}_{2} \cdot \vec{n}\right)+P_{2} \vec{n}\right] A_{2}
$$

Fazendo uso da Eq. (2.26) e multiplicando escalarmente por $\vec{n}$, temos:

$$
\rho_{1}\left(\vec{V}_{1} \cdot \vec{n}\right)\left(\vec{V}_{1} \cdot \vec{n}\right)+P_{1} \vec{n} \cdot \vec{n}=\rho_{2}\left(\vec{V}_{2} \cdot \vec{n}\right)\left(\vec{V}_{2} \cdot \vec{n}\right)+P_{2} \vec{n} \cdot \vec{n}
$$

sabendo que, o vetor normal $\vec{n}$ é unitário e usando a Eq. (2.16), temos:

$$
P_{1}+\rho_{1} W_{1}^{2}=P_{2}+\rho_{2} W_{2}^{2}
$$

Usando a notação dada em Eq. (2.24), temos:

$$
P_{1}+J_{1} W_{1}=P_{2}+J_{2} W_{2}
$$


E, portanto, temos a equação de conservação da quantidade de movimento.

\section{- Conservação da energia}

A equação de conservação da energia em sua forma integral é dada por:

$$
\frac{\partial}{\partial t} \int_{V} E \mathrm{~d} V+\int_{S} E \vec{V} \cdot \vec{n} \mathrm{~d} S=\int_{V} \rho \vec{f} \cdot \vec{V} \mathrm{~d} V+\int_{S} k \nabla T \cdot \vec{n} \mathrm{~d} S-\int_{S} P \vec{V} \cdot \vec{n} \mathrm{~d} S+\int_{S} \tau \vec{u} \cdot \vec{n} \mathrm{~d} S
$$

a variável $k$ na integral do lado direito da equação é a condutividade térmica. Para um escoamento estacionário, sem forças de campo, sem troca de calor e invíscido, a equação de acima se reduz a:

$$
\int_{S}(E \vec{V} \cdot \vec{n}+P \vec{V} \cdot \vec{n}) \mathrm{d} S=0
$$

Pela definição de integral de superfície e pela Eq. (2.20), temos:

$$
\int_{A_{1}}\left(E_{1} \vec{V}_{1} \cdot \vec{n}_{1}+P_{1} \vec{V}_{1} \cdot \vec{n}_{1}\right) \mathrm{dA}+\int_{A_{2}}\left(E_{2} \vec{V}_{2} \cdot \vec{n}_{2}+P_{2} \vec{V}_{2} \cdot \vec{n}_{2}\right) \mathrm{dA}=0
$$

devido a que os integrandos são constantes e aplicando a Eq. (2.14), temos:

$$
\left[E_{1}\left(\vec{V}_{1} \cdot \vec{n}\right)+P_{1}\left(\vec{V}_{1} \cdot \vec{n}\right)\right] A_{1}=\left[E_{2}\left(\vec{V}_{2} \cdot \vec{n}\right)+P_{2}\left(\vec{V}_{2} \cdot \vec{n}\right)\right] A_{2}
$$

Aplicando Eqs. (2.26) e (2.16), temos:

$$
\left(E_{1}+P_{1}\right) W_{1}=\left(E_{2}+P_{2}\right) W_{2}
$$

Rearranjando o termo de densidade temos:

$$
\left(\frac{E_{1}}{\rho_{1}}+\frac{P_{1}}{\rho_{1}}\right) \rho_{1} W_{1}=\left(\frac{E_{2}}{\rho_{2}}+\frac{P_{2}}{\rho_{2}}\right) \rho_{2} W_{2}
$$

Pela conservação da massa em Eq. (2.31), tem-se:

$$
\frac{E_{1}}{\rho_{1}}+\frac{P_{1}}{\rho_{1}}=\frac{E_{2}}{\rho_{2}}+\frac{P_{2}}{\rho_{2}}
$$

Usando Eqs. (2.21) e (2.22) em Eq. (2.47), temos: 


$$
h_{1}+\frac{V_{1}^{2}}{2}=h_{2}+\frac{V_{2}^{2}}{2}
$$

Agora substituindo Eq. (2.19), temos:

$$
h_{1}+\frac{W_{1}^{2}+U_{1}^{2}}{2}=h_{2}+\frac{W_{2}^{2}+U_{2}^{2}}{2}
$$

Aplicando Eq. (2.18), temos:

$$
h_{1}+\frac{W_{1}^{2}}{2}=h_{2}+\frac{W_{2}^{2}}{2}
$$

Portanto temos a equação de conservação da energia para um volume de controle que contem uma descontinuidade.

- $\quad$ Equações de salto

As equações de salto são as formadas pelas equações de conservação e usam a notação compacta dada pela Eq. (2.12).

Equação de conservação da massa:

$$
[J]=0
$$

Equação de conservação da quantidade de movimento:

$$
[P+J W]=0
$$

Equação de conservação da energia:

$$
\left[h+\frac{W^{2}}{2}\right]=0
$$




\subsubsection{Equações de Rayleigh e Rankine-Hugoniot}

As equações de Rayleigh e as equações de Rankine-Hugoniot são obtidas basicamente rearranjando a equação de salto. Estas equações implicam unicamente em condições termodinâmicas, facilitando assim, uma interpretação analítica, geométrica e diferencial.

- Equação de Rayleigh

Substituindo a Eq. (2.51) na Eq. (2.52), e usando a notação de Eq. (2.13), temos:

$$
P_{1}-P_{2}=J\left(W_{2}-W_{1}\right)
$$

Isolando-se a velocidade normal em Eq. (2.24) para substituí-la na Eq. (2.54), logo pela Eq. (2.51) e notação de Eq. (2.13), temos a equação de Rayleigh, dado por:

$$
J^{2}=-\frac{[P]}{[v]}
$$

\section{- Equação de Rankine-Hugoniot (R-H)}

Isolando as velocidades normais da Eq. (2.24) para substituí-las na Eq. (2.53), e logo fazendo uso da Eq. (2.51) e da notação da Eq. (2.13), temos:

$$
h_{1}-h_{2}=\frac{\left(v_{2}-v_{1}\right)\left(v_{1}+v_{2}\right)}{2} J^{2}
$$

Agora substituindo Eq. (2.55) em Eq. (2.56) e fazendo uso da notação compacta, temos:

$$
[h]=[P] \frac{\left(v_{1}+v_{2}\right)}{2}
$$

\subsubsection{Solução de Chapman-Jouguet (C-J)}

Para encontrar a solução das equações de Rayleigh e de Rankine-Hugoniot, precisamos de mais uma equação, a saber, a equação de estado ou uma tabela termodinâmica. Como se pode perceber a equação de Rayleigh graficamente representa uma reta de inclinação negativa 
e a equação de R-H uma curva com concavidade para cima. A única forma de resolver Eqs. (2.55) e (2.57) é numericamente. De fato, as duas curvas se interceptam, inicialmente, em dois pontos, espera-se que exista uma interseção num único ponto, esse ponto será chamado de solução de Chapman-Jouguet, veja Fig. 2.4.

Buscamos o máximo da funcional $J^{2}$ (quadrado da vazão em massa por unidade de área). Matematicamente sabemos que uma condição necessária para que uma função alcance seus máximos locais ou mínimos locais é em pontos onde as derivadas parciais da função se anulam. Como $J^{2}$ é um funcional linear, nós usaremos o termo diferencial, ao invés das derivadas parciais. Então a diferencial de $J^{2}$ é dada por:

$$
d J^{2}=0
$$

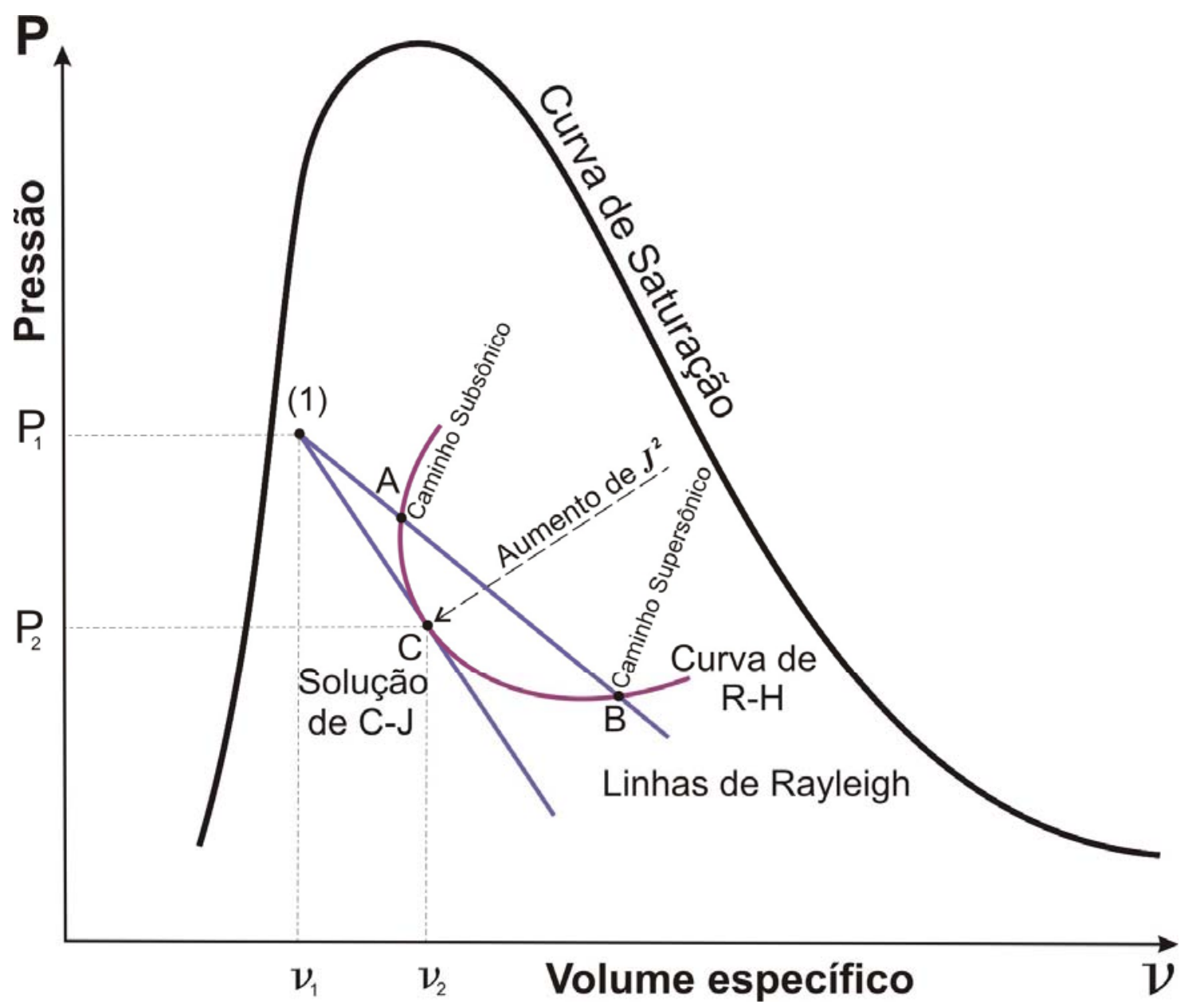

Figura 2.4 Comportamento esquemático do estado à montante de uma onda de evaporação (Simões-Moreira, 1994). 
Diferenciando temos:

$$
[P] d[v]-[v] d[P]=0
$$

como as propriedades do estado (1) são conhecidas e usando Eq. (2.55), temos:

$$
d v_{2}=-\frac{1}{J^{2}} d P_{2}
$$

$\mathrm{Ou}$

$$
\frac{d v_{2}}{d T_{2}}=-\frac{1}{J^{2}} \frac{d P_{2}}{d T_{2}}
$$

A Eq. (2.61) expressa nesta forma é valida, pois, a pressão e volume específico na fase líquida e vapor do estado bifásico são funções que dependem unicamente da temperatura, assim satisfazendo a hipótese do equilíbrio termodinâmico entre as fases.

Vamos, começar definindo o volume específico e a entalpia específica no estado (2), usando definição de Eq. (2.25):

$$
\begin{aligned}
& v_{2}=\left(1-x_{2}\right) v_{L 2}+x_{2} v_{V 2} \\
& h_{2}=\left(1-x_{2}\right) h_{L 2}+x_{2} h_{V 2}
\end{aligned}
$$

agora substituindo as Eqs. (2.62) e (2.63) na equação de R-H, dado pela Eq. (2.57), e isolando o título, temos:

$$
x_{2}=\frac{2\left(h_{1}-h_{L 2}\right)+[P]\left(v_{1}+v_{L 2}\right)}{2\left(h_{V 2}-h_{L_{2}}\right)-[P]\left(v_{V 2}+v_{L 2}\right)}
$$

Resolvendo-se numericamente as equações de Rayleigh e R-H, encontra-se a solução de C-J. Considera-se um valor inicial para a temperatura no estado (2), assim com aplicação da Tabela Termodinâmica conseguimos um valor para a pressão no mesmo estado, logo calculamos o título, segundo a Eq. (2.64). Com estas duas propriedades independentes $T_{2}, x_{2}$ e com a Eq. (2.61) conseguimos obter as solução de C-J e as outras propriedades termodinâmicas. O algoritmo de cálculo, em pseudocódigo, está apresentado na Fig. A.2 do Apêndice A. 


\section{4 Ângulos de Onda e de Giro}

Os ângulos de onda e de giro são importantes para o cálculo do vetor de velocidade no estado (2). Para a obtenção do ângulo de giro precisamos do valor do ângulo de onda e outras propriedades termodinâmicas do estado (2).

\subsection{1 Ângulo de onda}

Admitamos, como uma primeira aproximação, o formato do núcleo líquido de um cone com base na origem, como bem mostra a Fig. 2.5a, no sistema de coordenadas cartesianas $(y, x, z)$. Para um melhor estudo, consideremos este cone no sistema de coordenadas cilíndricas:

$$
\begin{aligned}
& y=r \cos (\vartheta) \\
& x=r \operatorname{sen}(\vartheta) \\
& z=z
\end{aligned}
$$

Onde $r$ é o raio da base do cone e $\vartheta$ o ângulo de rotação. A direção do escoamento que sai do bocal (base do cone) é paralela ao eixo "z" e "escapa" pela geratriz do cone, g. Cada seção longitudinal do cone tem o mesmo comportamento pelo qual o escoamento não depende da variação do ângulo, isto é: $\vartheta=0$. Ainda mais, cada secção longitudinal é simétrica com respeito ao eixo "z". Com estas simplificações a secção longitudinal de rotação do cone de nosso interesse, é um triângulo retângulo com base $L_{E}$ (comprimento da onda de evaporação), altura $R_{b}$ (raio da saída do bocal) e hipotenusa $g$, como se mostra na Fig. $2.5 b$. 


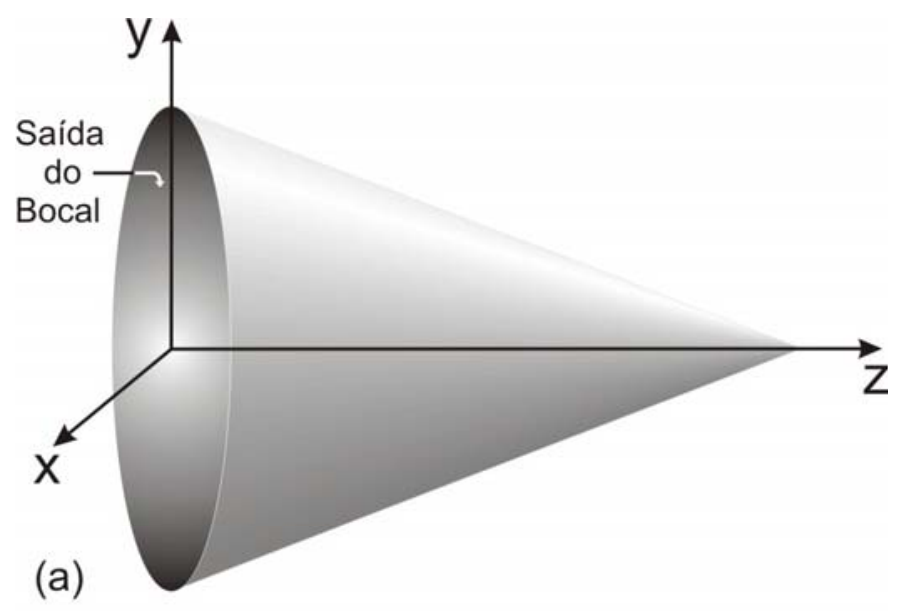

(b)

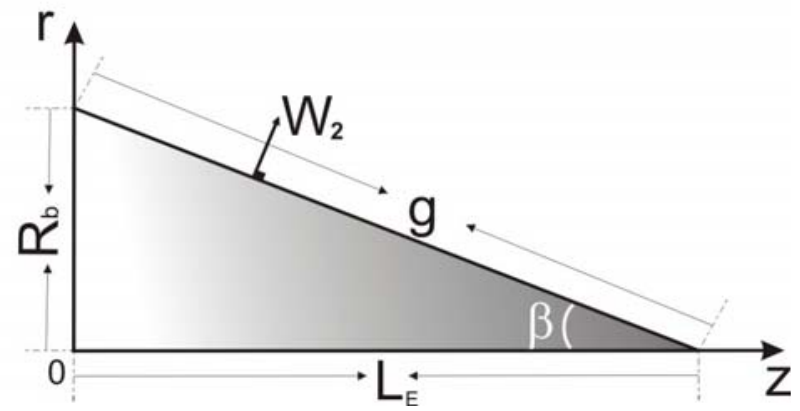

Figura 2.5 Formato do núcleo líquido cônico. (a) Superfície do núcleo líquido em 3D. (b) Secção longitudinal de rotação do cone ao redor do eixo " $z$ ".

O ângulo de onda denotado pela letra grega $\beta$, é a metade do vértice do cone. A seguir, explicaremos como se obtém uma fórmula para este ângulo de onda. Uma hipótese feita é a seguinte: "O fluxo de massa que passa pela saída do bocal é o mesmo que evapora na superfície do núcleo líquido”, isto é:

$$
\dot{m}_{e}=\dot{m}_{s}
$$

A vazão mássica que entra pela saída do bocal é igual ao valor fornecido por Vieira (2005).

$$
\dot{m}_{e}=\dot{m}
$$

A vazão em massa que deixa à superfície do núcleo líquido é dada por:

$$
\dot{m}_{s}=\frac{A l_{c} W_{2}}{v_{2}}
$$


onde a área lateral do cone é: $A l_{c}=\pi R_{b} \mathrm{~g} \operatorname{com} \mathrm{g}=\frac{R_{b}}{\operatorname{sen}(\beta)}$. Então substituindo Eqs. (2.67) e (2.68) em Eq. (2.66) obtemos uma fórmula para o ângulo de onda, dada por:

$$
\beta=\operatorname{arcsen}\left(\frac{\pi R_{b}^{2} W_{2}}{v_{2} \dot{m}}\right)
$$

\subsection{2 Ângulo de giro}

$\mathrm{O}$ ângulo de giro é o ângulo que forma o vetor de velocidade à montante e o vetor velocidade à jusante da descontinuidade. Neste estudo ocorre uma descontinuidade, que estará unicamente restrita à onda de evaporação (ver Fig. 2.6a) e à onda de choque (ver Fig. 2.6b), que são as que estão presentes na expansão de jatos altamente superaquecidos. O ângulo de giro será representado por a letra grega $\theta$ e é importante seu cálculo para as componentes do vetor de velocidade local. Continuando, deduziremos uma fórmula para o ângulo de giro, guiando-nos da Fig. 2.6.

De Eq. (2.18), temos:

$$
\begin{aligned}
\mathrm{U}_{1}=\mathrm{U}_{2} & \Leftrightarrow \\
\frac{W_{1}}{\operatorname{tg}(\beta)}=\frac{W_{2}}{\operatorname{tg}(\beta+\theta)} & \Leftrightarrow \\
\operatorname{tg}(\beta+\theta)=\frac{W_{2} \operatorname{tg}(\beta)}{W_{1}} & \Leftrightarrow \\
\frac{\operatorname{tg}(\beta)+\operatorname{tg}(\theta)}{1-\operatorname{tg}(\beta) \operatorname{tg}(\theta)}=\frac{W_{2} \operatorname{tg}(\beta)}{W_{1}} & \Leftrightarrow \\
\operatorname{tg}(\theta)=\frac{\operatorname{tg}(\beta)\left(\frac{W_{2}}{W_{1}}-1\right)}{1+\frac{W_{2}}{W_{1}} \operatorname{tg}^{2}(\beta)} &
\end{aligned}
$$




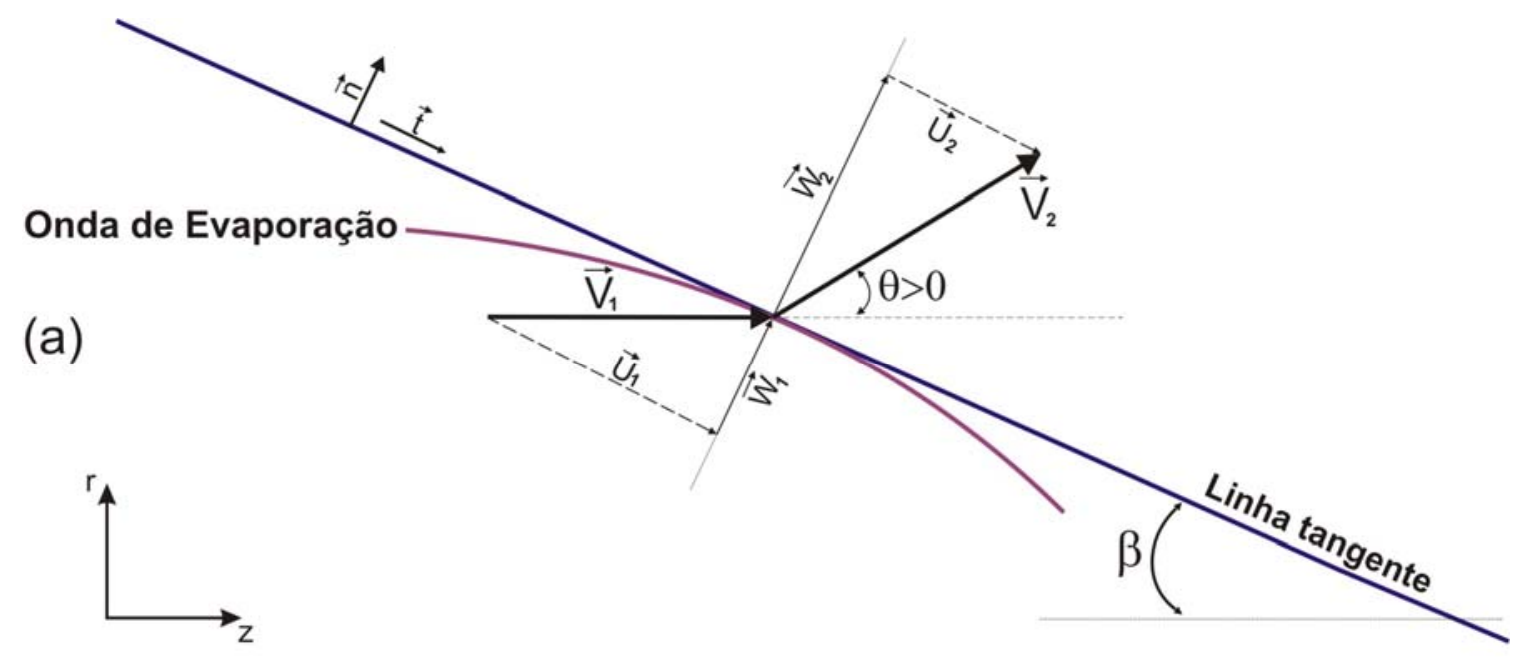

(b)

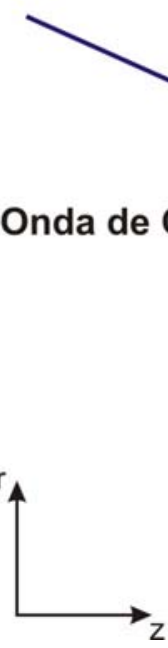

Figura 2.6 Ângulo de giro em uma descontinuidade. (a) Ângulo de giro para uma onda de evaporação. (b) Ângulo de giro para uma onda de choque.

Pela conservação da massa:

$$
\begin{array}{cc}
\frac{W_{2}}{W_{1}}=\frac{v_{2}}{v_{1}} & \Rightarrow \\
\operatorname{tg}(\theta)=\frac{\operatorname{tg}(\beta)\left(\frac{v_{2}}{v_{1}}-1\right)}{1+\frac{v_{2}}{v_{1}} \operatorname{tg}^{2}(\beta)} & \Leftrightarrow \\
\operatorname{tg}(\theta)=\frac{\operatorname{tg}(\beta)[v]}{v_{1}+[v] \operatorname{tg}^{2}(\beta)} & \Leftrightarrow
\end{array}
$$

Por tanto, o ângulo de giro é: 


$$
\theta=\operatorname{arctg}\left[\frac{\operatorname{tg}(\beta)[v]}{v_{1}+[v] \operatorname{tg}^{2}(\beta)}\right]
$$

\subsection{Formato do Núcleo Líquido}

Resultados experimentais de Vieira (1999, 2005), mostraram o formato do núcleo líquido cônico, veja Fig. 2.7. Do ponto de vista matemático, empregar um formato cônico para o núcleo introduz instabilidades numéricas, pois no vértice do cone nenhuma função é diferenciável. Consequentemente, isto se reflete no tratamento numérico. Por tal motivo, o formato do cone é suavizado no seu vértice, tomando agora um formato quase-paraboloidal, que é a junção de um tronco de cone com uma calota esférica, tratamento feito inicialmente por Angelo (2005), tal como se mostra a Fig. 2.8.

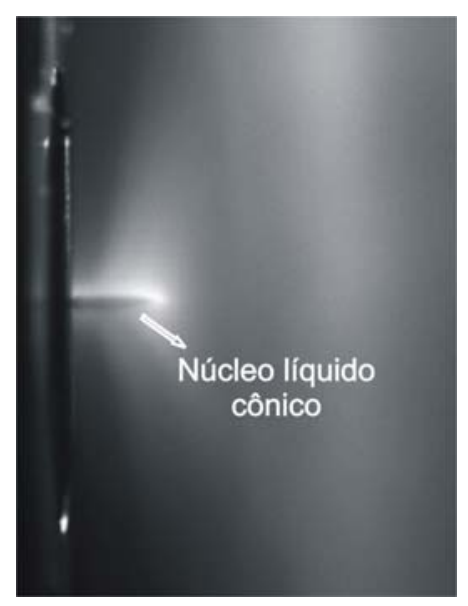

Figura 2.7 Foto do núcleo líquido cônico da Serie RUN3, ensaio 7404500, Vieira (2005).

\subsubsection{Novo raio do bocal}

Admitindo-se que o novo formato do cone líquido é quase-paraboloidal (um modelo esquemático é dado na Fig. 2.8a), consequentemente as variáveis geométricas variam, em particular, o raio do bocal. O raio do novo bocal será denotado por $R_{n b}$. Com ajuda da Fig. 2.8b explicaremos como obtê-lo. 
Defina-se $\varepsilon_{r}$ um valor pequeno da ordem (1.D-03 ou 1.D-04). O comprimento do "antigo" núcleo líquido (de formato cônico e fornecido experimentalmente) $L_{E}$, satisfaz:

$$
Z_{D}=L_{E}-d_{D C}-\varepsilon_{r}
$$

onde $z_{D}$ é comprimento do inicio da base do tronco piramidal até o centro da calota esférica. O raio da calota esférica, $d_{D C}$, é obtido trigonometricamente por:

$$
\operatorname{tg}(\beta)=\frac{R_{n b}-d_{D C} \cos (\beta)}{Z_{D}+d_{D C} \operatorname{sen}(\beta)}
$$

Substituindo Eq. (2.71) em Eq. (2.72) e isolando o termo $d_{D C}$, temos:

$$
d_{D C}=\frac{\operatorname{sen}(\beta)\left(\frac{R_{n b}}{\operatorname{tg}(\beta)}-\left(L_{E}-\varepsilon_{r}\right)\right)}{1-\operatorname{sen}(\beta)}
$$

O comprimento do núcleo líquido quase-paraboloidal, $L_{N}$ e é definido por:

$$
L_{N}=L_{E}+\varepsilon_{r}
$$

Voltando novamente à função $z_{D}$ na Eq. (2.71), vemos que é uma função que depende dos seguintes parâmetros: $L_{E}, R_{n b}, \beta$ e $\varepsilon_{r}$, isto é:

$$
z_{D}=z_{D}\left(\beta, L_{E}, R_{n b}, \varepsilon_{r}\right)
$$

Definem-se alguns comprimentos:

$$
\begin{aligned}
& L_{A E}=z_{D}+d_{D C} \operatorname{sen}(\beta) \\
& L_{B G}=R_{n b}-d_{D C} \cos (\beta) \\
& L_{B C}=\sqrt{L_{A E}^{2}+L_{B G}^{2}}
\end{aligned}
$$

A inclinação do tronco de cone é dada por:

$$
a_{m}=\frac{L_{B G}}{L_{A E}}
$$




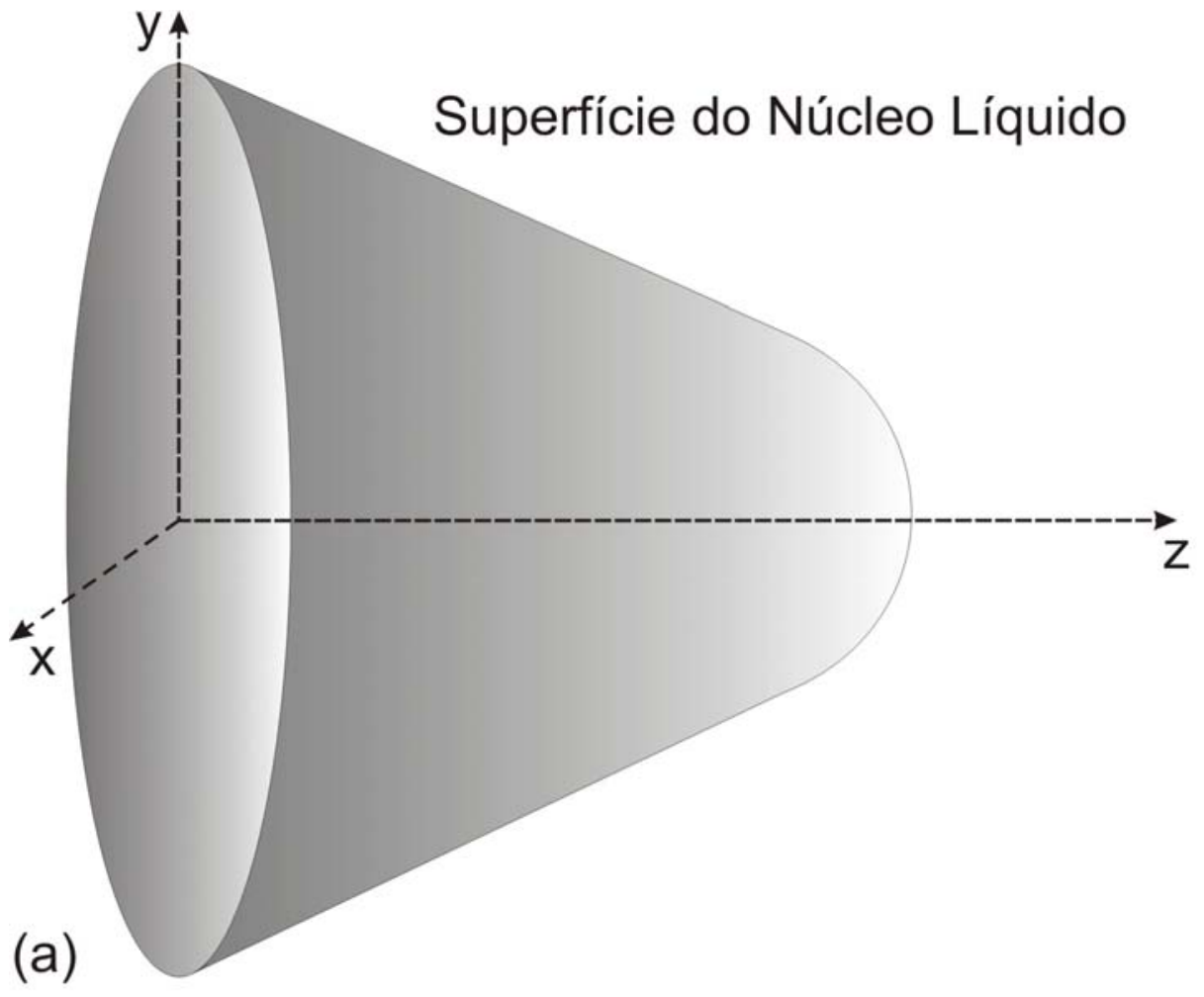

(b)

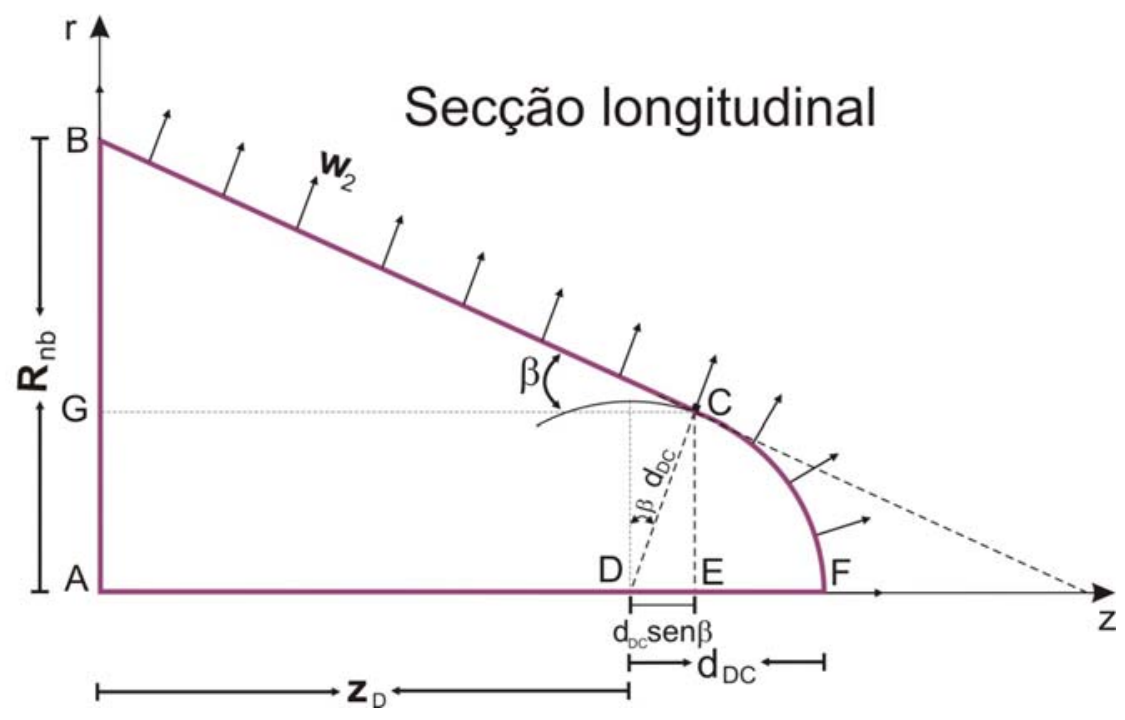

Figura 2.8 Formato esquemático do novo núcleo líquido quase-paraboloidal. (a) Superfície do núcleo líquido. (b) Secção longitudinal que gera o formato quase-paraboloidal do núcleo líquido.

- Área lateral do tronco de cone, $A_{\text {LTC }}$

Seja $f$ uma função definida no intervalo $[b, c]$, que descreve a curva da geratriz do tronco de cone:

$$
f(z)=a_{m} z+R_{n b} \Rightarrow f^{\prime}(z)=a_{m}
$$


Por definição, a área da superfície de revolução de uma curva ao redor do eixo “ $z$ ”, é:

$$
A_{L T C}=2 \pi \int_{b}^{c} f(z) \sqrt{1+\left[f^{\prime}(z)\right]^{2}} d z
$$

Portanto, a área lateral do tronco de cone em $\left[0, L_{A E}\right]$, é:

$$
A_{L T C}=\pi L_{B C}\left(2 R_{n b}-L_{B G}\right)
$$

- Área lateral da calota esférica, $A_{L C E}$

Seja $g$ uma função que descreve a curva da calota esférica:

$$
g(z)=\sqrt{d_{D C}^{2}-z^{2}} \Rightarrow g^{\prime}(z)=-\frac{z}{g(z)}
$$

Define-se o seguinte comprimento:

$$
L_{E F}=L_{N}-L_{A E}
$$

A área da superfície de revolução para esta calota esférica no intervalo $\left[0, L_{E F}\right]$, é:

$$
A_{L C E}=2 \pi d_{D C}^{2}[1-\operatorname{sen}(\beta)]
$$

- Área lateral do núcleo líquido quase-paraboloidal, $A_{L N N}$

A partir das Eqs. (2.80) e (2.83) obtemos a área lateral do núcleo líquido quaseparaboloidal:

$$
A_{L N N}=A_{L T C}+A_{L C E}
$$

- $\quad$ Novo valor para o raio do bocal, $\boldsymbol{R}_{n b}$

Pela conservação da massa, temos que a vazão em massa que entra pela saída do bocal é igual à vazão em massa que sai pela superfície do núcleo líquido, assim: 


$$
\dot{m}=\frac{A_{L N N} W_{2}}{v_{2}}
$$

onde $A_{L N N}$ é a área lateral do núcleo líquido quase-paraboloidal, cujo valor é dado na Eq. (2.84). Como se pode perceber esta última equação é uma função não linear de várias variáveis, isto é:

$$
F=F\left(R_{n b}, \beta, L_{E}, \dot{m}, v_{2}, W_{2}, \varepsilon_{r}\right)=\dot{m} v_{2}-A_{L N N} W_{2}=0
$$

A única variável que não é conhecida na Eq. (2.86) é o raio $R_{n b}$, então podemos dizer que temos uma função não linear que depende unicamente dessa variável. O raio $R_{n b}$ pode ser obtido numericamente, por exemplo, pelo método Newton Raphson. 


\section{EXPANSÃO BIFÁSICA}

Neste capítulo analisaremos a geometria da expansão bifásica no fenômeno de evaporação rápida de jatos de líquidos superaquecidos. Transformaremos o domínio físico em domínio computacional, fato importante quando se utiliza métodos numéricos de diferenças finitas. Finalmente abordaremos a formulação matemática das equações governantes em coordenadas cartesianas (ou cilíndricas) e coordenadas curvilíneas ortogonais.

\subsection{Domínio Físico}

A geometria da expansão bifásica de forma geral é uma região do espaço 3D de coordenadas cartesianas $(y, x, z)$. Devido ao sistema de coordenadas cilíndricas $(r, \vartheta, z)$ que se adotou para modelar esta geometria, veja Eq. (2.65), observamos que esta geometria é independente da variável angular $(\vartheta=0)$, sendo desta maneira considerada como uma região do plano 2D em coordenadas cilíndricas $(z, r)$. Além disso, é simétrica em relação ao eixo positivo “ $z$ ”. Esta geometria com estas simplificações é o que chamamos de domínio físico, veja Fig. 3.1a. Este domínio físico está limitado por linhas curvas, que chamaremos simplesmente de linhas:

Linha inicial: Segmento $\overline{A B}$. Para um melhor entendimento observe a linha $\overline{B F}$ na Fig. 2.8b.

$$
\begin{aligned}
\Gamma_{1} & =\Gamma_{1 A} \cup \Gamma_{1 B} \\
\Gamma_{1 A} & =\left\{(z, r): 0 \leq z \leq L_{A E}, \quad r=a_{m} z+R_{n b}\right\} \\
\Gamma_{1 B} & =\left\{(z, r): L_{A E} \leq z \leq R_{n b}, r=\sqrt{d_{D C}^{2}-z^{2}}\right\}
\end{aligned}
$$

Linha de simetria: Segmento $\overline{B C}$.

$$
\Gamma_{2}=\left\{(z, r): L_{N} \leq z \leq R_{f}, r=0\right\}
$$

onde $R_{f}$ é o raio de uma circunferência. 
Linha ao longe: Curva $\overline{C D}$. Em 2D é a quarta parte de uma circunferência de raio $R_{f}$.

$$
\Gamma_{3}=\left\{(z, r): 0 \leq z \leq R_{f}, r=\sqrt{R_{f}^{2}-z^{2}}\right\}
$$

Linha da parede: Segmento $\overline{A D}$.

$$
\Gamma_{4}=\left\{(z, r): z=0, \quad 0 \leq r \leq R_{f}\right\}
$$

Com isto o domínio físico está limitado pelo seguinte contorno:

$$
\Gamma=\bigcup_{i=1}^{4} \Gamma_{i}
$$
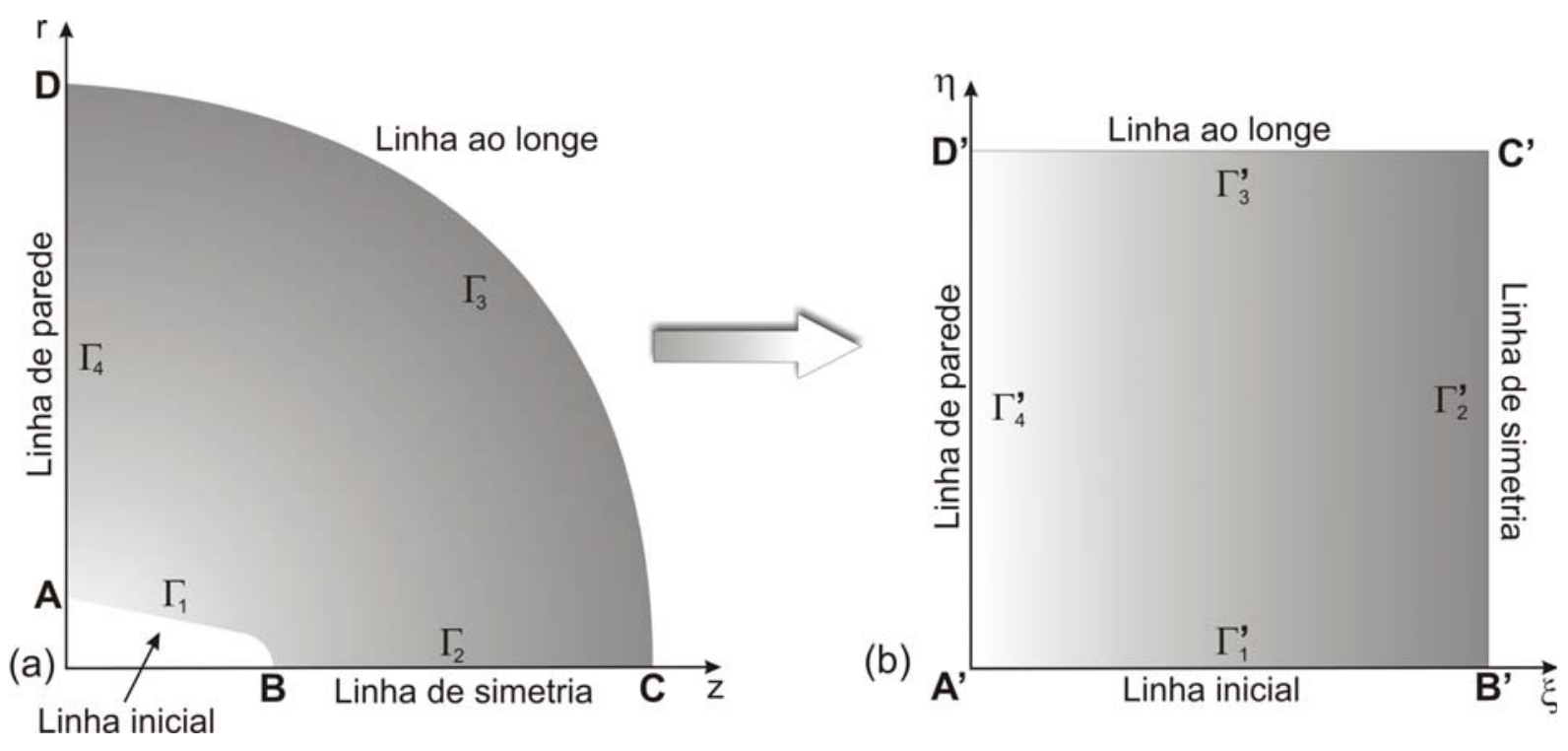

Figura 3.1 Domínio da expansão bifásica. a) Domínio físico $\Gamma$ de raio $R_{f}$. b) Domínio computacional $\Gamma^{\prime}$.

\subsection{Domínio Computacional}

O domínio computacional é o quadrado unitário 2D nas coordenadas curvilíneas ortogonais $(\xi, \eta)$. Na Fig. 3.1b podemos ver que o domínio computacional é limitado pelas seguintes linhas curvas, que chamaremos simplesmente de linhas:

Linha inicial: Segmento $\overline{A^{\prime} B^{\prime}}$. 


$$
\Gamma_{1}^{\prime}=\{(\xi, \eta): \xi=0,0 \leq \eta \leq 1\}
$$

Linha de simetria: Segmento $\overline{B^{\prime} C^{\prime}}$.

$$
\Gamma_{2}^{\prime}=\{(\xi, \eta): 0 \leq \xi \leq 1, \eta=0\}
$$

Linha ao longe: Segmento $\overline{D^{\prime} C^{\prime}}$.

$$
\Gamma_{3}^{\prime}=\{(\xi, \eta): \xi=1,0 \leq \eta \leq 1\}
$$

Linha de parede: Segmento $\overline{A^{\prime} D^{\prime}}$.

$$
\Gamma_{4}^{\prime}=\{(\xi, \eta): 0 \leq \xi \leq 1, \eta=1\}
$$

Por tanto, o domínio computacional está limitado pelo seguinte contorno:

$$
\Gamma^{\prime}=\bigcup_{i=1}^{4} \Gamma_{i}^{\prime}
$$

\subsection{Condições de Contorno}

No domínio físico as condições de contorno são conhecidas basicamente na Linha inicial (todas as propriedades termodinâmicas) e na Linha ao longe (unicamente a pressão ao longe, “far field” na literatura inglesa). O fato de que o escoamento é bifásico neste domínio, implica que a pressão e a temperatura são dependentes. Então as duas propriedades termodinâmicas independentes podem ser $\{p, x\}$ ou $\{T, x\}$.

$\operatorname{Em} \Gamma_{1}:$

$$
\begin{array}{ll}
V_{z} \neq 0 & p=p_{2} \\
V_{r} \neq 0 & x=x_{2} \\
\text { Mach }=1 &
\end{array}
$$


$\operatorname{Em} \Gamma_{2}:$

$$
\begin{aligned}
& V_{z} \neq 0 \\
& V_{r}=0
\end{aligned}
$$

$\operatorname{Em} \Gamma_{3}:$

$$
p=p_{\infty}
$$

$\operatorname{Em~} \Gamma_{4}$ :

$$
\begin{aligned}
& V_{z}=0 \\
& V_{r} \neq 0
\end{aligned}
$$

\subsection{Geração de Malha Estruturada}

\subsubsection{Transformação de coordenadas}

Um domínio computacional é aquele que resulta do domínio físico via uma transformação de coordenadas biunívoca. Considere $f$ uma função de variável complexa que transforma o plano físico no plano computacional:

$$
f=\xi+i \eta
$$

Onde $\xi$ e $\eta$ são, respectivamente a parte real e a parte imaginária, dadas por:

$$
\begin{aligned}
& \xi=\xi(z, r) \\
& \eta=\eta(z, r)
\end{aligned}
$$

Da Eq. (3.16) podemos dizer que $(z, r)$ são as variáveis independentes e $(\xi, \eta)$ as variáveis dependentes. As variáveis $(\xi, \eta)$ são as coordenadas ortogonais conhecidas num quadrado unitário. 
Se $f$ é uma Transformação Conforme, pela teoria de análise complexa (veja Lins (1996)) esta transformação (parte real e parte imaginária) preserva a equação de Laplace, isto é:

$$
\left\{\begin{aligned}
\Delta \xi & =0 \\
\Delta \eta & =0
\end{aligned}\right.
$$

Defina-se a condição de contorno de Dirichlet, como:

$$
\left.\xi\right|_{\Gamma}=\xi_{0},\left.\quad \eta\right|_{\Gamma}=\eta_{0}
$$

onde $\xi_{0}$ e $\eta_{0}$ são valores conhecidos no contorno do quadrado unitário. Como nosso objetivo é conhecer as coordenadas $(z, r)$ do domínio físico, então precisamos levar o problema de contorno, formulados pela Eqs. (3.17) e (3.18), a um sistema onde as variáveis dependentes sejam $(z, r)$. Depois de alguns cálculos diferenciais, temos o problema equivalente:

$$
\left\{\begin{array}{l}
g_{22} \frac{\partial^{2} z}{\partial \xi^{2}}-2 g_{12} \frac{\partial^{2} z}{\partial \xi \partial \eta}+g_{11} \frac{\partial^{2} z}{\partial \eta^{2}}=0 \\
g_{22} \frac{\partial^{2} r}{\partial \xi^{2}}-2 g_{12} \frac{\partial^{2} r}{\partial \xi \partial \eta}+g_{11} \frac{\partial^{2} r}{\partial \eta^{2}}=0
\end{array}\right.
$$

Onde

$$
g_{12}=\frac{\partial z}{\partial \xi} \frac{\partial z}{\partial \eta}+\frac{\partial r}{\partial \xi} \frac{\partial r}{\partial \eta}, \quad g_{11}=\left(\frac{\partial z}{\partial \xi}\right)^{2}+\left(\frac{\partial r}{\partial \xi}\right)^{2}, \quad g_{22}=\left(\frac{\partial z}{\partial \eta}\right)^{2}+\left(\frac{\partial r}{\partial \eta}\right)^{2}
$$

e condições de contorno de Dirichlet:

$$
\left.z\right|_{\Upsilon}=z_{0},\left.\quad r\right|_{\Upsilon}=r_{0}
$$

onde $z_{0}$ e $r_{0}$ são valores conhecidos no contorno do domínio físico.

Para resolver o problema de contorno elíptico, formulados pelas Eqs. (3.19), (3.20) e (3.21), usaremos métodos numéricos de diferenças finitas, e métodos de sub-relaxação para o sistema algébrico não linear. 


\subsubsection{Malha física e computacional}

A malha física na Fig. 3.2a, é do tipo estruturada, e seus nós são obtidos pela solução do problema de valor de contorno de Dirichlet, dada pelas Eqs. (3.19), (3.20) e (3.21). A malha computacional na Fig. 3.2b, é do tipo retangular e estão no plano $(\xi, \eta)$, onde todos seus nós são conhecidos e é nesta malha que o método de diferenças finitas será aplicado.
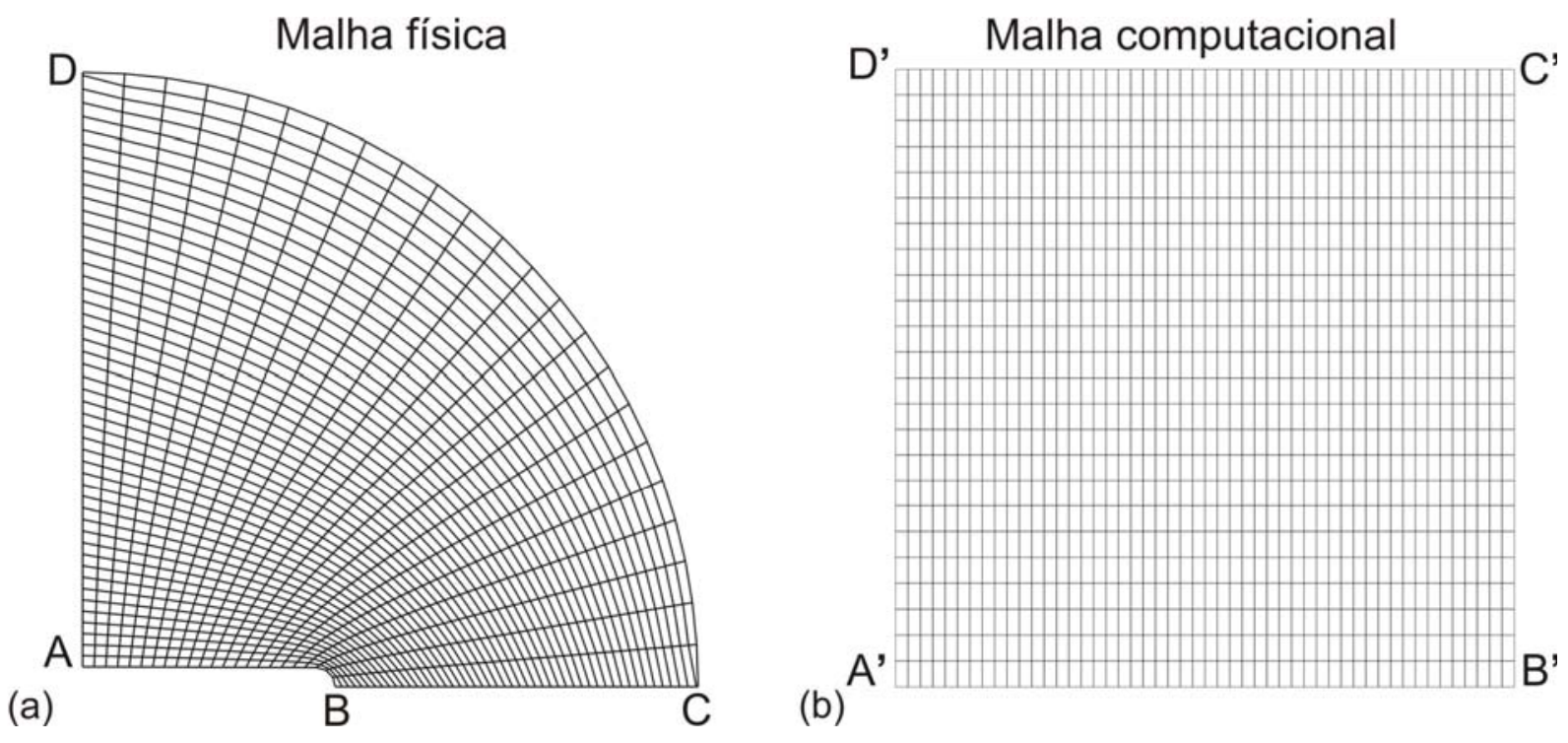

Figura 3.2 (a) Malha Física de raio $R_{f}$. (b) Malha Computacional.

\subsection{Formulação Matemática das Equações Governantes}

Existem dois tipos de formulações para um sistema de equações em derivadas parciais, a formulação conservativa e a formulação não conservativa. Segundo Anderson (1995), a formulação conservativa é mais eficiente se comparada à formulação não conservativa do ponto de vista computacional. Nesta seção estudaremos a formulação conservativa em coordenadas cartesianas e em coordenadas curvilíneas ortogonais.

\subsubsection{Em coordenadas cartesianas}

A formulação conservativa, conhecida em outras literaturas como Leis de conservação, é definida como um sistema de equações em derivadas parciais quase-lineares sob a forma particular de divergência. Nesta formulação são envolvidas variáveis de estado (variáveis de fluxo) e funções fluxos. 
As equações que governam o comportamento dos fluidos não estacionários e invíscidos numa região axissimétrica 2D são:

Conservação da massa:

$$
\frac{\partial}{\partial t} \rho+\frac{\partial}{\partial x}(\rho \mathrm{u})+\frac{\partial}{\partial y}(\rho \mathrm{v})=-\frac{1}{y} \rho \mathrm{v}
$$

Conservação da quantidade de movimento, na direção $x$ :

$$
\frac{\partial}{\partial t}(\rho \mathrm{u})+\frac{\partial}{\partial x}\left(\rho \mathrm{u}^{2}+p\right)+\frac{\partial}{\partial y}(\rho \mathrm{uv})=-\frac{1}{y} \rho \mathrm{uv}
$$

Conservação da quantidade de movimento, na direção $y$ :

$$
\frac{\partial}{\partial t}(\rho \mathrm{v})+\frac{\partial}{\partial x}(\rho \mathrm{uv})+\frac{\partial}{\partial y}\left(\rho \mathrm{v}^{2}+p\right)=-\frac{1}{y} \rho \mathrm{v}^{2}
$$

Conservação da energia:

$$
\frac{\partial}{\partial t} E+\frac{\partial}{\partial x}[(E+p) \mathrm{u}]+\frac{\partial}{\partial y}[(E+p) \mathrm{v}]=-\frac{1}{y}(E+p) \mathrm{v}
$$

Este sistema formado por quatro equações apresenta, do ponto de vista não conservativo, cinco incógnitas ou variáveis primitivas, a saber: $\{\rho, \mathrm{u}, \mathrm{v}, p, e\}$ densidade, vetor velocidade na direção $x$, vetor velocidade na direção $y$, pressão e energia interna específica, respectivamente. Esta energia interna específica satisfaz:

$$
E=\rho e+\frac{1}{2} \rho V^{2}
$$

onde $E$ é a energia total.

Fica claro que está faltando mais uma equação para que o sistema seja completo, esta equação é uma equação de estado, ou seja, uma equação que relacione a pressão, a temperatura e ou volume específico:

$$
F(p, T, v)=0
$$

Para o caso de gases ideais temos:

$$
p v=R T, \quad R=\frac{\bar{R}}{M}
$$

Onde $\bar{R}$ é a constante universal dos gases perfeitos e $M$ massa molecular. 
Em nosso estudo usaremos a equação de estado de Lee-Kesler (mais claramente a Tabela das propriedades termodinâmicas na saturação do iso-octano). Esta equação de estado é não linear e, portanto mais complexa, pois, é dada para fluidos “reais” o qual dificulta a formulação matemática do fenômeno, para mais detalhes veja Apêndice D.

Rearranjando termos em Eqs. (3.22)-(3.25) e fazendo uso de notação vetorial obtemos a seguinte Lei de conservação:

$$
\frac{\partial U}{\partial t}+\frac{\partial F}{\partial x}+\frac{\partial G}{\partial y}+\frac{S}{y}=0
$$

Onde:

$$
U=\left[\begin{array}{c}
\rho \\
\rho \mathrm{u} \\
\rho \mathrm{v} \\
E
\end{array}\right] \quad F=\left[\begin{array}{c}
\rho \mathrm{u} \\
\rho \mathrm{u}^{2}+p \\
\rho \mathrm{uv} \\
(E+p) \mathrm{u}
\end{array}\right] \quad G=\left[\begin{array}{c}
\rho \mathrm{v} \\
\rho \mathrm{uv} \\
\rho \mathrm{v}^{2}+p \\
(E+p) \mathrm{v}
\end{array}\right] \quad S=\left[\begin{array}{c}
\rho \mathrm{v} \\
\rho \mathrm{uv} \\
\rho \mathrm{v}^{2} \\
(E+p) \mathrm{v}
\end{array}\right]
$$

Chamamos a: $U$ vetor incógnita das variáveis de estado (variáveis de fluxo), $F$ e $G$ funções vetoriais de fluxo e $S$ termo fonte.

\subsubsection{Em coordenadas curvilíneas ortogonais}

As equações básicas em dinâmica dos fluidos são válidas para qualquer sistema de coordenadas. As coordenadas curvilíneas ortogonais são denotadas em espaço 2D por $(\xi, \eta)$. Considere a seguinte transformação de coordenadas:

$$
T:\left\{\begin{array}{l}
\xi=\xi(x, y, t) \\
\eta=\eta(x, y, t) \\
\tau=t
\end{array}\right.
$$

Onde $T$ é a transformação das coordenadas $(x, y, z)$ em $(\xi, \eta, \tau)$, e admita-se que sua inversa exista e denotada por $T^{-1}$. Segundo o gráfico na Fig. $3.3 f$, uma função qualquer, está definida no plano $(x, y)$ e $\bar{f}$ no plano $(\xi, \eta) \cdot R^{n}$ indica a imagem de $f$ que pode ser um escalar, campo ou tensor. Então temos: 


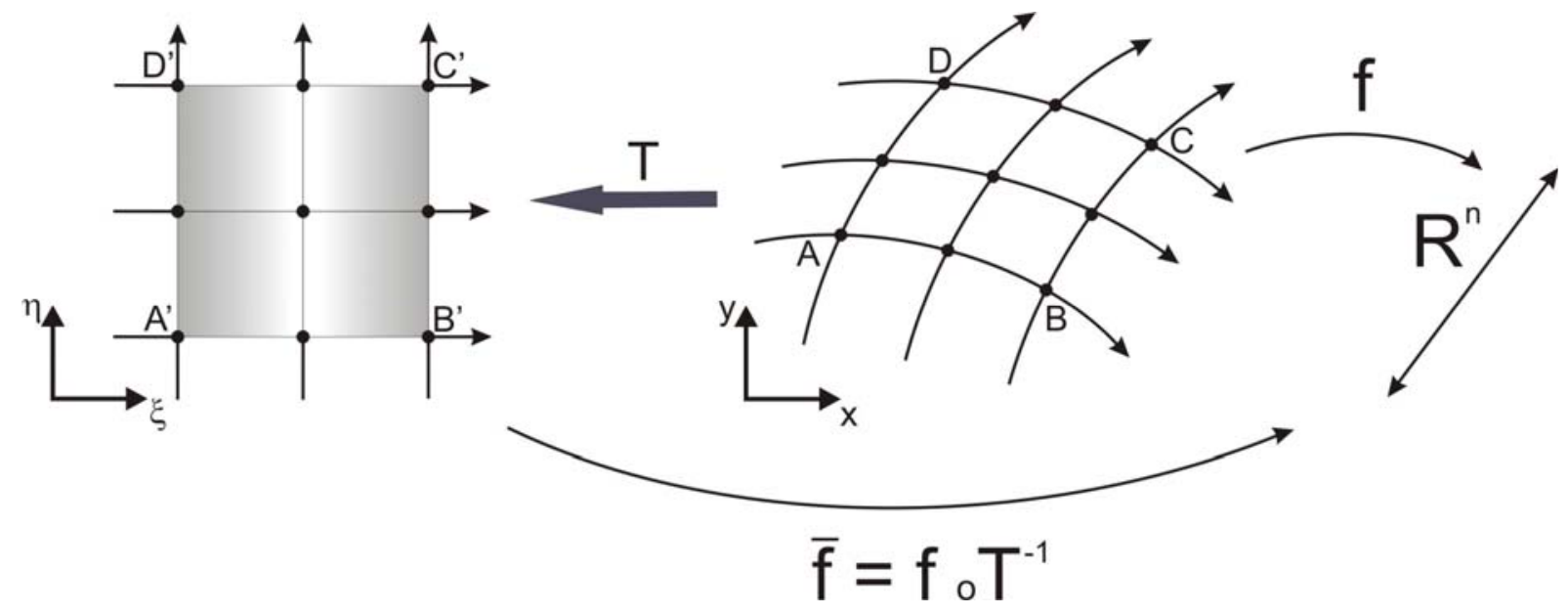

Figura 3.3 Transformação de coordenadas.

$$
\begin{aligned}
\bar{f}(\xi, \eta, \tau) & =\left[f \circ T^{-1}\right](x, y, t) \\
& =f\left[T^{-1}(x, y, t)\right] \\
& =f(x, y, t)
\end{aligned}
$$

Defina-se a matriz Jacobiana da transformação $T$ :

$$
J_{a}=\frac{\partial(\xi, \eta, \tau)}{\partial(x, y, t)}=\left[\begin{array}{ccc}
\xi_{x} & \xi_{y} & \xi_{t} \\
\eta_{x} & \eta_{y} & \eta_{t} \\
\tau_{x} & \tau_{y} & \tau_{t}
\end{array}\right]=\left[\begin{array}{ccc}
\xi_{x} & \xi_{y} & \xi_{t} \\
\eta_{x} & \eta_{y} & \eta_{t} \\
0 & 0 & 1
\end{array}\right]
$$

Para um domínio fixo ou sem movimento, temos que:

$$
\xi_{t}=\eta_{t}=0
$$

Então

$$
J_{a}=\left[\begin{array}{ccc}
\xi_{x} & \xi_{y} & 0 \\
\eta_{x} & \eta_{y} & 0 \\
0 & 0 & 1
\end{array}\right]
$$

Defina-se o Jacobiano:

$$
J=\operatorname{det}\left(J_{a}\right)=\xi_{x} \eta_{y}-\xi_{y} \eta_{x}
$$

Por outro lado diferenciando a transformação dada em Eq. (3.31), temos: 


$$
\begin{aligned}
& d \xi=\xi_{x} d x+\xi_{y} d y \\
& d \eta=\eta_{x} d x+\eta_{y} d y \\
& d \tau=d t
\end{aligned} \quad \Leftrightarrow\left[\begin{array}{l}
d \xi \\
d \eta \\
d \tau
\end{array}\right]=J_{a}\left[\begin{array}{l}
d x \\
d y \\
d t
\end{array}\right]
$$

Calculando a inversa da matriz Jacobiana:

$$
J_{a}^{-1}=\frac{1}{J}[\operatorname{Adj}(A)]^{t}=\frac{1}{J}\left[\begin{array}{ccc}
A_{11} & A_{21} & A_{31} \\
A_{12} & A_{22} & A_{32} \\
A_{13} & A_{23} & A_{33}
\end{array}\right]
$$

Fazendo os cálculos dos elementos da matriz adjunta de $A$, temos:

$$
J_{a}^{-1}=\frac{1}{J}\left[\begin{array}{ccc}
\eta_{y} & -\xi_{y} & 0 \\
-\eta_{x} & \xi_{x} & 0 \\
0 & 0 & J
\end{array}\right]
$$

De Eq.(3.37), temos:

Por Eq. (3.39):

$$
\left[\begin{array}{l}
d x \\
d y \\
d t
\end{array}\right]=J_{a}^{-1}\left[\begin{array}{l}
d \xi \\
d \eta \\
d \tau
\end{array}\right]
$$

$$
\begin{aligned}
d x & =\frac{1}{J}\left[\eta_{y} d \xi-\xi_{y} d \eta\right] \\
d y & =\frac{1}{J}\left[-\eta_{x} d \xi+\xi_{x} d \eta\right] \\
d t & =\frac{1}{J}[J d \tau]=d \tau
\end{aligned}
$$

Devido à existência da inversa da transformação temos:

$$
\begin{aligned}
x & =x(\xi, \eta, \tau) \\
y & =y(\xi, \eta, \tau) \\
t & =\tau
\end{aligned}
$$

Aplicando a definição de uma diferencial na Eq. (3.42):

$$
\begin{aligned}
& d x=x_{\xi} d \xi+x_{\eta} d \eta \\
& d y=y_{\xi} d \xi+y_{\eta} d \eta \\
& d t=d \tau
\end{aligned}
$$

Igualando Eqs. (3.41) e (3.43), temos as chamadas métricas: 


$$
\begin{array}{|ll}
\xi_{x}=J y_{\eta} & \xi_{y}=-J x_{\eta} \\
\eta_{x}=-J y_{\xi} & \eta_{y}=J x_{\xi}
\end{array}
$$

Substituindo na definição do Jacobiano dada pela Eq. (3.36), temos:

$$
\begin{gathered}
J=\xi_{x} \eta_{y}-\xi_{y} \eta_{x}=J y_{\eta} J x_{\xi}-\left(-J x_{\eta}\right)\left(-J y_{\xi}\right) \Leftrightarrow \\
J=J^{2}\left(y_{\eta} x_{\xi}-x_{\eta} y_{\xi}\right) \Leftrightarrow \\
J=\frac{1}{x_{\xi} y_{\eta}-x_{\eta} y_{\xi}}
\end{gathered}
$$

Onde cada uma das componentes do jacobiano é aproximada por diferenças finitas, neste caso por diferenças finitas centrais, assim:

$$
\begin{array}{ll}
x_{\xi}=\frac{x_{i+1}-x_{i-1}}{2 \Delta \xi} & x_{\eta}=\frac{x_{j+1}-x_{j-1}}{2 \Delta \eta} \\
y_{\xi}=\frac{y_{i+1}-y_{i-1}}{2 \Delta \xi} & y_{\eta}=\frac{y_{j+1}-y_{j-1}}{2 \Delta \eta}
\end{array}
$$

Onde $\Delta \xi$ é o comprimento na direção $\xi$ e $\Delta \eta$ o comprimento na direção $\eta$ de um elemento do domínio computacional.

Uma vez estabelecidas as fórmulas das métricas e do jacobiano, procedemos à transformação do sistema de equações:

$$
\frac{\partial U}{\partial t}+\frac{\partial F}{\partial x}+\frac{\partial G}{\partial y}+\frac{S}{y}=0
$$

Calculando cada termo:

$$
\begin{aligned}
\frac{\partial U}{\partial t} & =\frac{\partial \bar{U}}{\partial \xi} \xi_{t}+\frac{\partial \bar{U}}{\partial \eta} \not \eta_{t}+\frac{\partial \bar{U}}{\partial \tau} \tau_{t}=\frac{\partial \bar{U}}{\partial \tau} \\
\frac{\partial F}{\partial x} & =\frac{\partial \bar{F}}{\partial \xi} \xi_{x}+\frac{\partial \bar{F}}{\partial \eta} \eta_{x}+\frac{\partial \bar{F}}{\partial \tau} \tau / x=\frac{\partial \bar{F}}{\partial \xi} \xi_{x}+\frac{\partial \bar{F}}{\partial \eta} \eta_{x} \\
\frac{\partial G}{\partial y} & =\frac{\partial \bar{G}}{\partial \xi} \xi_{y}+\frac{\partial \bar{G}}{\partial \eta} \eta_{y}+\frac{\partial \bar{G}}{\partial \tau} \tau / y=\frac{\partial \bar{G}}{\partial \xi} \xi_{y}+\frac{\partial \bar{G}}{\partial \eta} \eta_{y} \\
\frac{S}{y} & =\frac{\bar{S}}{y}
\end{aligned}
$$


Substituindo, temos:

$$
\frac{\partial U}{\partial t}+\frac{\partial F}{\partial x}+\frac{\partial G}{\partial y}+\frac{S}{y}=\frac{\partial \bar{U}}{\partial \tau}+\frac{\partial \bar{F}}{\partial \xi} \xi_{x}+\frac{\partial \bar{F}}{\partial \eta} \eta_{x}+\frac{\partial \bar{G}}{\partial \xi} \xi_{y}+\frac{\partial \bar{G}}{\partial \eta} \eta_{y}+\frac{\bar{S}}{y}=0
$$

Para facilitar cálculos consideremos as seguintes notações:

$$
U=\bar{U}, \quad F=\bar{F}, \ldots
$$

Além disso, sabemos que:

$$
\tau=t
$$

Logo temos:

$$
\frac{\partial U}{\partial t}+\frac{\partial F}{\partial \xi} \xi_{x}+\frac{\partial F}{\partial \eta} \eta_{x}+\frac{\partial G}{\partial \xi} \xi_{y}+\frac{\partial G}{\partial \eta} \eta_{y}+\frac{S}{y}=0
$$

Dividendo entre o Jacobiano tem-se:

$$
\frac{1}{J} \frac{\partial U}{\partial t}+\xi_{x} \frac{1}{J} \frac{\partial F}{\partial \xi}+\eta_{x} \frac{1}{J} \frac{\partial F}{\partial \eta}+\xi_{y} \frac{1}{J} \frac{\partial G}{\partial \xi}+\eta_{y} \frac{1}{J} \frac{\partial G}{\partial \eta}+\frac{1}{J} \frac{S}{y}=0
$$

Equivalentemente:

$$
\frac{\partial}{\partial t}\left(\frac{U}{J}\right)+\frac{\xi_{x}}{J} \frac{\partial F}{\partial \xi}+\frac{\eta_{x}}{J} \frac{\partial F}{\partial \eta}+\frac{\xi_{y}}{J} \frac{\partial G}{\partial \xi}+\frac{\eta_{y}}{J} \frac{\partial G}{\partial \eta}+\frac{S}{J y}=0
$$

Sabemos que:

$$
\begin{aligned}
& \frac{\xi_{x}}{J} \frac{\partial F}{\partial \xi}=\frac{\partial}{\partial \xi}\left(\frac{\xi_{x}}{J} F\right)-F \frac{\partial}{\partial \xi}\left(\frac{\xi_{x}}{J}\right) \\
& \frac{\eta_{x}}{J} \frac{\partial F}{\partial \eta}=\frac{\partial}{\partial \eta}\left(\frac{\eta_{x}}{J} F\right)-F \frac{\partial}{\partial \eta}\left(\frac{\eta_{x}}{J}\right) \\
& \frac{\xi_{y}}{J} \frac{\partial G}{\partial \xi}=\frac{\partial}{\partial \xi}\left(\frac{\xi_{y}}{J} G\right)-G \frac{\partial}{\partial \xi}\left(\frac{\xi_{y}}{J}\right) \\
& \frac{\eta_{y}}{J} \frac{\partial G}{\partial \eta}=\frac{\partial}{\partial \eta}\left(\frac{\eta_{y}}{J} G\right)-G \frac{\partial}{\partial \eta}\left(\frac{\eta_{y}}{J}\right)
\end{aligned}
$$

Substituindo em Eq. (3.54): 


$$
\begin{aligned}
& \frac{\partial}{\partial t}\left(\frac{U}{J}\right)+\frac{\partial}{\partial \xi}\left(\frac{\xi_{x}}{J} F\right)-F \frac{\partial}{\partial \xi}\left(\frac{\xi_{x}}{J}\right)+\frac{\partial}{\partial \eta}\left(\frac{\eta_{x}}{J} F\right)-F \frac{\partial}{\partial \eta}\left(\frac{\eta_{x}}{J}\right)+ \\
& \frac{\partial}{\partial \xi}\left(\frac{\xi_{y}}{J} G\right)-G \frac{\partial}{\partial \xi}\left(\frac{\xi_{y}}{J}\right)+\frac{\partial}{\partial \eta}\left(\frac{\eta_{y}}{J} G\right)-G \frac{\partial}{\partial \eta}\left(\frac{\eta_{y}}{J}\right)+\frac{S}{J y}=0
\end{aligned}
$$

Rearranjando a equação obtemos:

$$
\begin{aligned}
& \frac{\partial}{\partial t}\left(\frac{U}{J}\right)+\frac{\partial}{\partial \xi}\left(\frac{\xi_{x}}{J} F+\frac{\xi_{y}}{J} G\right)+\frac{\partial}{\partial \eta}\left(\frac{\eta_{x}}{J} F+\frac{\eta_{y}}{J} G\right)+\frac{S}{J y} \\
& -F\left[\frac{\partial}{\partial \xi}\left(\frac{\xi_{x}}{J}\right)+\frac{\partial}{\partial \eta}\left(\frac{\eta_{x}}{J}\right)\right]-G\left[\frac{\partial}{\partial \xi}\left(\frac{\xi_{y}}{J}\right)+\frac{\partial}{\partial \eta}\left(\frac{\eta_{y}}{J}\right)\right]=0
\end{aligned}
$$

Veja que cada termo entre colchete se anula:

$$
\begin{aligned}
& {\left[\frac{\partial}{\partial \xi}\left(\frac{\xi_{x}}{J}\right)+\frac{\partial}{\partial \eta}\left(\frac{\eta_{x}}{J}\right)\right]=\frac{\partial}{\partial \xi}\left(y_{\eta}\right)-\frac{\partial}{\partial \eta}\left(y_{\xi}\right)=\frac{\partial y_{\eta}}{\partial \xi}-\frac{\partial y_{\eta}}{\partial \xi}=0} \\
& {\left[\frac{\partial}{\partial \xi}\left(\frac{\xi_{y}}{J}\right)+\frac{\partial}{\partial \eta}\left(\frac{\eta_{y}}{J}\right)\right]=-\frac{\partial}{\partial \xi}\left(x_{\eta}\right)+\frac{\partial}{\partial \eta}\left(x_{\xi}\right)=-\frac{\partial x_{\eta}}{\partial \xi}+\frac{\partial x_{\eta}}{\partial \xi}=0}
\end{aligned}
$$

Então temos:

$$
\frac{\partial}{\partial t}\left(\frac{U}{J}\right)+\frac{\partial}{\partial \xi}\left(\frac{\xi_{x}}{J} F+\frac{\xi_{y}}{J} G\right)+\frac{\partial}{\partial \eta}\left(\frac{\eta_{x}}{J} F+\frac{\eta_{y}}{J} G\right)+\frac{S}{J y}=0
$$

Equivalentemente:

$$
\frac{\partial}{\partial t}\left(\frac{U}{J}\right)+\frac{\partial}{\partial \xi}\left[\frac{1}{J}\left(\xi_{x} F+\xi_{y} G\right)\right]+\frac{\partial}{\partial \eta}\left[\frac{1}{J}\left(\eta_{x} F+\eta_{y} G\right)\right]+\frac{S}{J y}=0
$$

Finalmente temos:

$$
\frac{\partial \hat{U}}{\partial t}+\frac{\partial \hat{F}}{\partial \xi}+\frac{\partial \hat{G}}{\partial \eta}+\hat{S}=0
$$

definidas por: 


$$
\begin{aligned}
& \hat{U}=\frac{U}{J} \\
& \hat{F}=\frac{1}{J}\left(\xi_{x} F+\xi_{y} G\right) \\
& \hat{G}=\frac{1}{J}\left(\eta_{x} F+\eta_{y} G\right) \\
& \hat{S}=\frac{S}{J y}
\end{aligned}
$$

onde, $\hat{U}$ é o vetor incógnita das variáveis de estado (variáveis de fluxo), $\hat{F}$ e $\hat{G}$ são as funções vetoriais de fluxo e $\hat{S}$ o termo fonte, todas elas nas coordenadas curvilíneas ortogonais. Pode-se observar que as variáveis em Eqs. (3.61) são expressas pelas variáveis $U, F, G$ e o termo fonte $S$, definidos por:

$$
U=\left[\begin{array}{c}
\rho \\
\rho \mathrm{u} \\
\rho \mathrm{v} \\
E
\end{array}\right] \quad F=\left[\begin{array}{c}
\rho \mathrm{u} \\
\rho \mathrm{u}^{2}+p \\
\rho \mathrm{uv} \\
(E+p) \mathrm{u}
\end{array}\right] \quad G=\left[\begin{array}{c}
\rho \mathrm{v} \\
\rho \mathrm{uv} \\
\rho \mathrm{v}^{2}+p \\
(E+p) \mathrm{v}
\end{array}\right] \quad S=\left[\begin{array}{c}
\rho \mathrm{v} \\
\rho \mathrm{uv} \\
\rho \mathrm{v}^{2} \\
(E+p) \mathrm{v}
\end{array}\right]
$$

sendo os mesmos definidos na Eq. (3.30).

Dessa forma temos obtido as equações que governam a dinâmica dos fluidos em coordenadas curvilíneas ortogonais, $(\xi, \eta)$ para um domínio axissimétrico 2D. 


\section{SOLUÇÃO NUMÉRICA: MÉTODO ESTACIONÁRIO}

O fenômeno de evaporação rápida em jatos de líquidos metaestáveis estudado desde o reservatório até sua expansão bifásica é um problema não estacionário. O mesmo fenômeno estudado unicamente no domínio da expansão bifásica é um problema estacionário. No caso 2D, Vieira (1999, 2005) estuda experimentalmente este fenômeno (desde o reservatório até sua expansão bifásica) sob condições de regime permanente. Angelo (2000, 2004) estuda numericamente o fenômeno de evaporação rápida, no domínio da expansão bifásica, sob condições de regime permanente.

Neste capítulo estudaremos numericamente o fenômeno de evaporação rápida no domínio da expansão bifásica sob condições de regime permanente e utilizaremos o método de diferenças finitas com esquema de MacCormack para um modelo estacionário e viscosidade artificial. Para a captura da onda de choque utilizamos uma técnica de varredura de trás para frente e logo interpolamos os pontos onde se produziram esses choques locais. Finalmente refinaremos a malha de forma analítica.

\subsection{Equação Governante}

O fenômeno de evaporação rápida em jatos de líquidos altamente superaquecidos, no domínio da expansão bifásica axissimétrico 2D, é modelado pela equação de conservação para um escoamento em regime permanente que é obtida anulando o termo temporal da Eq. (3.60). Para simplificar as notações das variáveis de fluxo na Eq. (3.61) usaremos $\Phi$ no lugar de $\hat{F}$ e $\Psi$ no lugar de $\hat{G}$, assim temos:

$$
\frac{\partial \Phi}{\partial \xi}+\frac{\partial \Psi}{\partial \eta}+\hat{S}=0
$$

Para o cálculo de alguns resultados nas seções seguintes, é necessário definir a seguinte função de fluxo intermediária:

$$
\Phi^{*}=\Phi^{*}\left(\rho^{*}, \mathrm{u}^{*}, \mathrm{v}^{*}, p^{*}, e^{*}\right)=\frac{1}{J}\left(\xi_{x} F^{*}+\xi_{y} G^{*}\right)
$$


Onde:

$$
F^{*}=\left[\begin{array}{c}
\rho^{*} \mathrm{u}^{*} \\
\rho^{*} \mathrm{u}^{* 2}+p^{*} \\
\rho^{*} \mathrm{u}^{*} \mathrm{v}^{*} \\
\left(E^{*}+p^{*}\right) \mathrm{u}^{*}
\end{array}\right], \quad G^{*}=\left[\begin{array}{c}
\rho^{*} \mathrm{v}^{*} \\
\rho^{*} \mathrm{u}^{*} \mathrm{v}^{*} \\
\rho^{*} \mathrm{v}^{* 2}+p^{*} \\
\left(E^{*}+p^{*}\right) \mathrm{v}^{*}
\end{array}\right]
$$

Na Fig. 4.1 apresentamos uma célula padrão tanto para o domínio físico quanto para o domínio computacional. Dessa forma, a função de fluxo na camada $j+1$ é dada por:

$$
\Phi^{j+1}=\Phi^{j+1}\left(\rho^{j+1}, \mathrm{u}^{j+1}, \mathrm{v}^{j+1}, p^{j+1}, e^{j+1}\right)=\frac{1}{J}\left(\xi_{x} F^{j+1}+\xi_{y} G^{j+1}\right)
$$
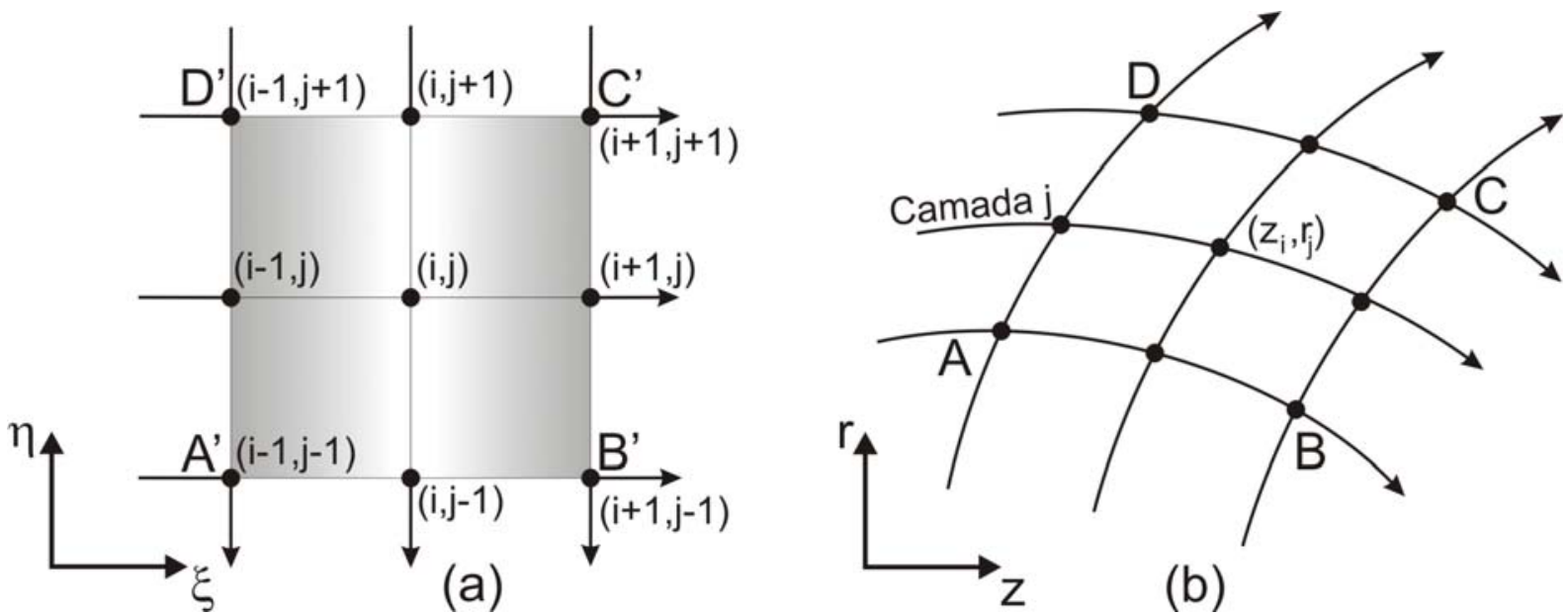

Figura 4.1 Célula padrão da malha. (a) Célula do domínio computacional. (b) Célula do domínio físico

\subsection{Esquema de MacCormack}

O esquema de MacCormack (usado pela primeira vez por MacCormack (1969)) é um método em dois passos e de segunda ordem de precisão. O passo Preditor, usa aproximações de diferenças finitas para trás e o passo Corretor usa aproximações de diferenças finitas para frente. 
Passo Preditor:

$$
\Phi_{i}^{*}=\Phi_{i}^{j}+\Delta \xi\left[\frac{\partial \Phi}{\partial \xi}\right]_{i}^{j}
$$

Onde a derivada parcial é aproximada por diferenças finitas para trás.

$$
\left[\frac{\partial \Phi}{\partial \xi}\right]_{i}^{j}=[\hat{S}]_{i}^{j}-\left[\frac{\partial \Psi}{\partial \eta}\right]_{i}^{j}=[\hat{S}]_{i}^{j}-\left(\frac{\Psi_{i}^{j}-\Psi_{i-1}^{j}}{\Delta \eta}\right)
$$

Passo Corretor:

$$
\Phi_{i}^{j+1}=\frac{1}{2}\left(\Phi_{i}^{j}+\Phi_{i}^{*}+\Delta \xi\left[\frac{\partial \Phi}{\partial \xi}\right]_{i}^{*}\right)
$$

Onde a derivada parcial é aproximada por diferenças finitas para frente.

$$
\left[\frac{\partial \Phi}{\partial \xi}\right]_{i}^{*}=[\hat{S}]_{i}^{*}-\left[\frac{\partial \Psi}{\partial \eta}\right]_{i}^{*}=[\hat{S}]_{i}^{*}-\left(\frac{\Psi_{i+1}^{*}-\Psi_{i}^{*}}{\Delta \eta}\right)
$$

\subsection{Viscosidade Artificial}

O esquema de MacCormack apresenta oscilações (não físicas) na vizinhança de ondas de choque, repercutindo desta maneira na solução numérica. Um caminho para resolver estas oscilações é adicionando um termo de viscosidade artificial, Caramana et al. (1998). Em ambos os passos: Preditor e Corretor, usaremos a definição de viscosidade artificial segundo Anderson (1995). No passo Preditor, a viscosidade artificial é definida por:

$$
\mathfrak{A}_{i}^{j}=C_{x} \frac{\left|p_{i+1}^{j}-2 p_{i}^{j}+p_{i-1}^{j}\right|}{p_{i+1}^{j}+2 p_{i}^{j}+p_{i-1}^{j}}\left[\Phi_{i+1}^{j}-2 \Phi_{i}^{j}+\Phi_{i-1}^{j}\right]
$$

Onde $C_{x}$ é um parâmetro adimensional que controla a influência da viscosidade artificial no escoamento. Assim a Eq. (4.5) resulta em: 


$$
\Phi_{i}^{*}=\Phi_{i}^{j}+\Delta \xi\left[\frac{\partial \Phi}{\partial \xi}\right]_{i}^{j}+\mathfrak{A}_{i}^{j}
$$

No passo Corretor, a viscosidade artificial é definida por:

$$
\mathfrak{A}_{i}^{*}=C_{x} \frac{\left|p_{i+1}^{*}-2 p_{i}^{*}+p_{i-1}^{*}\right|}{p_{i+1}^{*}+2 p_{i}^{*}+p_{i-1}^{*}}\left[\Phi_{i+1}^{*}-2 \Phi_{i}^{*}+\Phi_{i-1}^{*}\right]
$$

Substituindo esta última equação em Eq. (4.7), temos:

$$
\Phi_{i}^{j+1}=\frac{1}{2}\left(\Phi_{i}^{j}+\Phi_{i}^{*}+\Delta \xi\left[\frac{\partial \Phi}{\partial \xi}\right]_{i}^{*}\right)+\mathfrak{A}_{i}^{*}
$$

Conhecida a variável de fluxo $\Phi_{i}^{j+1}$ (avaliada na camada $j+1$ e na posição $i$ ) passamos a decodificar as variáveis primitivas.

\subsection{Decodificação nas Variáveis Primitivas}

A decodificação é o processo de transformar as variáveis de fluxo em variáveis primitivas. Matematicamente significa resolver um sistema algébrico não linear. Para o passo Preditor, o sistema algébrico não linear é obtido das Eqs. (4.2) e (4.10). Assim, temos:

$$
\Phi_{i}^{*}\left(\rho_{i}^{*}, \mathrm{u}_{i}^{*}, \mathrm{v}_{i}^{*}, p_{i}^{*}, e_{i}^{*}\right)=\Phi_{i}^{*}
$$

onde o termo do lado direito é o valor intermediário conhecido. Para o passo Corretor, o sistema algébrico não linear é dado pelas Eqs. (4.4) e (4.12) . Assim temos:

$$
\Phi_{i}^{j+1}\left(\rho_{i}^{j+1}, \mathrm{u}_{i}^{j+1}, \mathrm{v}_{i}^{j+1}, p_{i}^{j+1}, e_{i}^{j+1}\right)=\Phi_{i}^{j+1}
$$

onde o termo do lado direito é o valor da função fluxo recém calculada na camada $j+1$.

Na solução de estes sistemas algébricos não lineares usamos a Tabela de Valores Termodinâmicos da Equação de Lee-Kesler. 


\subsection{Captura da Onda de Choque}

O esquema de MacCormack com viscosidade artificial resolve o problema bifásico e, elimina quase totalmente as oscilações com ajuda de linearizações (alisamentos) das propriedades termodinâmicas na ponta do jato. No entanto, por não utilizar o valor da pressão como condição de contorno na câmara de injeção, ele se expande livremente, convergindo sua solução ao vácuo absoluto (limitado pela geometria do domínio). Por causa desse fato, e outros, o método não captura a onda de choque, porém, aplicados a problemas não estacionários de Leis de conservação 1D, captura a onda de choque como mostrado em Angelo (2004).

Uma técnica de captura da onda de choque foi desenvolvida por Angelo (2004). A técnica consiste numa varredura de trás para frente, isto é, no sentido contrário à expansão bifásica, aplicando-se tentativamente ondas de choques oblíquas "locais” entre os nós de cada linha, que divide a malha. A idéia fundamental para esta técnica é que: “as condições à jusante desta onda de choque localmente oblíqua guarde alguma relação específica com as condições de estagnação pós-onda de choque local”. Esta relação específica é que: a pressão de estagnação à jusante da onda de choque local, $P_{0}^{l}$, seja igual à pressão da câmara de baixa pressão, isto é:

$$
P_{0}^{l}=P_{\infty}
$$

Durante a expansão bifásica, o estado à jusante de uma onda de choque é subsônico, fato que permite usar a hipótese para a pressão de estagnação, que a rigor seria para números de Mach, $M \leq 0,3$ (onde os efeitos de compressibilidade são desprezíveis). Logo pela equação de Bernoulli para fluidos incompressíveis, temos:

$$
\frac{P_{0}^{l}}{\rho_{4}}+\frac{V_{0}^{l 2}}{2}=\frac{P_{4}}{\rho_{4}}+\frac{V_{4}^{2}}{2}
$$

Usando as hipóteses da pressão de estagnação e Eq. (4.15), temos:

$$
\frac{P_{\infty}}{\rho_{4}}=\frac{P_{4}}{\rho_{4}}+\frac{V_{4}^{2}}{2}
$$

Portanto, existirá uma onda de choque oblíqua local quando se cumpra que:

$$
\frac{P_{\infty}}{\rho_{4}} \leq \frac{P_{4}}{\rho_{4}}+\frac{V_{4}^{2}}{2}
$$


Finalmente a onda de choque na região bifásica, será a curva interpolada por todos os pontos que satisfazem a Eq. (4.18).

\subsection{Refinamento da Malha}

Os motivos pelo qual se refinou a malha foram ter uma melhor informação da geometria da onda de choque na proximidade da parede, assim como também, pelos gradientes elevados das propriedades termodinâmicas nas vizinhanças (região bifásica) da ponta do cone evaporativo. Sabe-se que à medida que se refina uma malha, se tem maior informação das variáveis envolvidas. Devido às dificuldades de refinamento do método numérico gerador de malha (ver subseção 3.3.4), impossibilitou ter essas informações. Por conseguinte, se fizeram refinamentos locais na parede (em 2D é a Linha de parede) e na superfície do cone evaporativo (em 2D é a Linha inicial) usando cálculos geométricos sem nenhuma técnica adaptativa.

O refinamento da malha se faz em cinco passos:

1) Geração da malha: se resolve numericamente o problema de Dirichlet de Eqs. (3.19)(3.21), ver Fig. 4.2a.

2) Geração da malha-i: resolve-se o problema de Dirichlet, para uma sub-região do domínio físico, que contem à linha inicial, ver Fig. 4.2b.

3) Pré-malha refinada: acoplamento da malha-i na malha, ou seja, se juntam os nós da malha-i com os nós da malha, ver Fig. 4.2c.

4) Geração da malha-j: resolve-se numericamente o problema de Dirichlet, para uma sub-região do domínio (com os nós da pré-malha refinada), que contem à parede, ver Fig. 4.2d. 
5) Malha refinada: acoplamento da malha-j da pré-malha refinada, ver Fig. 4.2e. Estas malhas mostradas na Fig. 4.2, são ilustrativas, pois, as verdadeiras malhas usadas em nosso trabalho possuem um refinamento muito maior.

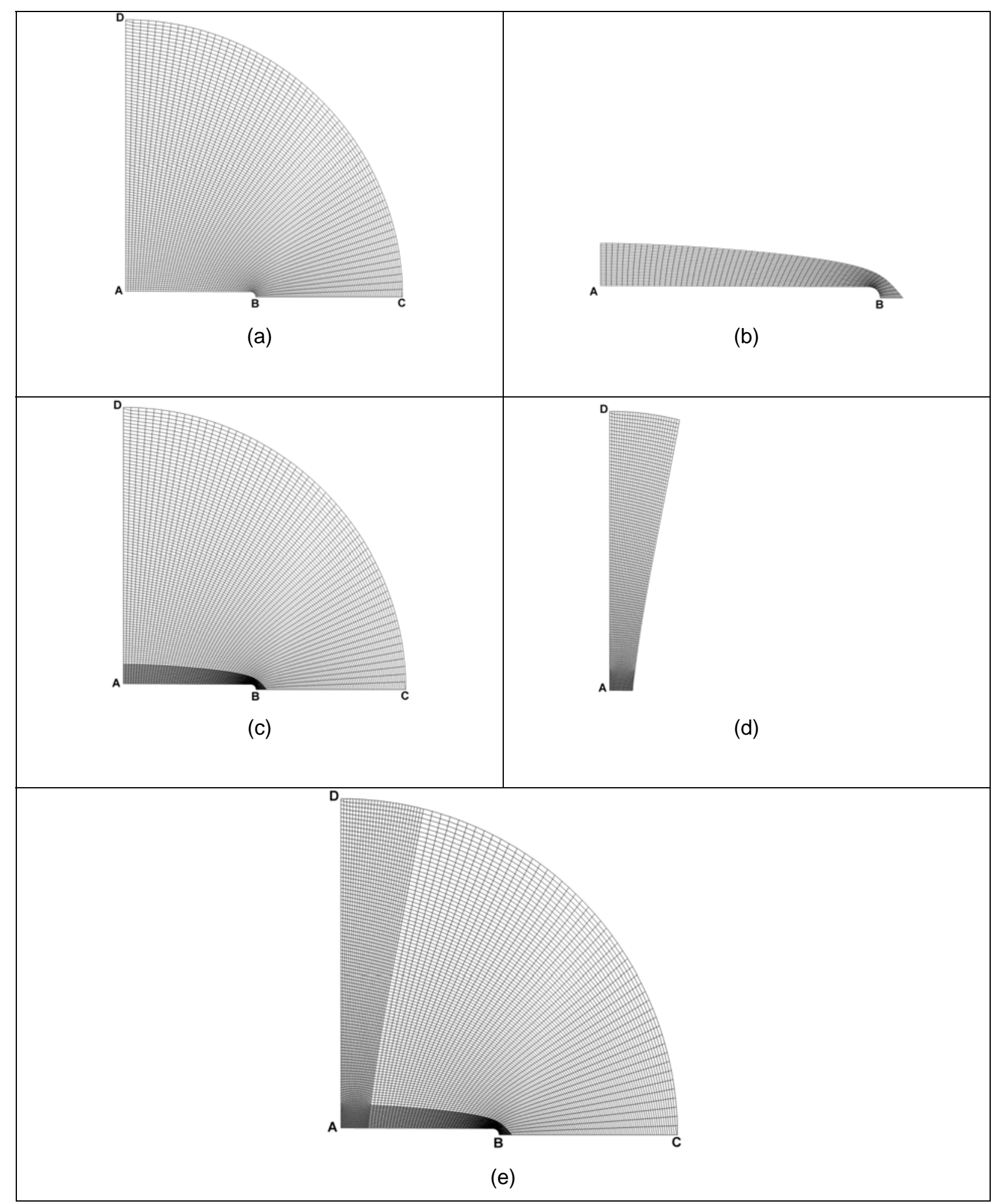

Figura 4.2 (a) Malha. (b) Malha-i. (c) Pré-malha refinada. (d) Malha-j. e) Malha refinada. 
Neste capítulo conseguimos aplicar o método de diferenças finitas com esquema de MacCormak para um modelo estacionário na discretização das equações que governam o fenômeno de evaporação rápida num domínio axissimétrico 2D. Acrescentaram-se os termos de viscosidade artificial nas equações discretizadas para dissipar oscilações não físicas. Aplicou-se a técnica de varredura para a captura da onda de choque e finalmente para obter um formato melhor da onda de choque conseguimos refinar a malha nas proximidades da parede e nas proximidades da Linha inicial. 


\section{SOLUÇÃO NUMÉRICA: MÉTODO NÃO ESTACIONÁRIO}

Os fenômenos modelados matematicamente por sistemas de equações em derivadas parciais independente do tempo são chamados de fenômenos estacionários e os dependentes do tempo de fenômenos não estacionários. Os fenômenos não estacionários podem ser resolvidos numericamente por modelos não estacionários (chamados também de modelos transitórios ou transientes).

Os fenômenos não estacionários da Dinâmica do Fluido Computacional (CFD) modelados matematicamente por Leis de conservação, têm sido estudados numericamente por métodos de alta-resolução. Segundo Shen et al. (2006) afirma que para o caso de Leis de conservação hiperbólicas, os esquemas: “essentially non-oscillatory” (ENO), "Weighted ENO” (WENO), “total variation diminishing” (TVD), Diferenças Finitas Compactas, “Dispersion-Controlled Dissipative” (DCD), por citar alguns, tem sido abordados na captura de descontinuidades.

Neste capítulo estudaremos a decomposição em autovalores das matrizes jacobianas das funções de fluxo, segundo Steger e Warming (1981), das equações de leis de conservação, nas coordenadas cartesianas e nas coordenadas curvilíneas. Também, apresentaremos a formulação por diferenças finitas do esquema DCD para fenômenos não estacionários.

\subsection{Autovalores das Matrizes Jacobianas}

Em 2D sistemas de leis de conservação hiperbólicos que modelam os fluidos invíscidos da dinâmica dos gases tem sido utilizado a teoria de autovalores na aplicação das matrizes jacobianas dos vetores fluxos “Splitting”, veja Steger e Warming (1981). Nesta Secção seguiremos o mesmo procedimento para o cálculo dos autovalores. É importante lembrar que a equação de estado que fecha o sistema hiperbólico é a Equação de Lee-Kesler (veja Apêndice D). 


\subsubsection{Autovalores no sistema de coordenadas cartesianas}

O sistema de leis de conservação em coordenadas cartesianas (veja Eq. (3.29)) é dado por:

$$
\frac{\partial \mathrm{U}}{\partial t}+\frac{\partial F}{\partial x}+\frac{\partial G}{\partial y}+\frac{S}{y}=0
$$

Sabemos que a funções fluxo podem ser expressas como:

$$
\begin{aligned}
& F=A U \\
& G=B U
\end{aligned}
$$

Então temos:

$$
\begin{aligned}
& A=\frac{\partial F}{\partial U} \\
& B=\frac{\partial G}{\partial U}
\end{aligned}
$$

Onde $A$ e $B$ são as matrizes jacobianas de $F$ e $G$, respectivamente. Estas matrizes são da ordem $(4 \times 4)$ e são dadas por:

$$
A=\left(\begin{array}{llll}
\frac{\partial F_{1}}{\partial U_{1}} & \frac{\partial F_{1}}{\partial U_{2}} & \frac{\partial F_{1}}{\partial U_{3}} & \frac{\partial F_{1}}{\partial U_{4}} \\
\frac{\partial F_{2}}{\partial U_{1}} & \frac{\partial F_{2}}{\partial U_{2}} & \frac{\partial F_{2}}{\partial U_{3}} & \frac{\partial F_{2}}{\partial U_{4}} \\
\frac{\partial F_{3}}{\partial U_{1}} & \frac{\partial F_{3}}{\partial U_{2}} & \frac{\partial F_{3}}{\partial U_{3}} & \frac{\partial F_{3}}{\partial U_{4}} \\
\frac{\partial F_{4}}{\partial U_{1}} & \frac{\partial F_{4}}{\partial U_{2}} & \frac{\partial F_{4}}{\partial U_{3}} & \frac{\partial F_{4}}{\partial U_{4}}
\end{array}\right) \quad B=\left(\begin{array}{lllll}
\frac{\partial G_{1}}{\partial U_{1}} & \frac{\partial G_{1}}{\partial U_{2}} & \frac{\partial G_{1}}{\partial U_{3}} & \frac{\partial G_{1}}{\partial U_{4}} \\
\frac{\partial G_{2}}{\partial U_{1}} & \frac{\partial G_{2}}{\partial U_{2}} & \frac{\partial G_{2}}{\partial U_{3}} & \frac{\partial G_{2}}{\partial U_{4}} \\
\frac{\partial G_{3}}{\partial U_{1}} & \frac{\partial G_{3}}{\partial U_{2}} & \frac{\partial G_{3}}{\partial U_{3}} & \frac{\partial G_{3}}{\partial U_{4}} \\
\frac{\partial G_{4}}{\partial U_{1}} & \frac{\partial G_{4}}{\partial U_{2}} & \frac{\partial G_{4}}{\partial U_{3}} & \frac{\partial G_{4}}{\partial U_{4}}
\end{array}\right)
$$

O cálculo destas matrizes jacobinas foi feito no Apêndice C.

Pela teoria de autovalores temos que uma matriz pode ser decomposta da seguinte forma: 


$$
\begin{aligned}
& A=Q \Lambda_{A} Q^{-1} \\
& B=R \Lambda_{B} R^{-1}
\end{aligned}
$$

Onde $Q$ e $R$ são as matrizes dos autovetores correspondentes aos autovalores da matriz $A$ e $B$, respectivamente. Substituindo esta Eq. (5.5) em Eq. (5.2), temos:

$$
\begin{aligned}
& F=Q \Lambda_{A} Q^{-1} U \\
& G=R \Lambda_{B} R^{-1} U
\end{aligned}
$$

Onde $\Lambda_{A}$ e $\Lambda_{B}$ são as matrizes dos autovalores de $A$ e $B$, respectivamente.

Sejam os autovalores de $A$ denotados por $\lambda_{A}$ e os autovalores de $B$ denotado por $\lambda_{B}$. Desenvolveremos algumas propriedades e cálculos da matriz $A$, de forma análoga se fará para a matriz $B$.

Denotemos $\lambda_{A} \equiv \lambda$. Considere a seguinte decomposição para os autovalores da matriz A :

$$
\lambda_{i}=\lambda_{i}^{+}+\lambda_{i}^{-}, \quad i=1, \ldots, 4
$$

Onde

$$
\lambda_{i}^{+}=\frac{\lambda_{i}+\left|\lambda_{i}\right|}{2}, \quad \lambda_{i}^{-}=\frac{\lambda_{i}-\left|\lambda_{i}\right|}{2}
$$

são os autovalores positivos e negativos, respectivamente que satisfaz:

$$
\left\{\begin{array}{ccc}
\lambda_{i}^{+}=\lambda_{i} & \lambda_{i}^{-}=0 & \lambda_{i} \geq 0 \\
\lambda_{i}^{+}=0 & \lambda_{i}^{-}=\lambda_{i} & \lambda_{i}<0
\end{array}\right.
$$

Em forma matricial, temos:

$$
\Lambda_{A}=\Lambda_{A}^{+}+\Lambda_{A}^{-}
$$

Onde: 


$$
\Lambda_{A}^{+}=\left(\begin{array}{cccc}
\lambda_{1}^{+} & & & \\
& \lambda_{2}^{+} & & \\
& & \lambda_{3}^{+} & \\
& & & \lambda_{4}^{+}
\end{array}\right) \quad \Lambda_{B}^{-}=\left(\begin{array}{llll}
\lambda_{1}^{-} & & & \\
& \lambda_{2}^{-} & & \\
& & \lambda_{3}^{-} & \\
& & & \lambda_{4}^{-}
\end{array}\right)
$$

Substituindo Eq. (5.11) em Eq. (5.6), temos:

$$
\begin{aligned}
F & =Q \Lambda_{A} Q^{-1} U \\
& =Q\left(\Lambda_{A}^{+}+\Lambda_{A}^{-}\right) Q^{-1} U \\
& =\left(Q \Lambda_{A}^{+} Q^{-1}+Q \Lambda_{A}^{-} Q^{-1}\right) U \\
& =\left(A^{+}+A^{-}\right) U \\
& =A^{+} U+A^{-} U \\
& =F^{+}+F^{-}
\end{aligned}
$$

Onde:

$$
\begin{gathered}
A^{+}=Q \Lambda_{A}^{+} Q^{-1}, \quad A^{-}=Q \Lambda_{A}^{-} Q^{-1} \\
F^{+}=A^{+} U, \quad F^{-}=A^{-} U
\end{gathered}
$$

e

$$
A=A^{+}+A^{-}
$$

\subsubsection{Autovalores no sistema de coordenadas curvilíneas ortogonais}

Uma vez calculado os autovalores das matrizes jacobianas associadas às funções fluxo $F$ e $G$ podemos calcular os autovalores das matrizes jacobianas associadas às funções fluxos $\hat{F}$ e $\hat{G}$. A seguir apresentamos o cálculo destes autovalores.

O sistema de leis de conservação em coordenadas curvilíneas ortogonais (veja Eq. (3.60)) é dado por:

$$
\frac{\partial \hat{U}}{\partial t}+\frac{\partial \hat{F}}{\partial \xi}+\frac{\partial \hat{G}}{\partial \eta}+\hat{S}=0
$$


onde $\hat{U}, \hat{F}, \hat{G}$, e $\hat{S}$ são a variável de estado, as funções fluxo e o termo fonte nas coordenadas curvilíneas, respectivamente.

$$
\begin{aligned}
& \hat{U}=\frac{U}{J} \\
& \hat{F}=\frac{1}{J}\left(\xi_{x} F+\xi_{y} G\right) \\
& \hat{G}=\frac{1}{J}\left(\eta_{x} F+\eta_{y} G\right) \\
& \hat{S}=\frac{S}{J y}
\end{aligned}
$$

O jacobiano $J$ é dado de acordo à Eq. (3.45) e $\left\{\xi_{x}, \xi_{y}, \eta_{x} \eta_{y}\right\}$ são as métricas definidas na Eq. (3.44). $U, F, G$ e $S$ denotam a variável de estado, os fluxos e a fonte, respectivamente, dados por:

$$
U=\left[\begin{array}{c}
\rho \\
\rho \mathrm{u} \\
\rho \mathrm{v} \\
E
\end{array}\right] \quad F=\left[\begin{array}{c}
\rho \mathrm{u} \\
\rho \mathrm{u}^{2}+p \\
\rho \mathrm{uv} \\
(E+p) \mathrm{u}
\end{array}\right] \quad G=\left[\begin{array}{c}
\rho \mathrm{v} \\
\rho \mathrm{uv} \\
\rho \mathrm{v}^{2}+p \\
(E+p) \mathrm{v}
\end{array}\right] \quad S=\left[\begin{array}{c}
\rho \mathrm{v} \\
\rho \mathrm{uv} \\
\rho \mathrm{v}^{2} \\
(E+p) \mathrm{v}
\end{array}\right]
$$

As variáveis primitivas: $\{\rho, \mathrm{u}, \mathrm{v}, p, e\}$ depende das coordenadas curvilíneas $(\xi, \eta, t)$, elas também podem ser expressas em função das variáveis conservativas. Assim

$$
\rho=U_{1}, \quad \mathrm{u}=\frac{U_{2}}{U_{1}}, \quad \mathrm{v}=\frac{U_{3}}{U_{1}}, \quad e=\frac{U_{4}}{U_{1}}-\frac{1}{2}\left(\frac{U_{2}^{2}+U_{3}^{2}}{U_{1}^{2}}\right), \quad p(\rho, e)
$$

Por outro lado queremos achar as matrizes jacobianas dos vetores fluxos $\hat{F}$ e $\hat{G}$. Fazemos os seguintes cálculos: 


$$
\begin{aligned}
\hat{F} & =\frac{1}{J}\left(\xi_{x} F+\xi_{y} G\right) & \hat{G} & =\frac{1}{J}\left(\eta_{x} F+\eta_{y} G\right) \\
& =\frac{1}{J}\left(\xi_{x} F(U)+\xi_{y} G(U)\right) & & \frac{1}{J}\left(\eta_{x} F(U)+\eta_{y} G(U)\right) \\
& =\frac{1}{J}\left(\xi_{x} A U+\xi_{y} B U\right) & & =\frac{1}{J}\left(\eta_{x} A U+\eta_{y} B U\right) \\
& =\xi_{x} A\left(\frac{U}{J}\right)+\xi_{y} B\left(\frac{U}{J}\right) & & =\eta_{x} A\left(\frac{U}{J}\right)+\eta_{y} B\left(\frac{U}{J}\right) \\
& =\xi_{x} A \hat{U}+\xi_{y} B \hat{U} & & =\eta_{x} A \hat{U}+\eta_{y} B \hat{U} \\
& =\left(\xi_{x} A+\xi_{y} B\right) \hat{U} & & \left(\eta_{x} A+\eta_{y} B\right) \hat{U}
\end{aligned}
$$

Logo:

$$
\begin{aligned}
& \hat{F}=\left(\xi_{x} A+\xi_{y} B\right) \hat{U} \\
& \hat{G}=\left(\eta_{x} A+\eta_{y} B\right) \hat{U}
\end{aligned}
$$

Seja:

$$
\hat{A}=\frac{\partial \hat{F}}{\partial \hat{U}} \quad \hat{B}=\frac{\partial \hat{G}}{\partial \hat{U}}
$$

Definam-se as matrizes jacobianas, nas coordenadas curvilíneas ortogonais $(\xi, \eta)$, da seguinte forma:

$$
\begin{aligned}
& \hat{A}=\xi_{x} A+\xi_{y} B \\
& \hat{B}=\eta_{x} A+\eta_{y} B
\end{aligned}
$$

onde $\hat{A}$ e $\hat{B}$ são as matrizes jacobianas das funções fluxos $\hat{F}$ e $\hat{G}$, respectivamente. Pode-se observar que para o cálculo dos autovalores das matrizes jacobianas $\hat{A}$ e $\hat{B}$ é necessário ter os autovalores das matrizes jacobianas $A$ e $B$. 


\subsection{Esquema "Dispersion-Controlled Dissipative” (DCD)}

O esquema “Dispersion-Controlled Dissipative” (DCD) é um esquema de segunda ordem e pertence à classe dos esquemas "non-oscillatory shock-capturing”. Esta classe é diferente dos esquemas convencionais baseados em dissipação, no qual, os termos dissipativos das equações modificadas não são considerados na construção dos esquemas, podendo assim evitar oscilações não-físicas que ocorrem em simulações de ondas de choques. Este esquema é uma combinação dos esquemas de Lax-Wendroff e Beam-Warming com limitador de fluxo minmod.

Jiang (2004) tem realizado um estudo sistemático sobre o princípio “dispersioncontrolled" desde 1993, e tem publicado as condições de dispersão para esquemas "nonoscillatory shock-capturing” em 1995 (JIANG et al., 1995). O princípio “dispersioncontrolled" visa remover as oscilações não-físicas empregando características da dispersão em vez de adicionar a viscosidade artificial para dissipar as oscilações como fazem os esquemas convencionais. O esquema DCD foi elaborado originalmente para o método de diferenças finitas. No entanto, tem sido estendido para outros métodos como os de volumes finitos e elementos finitos. Além disso, tem se estendido para esquemas de alta ordem.

O esquema DCD, tem sido aplicado em diferentes problemas, por exemplo, ondas de choques toroidais em câmaras cilíndricas (TENG et al., 2005), ondas de choques em combustão de gases (TENG et al., 2007), em processos de ondas dinâmicas na reflexão celular da detonação de cunhas (ZONGMIN et al., 2007).

O esquema DCD foi implementado em um código computacional chamado: DCD-2D v1 ("Dispersion-Controlled Dissipative - Two-Dimensional version 1").

\subsubsection{Formulação por diferenças finitas}

As equações de diferenças finitas para a Eq. (5.17) discretizada espacialmente usando o esquema DCD (JIANG, 1993), são dados em sua forma semi-discreta por:

$$
\left[\frac{\partial \hat{U}}{\partial t}\right]_{i, j}^{n}=-\frac{1}{\Delta \xi}\left(\hat{H}_{i+1 / 2, j}^{n}-\hat{H}_{i-1 / 2, j}^{n}\right)-\frac{1}{\Delta \eta}\left(\hat{P}_{i, j+1 / 2}^{n}-\hat{P}_{i, j-1 / 2}^{n}\right)-\hat{S}_{i, j}^{n}
$$

Com 


$$
\begin{aligned}
\hat{H}_{i+1 / 2, j}^{n} & =\hat{F}_{i+(1 / 2) L, j}^{+}+\hat{F}_{i+(1 / 2) R, j}^{-} \\
\hat{H}_{i-1 / 2, j}^{n} & =\hat{F}_{i-(1 / 2) L, j}^{+}+\hat{F}_{i-(1 / 2) R, j}^{-} \\
\hat{P}_{i, j+1 / 2}^{n} & =\hat{G}_{i, j+(1 / 2) L}^{+}+\hat{G}_{i, j+(1 / 2) R}^{-} \\
\hat{P}_{i, j-1 / 2}^{n} & =\hat{G}_{i, j-(1 / 2) L}^{+}+\hat{G}_{i, j-(1 / 2) R}^{-}
\end{aligned}
$$

Onde os fluxos numéricos são definidos como:

$$
\begin{aligned}
& F_{i+(1 / 2) L, j}^{+}=F_{i, j}^{+}+\frac{1}{2} \Phi_{A}^{+} \operatorname{minmod}\left(\Delta F_{i-1 / 2, j}^{+}, \Delta F_{i+1 / 2, j}^{+}\right) \\
& F_{i+(1 / 2) R, j}^{-}=F_{i+1, j}^{-}-\frac{1}{2} \Phi_{A}^{-} \operatorname{minmod}\left(\Delta F_{i+1 / 2, j}^{-}, \Delta F_{i+3 / 2, j}^{-}\right) \\
& F_{i-(1 / 2) L, j}^{+}=F_{i-1, j}^{+}+\frac{1}{2} \Phi_{A}^{+} \operatorname{minmod}\left(\Delta F_{i-3 / 2, j}^{+}, \Delta F_{i-1 / 2, j}^{+}\right) \\
& F_{i-(1 / 2) R, j}^{-}=F_{i, j}^{-}-\frac{1}{2} \Phi_{A}^{-} \operatorname{minmod}\left(\Delta F_{i-1 / 2, j}^{-}, \Delta F_{i+1 / 2, j}^{-}\right) \\
& G_{i, j+(1 / 2) L}^{+}=G_{i, j}^{+}+\frac{1}{2} \Phi_{B}^{+} \operatorname{minmod}\left(\Delta G_{i, j-1 / 2}^{+}, \Delta G_{i, j+1 / 2}^{+}\right) \\
& G_{i, j+(1 / 2) R}^{-}=G_{i, j+1}^{-}-\frac{1}{2} \Phi_{B}^{-} \operatorname{minmod}\left(\Delta G_{i, j+1 / 2}^{-}, \Delta G_{i, j+3 / 2}^{-}\right) \\
& G_{i, j-(1 / 2) L}^{+}=G_{i, j-1}^{+}+\frac{1}{2} \Phi_{B}^{+} \operatorname{minmod}\left(\Delta G_{i, j-3 / 2}^{+}, \Delta G_{i, j-1 / 2}^{+}\right) \\
& G_{i, j-(1 / 2) R}^{-}=G_{i, j}^{-}-\frac{1}{2} \Phi_{B}^{-} \operatorname{minmod}\left(\Delta G_{i, j-1 / 2}^{-}, \Delta G_{i, j+1 / 2}^{-}\right)
\end{aligned}
$$

onde,

$$
\begin{array}{ll}
\Delta F_{i-3 / 2, j}^{+}=F_{i-1, j}^{+}-F_{i-2, j}^{+} & \Delta G_{i, j-3 / 2}^{+}=G_{i, j-1}^{+}-G_{i, j-2}^{+} \\
\Delta F_{i-1 / 2, j}^{+}=F_{i, j}^{+}-F_{i-1, j}^{+} & \Delta G_{i, j-1 / 2}^{+}=G_{i, j}^{+}-G_{i, j-1}^{+} \\
\Delta F_{i+1 / 2, j}^{+}=F_{i+1, j}^{+}-F_{i, j}^{+} & \Delta G_{i, j+1 / 2}^{+}=G_{i, j+1}^{+}-G_{i, j}^{+} \\
\Delta F_{i-1 / 2, j}^{-}=F_{i, j}^{-}-F_{i-1, j}^{-} & \Delta G_{i, j-1 / 2}^{-}=G_{i, j}^{-}-G_{i, j-1}^{-} \\
\Delta F_{i+1 / 2, j}^{-}=F_{i+1, j}^{-}-F_{i, j}^{-} & \Delta G_{i, j+1 / 2}^{-}=G_{i, j+1}^{-}-G_{i, j}^{-} \\
\Delta F_{i+3 / 2, j}^{-}=F_{i+2, j}^{-}-F_{i+1, j}^{-} & \Delta G_{i, j+3 / 2}^{-}=G_{i, j+2}^{-}-G_{i, j+1}^{-}
\end{array}
$$


e segue a célula computacional, Fig. 5.1 para acompanhar os índices usados nas funções de fluxos numéricos no nó $(i, j)$.

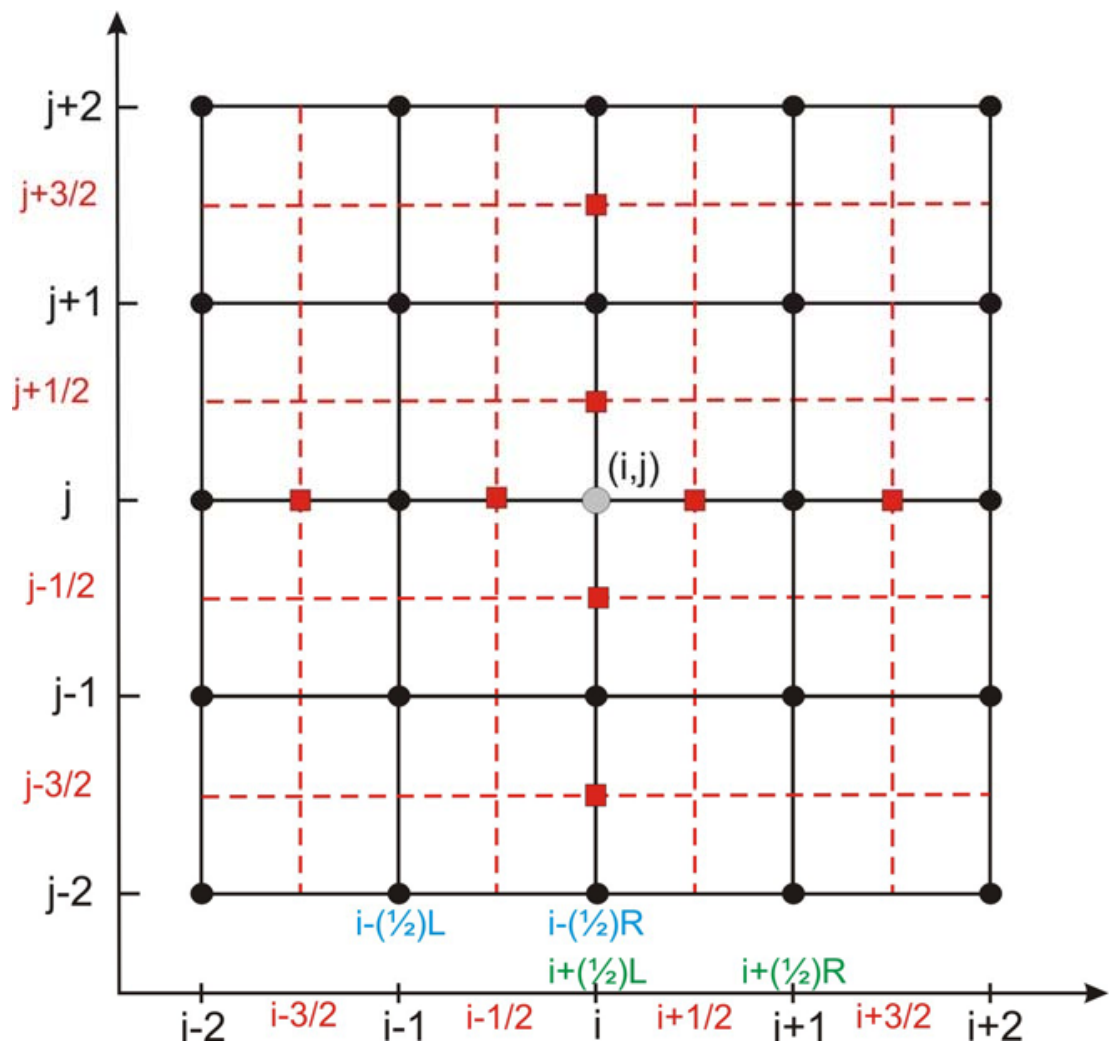

Figura 5.1 Célula computacional para determinar a variação dos fluxos numéricos.

Definam-se as seguintes matrizes:

$$
\begin{aligned}
& \Phi_{A}^{ \pm}=I \mp \beta \Lambda_{A}^{ \pm} \\
& \Phi_{B}^{ \pm}=I \mp \beta \Lambda_{B}^{ \pm}
\end{aligned}
$$

Onde $I$ é a matriz identidade e $\beta$ é um parâmetro definido por:

$$
\beta=\frac{\Delta t}{\Delta \xi}
$$

onde $\Delta t$ é o incremento temporal e $\Delta \xi$ o comprimento espacial.

O limitador de fluxo é a função minmod definida por: 


$$
\operatorname{minmod}(x, y)= \begin{cases}\operatorname{sgn}(x) \min \{|x|,|y|\}, & \operatorname{sgn}(x)=\operatorname{sgn}(y) \\ 0 \quad & \operatorname{sgn}(x) \neq \operatorname{sgn}(y)\end{cases}
$$

onde a função “sgn” é definida por:

$$
\operatorname{sgn}(x)=\left\{\begin{array}{ccc}
1 & \text { se } & x>0 \\
0 & \text { se } & x=0 \\
-1 & \text { se } & x<0
\end{array}\right.
$$

\subsubsection{Discretização temporal}

Conhecidos os dados iniciais das grandezas no instante inicial $t_{0}$, fenômenos não estacionários exigem na solução de um sistema de equações em derivadas parciais ou ordinárias intervalos sucessivos de tempo $\Delta t$ até o instante final $t_{f}$, ou seja:

$$
t_{0}, t_{0}+\Delta t,+t_{0}+2 \Delta t+\ldots+t_{f}
$$

Definamos em primeiro lugar a norma euclidiana de uma matriz $A=\left(a_{i, j}\right)_{N \times M}$ por:

$$
\|A\|=\sqrt{\sum_{i=1}^{N} \sum_{j=1}^{M} a_{i j}^{2}}
$$

onde $N, M$ são as dimensões da matriz. Observe que o número de nós que contem o domínio computacional é $(N \times M)$, incluindo os nós do contorno.

Na implementação do código computacional $D C D-2 D v 1$, o tamanho de passo no tempo $\Delta t$, segundo Peyret e Taylor (1983), referente ao domínio computacional, é dado por:

$$
\Delta t=\frac{C_{0}}{\frac{\|\mathrm{u}\|}{d \xi}+\frac{\|\mathrm{v}\|}{d \eta}+\|c\| \sqrt{\frac{1}{d \xi^{2}}+\frac{1}{d \eta^{2}}}}
$$

onde $C_{0}$ é uma constante positiva e toma um valor fixo, isto é: $C_{0}=5.6$. $\{d \xi, d \eta\}$ são as dimensões dos elementos da malha computacional. $\{\|u\|,\|v\|\}$ são as normas dos vetores 
velocidade $\mathrm{u}$ e $\mathrm{v}$, respectivamente; avaliadas em todos os nós do domínio computacional, no caso de u, temos:

$$
\|\mathbf{u}\|=\sqrt{\sum_{i=1}^{N} \sum_{j=1}^{M} u_{i j}^{2}}
$$

onde $u=\left(u_{i j}\right)_{N \times M}$, e $\|c\|$ é a norma da velocidade do som.

Na discretização temporal foi usado o método de Runge-Kutta de segunda ordem. Seja:

$$
U_{t}=L\left(U_{i, j}\right)
$$

a forma discreta da Eq. (5.17), onde $L$ é o operador da discretização espacial para o termo $\left\{-\frac{\partial \hat{F}}{\partial \xi}-\frac{\partial \hat{G}}{\partial \eta}-\hat{S}\right\}$. $U_{i, j}$ é a aproximação discreta de $U$ em $\xi=i \Delta \xi$ e $\eta=j \Delta \eta$. O método de Runge-Kutta de segunda ordem toma a forma:

$$
\left\{\begin{array}{rlc}
k_{1} & = & L\left(U^{n}\right) \\
k_{2} & = & L\left(U^{n}+\frac{\Delta t}{2} k_{1}\right) \\
U^{n+1} & = & U^{n}+\frac{\Delta t}{6}\left[k_{1}+2 k_{2}\right]
\end{array}\right.
$$

\subsubsection{Domínio computacional}

\section{- Classificação dos nós}

No domínio computacional podemos caracterizar diferentes grupos de nós, essenciais quando aproximamos os fluxos numéricos. Na Fig. 5.2 os pontos externos representam os “nós fantasmas”, os pontos do meio (círculos maiores) representam os nós do contorno e os pontos interiores (quadrados) são os nós interiores. O domínio computacional $\Gamma^{\prime}$ é definido por: $\Gamma^{\prime}=\bigcup_{i=1}^{4} \Gamma_{i}^{\prime}$ (veja Eq. 3.10) e tem dimensão $(N+2) \times(M+2)$. 


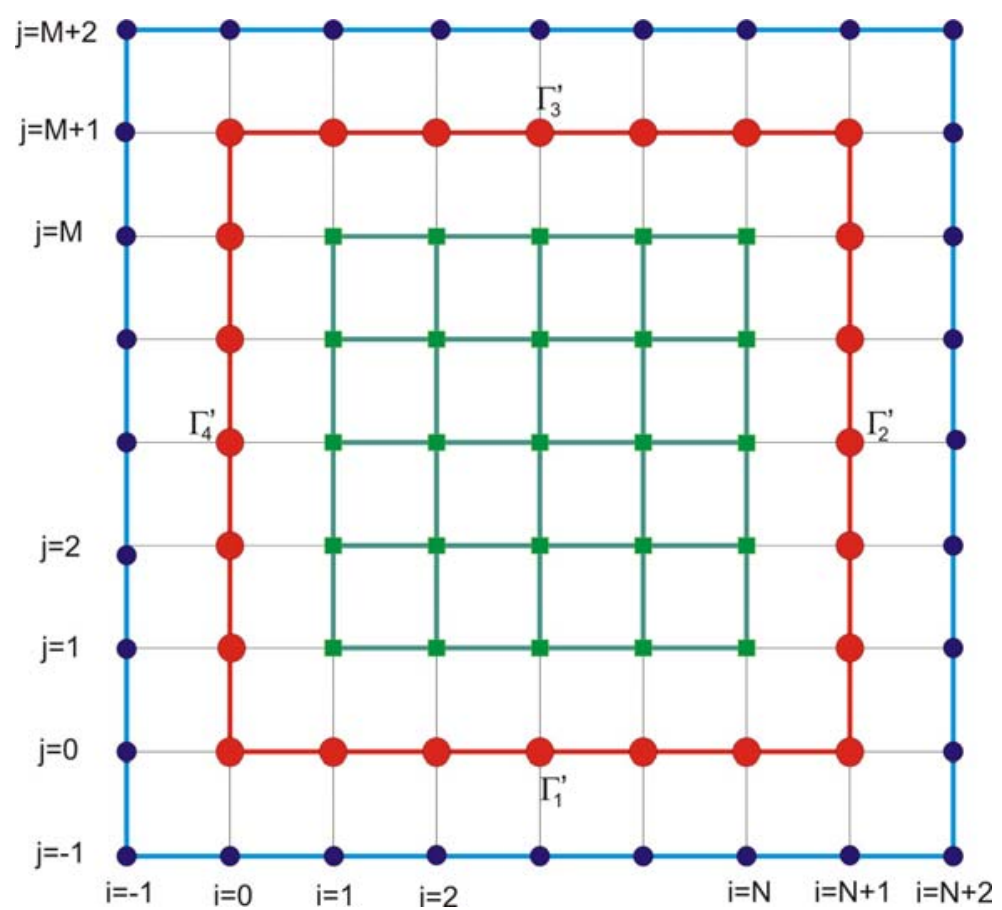

Figura 5.2 Nós do domínio computacional.

\section{- $\quad$ Condições de contorno numéricas}

As condições de contorno numéricas não carregam nenhum significado físico e são extrapoladas para nós vizinhos. A seguir temos algumas delas:

\section{Extrapolação de ordem zero}

$$
\begin{array}{ll}
u_{i, j}=u_{i-1, j} & u_{i, j}=u_{i+1, j} \\
u_{i, j}=u_{i, j-1} & u_{i, j}=u_{i, j+1}
\end{array}
$$

\section{Extrapolação de ordem 1}

$$
\begin{array}{ll}
u_{i, j}=2 u_{i-1, j}-u_{i-2, j} & u_{i, j}=2 u_{i+1, j}-u_{i+2, j} \\
u_{i, j}=2 u_{i, j-1}-u_{i, j-2} & u_{i, j}=2 u_{i, j+1}-u_{i, j+2}
\end{array}
$$

Portanto, neste capítulo se conseguiu obter os autovalores das matrizes jacobianas. Apresentou-se o esquema "shock-capturing” DCD que discretiza o sistema de leis de conservação hiperbólicas. A discretização temporal pelo método de Runge-Kutta de segunda ordem foi estudado, assim como também os nós do domínio computacional e as condições de contorno numéricas. 


\section{RESULTADOS NUMÉRICOS: CASO ESTACIONÁRIO}

Neste capítulo apresentaremos os resultados numéricos processados pelo código ShoWPhasT-2D v2, partindo das condições de contorno para a Linha inicial. Obtendo diferentes geometrias de onda de choque ao variar a pressão na câmara de injeção (pressão ao longe), como também seu formato ao refinar a malha. Estes resultados numéricos são comparados com resultados experimentais de Vieira (2005) e (VIEIRA; SIMÕESMOREIRA, 2007) e com resultados feito pelo código ShoWPhasT-2D v1 de Angelo (2004) e (ANGELO; SIMÕES-MOREIRA, 2007), realizados no Laboratório SISEA.

\subsection{Condição de Contorno na Linha inicial}

Para resolver numericamente a expansão bifásica do fenômeno de evaporação rápida (“flashing”) de jatos de líquidos superaquecidos, necessitamos de condições de contorno na Linha inicial (Eq. (3.1)). Estas condições de contorno são dadas na Tabela 6.1 para um total de 20 testes. Onde as duas primeiras colunas são a pressão e a temperatura de reservatório, tomadas dos ensaios de Vieira (2005). Os subscritos 2 que aparecem nesta tabela estão indicando a solução de Chapman-Jouguet que representam as condições de contorno na Linha inicial. Os dados experimentais obtidos para um bocal de raio $R_{b}=0,150$ [mm], são: $\left\{\dot{m}, C_{D}, L_{E}\right\}$ e fornecidos, também, por Vieira (2005). É importante frisar que estas condições de contorno foram obtidas numericamente, porém não usamos o mesmo raio do bocal do experimento, e $\operatorname{sim} R_{b}=0,153$ [mm], devido ao fato da pressão metaestável dada pela Eq. (2.7) assumir valores negativos. Estes valores negativos se devem à existência de uma margem de erro de $\pm 2 \%$ da determinação da vazão em massa (VIEIRA; SIMÕESMOREIRA, 2007). No caso de considerar o mesmo $R_{b}$ teríamos que diminuir a vazão em massa nessa margem de erro para cada teste. 
Tabela 6.1 Estados Termodinâmicos da Pré-expansão Bifásica e Pós-onda de Evaporação para $R_{b}=0,1530[\mathrm{~mm}]$ e $\left\{P_{0}, T_{0}\right\}$ conforme a (Vieira, 2005).

\begin{tabular}{|c|c|c|c|c|c|c|c|c|c|c|c|c|c|c|}
\hline $\begin{array}{c}P_{0} \\
{[\mathrm{kPa}]}\end{array}$ & $\begin{array}{c}T_{0} \\
{\left[{ }^{\circ} \mathrm{C}\right]}\end{array}$ & $\begin{array}{c}\dot{m} \\
{[\mathrm{~g} / \mathrm{s}]}\end{array}$ & $\begin{array}{c}C_{D} \\
{[]}\end{array}$ & $\begin{array}{c}v_{1} \\
{\left[\mathrm{~m}^{3} / \mathrm{kg}\right]}\end{array}$ & $\begin{array}{c}P_{1} \\
{[\mathrm{kPa}]}\end{array}$ & $\begin{array}{c}P_{2} \\
{[\mathrm{kPa}]}\end{array}$ & $\begin{array}{c}T_{2} \\
{\left[{ }^{\circ} \mathrm{C}\right]}\end{array}$ & $\begin{array}{r}X_{2} \\
{[]}\end{array}$ & $\begin{array}{c}v_{2} \\
{\left[\mathrm{~m}^{3} / \mathrm{kg}\right]}\end{array}$ & $\begin{array}{c}W_{2} \\
{[\mathrm{~m} / \mathrm{s}]}\end{array}$ & $\begin{array}{c}\beta \\
{\left[{ }^{\circ}\right]}\end{array}$ & $\begin{array}{c}\theta \\
{\left[{ }^{\circ}\right]}\end{array}$ & $\begin{array}{c}L_{E} \\
{[\mathrm{~mm}]}\end{array}$ & $\begin{array}{c}R_{n b} \\
{[\mathrm{~mm}]}\end{array}$ \\
\hline 122,1 & 56,4 & 0,8447 & 0,9124 & 0,0014936 & 3,7505 & 2,015 & 2,4817 & 0,35658 & 3,53975 & 78,4 & 0,1105 & 77,55 & 7,2 & 0,8617 \\
\hline 122,7 & 75,2 & 0,8340 & 0,9165 & 0,0015358 & 5,1278 & 2,689 & 7,5863 & 0,46100 & 3,49036 & 92,3 & 0,1336 & 79,18 & 4,8 & 1,0732 \\
\hline 123,5 & 94,8 & 0,8022 & 0,9195 & 0,0015834 & 12,0842 & 6,369 & 24,2918 & 0,51084 & 1,72455 & 99,3 & 0,3024 & 79,83 & 3,2 & 0,7230 \\
\hline 249,0 & 56,9 & 1,2103 & 0,9215 & 0,0014947 & 10,6345 & 6,306 & 24,0858 & 0,23081 & 0,78724 & 58,4 & 0,2583 & 66,90 & 8,0 & 0,3465 \\
\hline 251,8 & 76,6 & 1,1872 & 0,9250 & 0,0015390 & 17,4199 & 9,983 & 33,9830 & 0,31276 & 0,69423 & 71,9 & 0,3676 & 70,57 & 6,1 & 0,3233 \\
\hline 250,6 & 94,7 & 1,1210 & 0,9269 & 0,0015831 & 36,5273 & 21,380 & 52,2525 & 0,33266 & 0,36292 & 74,3 & 0,7696 & 71,24 & 5,2 & 0,2051 \\
\hline 249,6 & 120,2 & 0,9456 & 0,9285 & 0,0016519 & 91,2091 & 55,294 & 79,0715 & 0,35503 & 0,15973 & 76,1 & 2,1235 & 72,29 & 3,1 & 0,1608 \\
\hline 257,1 & 130,9 & 0,8102 & 0,9258 & 0,0016836 & 137,8926 & 86,706 & 93,6925 & 0,33878 & 0,10024 & 72,2 & 3,7486 & 71,87 & 2,6 & 0,1733 \\
\hline 501,6 & 56,6 & 1,7455 & 0,9291 & 0,0014940 & 14,1018 & 8,852 & 31,3132 & 0,18178 & 0,45207 & 48,8 & 0,2606 & 53,74 & 12,5 & 0,2357 \\
\hline 497,6 & 76,9 & 1,6993 & 0,9319 & 0,0015397 & 24,2793 & 14,638 & 42,8503 & 0,25655 & 0,39876 & 62,1 & 0,3862 & 59,81 & 9,6 & 0,2148 \\
\hline 503,4 & 95,4 & 1,6528 & 0,9339 & 0,0015849 & 44,4770 & 26,863 & 58,2506 & 0,29653 & 0,26169 & 68,1 & 0,6634 & 61,73 & 5,7 & 0,2129 \\
\hline 503,3 & 120,6 & 1,4822 & 0,9354 & 0,0016530 & 119,5908 & 77,961 & 90,1101 & 0,27299 & 0,08951 & 61,6 & 1,9568 & 59,65 & 3,9 & 0,1676 \\
\hline 500,0 & 131,0 & 1,3757 & 0,9354 & 0,0016839 & 163,2676 & 108,606 & 101,5594 & 0,27552 & 0,06636 & 61,0 & 2,8166 & 59,90 & 3,6 & 0,1581 \\
\hline 502,1 & 162,1 & 0,9286 & 0,9328 & 0,0017896 & 338,1384 & 229,639 & 130,7932 & 0,33106 & 0,03939 & 66,9 & 7,7301 & 63,76 & 2,5 & 0,1568 \\
\hline 751,6 & 56,1 & 2,1471 & 0,9327 & 0,0014929 & 20,1864 & 14,637 & 42,8479 & 0,09889 & 0,15463 & 29,4 & 0,3731 & 33,63 & 14,8 & 0,1701 \\
\hline 752,5 & 77,0 & 2,0910 & 0,9354 & 0,0015400 & 41,0683 & 29,188 & 60,4969 & 0,13157 & 0,10832 & 36,1 & 0,6716 & 38,83 & 11,3 & 0,1689 \\
\hline 748,4 & 95,3 & 2,0112 & 0,9371 & 0,0015846 & 73,6089 & 52,848 & 77,6743 & 0,14998 & 0,07128 & 38,9 & 1,1434 & 40,78 & 7,6 & 0,1701 \\
\hline 753,2 & 121,1 & 1,8833 & 0,9390 & 0,0016545 & 137,9207 & 94,809 & 96,7659 & 0,22311 & 0,06127 & 52,1 & 1,9029 & 48,99 & 5,0 & 0,1866 \\
\hline 749,6 & 131,9 & 1,7599 & 0,9392 & 0,0016867 & 202,0721 & 149,926 & 113,5344 & 0,17979 & 0,03265 & 42,4 & 3,1107 & 43,34 & 4,4 & 0,1620 \\
\hline 749,2 & 161,8 & 1,3746 & 0,9389 & 0,0017885 & 394,7948 & 298,304 & 142,2777 & 0,21670 & 0,02075 & 46,8 & 6,9305 & 47,73 & 2,3 & 0,1570 \\
\hline
\end{tabular}

\subsection{Variação da Pressão na Câmara de Injeção}

Num determinado teste computacional com dados de entrada $\left\{P_{0}, T_{0}\right\}$ consegue-se uma única onda de choque para cada pressão na câmara de injeção, $P_{\infty}$. A onda de choque é caracterizada por sua geometria, onde seu comprimento radial (ou vertical) é $2 r_{1}$, sendo que $r_{1}$ é o comprimento radial a partir da Linha de simetria até o ponto onde $r$ é máximo na onda de choque. Seu comprimento axial (ou horizontal) é $r_{2}$, que se inicia a partir da seção de saída do bocal até a interseção da onda de choque com o eixo de simetria. Se mantivermos fixos os dados de entrada $\left\{P_{0}, T_{0}\right\}$ e começarmos a variar, por exemplo, aumentando a pressão da câmara de injeção, $\left\{P_{\infty}\right\}$, observamos que a geometria da onda de choque varia, com diminuição tanto no comprimento radial $r_{1}$, quanto no comprimento axial $r_{2}$. E a variação é proporcionalmente maior na direção radial do que na longitudinal. Para validar estes resultados foi considerado um teste da Tabela 6.1, localizado na linha dez da tabela fornecendo os seguintes dados: $\left\{P_{0}=497,6[\mathrm{kPa}] ; T_{0}=76,9\left[{ }^{\circ} \mathrm{C}\right]\right\}$ com a pressão da câmara de injeção variando e assumindo os seguintes valores:

$$
P_{\infty}[\mathrm{Pa}]=\{100 ; 120 ; 150 ; 200 ; 250 ; 300 ; 400 ; 600\},
$$


assim como mostra a Fig. 6.1. É importante indicar que as unidades dos eixos $z$ e $r$ estão expressados em metros.
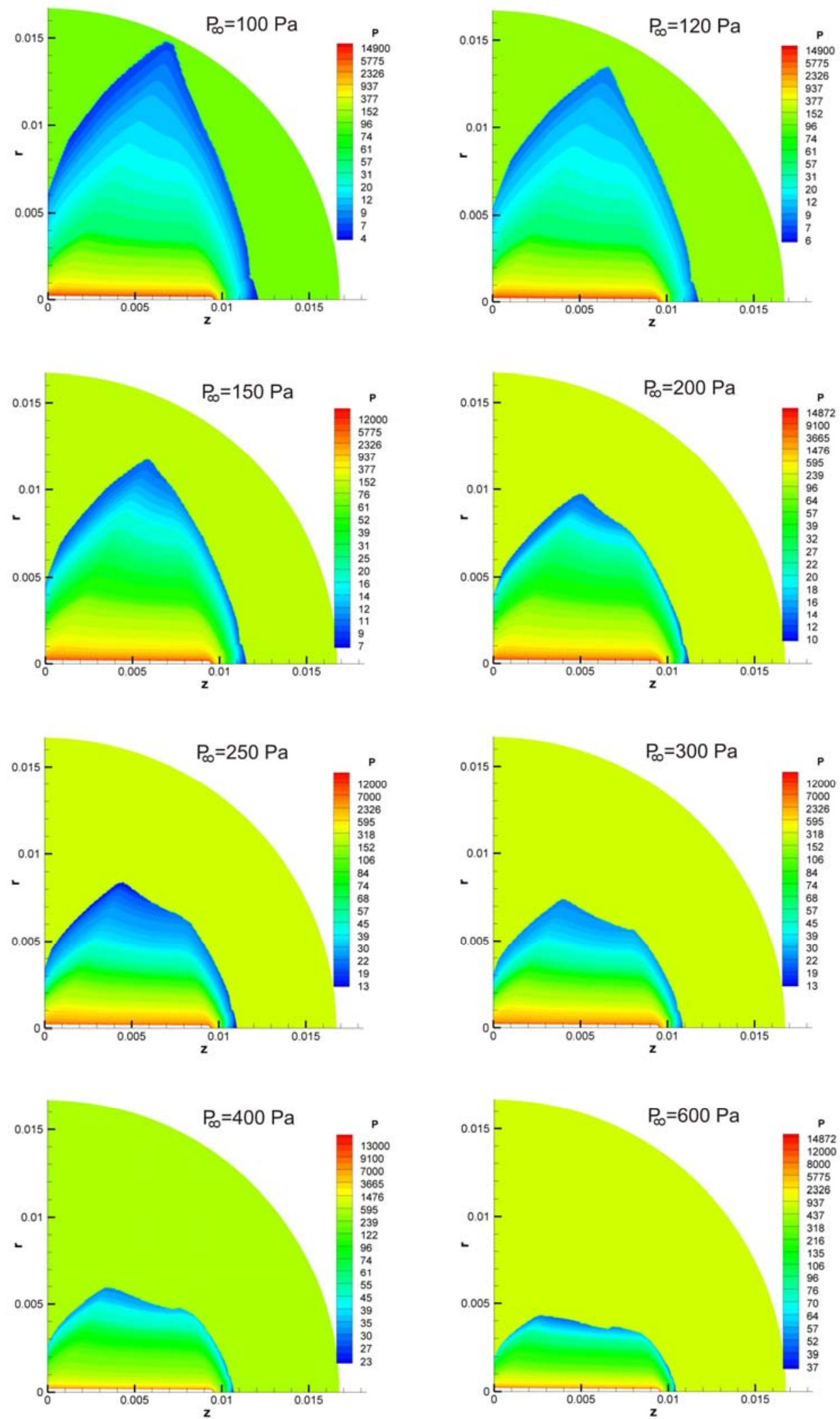

Figura 6.1 Ondas de choques variando a pressão na câmara de injeção. 


\subsection{Refinamento na Malha}

Por meio do refinamento da malha ganhamos maior precisão no formato da onda de choque. Isto ocorre tanto na parede (onde aparece uma onda de choque refletindo-se), quanto no interior do domínio da expansão bifásica, ao procedermos a captura da onda de choque. Para validar nosso resultado consideremos o teste da Tabela 6.1 posicionada na linha doze fornecendo os seguintes dados:

$$
\left\{P_{0}=503,3[\mathrm{kPa}] ; T_{0}=120,6\left[{ }^{\circ} \mathrm{C}\right] ; P_{\infty}=210[\mathrm{~Pa}]\right\} .
$$

Para a malha sem refinamento usamos 24750 nós e para a malha com refinamento usamos 44252 nós, representando um 78,8\% nós a mais, os resultados foram esboçados na Fig. 6.2. As dimensões da onda de choque para o caso sem refinamento são: $r_{1}=9,3$ [mm] e $r_{2}=4,3[\mathrm{~mm}]$ e para o caso com refinamento são: $r_{1}=9,5[\mathrm{~mm}]$ e $r_{2}=4,4[\mathrm{~mm}]$. Para o caso experimental temos: $r_{1}=12,9[\mathrm{~mm}]$ e $r_{2}=6,8[\mathrm{~mm}]$. Pode-se dizer que o caso com refinamento aproxima melhor as dimensões da onda de choque em relação aos dados experimentais. A onda de choque obtida experimentalmente para o mesmo teste (Serie RUN 2, ensaio 5393m10b, imagem 2), segundo Vieira (2005) é apresentado na Fig. 6.3a e uma imagem realçada da onda de choque é mostrada na Fig. 6.3b.

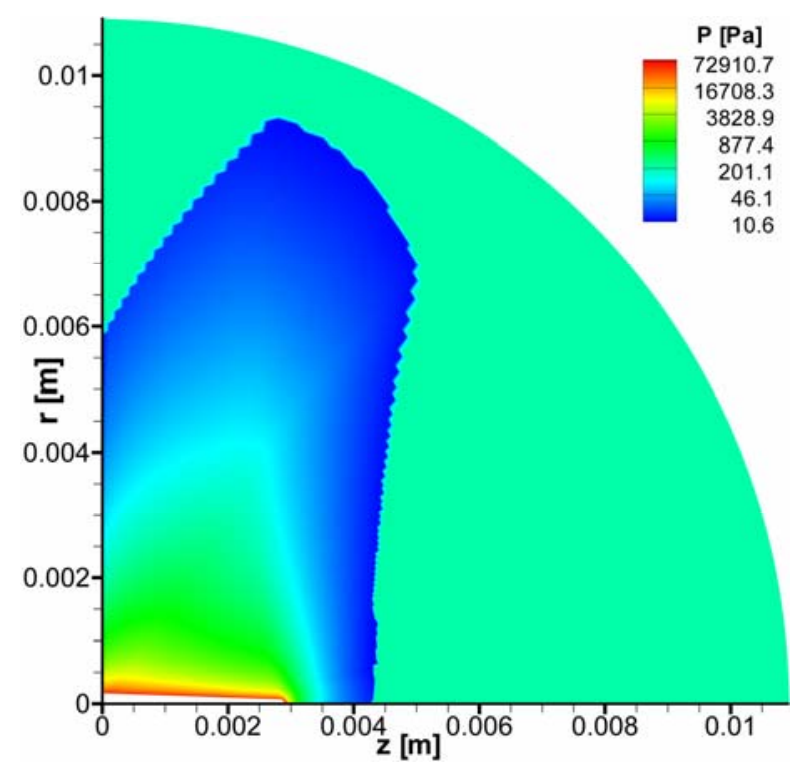

(a)

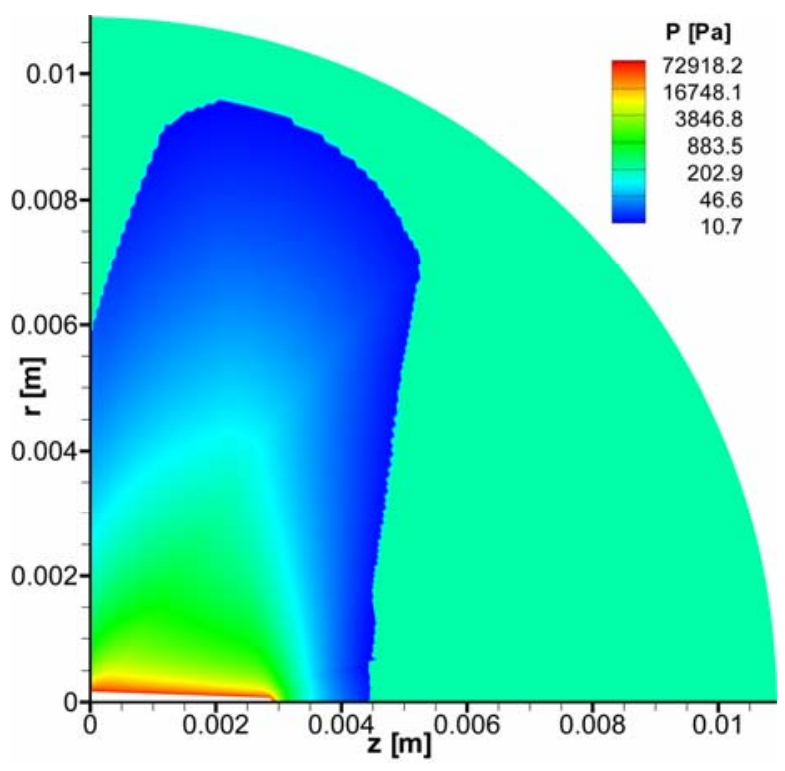

(b)

Figura 6.2 Onda de choque obtida numericamente. a) Sem refinamento. b) Com refinamento. 


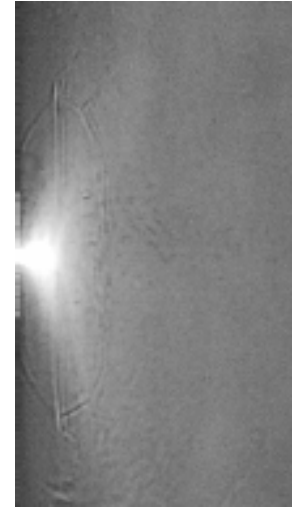

(a)

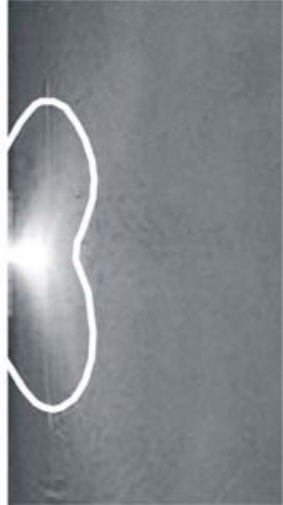

(b)

Figura 6.3 Onda de choque obtida experimentalmente (Serie RUN 2, ensaio 5393m10b, imagem 2), segundo Vieira (2005). a) Foto original. b) Imagem realçada.

\subsection{Comparação com Resultados Experimentais}

Soluções numéricas executadas pelo código ShoWPhasT-2D v2 são comparados com resultados experimentais de Vieira (2005) para um total de vinte testes, com condições de reservatório dados na Tabela 6.1. As comparações foram feitas para as dimensões da onda de choque em dois tipos de malhas: refinadas e não refinadas. E finalmente apresentamos a distribuição de pressões, temperaturas, título e número Mach contendo a onda de choque.

\subsubsection{Variação das condições de reservatório}

Para validar nosso código computacional ShoWPhasT-2D v2 fizemos vinte testes numéricos com diferentes condições de reservatórios, conforme ensaios experimentais realizados no Laboratório SISEA. Comparamos as geometrias da onda de choque fornecidas por nossos resultados numéricos com os resultados experimentais de Vieira (2005) para um bocal de raio $R_{b}=0,30[\mathrm{~mm}]$. Estes resultados são mostrados na Tabela 6.2. No que concerne os resultados numéricos, as dimensões da onda de choque são analisadas em dois grupos de malhas, um sem refinamento e a outra com refinamento. 
Tabela 6.2 Resultados Experimentais e Numéricos: Variação das condições de reservatório.

\begin{tabular}{|c|c|c|c|c|c|c|c|c|c|c|c|}
\hline \multirow{2}{*}{\multicolumn{2}{|c|}{$\begin{array}{l}\text { Condições de } \\
\text { Reservatório }\end{array}$}} & \multirow{2}{*}{\multicolumn{3}{|c|}{ Dados Experimentais }} & \multirow{2}{*}{\multicolumn{2}{|c|}{$\begin{array}{c}\text { Resultados } \\
\text { Experimentais }\end{array}$}} & \multicolumn{5}{|c|}{ Resultados Numéricos } \\
\hline & & & & & & & & \multicolumn{2}{|c|}{$\begin{array}{c}\text { Sem } \\
\text { Refinamento }\end{array}$} & \multicolumn{2}{|c|}{$\begin{array}{c}\text { Com } \\
\text { Refinamento }\end{array}$} \\
\hline$P_{0}[\mathrm{kPa}]$ & $T_{0}\left[{ }^{\circ} \mathrm{C}\right]$ & Experimento & Series & $P_{\infty}[\mathrm{Pa}]$ & $r_{1}$ & $r_{2}$ & Teste & $r_{1}$ & $r_{2}$ & $r_{1}$ & $r_{2}$ \\
\hline 122,1 & 56,4 & 1329050 & Run3 & 500 & 4,0 & 8,1 & 1 & 4,8 & 8,9 & 4,9 & 8,9 \\
\hline 122,7 & 75,2 & 1349500 & Run3 & 140 & 9,7 & 8,4 & 2 & 11,0 & 9,9 & 10,8 & 10,4 \\
\hline 123,5 & 94,8 & 1368500 & Run3 & 150 & 14,7 & 8,8 & 3 & 10,4 & 8,7 & 10,2 & 8,8 \\
\hline 249,0 & 56,9 & 2329500 & Run2 & 80 & 13,8 & 12,4 & 4 & 13,3 & 11,4 & 14,1 & 11,5 \\
\hline 251,8 & 76,6 & 2349500b & Run1 & 180 & 7,7 & 8,4 & 5 & 10,0 & 8,6 & 10,3 & 8,7 \\
\hline 250,6 & 94,7 & 2368500 & Run1 & 200 & 15,7 & 9,9 & 6 & 9,2 & 6,9 & 9,4 & 7,0 \\
\hline 249,6 & 120,2 & 2393500 & Run1 & 250 & 10,1 & 5,9 & 7 & 8,5 & 3,7 & 7,5 & 3,8 \\
\hline 257,1 & 130,9 & 2404500b & Run1 & 480 & 7,5 & 4,7 & 8 & 4,6 & 2,5 & 4,6 & 2,5 \\
\hline 501,6 & 56,6 & 5329100 & Run3 & 200 & 13,8 & 18,2 & 9 & 7,2 & 14,1 & 7,9 & 14,1 \\
\hline 497,6 & 76,9 & 5349500 & Run3 & 110 & 13,5 & 11,9 & 10 & 12,6 & 11,8 & 14,1 & 11,9 \\
\hline 503,4 & 95,4 & 5368100 & Run2 & 960 & 5,5 & 6,6 & 11 & 4,5 & 6,6 & 4,8 & 6,6 \\
\hline 503,3 & 120,6 & 5393m10b & Run2 & 210 & 12,9 & 6,8 & 12 & 9,3 & 4,3 & 9,5 & 4,4 \\
\hline 500,0 & 131,0 & $5404500 b$ & Run1 & 450 & 10,9 & 5,9 & 13 & 5,7 & 3,0 & 5,8 & 3,0 \\
\hline 502,1 & 162,1 & $5435 \mathrm{~m} 10 \mathrm{~b}$ & Run1 & 550 & 10,3 & 6,3 & 14 & 2,8 & 1,3 & 2,7 & 1,3 \\
\hline 751,6 & 56,1 & 7329050 & Run3 & 640 & 5,0 & 16,6 & 15 & 2,6 & 15,3 & 2,8 & 15,3 \\
\hline 752,5 & 77,0 & 7349100 & Run3 & 550 & 8,6 & 11,6 & 16 & 5,1 & 9,1 & 5,6 & 9,1 \\
\hline 748,4 & 95,3 & 7368500 & Run3 & 320 & 13,6 & 12,0 & 17 & 8,6 & 6,1 & 8,8 & 6,3 \\
\hline 753,2 & 121,1 & 7393m10 & Run3 & 230 & 15,7 & 9,1 & 18 & 9,7 & 5,0 & 9,8 & 5,0 \\
\hline 749,6 & 131,9 & 7404500 & Run3 & 270 & 15,8 & 8,7 & 19 & 7,0 & 3,3 & 7,1 & 3,3 \\
\hline 749,2 & 161,8 & $7435 \mathrm{~m} 20$ & Run3 & 480 & 11,9 & 5,4 & 20 & 3,2 & 1,5 & 3,2 & 1,5 \\
\hline
\end{tabular}

Para calcular o erro da dimensão da onda de choque nos vinte testes, experimentais e numéricos, usamos a norma euclidiana, definida por:

$$
\|x\|_{2}:=\left(\sum_{i=1}^{20} x_{i}^{2}\right)^{1 / 2}
$$

onde $x \in R^{20}$.

Para o caso sem refinamento: Seja $r_{1}^{\text {exp }}=\left(r_{1}^{1}, \ldots, r_{1}^{20}\right)$ o vetor comprimento radial experimental para os vinte testes, $r_{1}^{\text {num }}=\left(\mathrm{r}_{1}^{1}, \ldots, \mathrm{r}_{1}^{20}\right)$ o vetor comprimento radial numérico para os vinte testes. De forma análoga obtemos para o vetor comprimento axial. Defina-se o erro relativo radial entre os 20 testes experimentais e numéricos por: 


$$
E_{r_{1}}=\frac{\left\|r_{1}^{\exp }-r_{1}^{\text {num }}\right\|_{2}}{\left\|r_{1}^{\exp }\right\|_{2}} \times 100 \%
$$

e para o erro relativo axial entre os 20 testes experimentais e numéricos por:

$$
E_{r_{2}}=\frac{\left\|r_{2}^{\exp }-r_{2}^{\text {num }}\right\|_{2}}{\left\|r_{2}^{\exp }\right\|_{2}} \times 100 \%
$$

De forma análoga se calcula o erro relativo para o caso com refinamento.

Para o caso sem refinamento de um total de 20 testes, os testes numéricos aproximamse dos testes experimentais (Vieira, 2005) com um erro de $41,10 \%$ para o caso do comprimento radial $r_{1}$ e, 30,47\% para o caso do comprimento axial $r_{2}$. No caso da malha com refinamento o erro diminui muito pouco, conforme se pode ver na Tabela 6.3.

Tabela 6.3 Erro relativo da dimensão da onda de choque entre os resultados numéricos e experimentais (Vieira, 2005).

\begin{tabular}{ccc}
\hline Total de testes: 20 & $E_{r_{1}} \%$ & $E_{r_{2}} \%$ \\
\hline Sem refinamento & 41,10 & 30,47 \\
Com refinamento & 40,30 & 30,24 \\
\hline
\end{tabular}

Outra forma de analisar o comportamento do erro das dimensões da onda de choque é através de gráficos. Para o caso sem refinamento, um total de 20 testes foi esboçado, tanto para o caso experimental, quanto para o caso numérico. Assim na Fig. 6.4a temos o gráfico das dimensões do comprimento radial, $r_{1}$, e na Fig. 6.4b o gráfico para o comprimento axial, $r_{2}$.
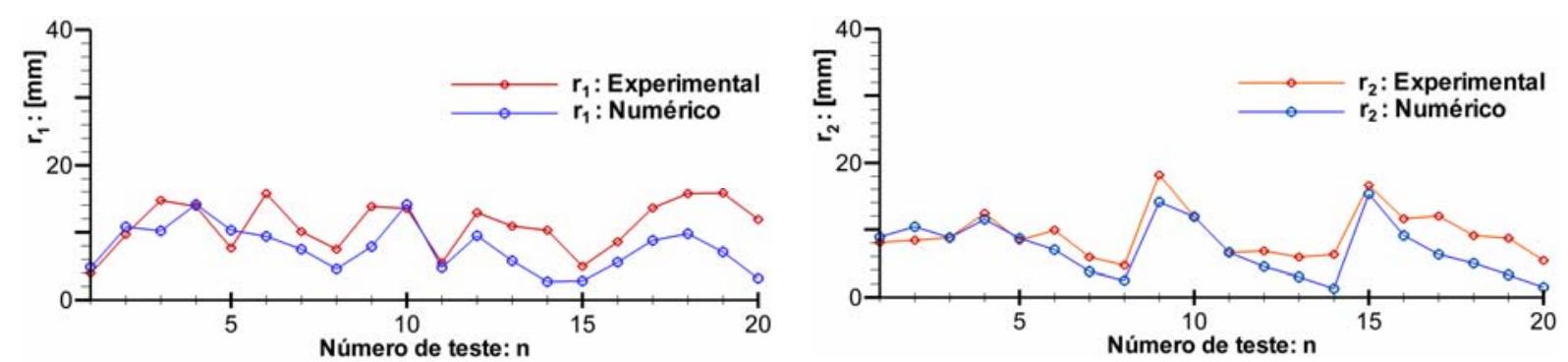

Figura 6.4 Dimensões da onda de choque para o caso sem refinamento num total de 20 testes.

a) Comprimento radial, $r_{1}[\mathrm{~mm}]$. b) Comprimento axial, $r_{2}[\mathrm{~mm}]$. 


\subsubsection{Localização da onda de choque}

Os testes numéricos, com a captura e localização da onda de choque, podem ser vistos para diferentes campos e/ou propriedades termodinâmicas, tais como, por exemplo: - pressão, - temperatura, - título e - número de Mach. Na Fig. 6.5 mostramos de um lado o resultado experimental (VIEIRA, 2005) e do outro lado nossos resultados numéricos; executados todos para o teste número: 5349500 (caso experimental, Vieira (2005)) ou 10 (caso numérico), ou seja:

$$
\left\{P_{0}=497,6[\mathrm{kPa}] ; T_{0}=76,9\left[{ }^{\circ} \mathrm{C}\right] ; P_{\infty}=110[\mathrm{~Pa}]\right\} .
$$
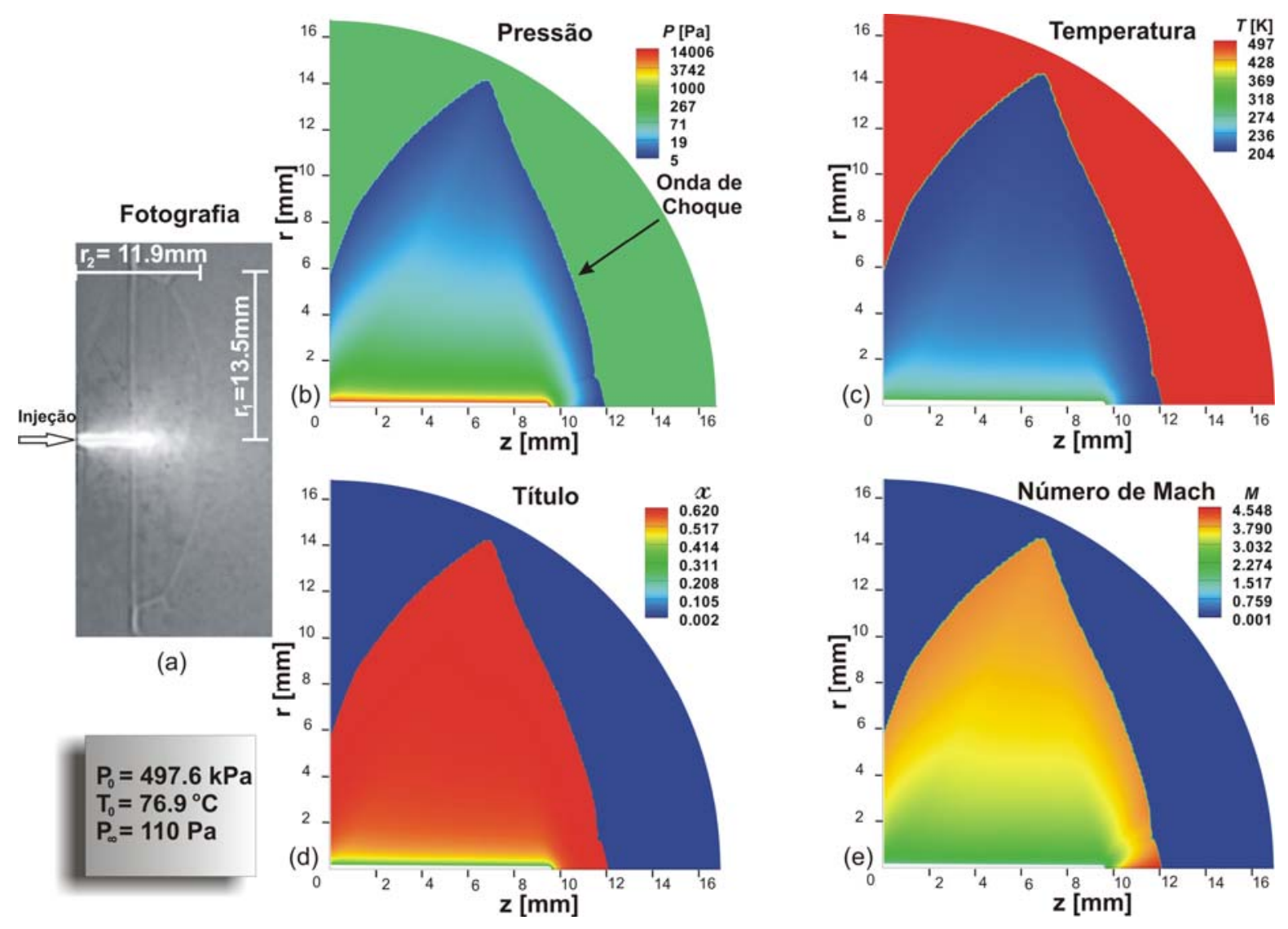

Figura 6.5 Localização da onda de choque em: (a) Fotografia de Vieira (2005); (b) Pressão; (c) Temperatura; (d) Título; (e) Número de Mach.

\subsection{Comparação com Resultados Numéricos}

São comparados os resultados processados pelos códigos ShoWPhasT-2D v1 $e$ ShoWPhasT-2D v2 para as condições de contorno propostas de forma diferente e tempo CPU que utilizam para resolver o problema. 


\subsubsection{Condições de contorno}

As condições de contorno tratadas aqui se referem à Linha de parede e a Linha de simetria. Angelo (2004) estuda estas condições de contorno em duas etapas: primeiro emprega, o princípio de impermeabilidade para o componente da velocidade perpendicular à direção da parede, isto é, se anula. A outra componente seguirá a direção paralela à parede, por ser o fluido invíscido. Na segunda etapa, implementa uma técnica que consiste em realizar uma expansão (ou compressão) isoentrópica de Prandtl-Meyer através da rotação correspondente ao ângulo do vetor velocidade.

Neste trabalho, as condições de contorno usadas seguem somente o principio de impermeabilidade, não usamos o segundo passo porque algumas fórmulas foram deduzidas usando a equação de estado para gases perfeitos. As condições de contorno são apresentadas na Seção (3.3).

O estudo especial do vetor velocidade no nó inicial da malha (vértice $A$ na malha física ou vértice $A^{\prime}$ na malha computacional, veja Fig. 3.2) foi um dos procedimentos que fizeram nossos resultados diferentes. Esta mudança fez aparecer uma linha de corrente diferente da linha de Angelo (2004) e mais próximo aos resultados de Vieira (2005) que, sob o efeito da parede, faz aparecer uma onda reflexiva, dando assim inicio à onda de choque. Nas figuras 6.6a, 6.6b e 6.6c apresentamos esses resultados, observe a semelhança entre a Fig. 6.6b e a Fig. 6.6c no correspondente à onda de choque formada na parede. A Fig. 6.6c está completa sem a condição de simetria e representa o ensaio 5329500, Vieira (2005).
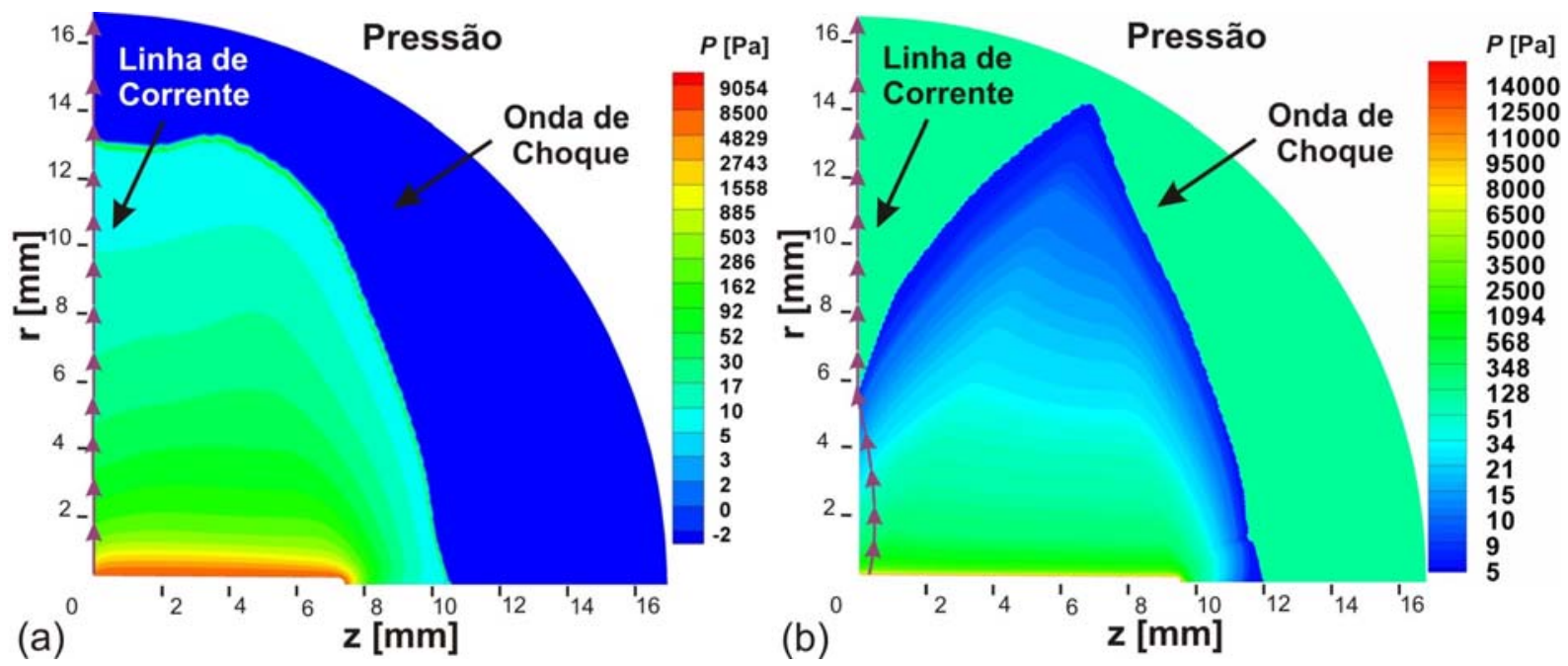


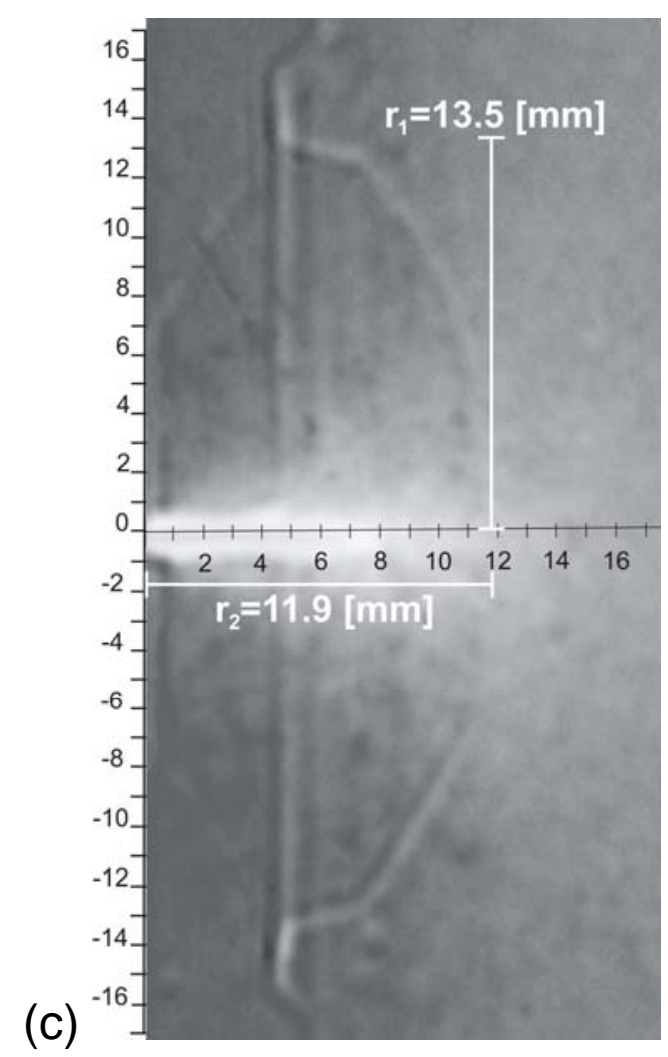

Figura 6.6 Linha de corrente e onda de choque para o Teste 10 (Serie RUN3, ensaio 5329500, $P_{0}=497,6[\mathrm{kPa}] ; T_{0}=76,9\left[{ }^{\circ} \mathrm{C}\right] ; P_{\infty}=110[\mathrm{~Pa}]$ ). (a) Resultado com o código ShoWPhasT-2D $v 1$, Angelo (2004). (b) Resultado deste trabalho com o código ShoWPhasT-2D v2. (c) Resultado experimental, Vieira (2005).

\subsubsection{Tempo computacional}

Cada um dos testes foi realizado em um computador: AMD Atlon(tm) 64X2 Dual Core Processor 4200+ 2.46 GHz. O tempo CPU, foi comparado com o tempo que demora o software ShoWPhasT-2D v2 e o tempo que demora o software ShoWPhasT-2D v1. A versão 2 de nosso software divide o problema da expansão bifásica de jatos altamente superaquecidos em três grupos:

1. A malha computacional.

2. A expansão da mistura bifásica.

3. A captura da onda de choque.

Na Tabela 6.4, mostramos esses resultados para o Teste 10. Por exemplo, o tempo total que demora o código ShoWPhasT-2D v1 (Angelo, 2004), em resolver este teste é: 2 horas, 16 minutos, 9 segundos e 656 milisegundos, em quanto que, nosso código ShoWPhasT-2D v2, resolve para o caso sem refinamento, em: 17 segundos, 417 milisegundos, e para o caso com 
refinamento demora: 28 segundos e 23 milisegundos. Concluindo que existe uma grande diferença de tempo CPU entre os dois códigos, diferença que se deve à substituição no código (Angelo, 2004) da equação de estado de Lee-Kesler (equação algébrica não linear) pela tabela das propriedades termodinâmicas do iso-octano (estudado no Apêndice D).

Tabela 6.4 Tempo CPU de execução do código.

\begin{tabular}{crcc}
\hline & \multirow{2}{*}{ ShoWPhasT-2D v1 } & \multicolumn{2}{c}{ ShoWPhasT-2D v2 } \\
\cline { 3 - 4 } & & Sem Refinamento & Com Refinamento \\
\hline Malha & $2: 08: 44: 875$ & $14: 656$ & $23: 750$ \\
Expansão bifásica & & $01: 921$ & $03: 218$ \\
Captura & $07: 24: 781$ & $00: 640$ & $01: 125$ \\
Tempo total & $2 h: 16 m: 9 s: 656 m s$ & $17 s: 417 m s$ & $28 s: 023 m s$ \\
\hline
\end{tabular}

Portanto, neste capítulo se conseguiu obter resultados numéricos usando o código computacional ShoWPhasT-2D v2, para o fenômeno de evaporação rápida de jatos de líquidos metaestáveis em regime permanente. Estes resultados numéricos foram comparados com resultados experimentais de Vieira (2005). 


\section{RESULTADOS NUMÉRICOS: CASO FALSO TRANSITÓRIO}

Os fenômenos estacionários podem ser resolvidos numericamente por métodos estacionários ou não estacionários. Quando estes fenômenos estacionários são resolvidos por métodos não estacionários, estes são chamados de método ou modelo de falso transitório. Esta técnica consiste em acrescentar um termo temporal às equações que governam o fenômeno estacionário e resolvê-las como se fossem não estacionárias até atingir o regime permanente. É evidente que estas soluções temporais, até antes de atingir o regime permanente, não tem significado físico e sim numérico.

O código computacional "Dispersion-Controlled Dissipative - Two-Dimensional version 1", $D C D-2 D v 1$, usa o esquema DCD de segunda ordem, o qual é um modelo não estacionário de captura de choque (“shock-capturing”). Este código foi implementado para resolver fenômenos não estacionários 2D, modelados matematicamente por sistemas leis de conservação hiperbólicas. Um algoritmo em pseudocódigo do $D C D-2 D$ v1 foi elaborado no Apêndice (A.3).

O fenômeno de evaporação rápida (“flashing”) de jatos de líquidos superaquecidos na expansão bifásica é um problema estacionário (mais detalhes no Capítulo 4), o qual será resolvido pelo código $D C D-2 D$ v1 com as restrições para um modelo de falso transitório.

Neste capítulo analisaremos a validação do código $D C D-2 D v 1$, segundo o modelo de falso transitório, para diferentes geometrias 2D, nos seguintes problemas: reflexão de uma onda de choque, bocal convergente-divergente, bocal supersônico, bocal supersônico com vetor velocidade oblíquo, todos eles testados para o ar. Como também, resolveremos o fenômeno da expansão bifásica para os problemas: Teste11 e Teste 04, sendo o fluido teste o iso-octano com tabelas geradas a partir da equação de estado de Lee-Kesler (uso de tabela das propriedades termodinâmicas na saturação). São mostrados gráficos das simulações numéricas para as diferentes propriedades termodinâmicas, e por último apresentamos comparações dos resultados numéricos e experimentais da geometria da onda de choque.

\subsection{Validação do Código Computacional DCD-2D v1}

Com a finalidade de validar o código computacional $D C D-2 D$ v1, segundo o modelo de falso transitório, foram realizados testes numéricos para diferentes fenômenos 2D e quase- 
unidimensional. O fluido teste usado foi o ar (com equação de estado dos gases perfeitos, conforme mostrado no Cap. (3.5.1)) com velocidades subsônicas e supersônicas.

Referente às malhas usadas nos diferentes testes, algumas são geométricas (obtidas por cálculos algébricos) e outras geradas por transformações de coordenadas biunívocas, assim como estudado no Cap. (3.4). O subscrito usado para denotar uma malha de dimensão $[(N+2) \times(M+2)]$ nós, é: $(i=\overline{0, N+1} ; j=\overline{0, M+1})$, onde $i$ denota os pontos das linhas paralelas à Linha inicial, $j$ os pontos das linhas que seguem aproximadamente a direção do escoamento e $M, N$ números inteiros positivos fixos. Por exemplo, quando queremos fixar uma linha é só fixar um subscrito: $i$ ou $j$; e quando queremos fixar um nó fixamos ambos subscritos: $i$ e $j$.

As condições iniciais usadas no interior do domínio podem ser arbitrárias, desde que não fujam muito da realidade ou causem situações impossíveis fisicamente. O código $D C D$ 2D v1 suporta todas estas condições iniciais, porém, para problemas de "shock-capturing" empregam-se condições inicias que incluam ondas de choques (normais ou oblíquas) posicionadas próximas do inicio do escoamento, assim ocorre um ganho de tempo computacional e "garantimos” convergência da solução numérica.

A expressão “Uso EE_GP”, significa que se usou a equação de estado dos gases perfeitos para calcular uma variável termodinâmica. Por outro lado, a equação da velocidade do som, $c$, é dada por:

$$
c=\sqrt{\gamma \frac{p}{\rho}}
$$

onde a razão entre calores específicos, $\gamma$ é igual a 1.4.

Cada um dos testes foi executado num computador: AMD Atlon(tm) 64X2 Dual Core Processor $5200+2.73 \mathrm{GHz}$.

\subsubsection{Reflexão de uma onda de choque}

\section{- Descrição do problema}

Considere-se o ar supersônico escoando na direção horizontal de um canto de $16^{\circ}$ de inclinação (ângulo de deflexão $\theta$ ) e paredes sólidas $\overline{B C}$ e $\overline{A D}$ (comprimento 14 [m]), assim 
como mostra a Fig. 7.1. O escoamento atravessa a entrada $\overline{A B}$ (comprimento 7 [m]) e a saída $\overline{D C}$ dividindo o canto em três regiões I, II e III. A atravessar o escoamento se gera uma onda de choque oblíqua incidente sobre a parede $\overline{A D}$. Para satisfazer a condição de que as linhas de corrente devem ser paralelas às superfícies sólidas, a onda de choque deve ser refletida num ângulo tal que o paralelismo seja satisfeito.

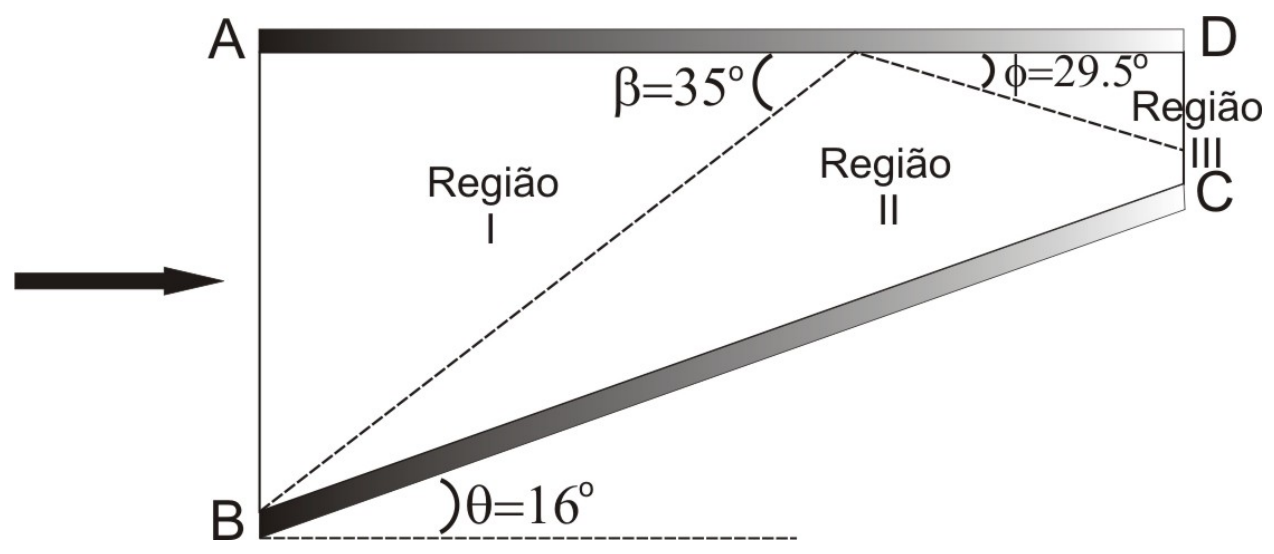

Figura 7.1 Reflexão de uma onda de choque.

\section{- Solução analítica}

Estes dados são fornecidos por Anderson (1990, p. 120) e reproduzidos na segunda coluna da Tabela 7.1.

\section{- Condições iniciais}

As condições iniciais correspondem ao tempo $t=0$ na discretização temporal. Então devemos conhecer os valores das propriedades termodinâmicas nas variáveis espaciais do domínio, o qual é dividido em nós do interior e nós do contorno.

\section{- Condições de contorno}

O contorno do canto esta formado pelas seguintes linhas: linha inicial ( $\overline{A B}$ ), linha de parede inferior $(\overline{B C})$, linha de parede superior $(\overline{A D})$ e linha final $(\overline{C D})$. As condições de contorno físicas e numéricas para as variáveis primitivas são dadas na Tabela 7.1. Todas as condições de contorno numéricas são extrapolações de ordem zero. 
Depois de executar o código $D C D-2 D$ v1 obtivemos a solução numérica mostrada na Tabela 7.2 para a linha $(i=25 ; j=\overline{0.388})$ da malha.

Tabela 7.1 Condições de contorno para o problema de reflexão da onda de choque.

\begin{tabular}{l|c|c|c|c}
\hline \multirow{2}{*}{$\begin{array}{c}\text { Variáveis } \\
\text { primitivas }\end{array}$} & \multicolumn{4}{|c}{ Condições de contorno físicas e numéricas } \\
\cline { 2 - 5 }$M$ & Linha inicial & Parede inferior & Parede superior & Linha final \\
\hline$T[\mathrm{~K}]$ & 2,8 & $V / C$ & $V / C$ & $V / C$ \\
$p[\mathrm{~Pa}]$ & 288,33 & Uso EE_GP & Uso EE_GP & Uso EE_GP \\
$\mathrm{u}[\mathrm{m} / \mathrm{s}]$ & 101,325 & Uso EE_GP & Uso EE_GP & $p_{i, M+1}=p_{i, M}$ \\
$\mathrm{v}[\mathrm{m} / \mathrm{s}]$ & $M \times C$ & $\mathrm{u}_{N+1, j}=\mathrm{u}_{N, j}$ & $\mathrm{u}_{0, j}=\mathrm{u}_{1, j}$ & $\mathrm{u}_{i, M+1}=\mathrm{u}_{i, M}$ \\
$\rho\left[\mathrm{kg} / \mathrm{m}^{3}\right]$ & Uso EE_GP & $\rho_{N+1, j}=\rho_{N, j}$ & $\rho_{0, j}=\rho_{1, j}$ & $\rho_{i, M+1}=\rho_{i, M}$ \\
$e[\mathrm{~J} / \mathrm{kg}]$ & Uso EE_GP & $e_{N+1, j}=e_{N, j}$ & $e_{0, j}=e_{1, j}$ & Uso EE_GP \\
\hline
\end{tabular}

Tabela 7.2 Solução analítica e numérica da reflexão da onda de choque.

\begin{tabular}{|c|c|c|c|c|c|}
\hline \multirow{2}{*}{$\begin{array}{c}\text { Ângulo de deflexão: } \\
\qquad \theta=16^{\circ}\end{array}$} & \multicolumn{2}{|c|}{ Solução analítica } & \multicolumn{3}{|c|}{ Solução numérica } \\
\hline & Região: II & Região: III & & Região: II & Região: III \\
\hline Mach & 2.053 & 1.45 & & 2.05 & 1.44 \\
\hline Temperatura [K] & 400.21 & 517.78 & & 400.23 & 523.7 \\
\hline Pressão [Pa] & 285.74 & 662.67 & & 287.2 & 692.3 \\
\hline Onda incidente: $\beta$ & $35^{\circ}$ & & $34.9^{\circ}$ & & \\
\hline Onda de reflexão: $\phi$ & $29.5^{\circ}$ & & $29^{\circ}$ & & \\
\hline Número de nós da malha & & & $\begin{array}{c}100 \times 390= \\
39000\end{array}$ & & \\
\hline Número de iterações & & & 6500 & & \\
\hline Tempo computacional & & & 01:46:36:484 & & \\
\hline
\end{tabular}

\section{- Distribuição das propriedades termodinâmicas}

Na Fig. 7.2a temos o domínio físico do problema com malha obtida geometricamente, esta malha é muito mais fina da que se usou para resolver o problema. A malha usada no código é de 3900 nós $(100 \times 390=39000)$. As figuras $7.2 b, 7.2$ c e $7.2 d$ representam a distribuição de pressões, temperaturas e número de Mach, respectivamente. Cada uma delas 
contém a onda de choque oblíqua e sua reflexão, observe também que o ponto de reflexão sobre a parede sólida superior se dá aos 10.617 [m].

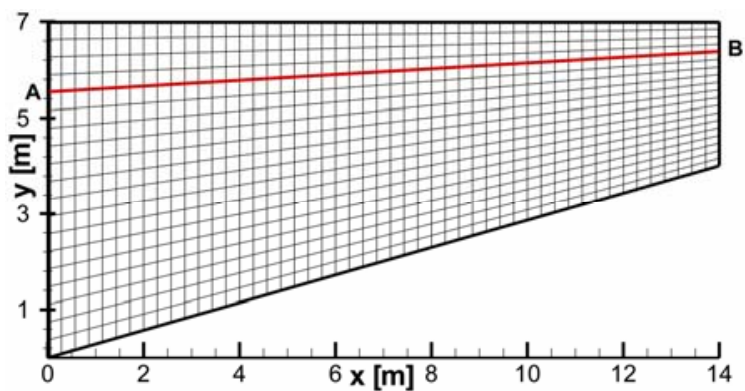

(a)

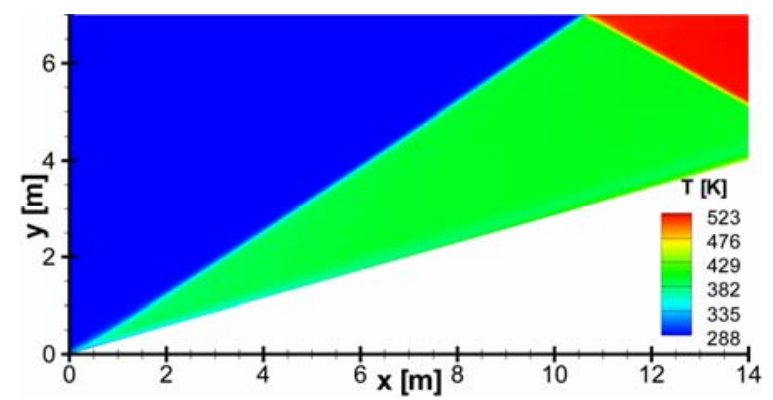

(c)

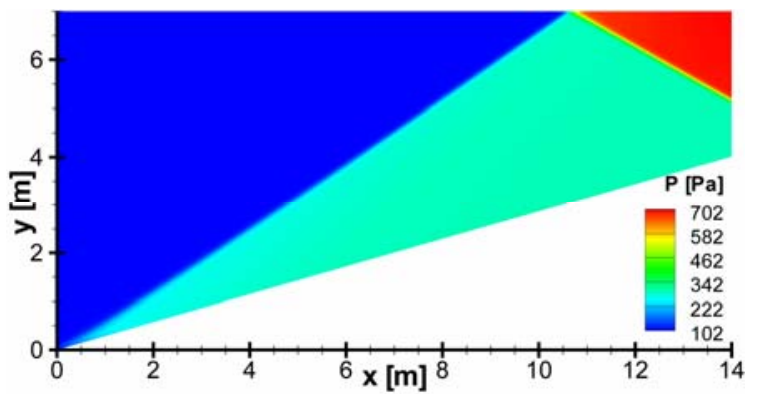

(b)

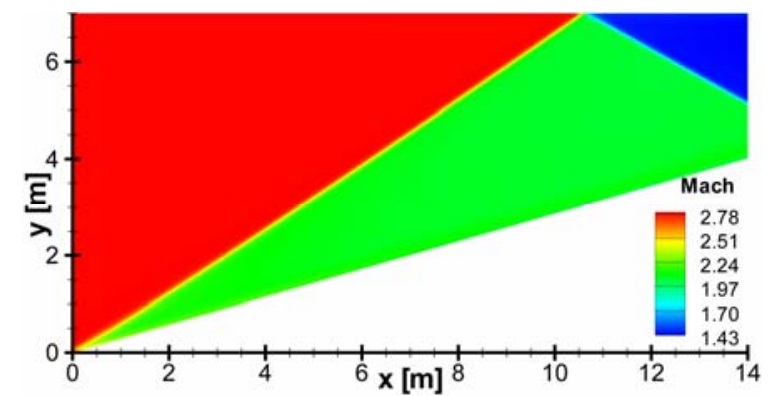

(d)

Figura 7.2 Reflexão de uma onda de choque. (a) Geometria com malha e a linha fixa $\overline{A B}$. (b) Distribuição de pressões. (c) Distribuição de temperaturas. (d) Número Mach.

\section{- $\quad$ Perfis das propriedades termodinâmicas}

Na Fig. 7.3a mostramos o gráfico da pressão versus número de iterações de um ponto arbitrário do domínio físico, por exemplo, neste teste o ponto em questão é $(i=25 ; j=349)$. O número de iterações está diretamente relacionado com o tempo computacional que o código demora em resolver o problema. Daí pode-se dizer que temos os valores da pressão num ponto variando ao longo do tempo. Assim, neste problema podemos observar que o regime permanente foi atingido após 6500 iterações. Para mostrar os perfis das propriedades termodinâmicas usamos uma linha da malha do domínio físico a qual, é fixa e segue a direção do escoamento, ou seja, para um $i=i_{0}$ fixo e $j=\overline{0, M}$ variável. Essa linha é arbitrária e inicia na entrada do domínio e finaliza na saída. Neste problema a linha da malha escolhida é o segmento de reta $\overline{A B}$ que mostramos na Fig. 7.2a e que corresponde aos pontos 
$(i=25 ; j=\overline{0,388})$. As seguintes figuras $7.3 \mathrm{~b}, 7.3 \mathrm{c}$ e $7.3 \mathrm{~d}$ representam o perfil da pressão, temperatura e número de Mach, respectivamente, após ter atingido o estado permanente.

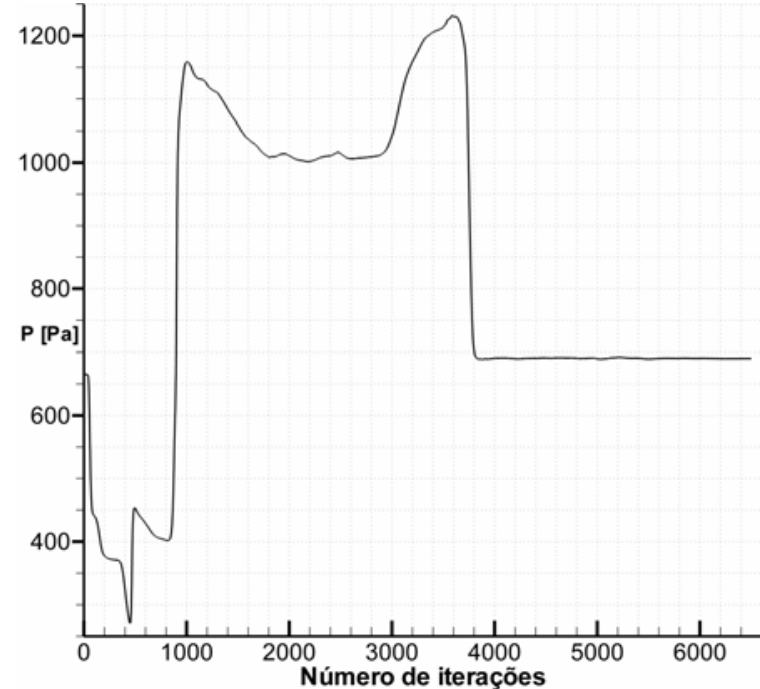

(a)

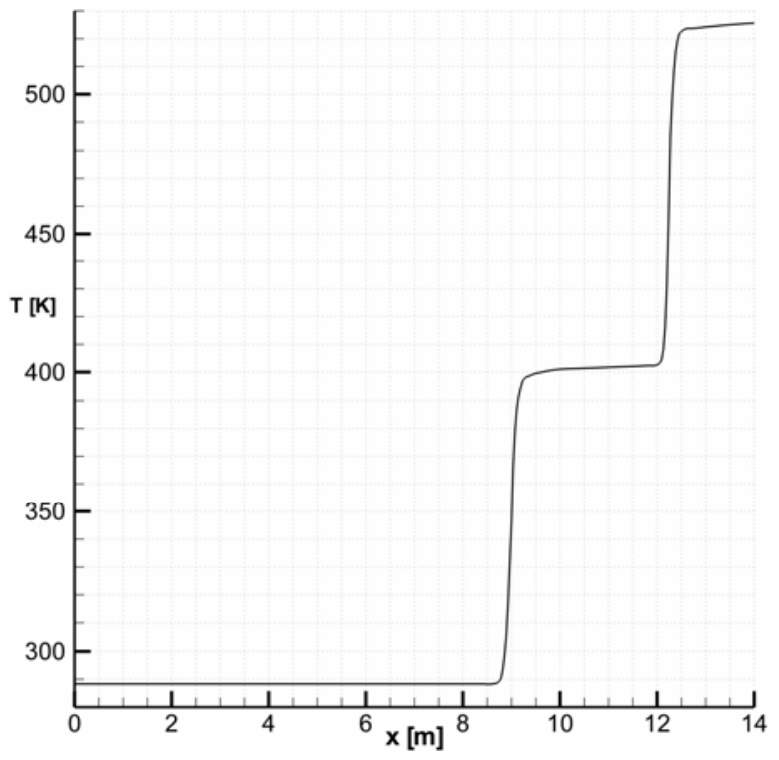

(c)

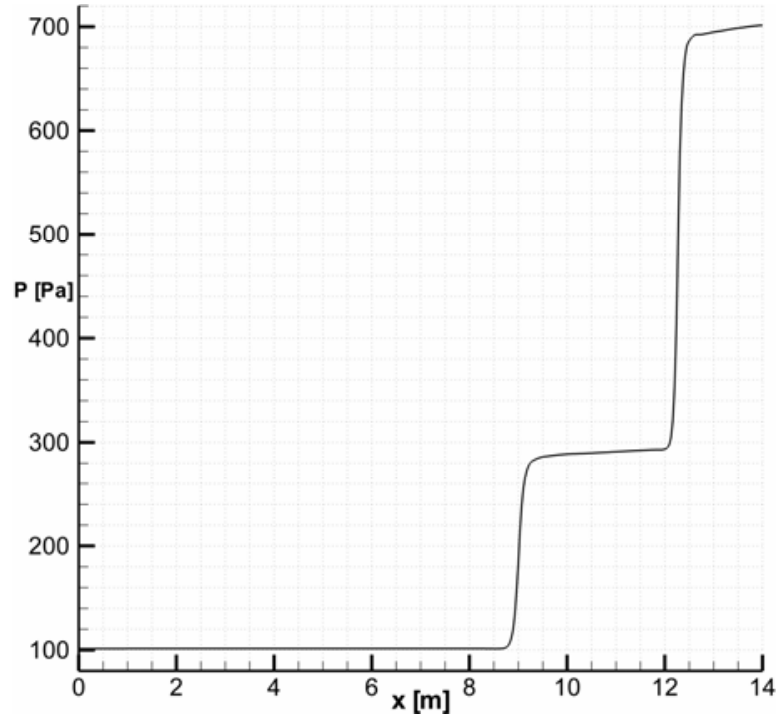

(b)

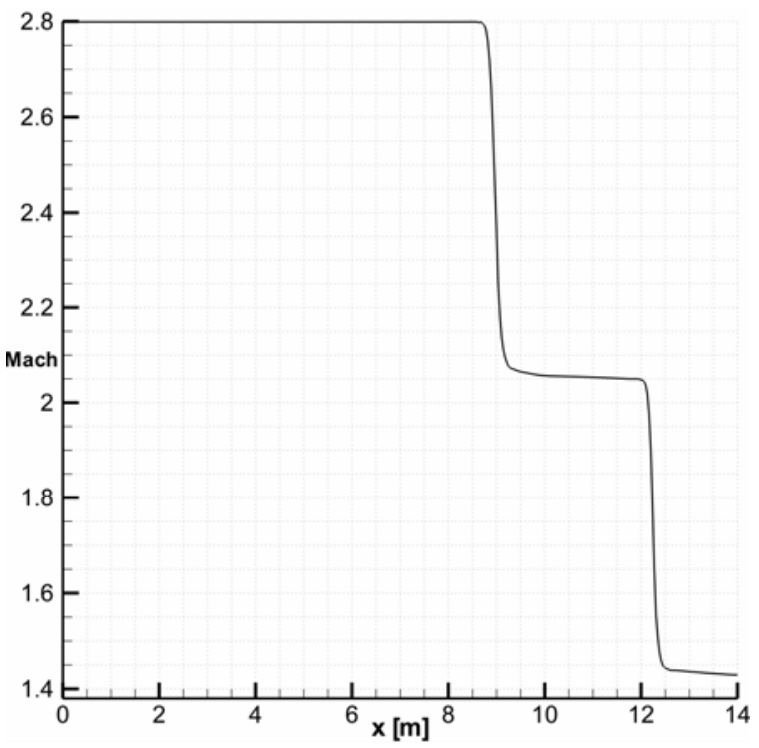

(d)

Figura 7.3 (a) Comportamento da pressão versus número de iterações. Os seguintes gráficos: (b) Pressão, (c) Temperatura e (d) Número Mach atingiram o regime permanente. 


\subsubsection{Bocal convergente-divergente}

\section{- Descrição do problema}

O problema do bocal convergente-divergente é quase unidimensional sendo a razão de área $A_{s} / A^{*}=5.95$, onde $A^{*}$ é área na garganta e $A_{s}$ é área na saída. Este problema é idêntico ao exemplo de Anderson (1995, p. 356). As condições de reservatório e dados de entrada são mostradas na Tabela 7.4. A região do bocal convergente-divergente é dada por:

$$
D=\left\{(x, y) \in R^{2}: 0 \leq x \leq 3 \wedge|y| \leq(1 / 8)\left[1+2.2(x-1.5)^{2}\right]\right\}
$$

Resultados numéricos são mostrados na Tabela 7.4. Observe que unicamente a pressão apresenta um erro relativamente alto.

\section{- $\quad$ Condições de contorno}

Estão especificadas na Tabela 7.3.

Tabela 7.3 Condições de contorno para o bocal convergente-divergente.

\begin{tabular}{l|c|c|c|c}
\hline \multirow{2}{*}{$\begin{array}{c}\text { Variáveis } \\
\text { primitivas }\end{array}$} & \multicolumn{4}{|c}{ Condições de contorno físicas e numéricas } \\
\cline { 2 - 5 }$M$ & Linha inicial & Parede inferior & Parede superior & Linha final \\
\hline$T[\mathrm{~K}]$ & 0,0982 & $V / C$ & $V / C$ & $V / C$ \\
$p[\mathrm{~Pa}]$ & 320,0 & Uso EE_GP & Uso EE_GP & Uso EE_GP \\
$\mathrm{u}[\mathrm{m} / \mathrm{s}]$ & $M 00000,0$ & Uso EE_GP & Uso EE_GP & $p_{i, M+1}=p_{i, M}$ \\
$\mathrm{v}[\mathrm{m} / \mathrm{s}]$ & $M \times C$ & $\mathrm{u}_{N+1, j}=\mathrm{u}_{N, j}$ & $\mathrm{u}_{0, j}=\mathrm{u}_{1, j}$ & $\mathrm{u}_{i, M+1}=\mathrm{u}_{i, M}$ \\
$\rho\left[\mathrm{kg} / \mathrm{m}^{3}\right]$ & Uso EE_GP & $\rho_{N+1, j}=\rho_{N, j}$ & $\rho_{0, j}=\rho_{1, j}$ & $\rho_{i, M+1}=\rho_{i, M}$ \\
$e[\mathrm{~J} / \mathrm{kg}]$ & Uso EE_GP & $e_{N+1, j}=e_{N, j}$ & $e_{0, j}=e_{1, j}$ & Uso EE_GP \\
\hline
\end{tabular}


- Solução numérica

Tabela 7.4 Solução do bocal convergente-divergente.

\begin{tabular}{|c|c|c|c|c|c|}
\hline Condições de Reservatório: & \multirow[t]{2}{*}{ Entrada } & Solução analítica & \multicolumn{2}{|c|}{ Solução numérica } & \multirow[t]{2}{*}{ Erro \% } \\
\hline$P_{0}=600[\mathrm{kPa}], \quad T_{0}=320[\mathrm{~K}]$ & & Saída & & Saída & \\
\hline Mach & 0.098 & 0.143 & & 0.140 & 2.10 \\
\hline Temperatura: $T / T_{0}$ & 1.000 & 0.996 & & 0.985 & 1.10 \\
\hline Pressão: $P / P_{0}$ & 1.000 & 0.678 & & 0.791 & 16.67 \\
\hline Posição da onda de choque [m] & & & 2.15 & & \\
\hline Número de nós da malha & & & $39 \times 181=7059$ & & \\
\hline Tempo computacional & & & $00: 44: 43: 312$ & & \\
\hline
\end{tabular}

A malha na Fig. 7.4a é menos refinada que a malha que se usou na solução do problema, a malha que se usou é de $(39 \times 181=7059)$ nós. As seguintes figuras $7.4 \mathrm{~b}, 7.4 \mathrm{c}$ e 7.4d representam a distribuição de pressão, temperatura e número Mach, respectivamente. Em cada uma delas se observa a formação de uma onda de choque normal posicionada no ponto $2.15[\mathrm{~m}]$.

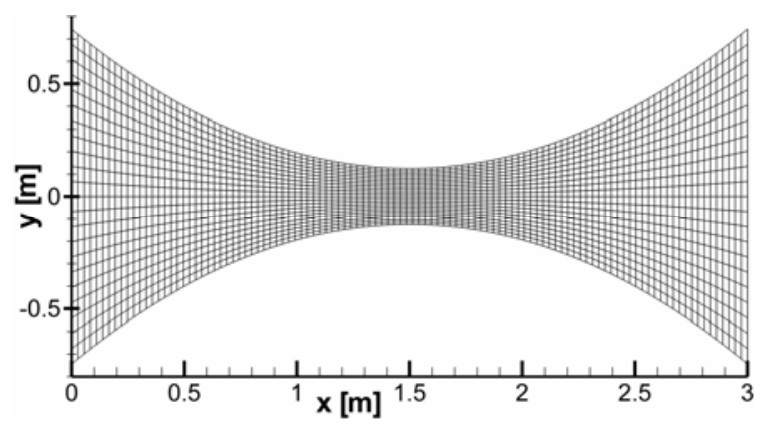

(a)

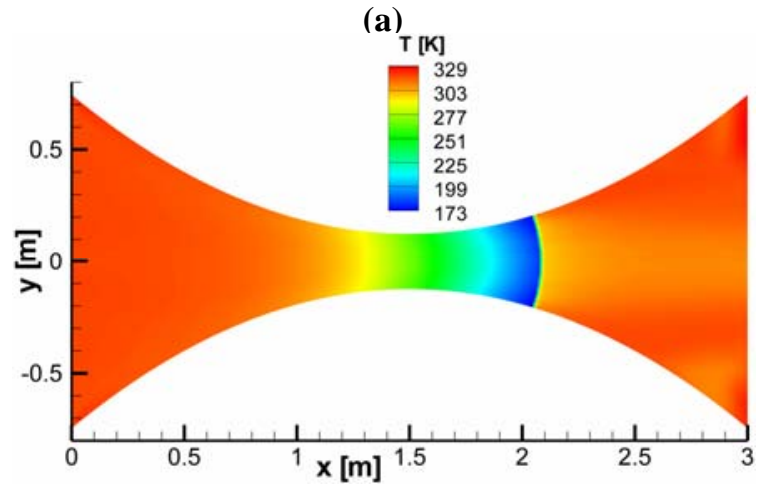

(c)

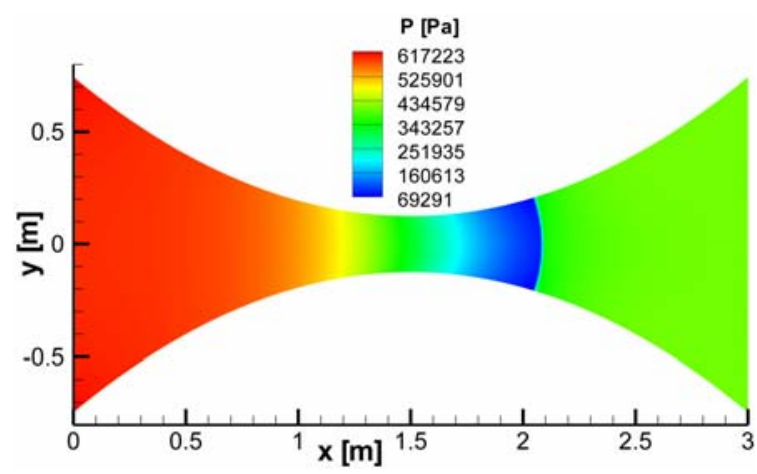

(b)

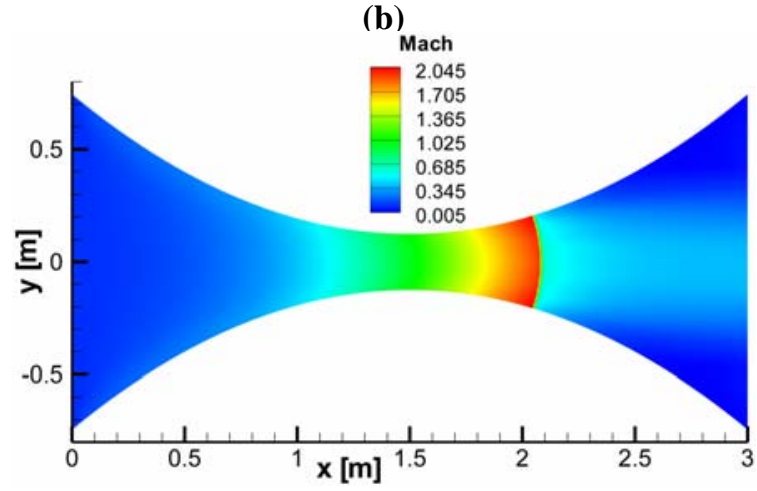

(d)

Figura 7.4 Bocal convergente-divergente. (a) Geometria com malha. (b) Distribuição de pressões. (c) Distribuição de temperaturas. (d) Número Mach. 
A Fig. 7.5a representa o gráfico pressão versus número de iterações. Observe que o problema atinge o estado permanente em 15000 iterações. O ponto em questão é: $(19,161)$. O tempo computacional é de 44 minutos, 43 segundos mais 312 milisegundos.

A linha da malha usada para os perfis é $(i=19 ; j=\overline{0,181})$. As próximas figuras $7.5 a$, 7.5b e 7.5c representam os perfis da pressão, temperatura e número Mach, respectivamente. Observe que os perfis são as relações $P / P_{0}, T / T_{0}$, onde $P_{0}, T_{0}$ são a pressão e temperatura de reservatório.

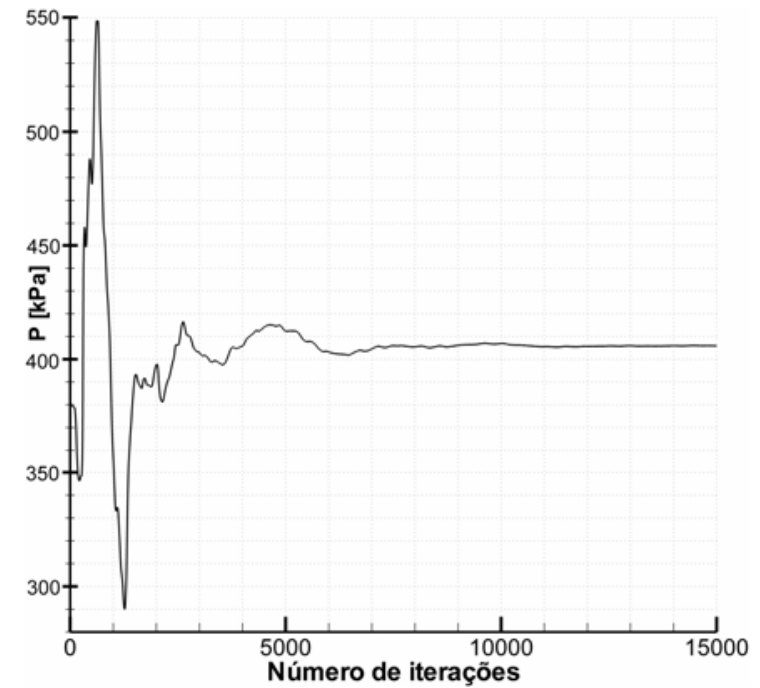

(a)

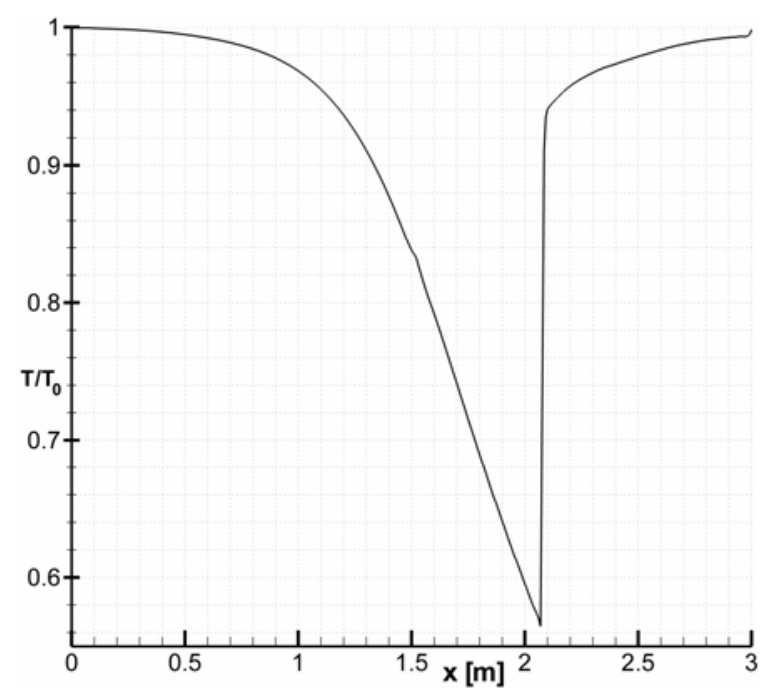

(c)

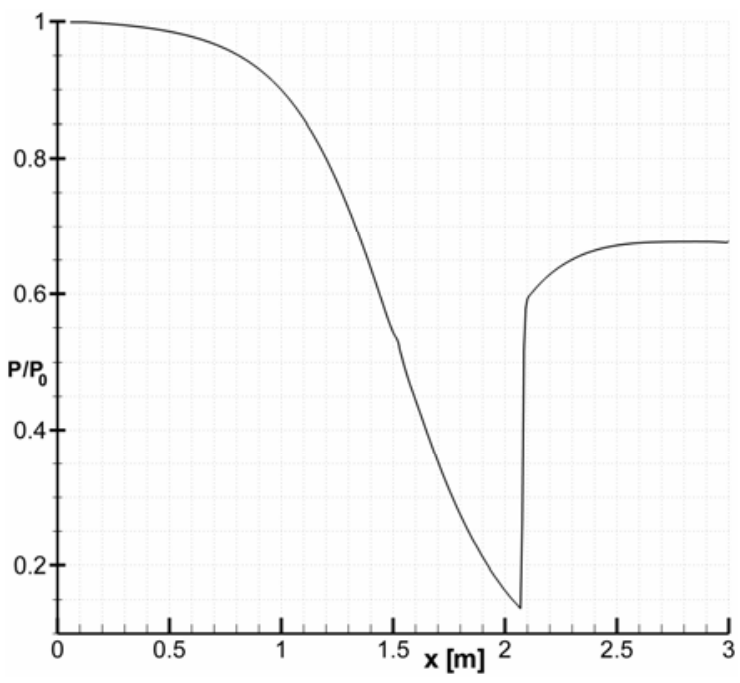

(b)

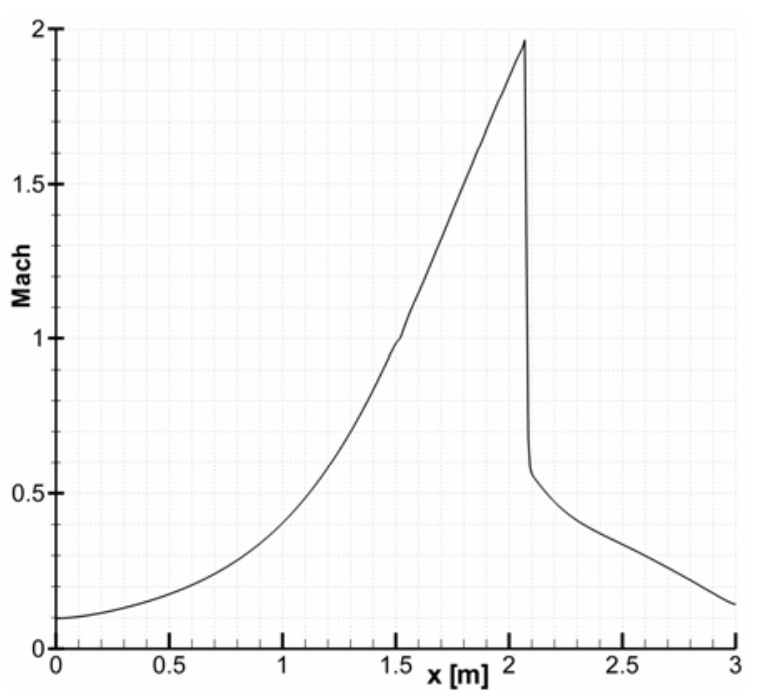

(d)

Figura 7.5 (a) Comportamento da pressão versus número de iterações para um nó fixo da malha. Os seguintes gráficos: (b) Pressão, (c) Temperatura e (d) Número Mach atingiram o regime permanente e indicam sua distribuição espacial ao longo de uma linha fixa da malha. 


\subsubsection{Bocal supersônico}

O problema do bocal supersônico (divergente) foi estudado por ser geometricamente semelhante à geometria da expansão bifásica, além disso, porque poderíamos colocar o valor de Mach 1.0 na entrada. Os dados deste problema, inclusive a relação de áreas, foram tomados de Anderson (1995, p. 356).

Para resolvê-lo numericamente usamos as mesmas condições de reservatório do problema do bocal convergente-divergente. Os dados de entrada são mostrados na Tabela 7.5 abaixo. A relação de áreas é: $A_{s} / A_{e}=5.95 / 1.0=5.95$, onde $A_{e}$ é área na entrada e $A_{s}$ é área na saída. A região do bocal supersônico é dada por:

$$
D=\left\{(x, y) \in R^{2}: 1.5 \leq x \leq 3 \wedge|y| \leq(1 / 8)\left[1+2.2(x-1.5)^{2}\right]\right\}
$$

e na saída fixamos o valor do número de Mach, a invés da pressão. Os resultados obtidos foram bastante favoráveis, o qual se mostra na Tabela 7.5.

Tabela 7.5 Solução do bocal supersônico.

\begin{tabular}{|c|c|c|c|c|c|c|}
\hline Condições de Reservatório: & \multirow[t]{2}{*}{ Entrada } & \multicolumn{2}{|c|}{ Solução analítica } & \multicolumn{2}{|c|}{ Solução numérica } & \multirow[t]{2}{*}{ Erro \% } \\
\hline$P_{0}=600[\mathrm{kPa}], \quad T_{0}=320[\mathrm{~K}]$ & & & Saída & & Saída & \\
\hline Mach & 1.0 & & 0.143 & & 0.143 & \\
\hline Temperatura: $T / T_{0}$ & 0.8333 & & 0.996 & & 0.985 & 1.10 \\
\hline Pressão: $P / P_{0}$ & 0.5283 & & 0.986 & & 0.925 & 6.19 \\
\hline Posição da onda de choque [m] & & 2.1 & & 2.17 & & 2.38 \\
\hline Número de nós da malha & & & & $39 \times 181=7059$ & & \\
\hline Tempo computacional & & & & $00: 44: 43: 312$ & & \\
\hline
\end{tabular}

A malha gerada no domínio do bocal supersônico foi obtida geometricamente e na Fig. 7.6a mostrada de forma simplificada, já que a malha que usamos foi de $(91 x 91=8281)$ nós. Nas seguintes figuras 7.6b, 7.6c e 7.6d mostram-se a distribuição de pressões, temperatura e número Mach, respectivamente. Observe que a onda de choque normal é mais reta que a onda de choque no bocal convergente-divergente, isto se deve à relação de área que é maior em relação ao outro problema do bocal convergente-divergente. 


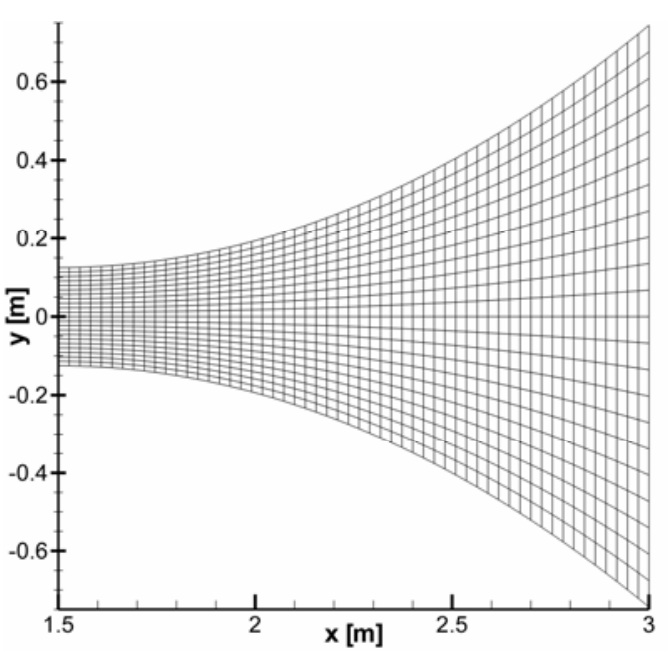

(a)

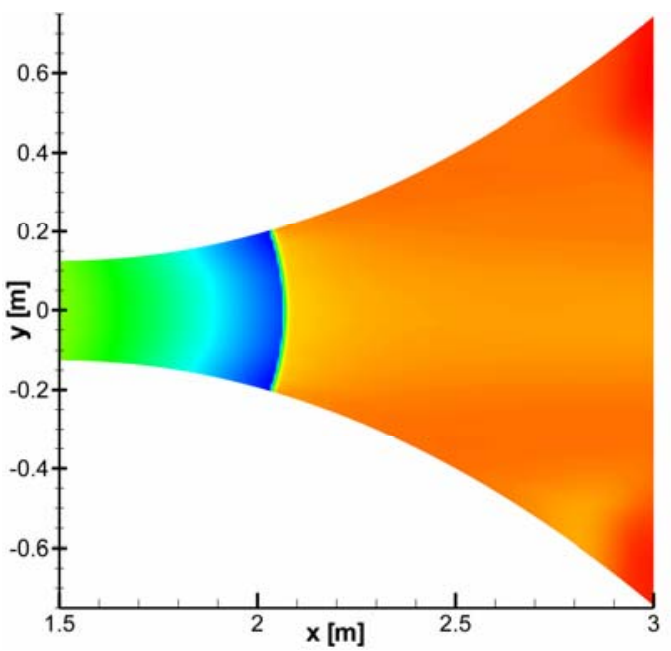

(c)

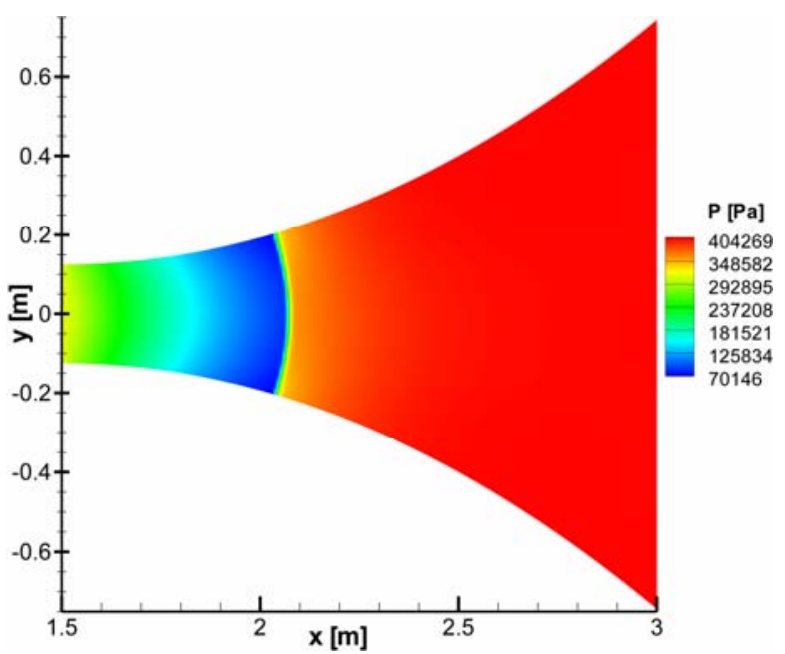

(b)

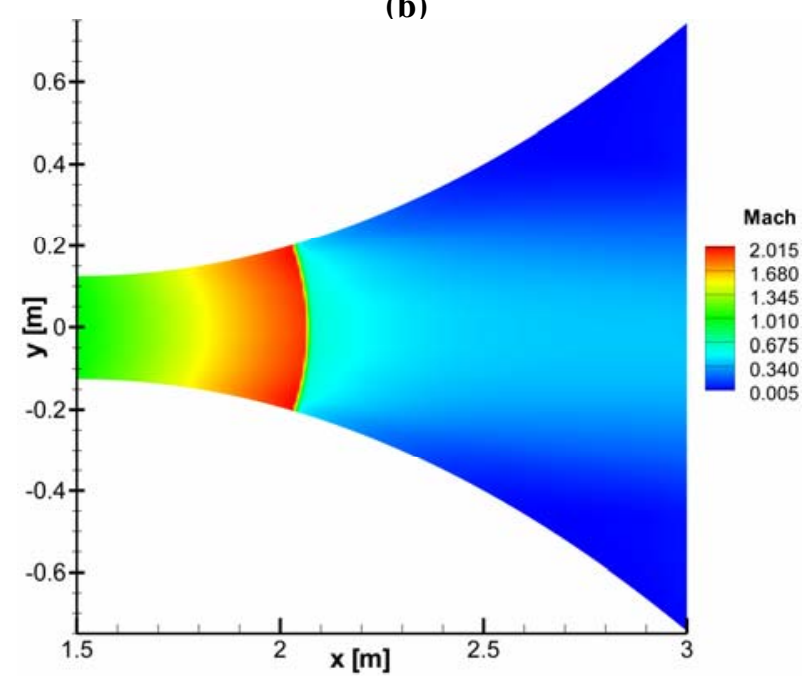

(d)

Figura 7.6 Bocal supersônico. (a) Geometria com malha. (b) Distribuição de pressões. (c) Distribuição de temperaturas. (d) Número Mach.

Na Fig. 7.7a observa-se que o estado de regime permanente foi atingido após 10000 iterações, conforme mostrado para nó da malha localizado em $(45,80)$. Nas figuras seguintes 7.7b, 7.7c e 7.7d, são mostrados os perfis da razão de pressões, temperaturas e número Mach, respectivamente. Estes perfis seguem a linha $(i=45 ; j=\overline{0,91})$ da malha, onde $j$ segue aproximadamente a direção do escoamento. 


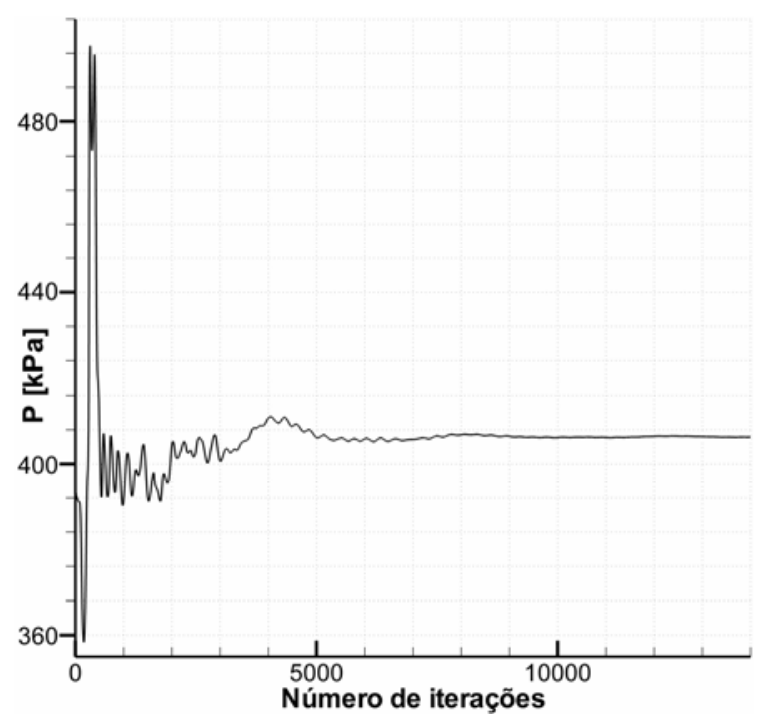

(a)

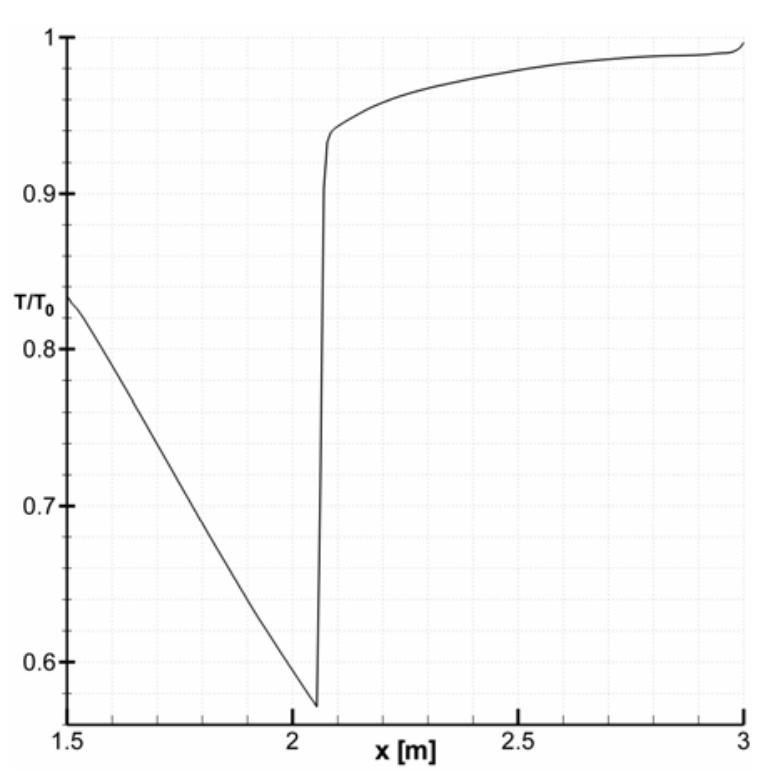

(c)

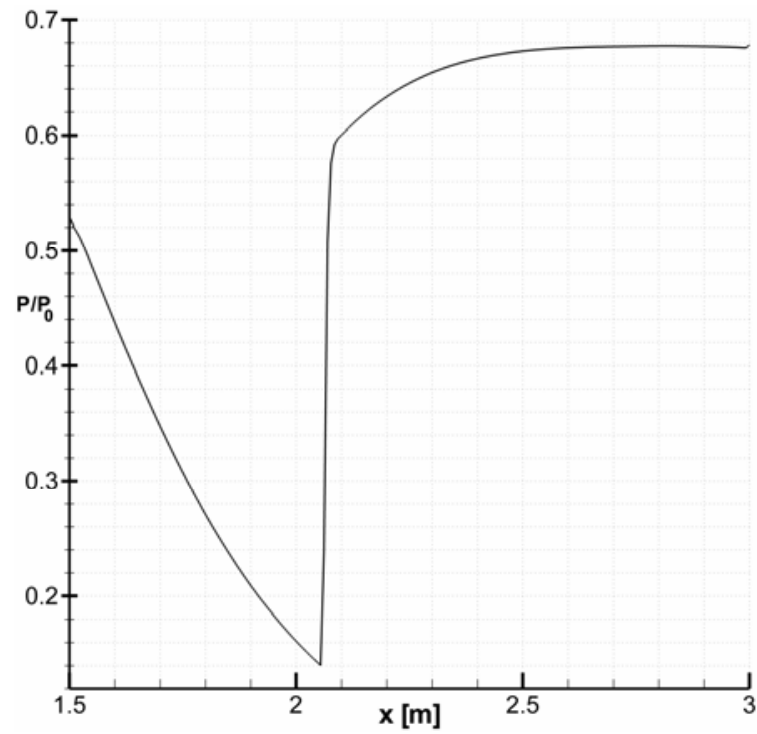

(b)

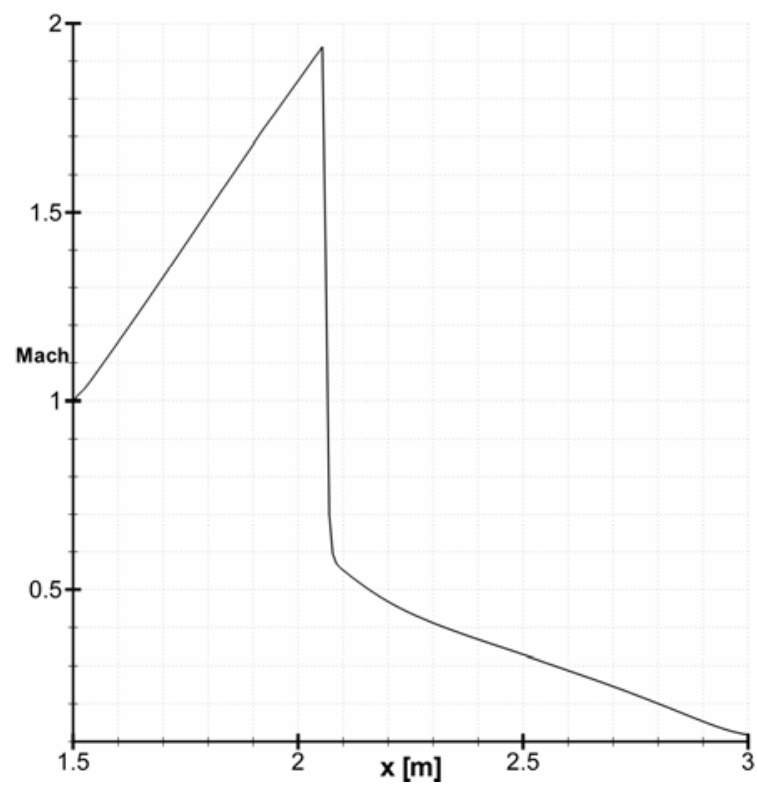

(d)

Figura 7.7 (a) Comportamento da pressão versus número de iterações para um nó fixo da malha. Os seguintes gráficos: (b) Pressão, (c) Temperatura e (d) Número Mach atingiram o regime permanente e indicam sua distribuição espacial ao longo de uma linha fixa da malha.

\subsubsection{Bocal supersônico com vetor velocidade oblíquo}

\section{- Descrição do problema}

O bocal supersônico com vetor velocidade oblíquo é aquele cujo vetor velocidade na entrada apresenta um ângulo de giro fixo. A geometria deste bocal é a mesma da região 
bifásica dos jatos altamente superaquecidos. Consideraremos o caso axissimétrico, isso significa que o contorno está composto por quatro líneas: Linha inicial, Linha de simetria, Linha ao longe e Linha de parede. A condição do escoamento na entrada é que ele seja sônico, $M=1$, e para este caso não conhecemos a solução analítica. O código computacional $D C D$ $2 D v 1$ solucionou esse fenômeno para o caso axissimétrico 2D e como fluido teste o ar. Na Tabela 7.6 apresentamos as condições de reservatório. A Linha inicial é ligeiramente horizontal e é aquela que está ao lado esquerdo do eixo $x$ na Fig. 7.7a. As condições de contorno são conhecidas na Linha inicial, a saber: $P_{i}=485.999[\mathrm{kPa}]$ e $T_{i}=283.332[\mathrm{~K}]$ em todos seus pontos. Na Linha ao longe onde a pressão é: $P_{\infty}=499.560$ [kPa] .

Tabela 7.6 Bocal supersônico com vetor velocidade oblíquo.

\begin{tabular}{|c|c|c|c|}
\hline Condições de Reservatório: & \multicolumn{2}{|c|}{ Condições de contorno } & \multirow{2}{*}{ Solução numérica } \\
\hline$P_{0}=920[\mathrm{kPa}], \quad T_{0}=340[\mathrm{~K}]$ & Linha inicial & Linha ao longe & \\
\hline Mach & 1.0 & & \\
\hline Temperatura: & 0.8333 & & \\
\hline Pressão: & 0.5283 & & \\
\hline Pressão "Far-Field": $\quad P_{\infty}[\mathrm{kPa}]$ & & 499.560 & \\
\hline Dimensão da onda de choque [mm] & & & $r_{1}=8.89 \quad r_{2}=9.70$ \\
\hline Número de nós da malha & & & $110 \times 239=26290$ \\
\hline Tempo computacional [hh:mm:ss:ms] & & & 03:12:59:890 \\
\hline
\end{tabular}

Na Fig. 7.8a temos a geometria da expansão bifásica de dimensões (16x16) milímetros, com uma malha estruturada obtida por transformações. A malha usada para este teste foi de $(110 \times 239=26290) 26290$ nós. Nas figuras 7.8b, 7.8c e 7.8d são mostradas as distribuições de pressão, velocidades e número de Mach, respectivamente. As dimensões da onda de choque são na direção radial $r_{1}=8.89$ [mm] e na direção axial $r_{2}=9.70$ [mm]. 


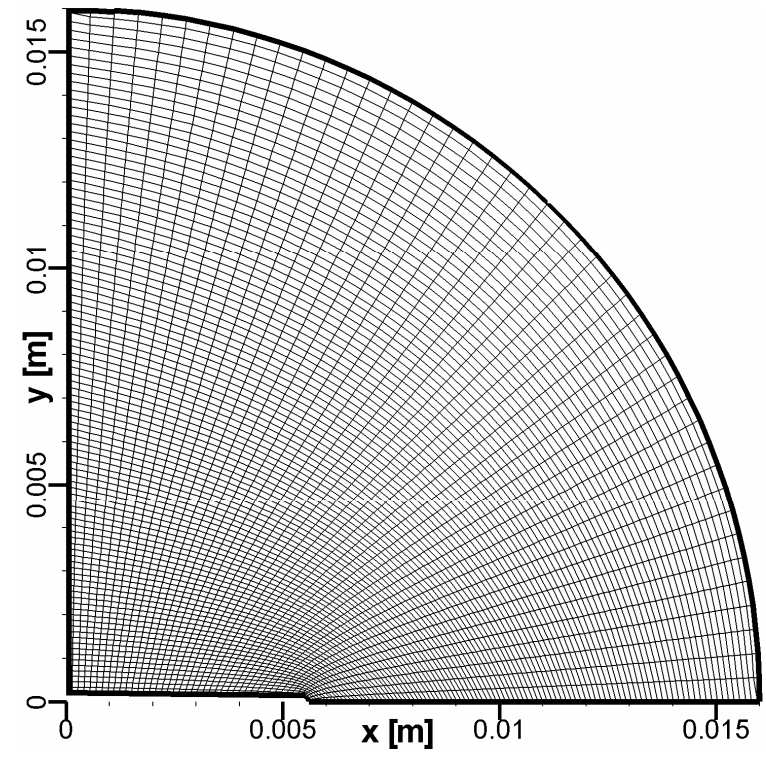

(a)

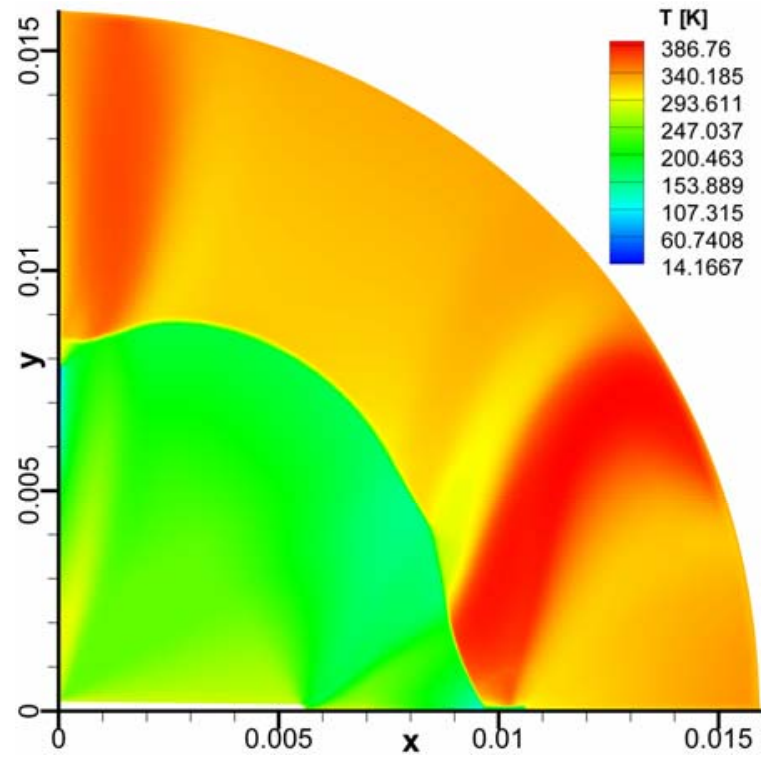

(c)

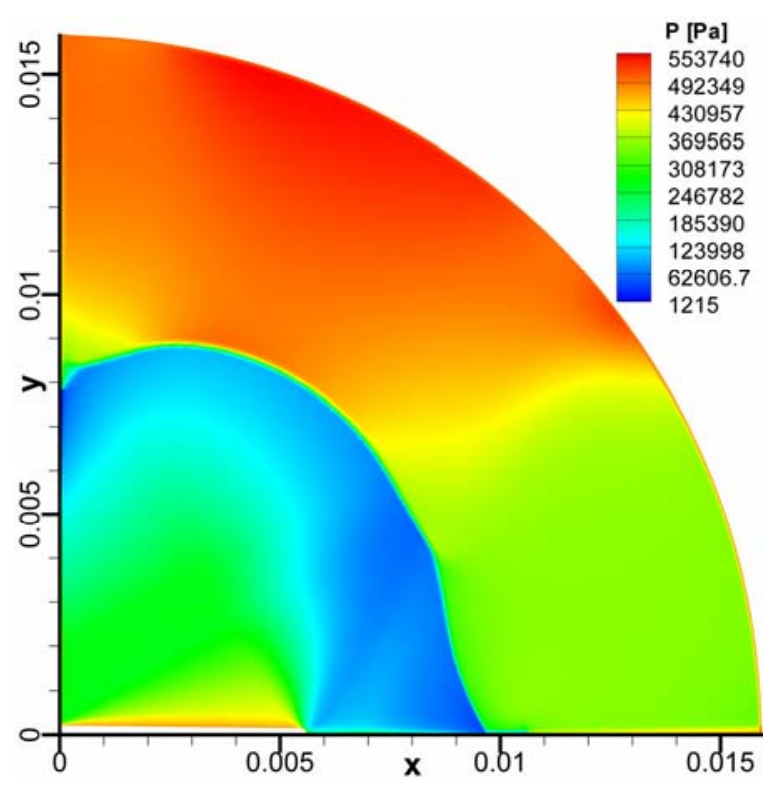

(b)

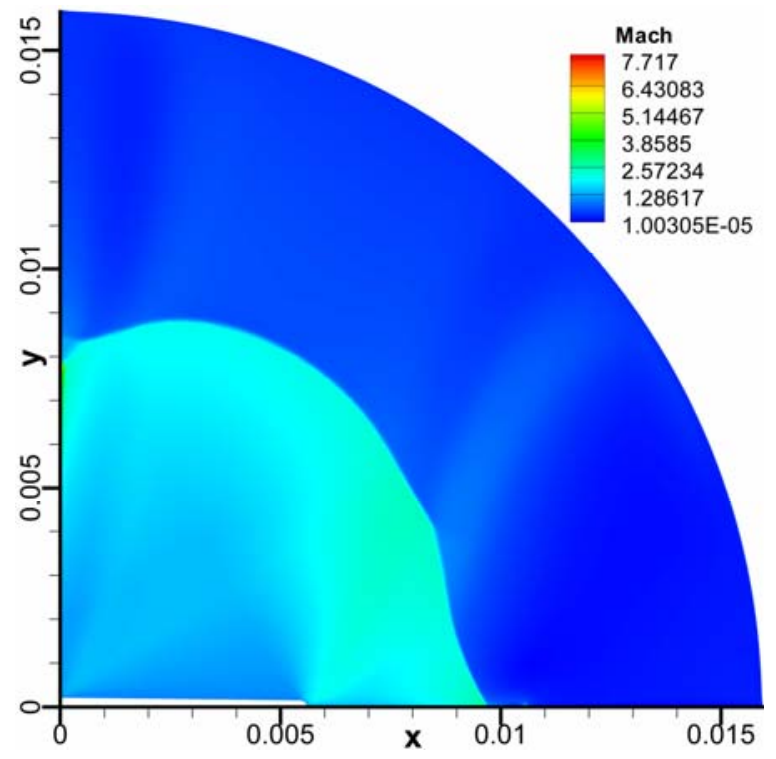

(d)

Figura 7.8 Jato evaporativo. (a) Geometria com malha. (b) Distribuição de pressões. (c) Distribuição de temperaturas. (d) Número Mach.

O problema do jato evaporativo atingiu o estado de regime permanente após 18000 iterações em 3hs, 12m, 59s e 890 milisegundos de tempo CPU. Na Fig. 7.9a confirmamos o regime permanente da pressão que assume o valor de $480[\mathrm{kPa}]$ para um ponto arbitrário: $(55,215)$ da malha. Nas figuras 7.9b e 7.9c mostram-se os perfis das relações de pressões e temperaturas e na Fig. 7.9d temos a curva do número de Mach. Estes perfis foram obtidos ao longo da linha da malha $(i=55 ; j=\overline{0,238})$. 


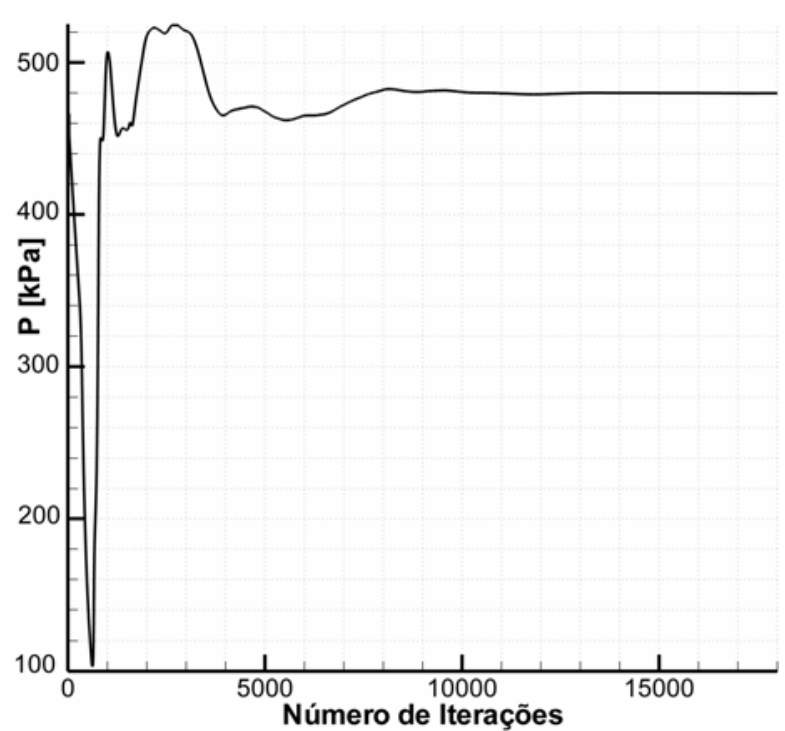

(a)

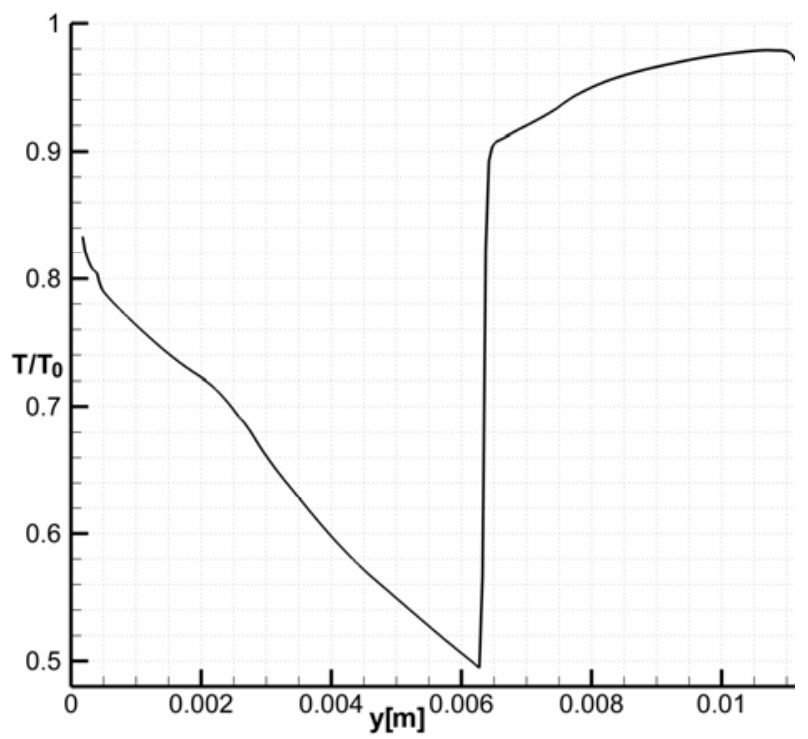

(c)

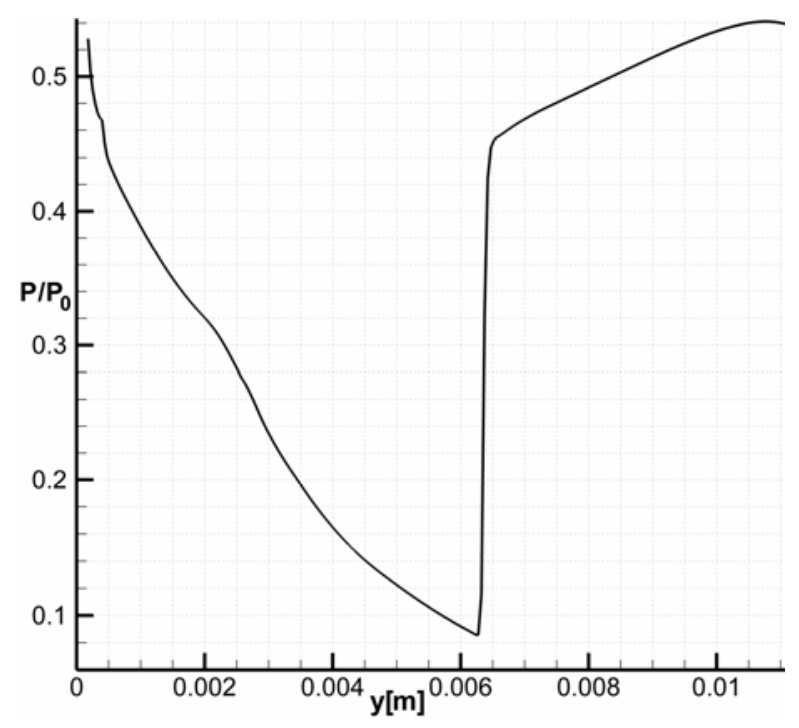

(b)

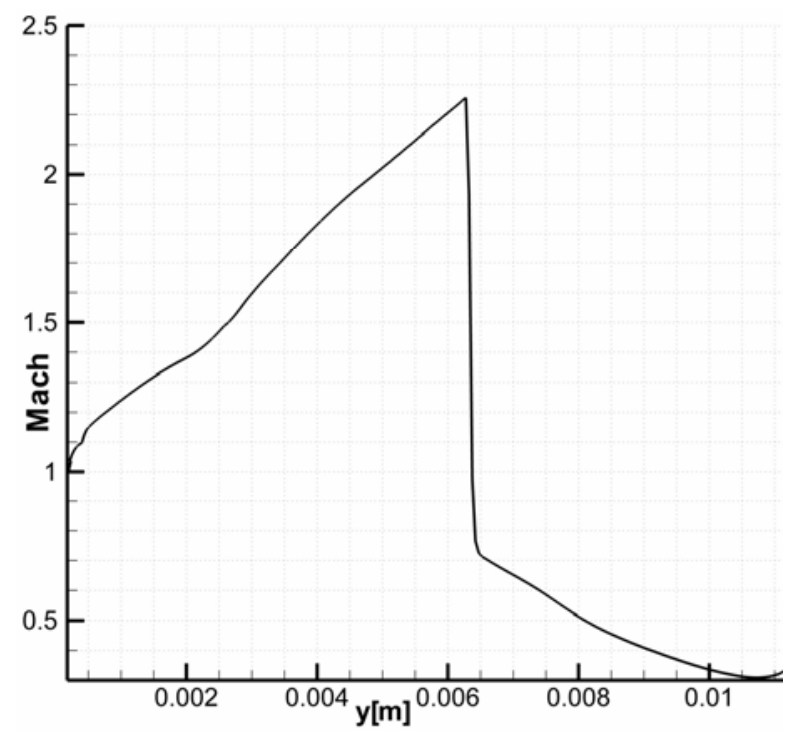

(d)

Figura 7.9 (a) Comportamento da pressão versus número de iterações para um nó fixo da malha. Os seguintes gráficos: (b) Pressão, (c) Temperatura e (d) Número Mach atingiram o regime permanente e indicam sua distribuição espacial ao longo de uma linha fixa da malha.

\subsection{Expansão Bifásica}

O código computacional $D C D-2 D$ v1 foi aplicado desta vez para solucionar numericamente o fenômeno de evaporação rápida em jatos evaporativos de líquidos metaestáveis axissimétrico 2D bifásico. O fluido teste foi o iso-octano $\left(C_{8} H_{18}\right)$ e a equação de estado realística usada foi a tabela gerada a partir da equação Lee-Kesler. Serão estudados 
dois testes o Teste 11 e o Teste 04 solucionados experimentalmente, (VIEIRA, 2005) e numericamente neste trabalho, assim como mostra a Tabela 6.2.

A região bifásica $2 \mathrm{D}$ utilizada é de raio $R_{f}=22[\mathrm{~mm}]$, isto é: $22[\mathrm{~mm}]$ na direção axial e 22 [mm] na direção radial.

A área superficial do cone evaporativo se chamará de área inicial e denotaremos por: $A_{0}$ e a área superficial da semi-esfera que contem a expansão bifásica chamaremos de área ao longe e denotaremos por $A_{\infty}$. A área ao longe para estes dois testes será fixa e toma o valor de: $A_{\infty}=2.981424 \mathrm{E}-003\left[\mathrm{~m}^{2}\right]$

As malhas usadas de raio $R_{f}=22[\mathrm{~mm}]$ são de dimensão $(M \times N)$ nós, e foram considerados cinco tipos diferentes de malhas refinadas, a saber: $\boldsymbol{A}, \boldsymbol{B}, \boldsymbol{C}, \boldsymbol{D}$ e $\boldsymbol{E}$, assim como se mostra na Tabela 7.7. Nenhuma malha teve um tipo especial de refinamento local, assim como se fez no Cap. (4.6). Os refinamentos foram em toda a geometria do domínio. Por outro lado, é importante lembrar que a Linha inicial é a união de uma linha reta com uma linha curva que corresponde à ponta do cone evaporativo (objetivo de suavizar a condição de contorno).

Tabela 7.7 Grupo de malhas refinadas, $R_{f}=22[\mathrm{~mm}]$.

\begin{tabular}{c|c|c|c|c|c}
\hline \multirow{2}{*}{$\begin{array}{c}\text { Tipo de } \\
\text { Malhas } \\
R_{f}=22[\mathrm{~mm}]\end{array}$} & \multicolumn{3}{|c|}{ Linha Inicial } & Parede & Total de nós \\
\cline { 2 - 5 } & Linha reta & Linha curva & $M$ & $N$ & $M \times N$ \\
\hline A & 70 & 7 & 77 & 161 & 12397 \\
B & 80 & 8 & 88 & 184 & 16192 \\
C & 90 & 9 & 99 & 207 & 20493 \\
D & 100 & 10 & 110 & 230 & 25300 \\
E & 110 & 11 & 121 & 253 & 30613 \\
\hline
\end{tabular}

As condições de contorno obedecem às estudadas no Apêndice B. No referente à geometria do contorno do domínio bifásico, como já tratadas no Cap. (3.3), ele é composto pela Linha inicial, Linha ao longe, Linha de parede e Linha de simetria. As condições de contorno para a Linha inicial são todas conhecidas e são fornecidas pela Tabela 6.1. As condições de contorno para a Linha ao longe não são conhecidas, unicamente impomos a condição da pressão “Far-Field”. A única condição de contorno para a Linha de parede é que a componente do vetor velocidade perpendicular à parede deve ser nula. A única condição de 
“contorno” para a Linha de simetria é que a componente do vetor velocidade perpendicular à simetria deve ser nula.

\subsubsection{Teste 11}

As condições de reservatório, condições de contorno e os resultados numéricos são mostrados na Tabela 7.8.

A área inicial é $A_{0}=6.351266 \mathrm{E}-006\left[\mathrm{~m}^{2}\right]$.

Tabela 7.8 Solução numérica do Teste 11.

\begin{tabular}{|c|c|c|c|c|c|}
\hline \multicolumn{2}{|c|}{$\begin{array}{c}\text { TESTE } 11 \\
\text { Condições de Reservatório: }\end{array}$} & \multirow{2}{*}{$\begin{array}{l}\text { Dados } \\
\text { geométricos }\end{array}$} & \multicolumn{2}{|c|}{ Condições de contorno } & \multirow{2}{*}{$\begin{array}{l}\text { Solução } \\
\text { numérica }\end{array}$} \\
\hline$P_{0}=503.4[\mathrm{kPa}]$ & $T_{0}=368.55[\mathrm{~K}]$ & & Linha inicial & Linha ao longe & \\
\hline Mach & & \multirow{9}{*}{$\begin{array}{c}C \\
469.42\end{array}$} & 1.0 & \multirow{9}{*}{8.0} & \\
\hline Temperatura: & $T[\mathrm{~K}]$ & & 331.40 & & \\
\hline Pressão: & $P[\mathrm{kPa}]$ & & 26.862 & & \\
\hline Título: & $x$ & & 0.2965 & & \\
\hline Pressão "Far-Field": & $P_{\infty}[\mathrm{kPa}]$ & & & & \\
\hline Tipo de malha & & & & & \\
\hline Relação de áreas: & $A_{\infty} / A_{0}$ & & & & \\
\hline \multicolumn{2}{|c|}{ Dimensão da onda de choque [mm] } & & & & $\begin{array}{l}r_{1}=14.84 \\
r_{2}=15.32\end{array}$ \\
\hline Tempo CPU & [hh:mm:ss:ms] & & & & 04:33:46:859 \\
\hline
\end{tabular}

A malha que se usa para solucionar o problema é do tipo C, na Fig. 7.10a temos a malha C só que com menos linhas por questão de visualização. Nas seguintes figuras 7.10b, 7.10c, 7.10d, 7.10e e 7.10f apresentamos a distribuição de pressão, temperatura, número de Mach, título e densidade, respectivamente. Observe que nas extremidades da Linha inicial a expansão é rápida, enquanto na lateral da mesma, a expansão é lenta formando um cone com vértice na entrada do bocal. 

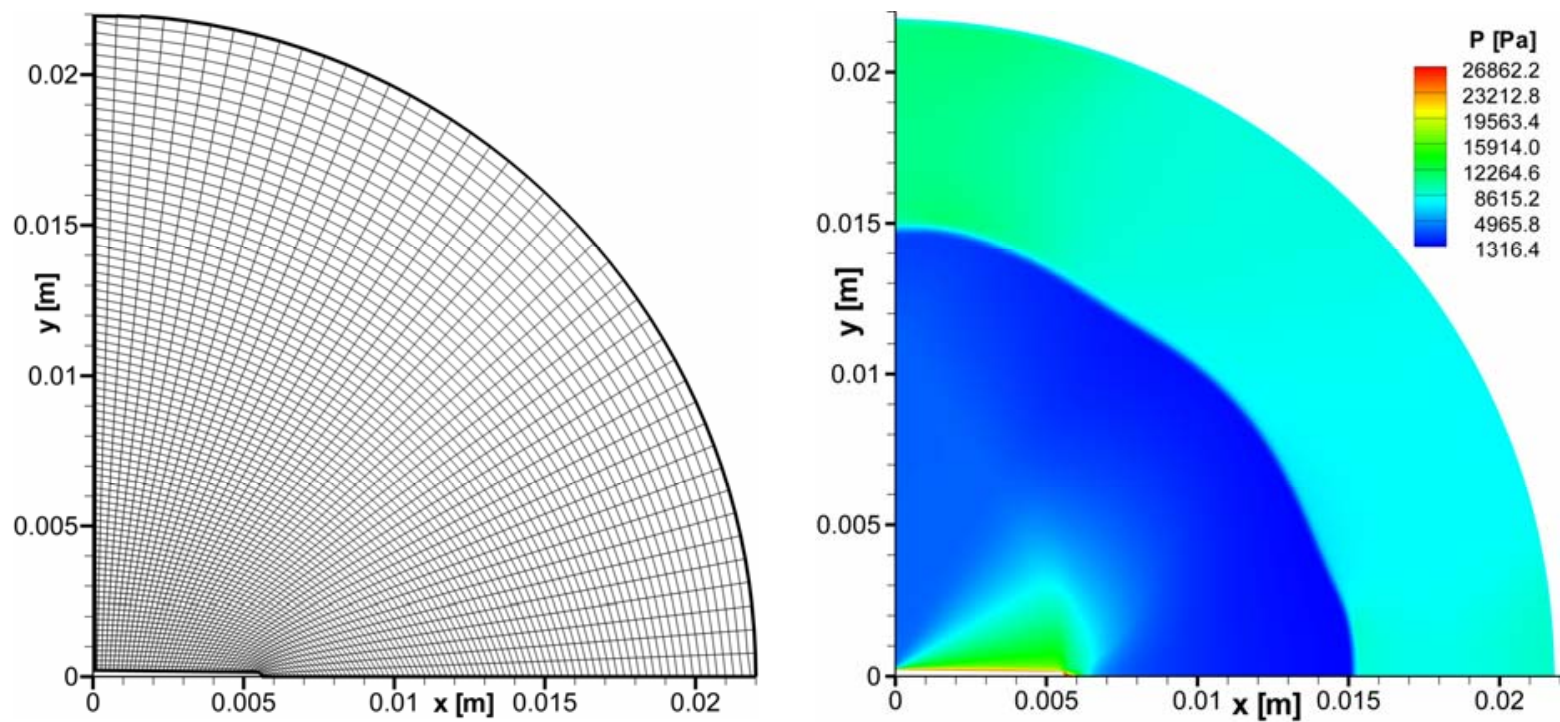

(a)
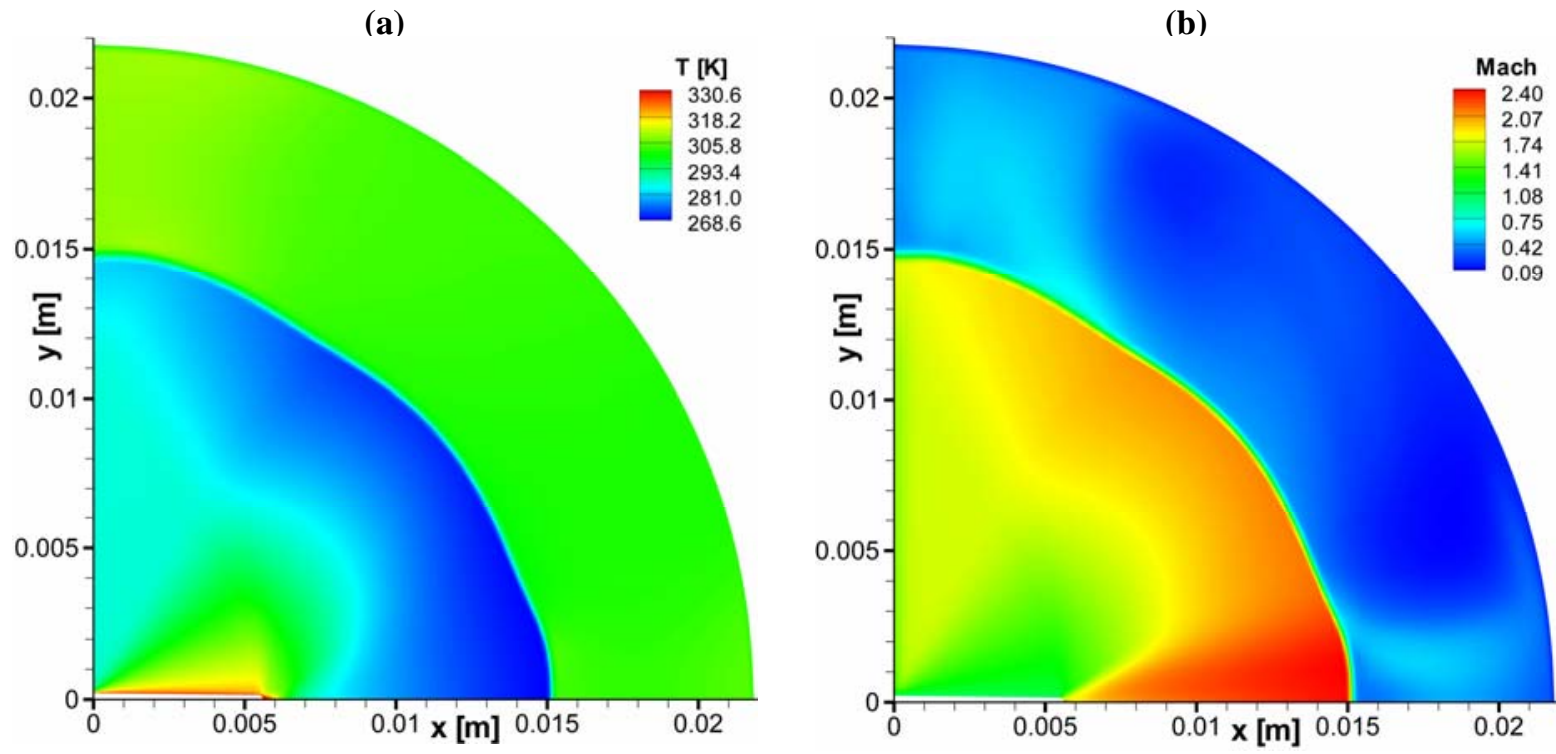

(c)

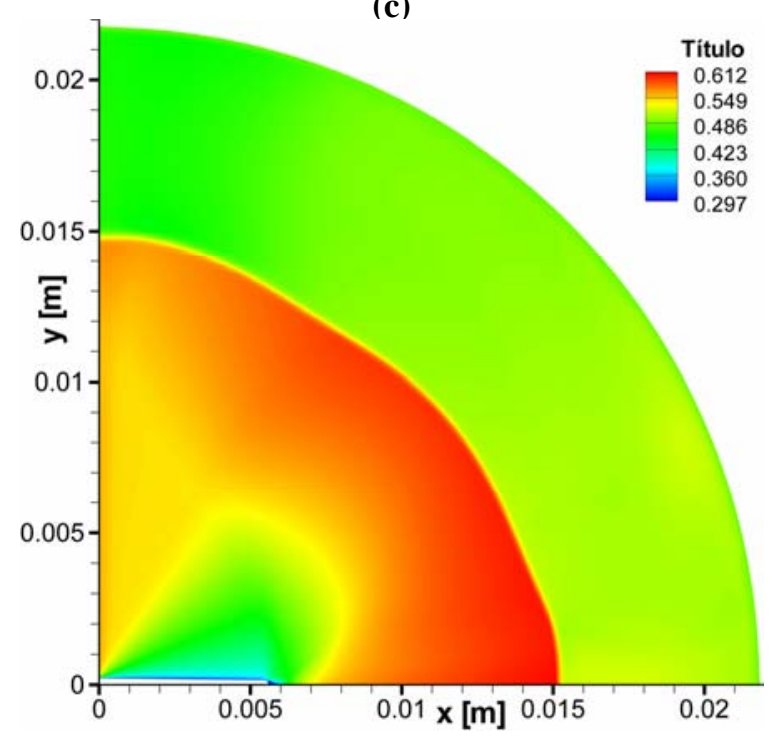

(d)

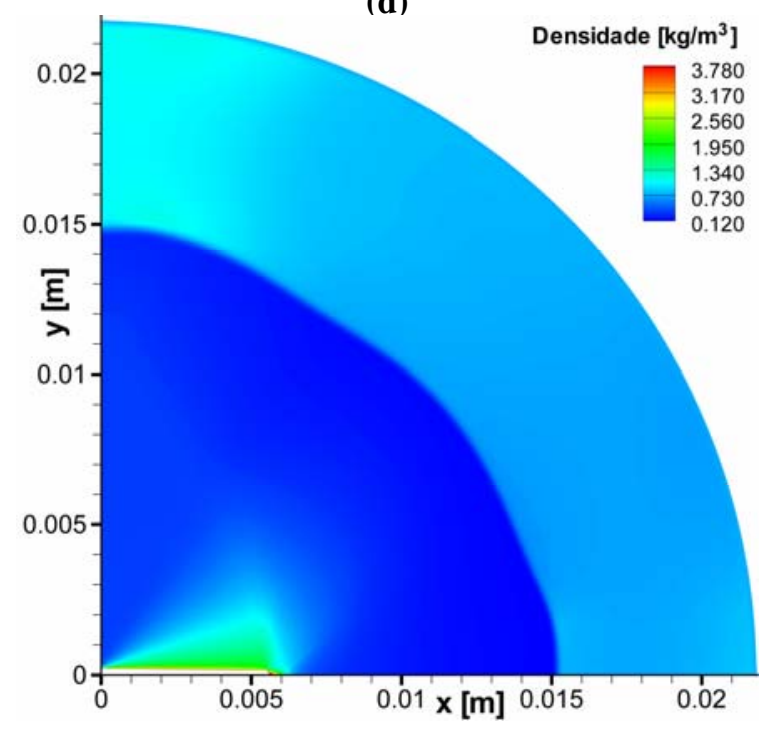

(e)

(f)

Figura 7.10 Jato supersônico. (a) Geometria com malha. (b) Distribuição de pressões. (c) Distribuição de temperaturas. (d) Número Mach. (e) Título. (f) Densidade. 
Na figura 7.11a temos o estado permanente para o ponto $(50,184)$ da malha. Vemos que o estado de regime permanente atingiu após 14000 iterações, num tempo computacional de 4 h., 33 m., 46 s. e 859 milisegundos. A pressão nesse ponto toma o valor de 8160.87 [Pa]. Nas próximas figuras 7.11b, 7.11c e 7.11d temos os perfis da pressão, número de Mach e título, respectivamente. Estes perfis estão sobre a linha $\left(i_{0}=49 ; j=\overline{0,206}\right)$ da malha.
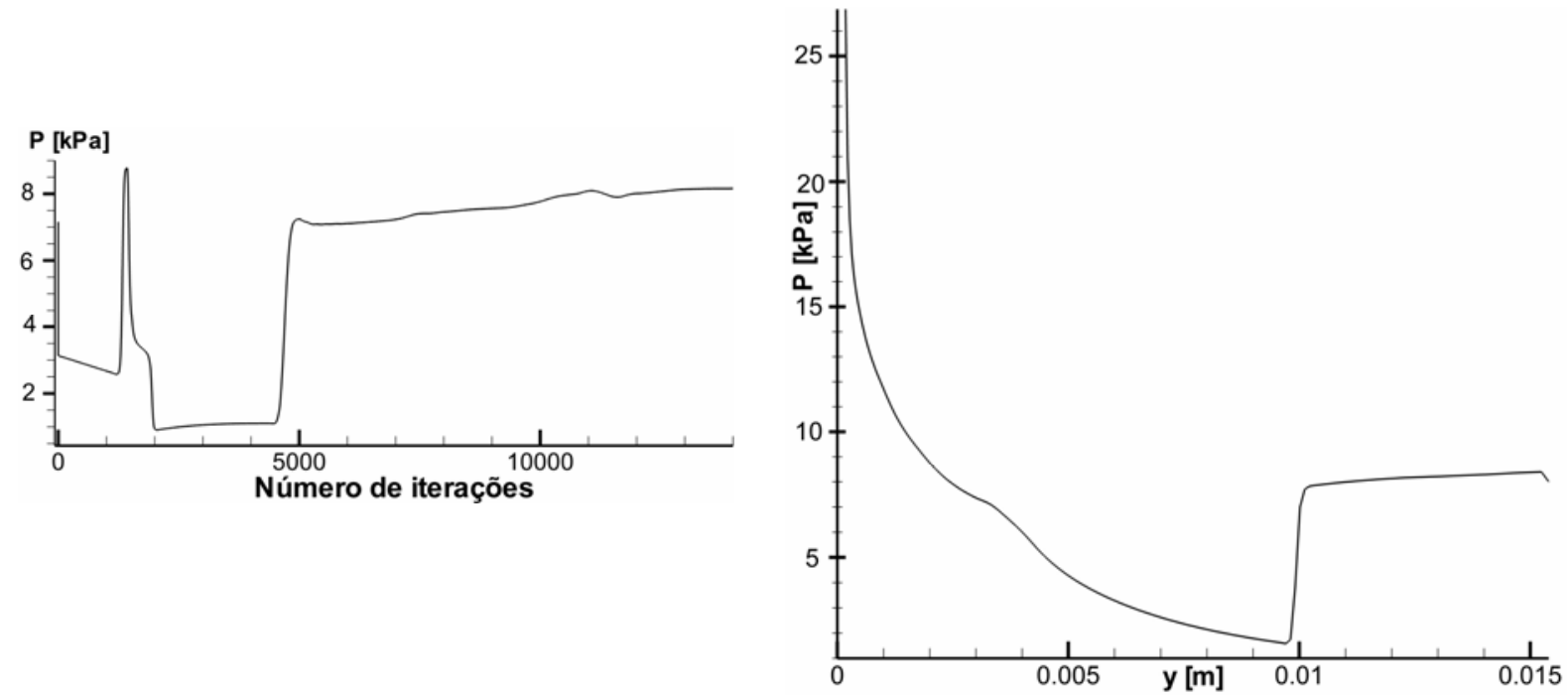

(a)

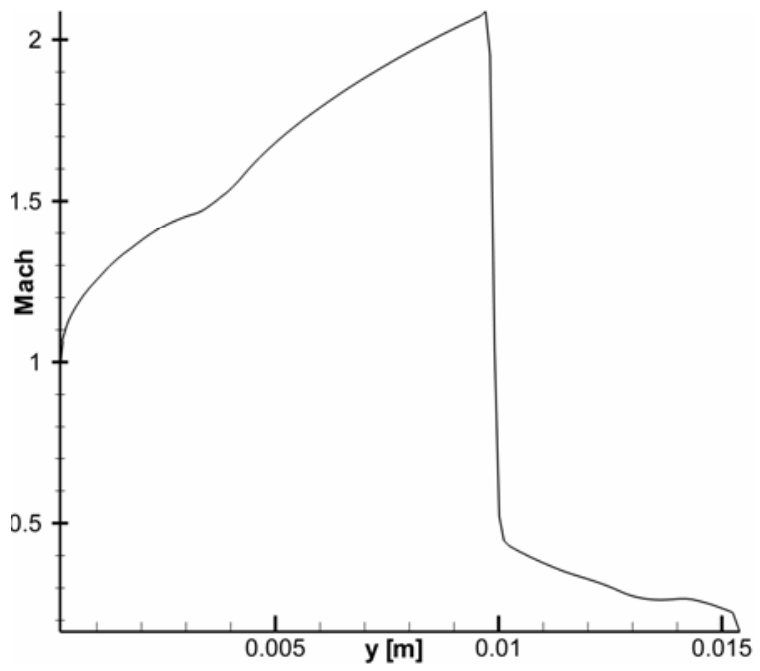

(c) (b)

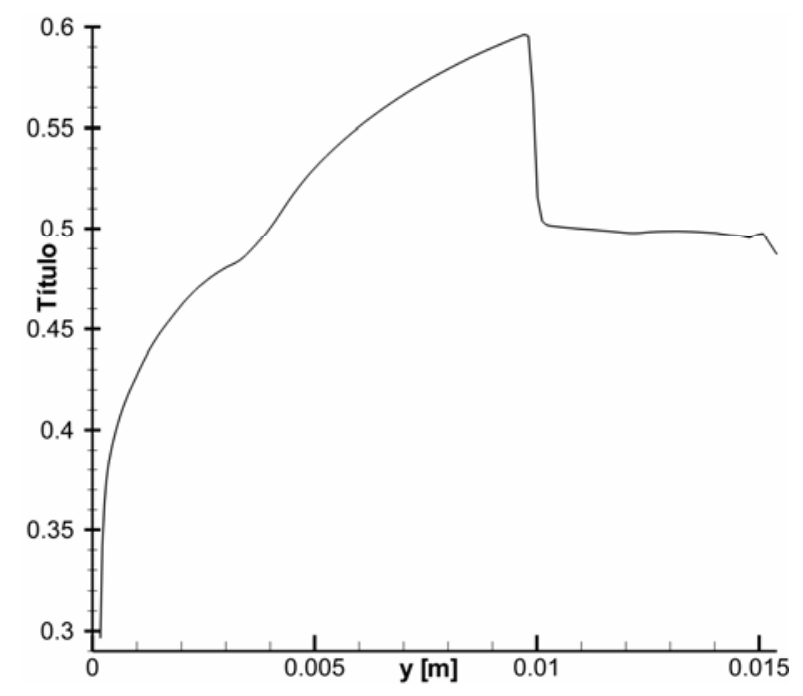

(d)

Figura 7.11 (a) Comportamento da pressão versus número de iterações para um nó fixo da malha. Os seguintes gráficos: (b) Pressão, (c) Número Mach e (d) Título atingiram o regime permanente e indicam sua distribuição espacial ao longo de uma linha fixa da malha. 
Para visualizar melhor a distribuição de pressões e a formação da onda de choque veja o gráfico de pressões em 3D na Fig. 7.12.

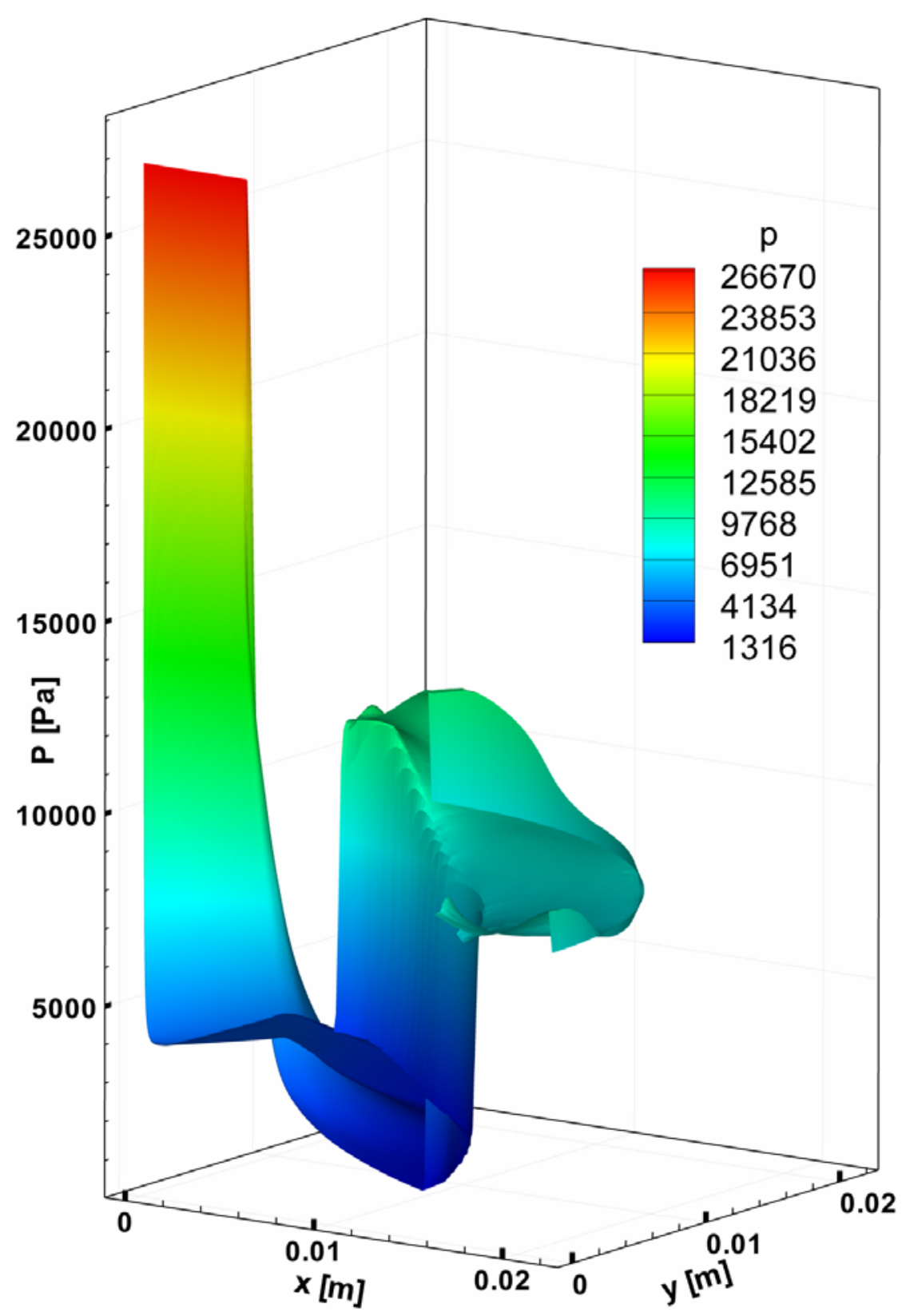

Figura 7.12 Distribuição do gráfico da pressão em 3D.

O código $D C D-2 D$ v1 também resolve problemas sem simetria axial. Para ter uma idéia mais clara da expansão bifásica se resolveu o problema não-simétrico, veja as condições de reservatório, dados geométricos, condições de contorno e solução numérica na Tabela 7.9. 
Tabela 7.9 Solução numérica para o caso sem condição de simetria.

\begin{tabular}{|c|c|c|c|c|c|}
\hline \multicolumn{2}{|c|}{$\begin{array}{c}\text { TESTE } 11 \\
\text { Condições de Reservatório: }\end{array}$} & \multirow{2}{*}{$\begin{array}{c}\text { Dados } \\
\text { geométricos }\end{array}$} & \multicolumn{2}{|c|}{ Condições de contorno } & \multirow{2}{*}{$\begin{array}{l}\text { Solução } \\
\text { numérica }\end{array}$} \\
\hline \multicolumn{2}{|c|}{$P_{0}=503.4[\mathrm{kPa}], \quad T_{0}=368.55[\mathrm{~K}]$} & & Linha inicial & Linha ao longe & \\
\hline Mach & & & 1.0 & & \\
\hline Temperatura & $T[\mathrm{~K}]$ & & 331.40 & & \\
\hline Pressão & $P[\mathrm{kPa}]$ & & 26.862 & & \\
\hline Título & $x$ & & 0.2965 & & \\
\hline Pressão "Far-Field" & $P_{\infty}[\mathrm{kPa}]$ & & & 10.0 & \\
\hline Número de nós da $n$ & malha & $153 \times 161=24633$ & & & \\
\hline Relação de áreas & $A_{\infty} / A_{0}$ & 469.42 & & & \\
\hline Dimensão da onda c & de choque $[\mathrm{mm}]$ & & & & $\begin{array}{l}r_{1}=23.24 \\
r_{2}=12.75\end{array}$ \\
\hline Tempo CPU & [hh:mm:ss:ms] & & & & 06:23:46:640 \\
\hline
\end{tabular}

A expansão bifásica do campo de pressões e do número Mach, também pode ser observada em seu domínio todo, ou seja, sem simetria axial, veja Fig. 7.13a e 7.13b.

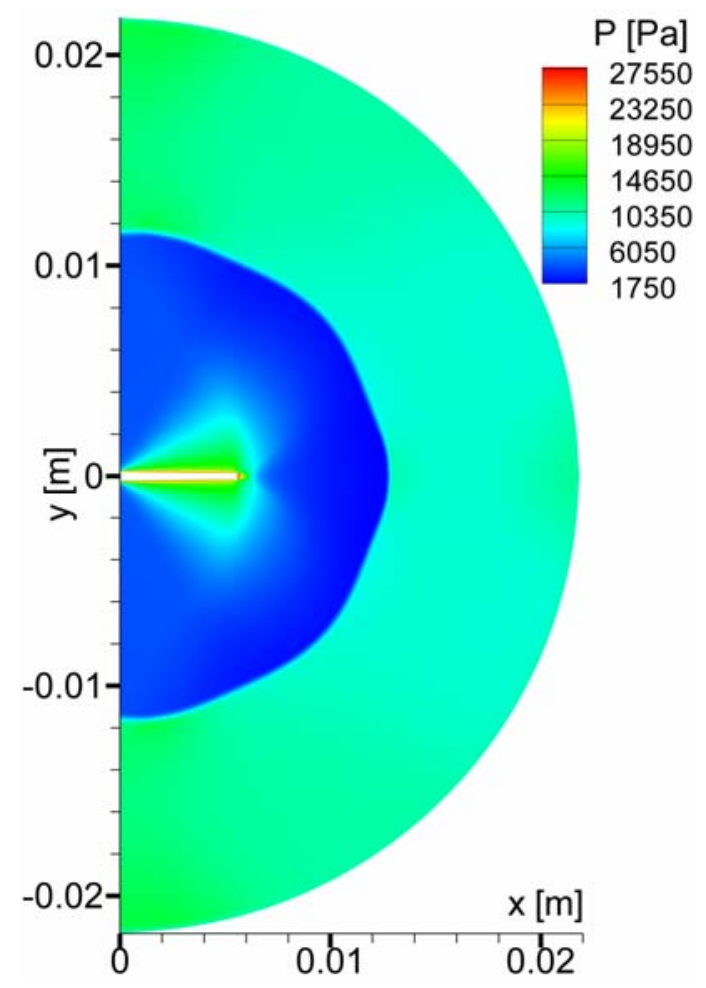

(a)

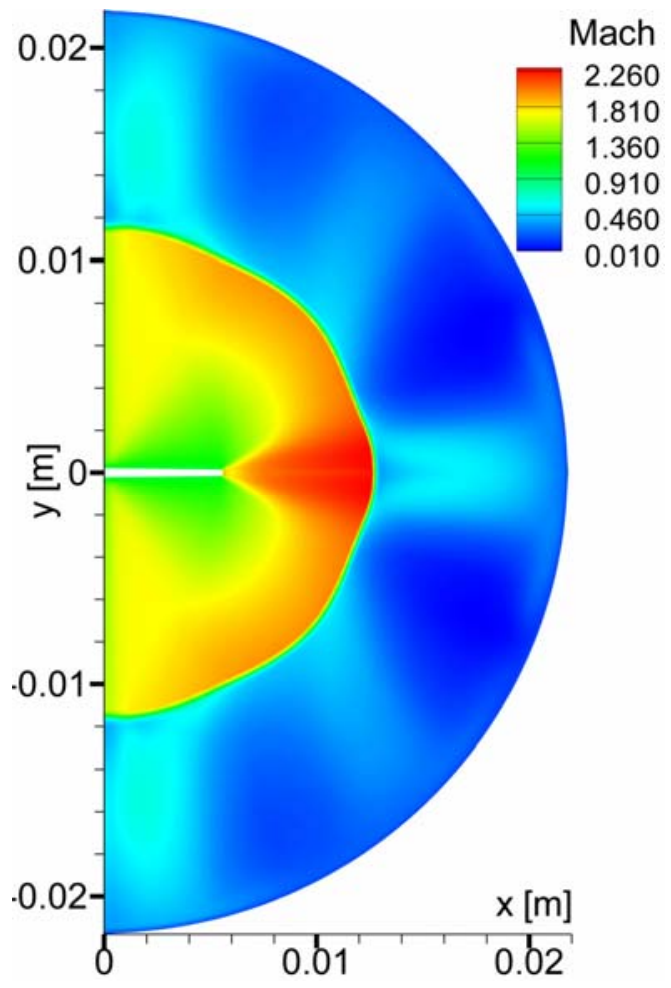

(b)

Figura 7.13 Domínio não-simétrico. a) Distribuição da pressão b) Distribuição do número Mach. 


\subsubsection{Teste 4}

As condições de reservatório, condições de contorno e os resultados numéricos são mostrados na Tabela 7.10.

A área inicial é $A_{0}=1.631501 \mathrm{E}-005\left[\mathrm{~m}^{2}\right]$.

A malha que se usa para solucionar o problema é do tipo C, na Fig. 7.14a temos a malha C só que com menos linhas. Nas seguintes figuras 7.14b, 7.14c, 7.14d, 7.14e e 7.14f apresentamos a distribuição de pressão, temperatura, número de Mach, título e densidade, respectivamente. Observe que nas extremidades da Linha inicial a expansão é rápida, em quanto que na lateral da mesma a expansão é muito lenta formando um cone com vértice na entrada do bocal, este cone é maior que o cone que se forma para o Teste 11.

Tabela 7.10 Solução numérica do Teste 4.

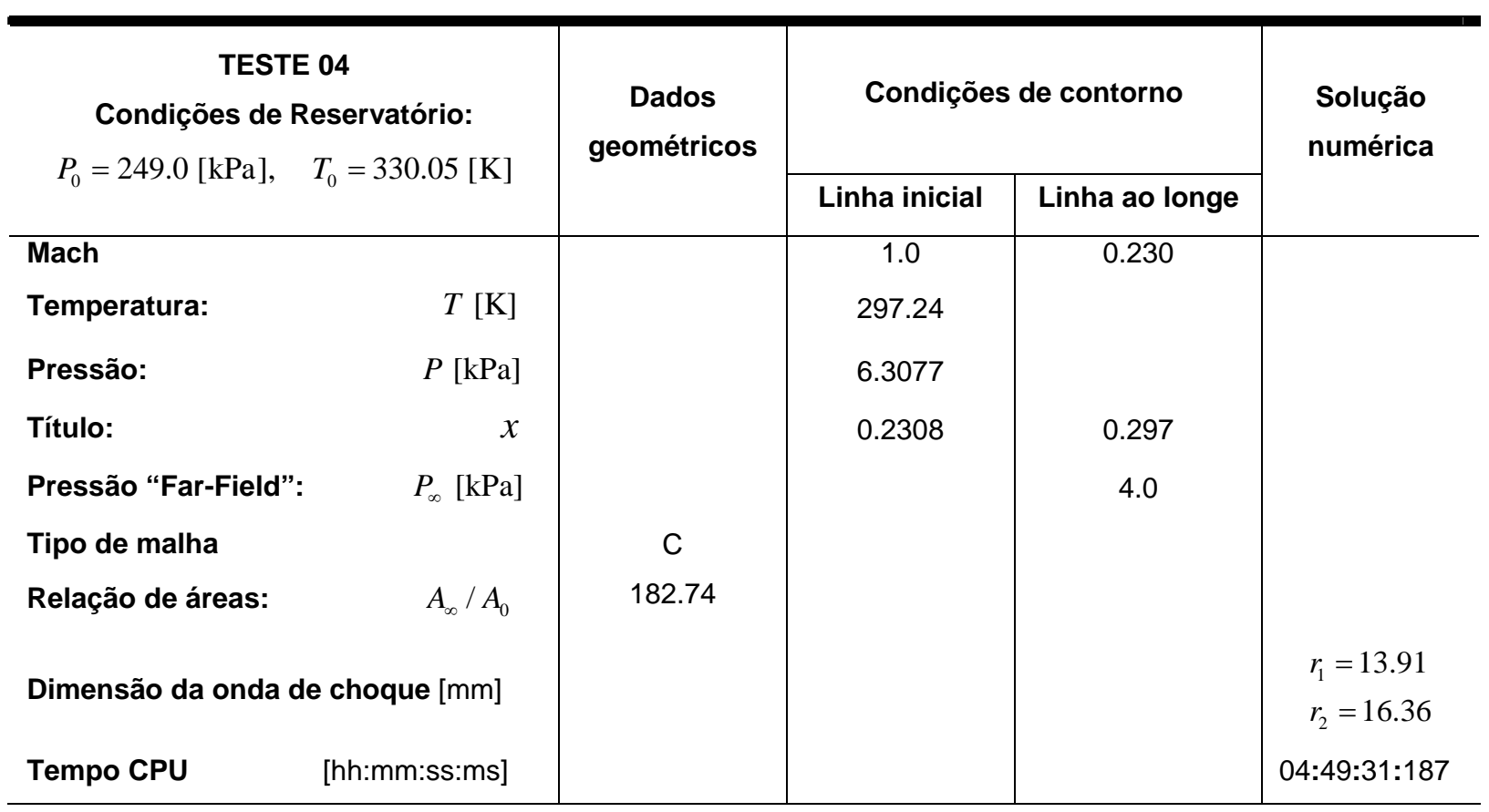




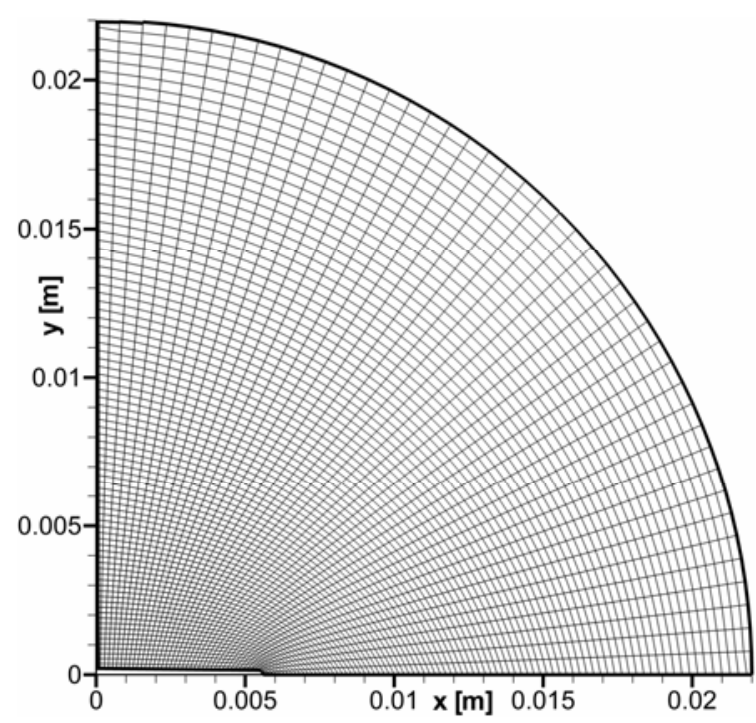

(a)

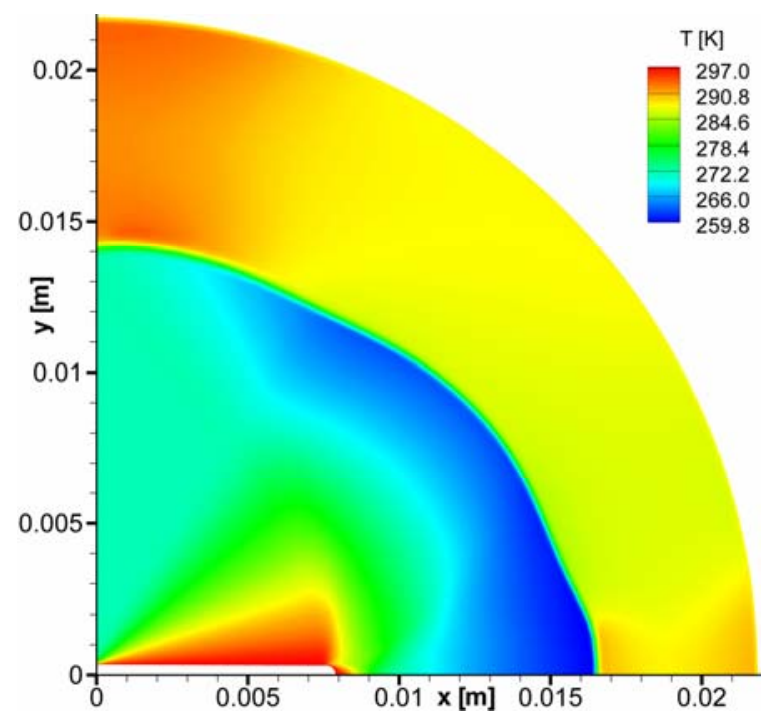

(c)

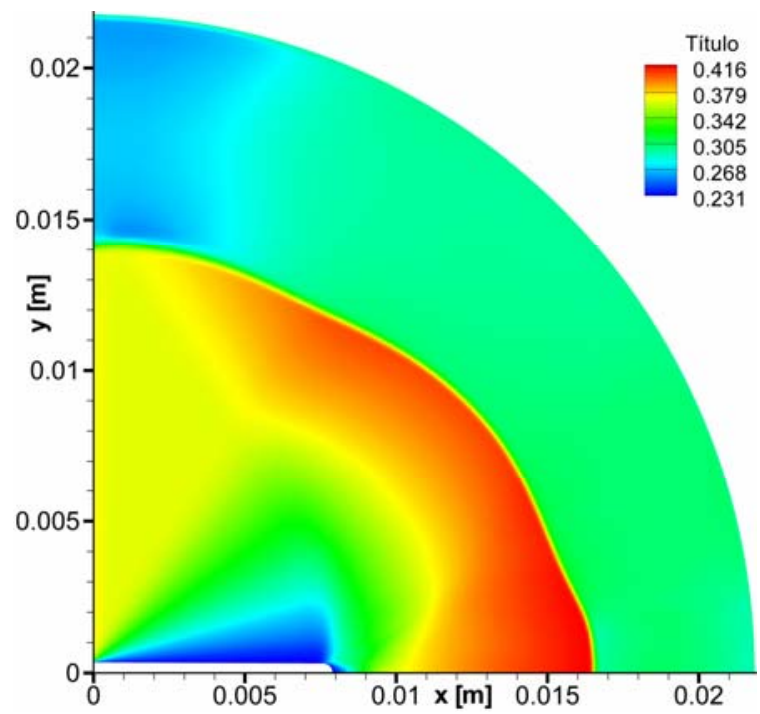

(e)

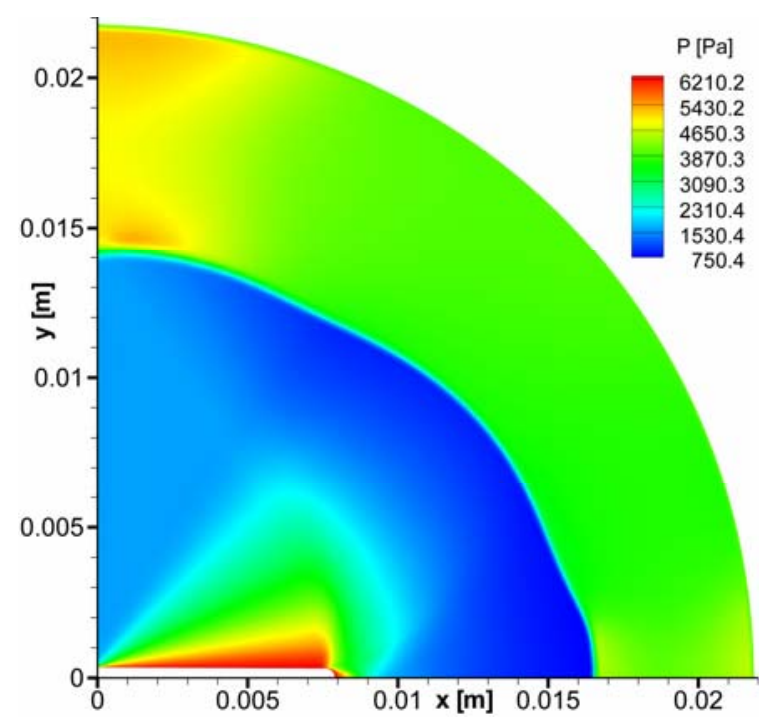

(b)

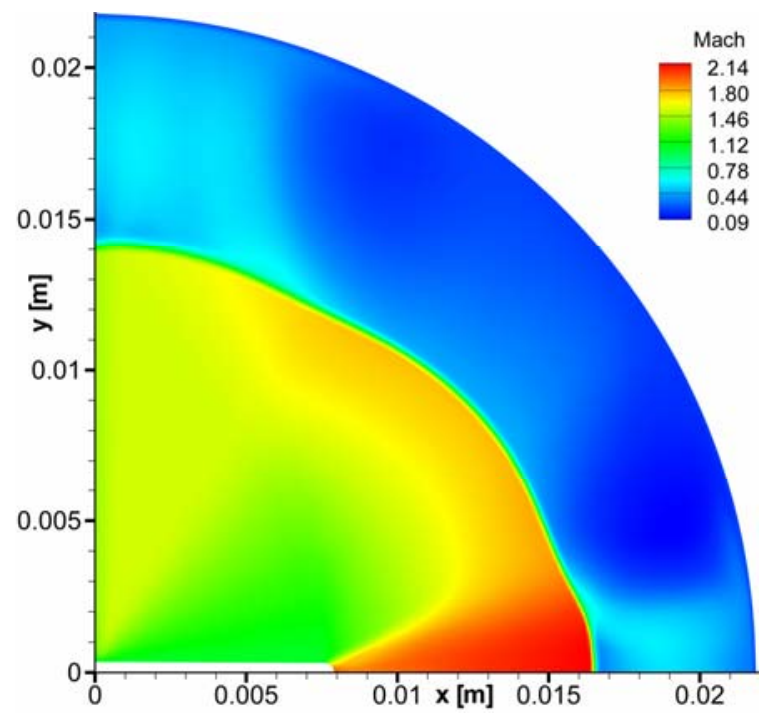

(d)

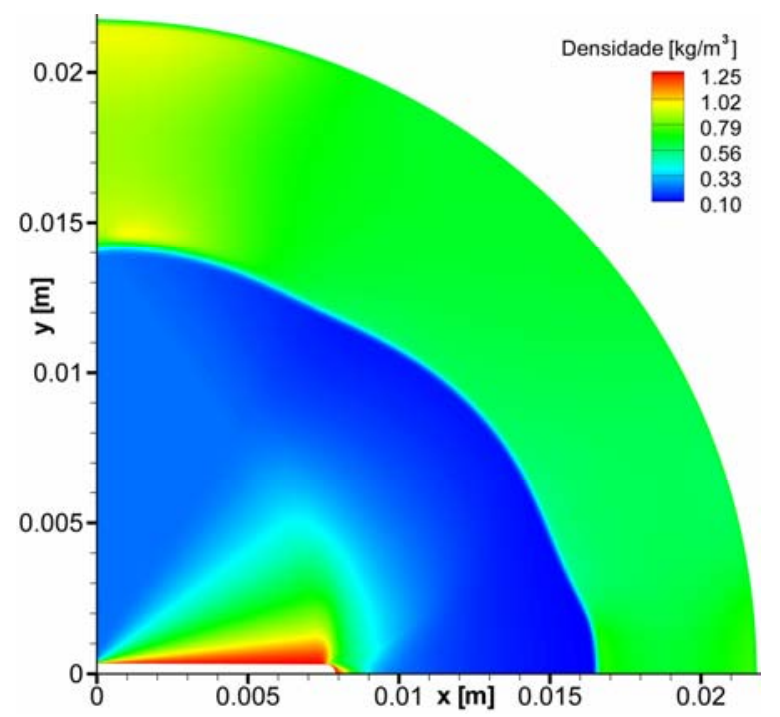

(f)

Figura 7.14 Jato supersônico. (a) Geometria com malha. (b) Distribuição de pressões. (c) Distribuição de temperaturas. (d) Número Mach. (e) Título. (f) Densidade. 
O estado permanente para o ponto $(50,184)$ da malha é após 15000 iterações, num tempo CPU de 4 h., 49 m., 31 s. e 187 milisegundos. A pressão nesse ponto toma o valor de 3870.26 [Pa], veja Fig. 7.15a. Nas próximas figuras 7.15b, 7.15c e 7.15d temos os perfis da pressão, número de Mach e título, respectivamente. Estes perfis estão sobre a linha $\left(i_{0}=49 ; j=\overline{0,206}\right)$ da malha.

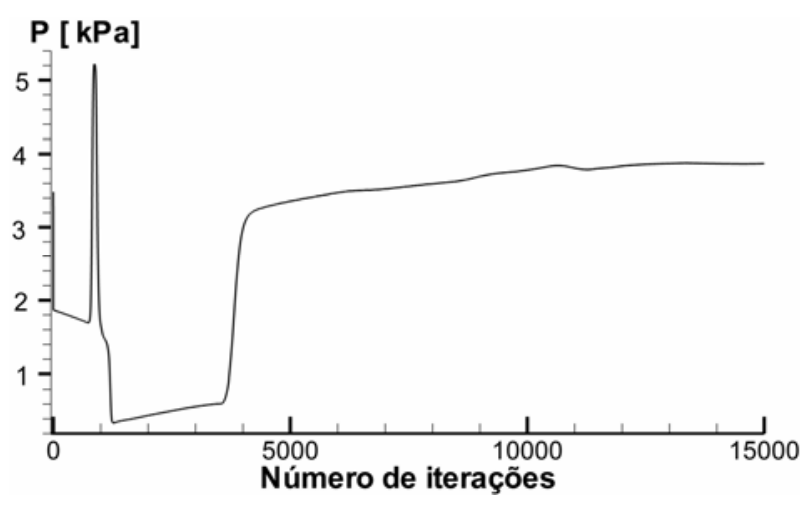

(a)

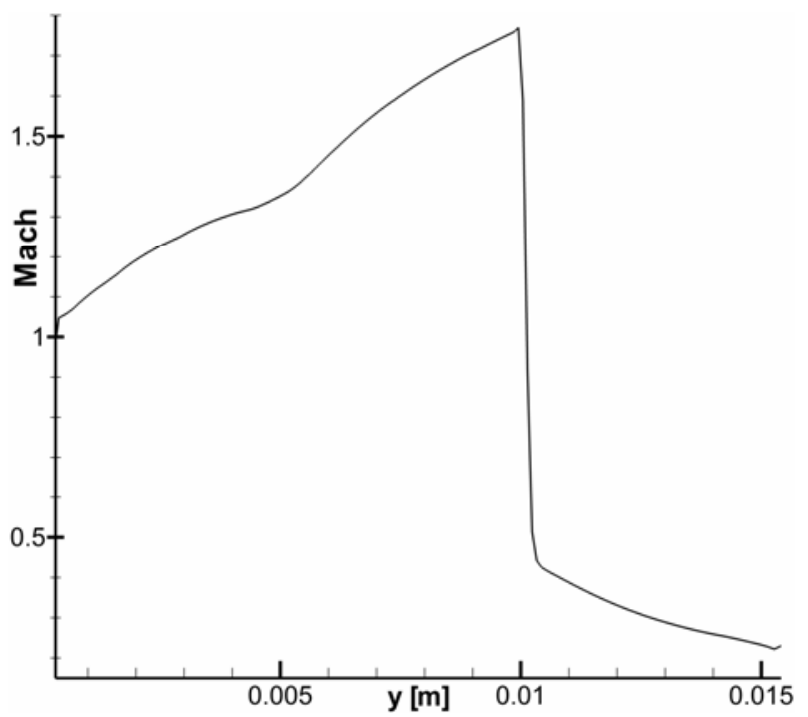

(c)

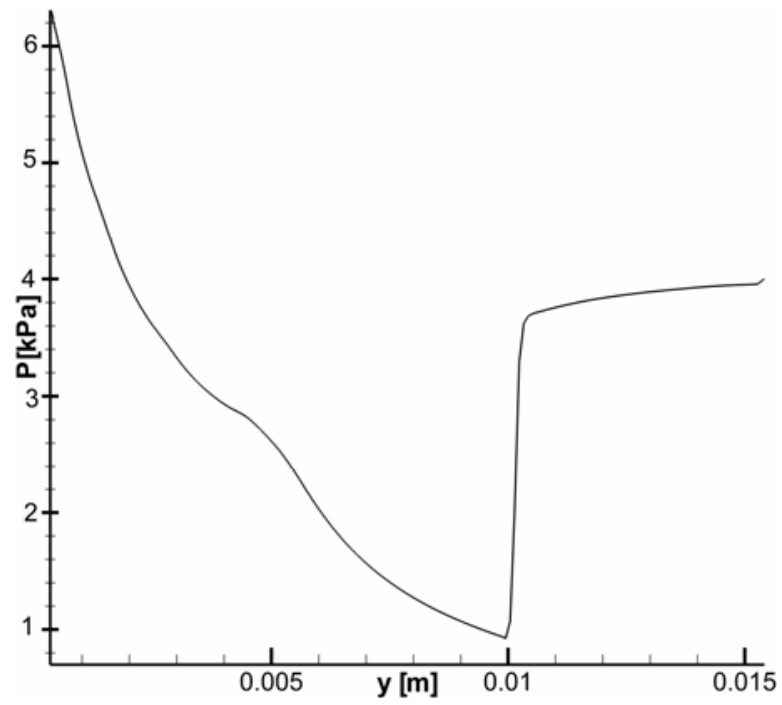

(b)

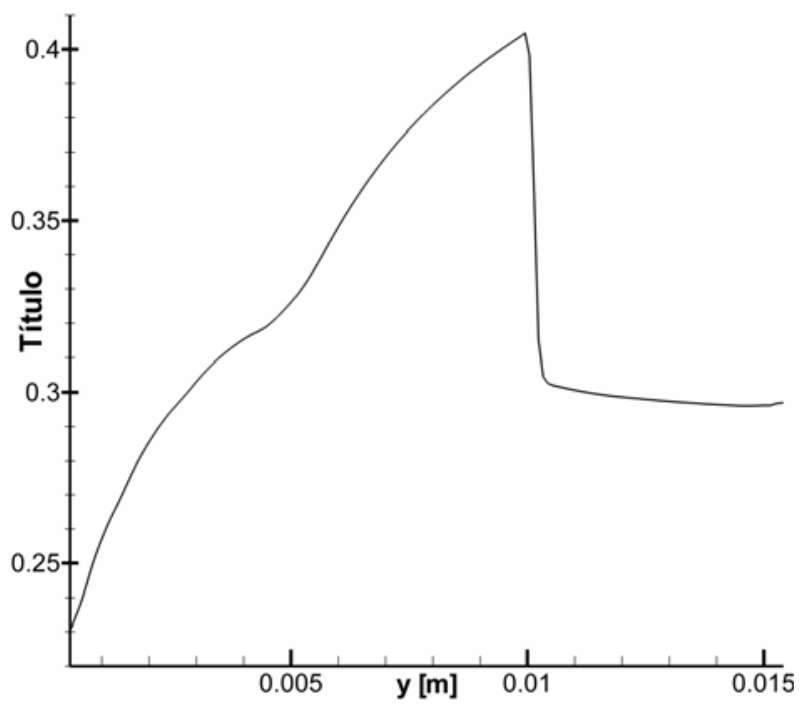

(d)

Figura 7.15 (a) Comportamento temporal da pressão versus número de iterações para um nó fixo da malha. Os seguintes gráficos: (b) Pressão, (c) Número Mach e (d) Título atingiram o regime permanente e indicam sua distribuição espacial ao longo de uma linha fixa da malha. 


\subsubsection{Variação da pressão ao longe}

Agora verificaremos as conseqüências de se variar a condição da pressão ao longe, $P_{\infty}$, na expansão bifásica, as dimensões da onda de choque também variam fato demonstrado por Vieira (2005) e Angelo (2004). Para comprovar esse resultado foi tomado como referência o Teste 11 para uma malha fixa do tipo A e três valores para a pressão ao longe, a saber, 6500, 8000 e 10000 [Pa] tal como se mostra na Tabela 7.11. Pode-se verificar que as dimensões da onda de choque diminuem à medida que a pressão ao longe aumenta, com uma taxa de crescimento maior para o caso de $r_{1}$, assim como se mostra na Fig. 7.16.

Tabela 7.11 Variação da pressão ao longe.

\begin{tabular}{c|c|c|c|c}
\hline \hline Número do Teste & Tipo de malha & $P_{\infty}[\mathrm{Pa}]$ & $r_{1}[\mathrm{~mm}]$ & $r_{2}[\mathrm{~mm}]$ \\
\hline 11 & $\mathrm{~A}$ & 6500 & 17.60 & 17.20 \\
11 & $\mathrm{~A}$ & 8000 & 14.84 & 15.32 \\
11 & $\mathrm{~A}$ & 10000 & 11.68 & 12.90 \\
\hline
\end{tabular}

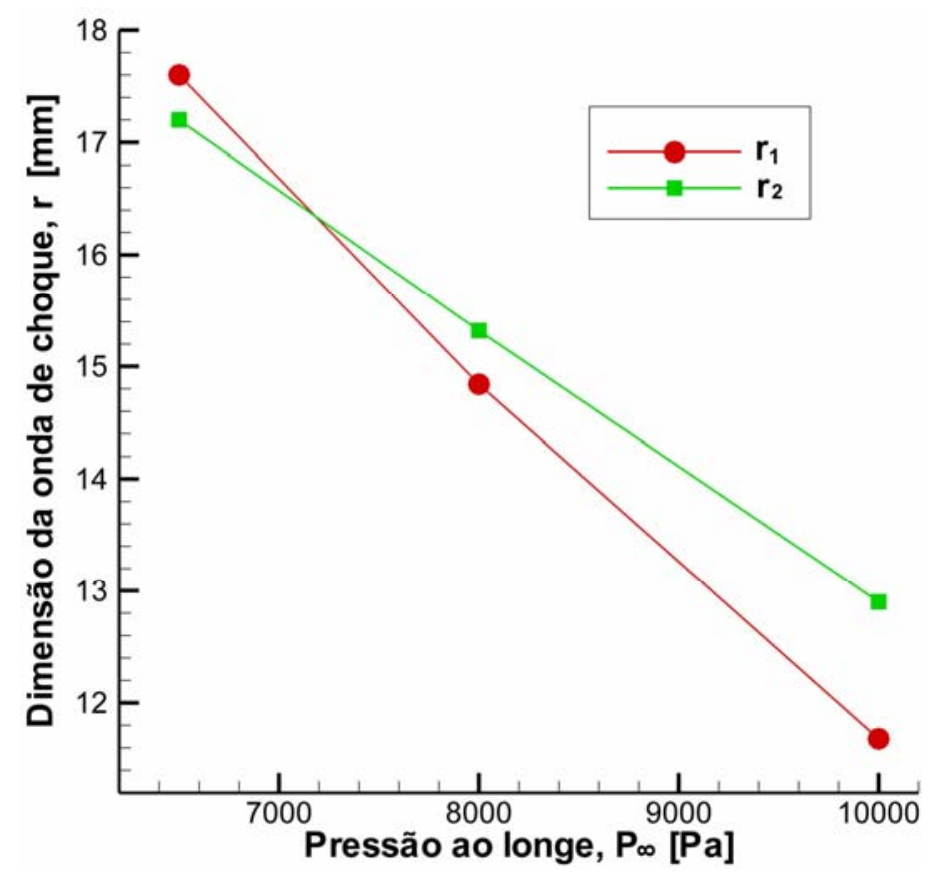

Figura 7.16 Variação da pressão ao longe e dimensão da onda de choque. 


\subsubsection{Independência da malha}

Uma das condições necessárias para demonstrar ou mostrar a convergência do método numérico, quando não se conhece a solução analítica, geralmente isso acontece em fenômenos de grande porte, é utilizar a técnica de independência da malha, isto é, conseguida uma malha para o qual o método resolve numericamente o fenômeno nada garante que a solução achada é a solução que esperávamos para aquele conjunto de condições iniciais e de contorno. É aconselhável se refinar a malha até quando comparadas a soluções numéricas de uma malha mais grossa com uma mais fina o erro não oscile desproporcionalmente, mas bem se estabilize a um valor fixo, próximo a zero.

Para nosso caso da expansão bifásica consideramos quatro tipos de malhas do Teste 11 e para pressões ao longe fixa igual a 8000 [Pa], veja os resultados numéricos na Tabela 7.12.

Tabela 7.12 Independência da malha.

\begin{tabular}{c|c|c|c|c|c}
\hline \hline Teste & $\begin{array}{c}\text { Tempo CPU } \\
\text { [hh:mm:ss:ms] }\end{array}$ & $P_{\infty}[\mathrm{Pa}]$ & Tipo de Malha & $r_{1}[\mathrm{~mm}]$ & $r_{2}[\mathrm{~mm}]$ \\
\hline 11 & $02: 46: 43: 562$ & 8000 & $\mathrm{~A}$ & 14.23 & 14.84 \\
11 & $03: 37: 14: 484$ & 8000 & $\mathrm{~B}$ & 14.15 & 14.69 \\
11 & $04: 33: 46: 859$ & 8000 & $\mathrm{C}$ & 14.46 & 14.97 \\
11 & $07: 11: 9: 468$ & 8000 & $\mathrm{D}$ & 14.56 & 14.83 \\
\hline
\end{tabular}

Pode-se observar que as dimensões da onda de choque variam muito pouco quando a malha é cada vez mais fina. Para o caso do comprimento radial, $r_{1}$, por exemplo, o comprimento radial médio é: 14.35 [mm]. Em quanto ao tempo CPU, notamos que na última malha $\mathrm{D}$, o tempo é muito maior em relação às outras malhas anteriores, onde o tempo CPU aumenta proporcionalmente.

\subsubsection{Comparação com resultados experimentais}

Foram realizados dois testes o Teste 11 e o Teste 04 cujos resultados da dimensão da onda de choque são mostrados na Tabela 7.13 e na Tabela 7.14, respectivamente. Os resultados experimentais (Vieira, 2005) e numéricos (software ShoWPhasT-2D v2), estudados na Cap. 6, são bem próximos, em quanto que os resultados numéricos do código 
computacional $D C D-2 D$ v1 deferem muito dos outros dois. O código computacional $D C D-2 D$ $v 1$ não conseguiu executar o problema bifásico para pressões ao longe muito baixas menores que $2000[\mathrm{~Pa}]$, porém, se aumentássemos a dimensão da geometria do domínio bifásico conseguiríamos executar pressões ao longe mais baixas, e por conseqüência as dimensões da onda de choque aumentariam. Sem embargo, podemos obter as dimensões da onda de choque bem próximas às obtidas experimentalmente e numericamente (ShoWPhasT-2D v2) com pressões ao longe muito diferentes, veja o Teste 04, na Tabela 7.14.

Tabela 7.13 Comparação dos resultados do TESTE 11.

\begin{tabular}{lcccc}
\hline \multicolumn{1}{c}{ TESTE 11 } & $\begin{array}{c}\text { Tipo de } \\
\text { Malha }\end{array}$ & $P_{\infty}[\mathrm{Pa}]$ & $r_{1}[\mathrm{~mm}]$ & $r_{2}[\mathrm{~mm}]$ \\
\hline Experimental & - & 960 & 5.5 & 6.6 \\
ShoWPhasT-2D v2 & $\mathrm{C}$ & 960 & 4.8 & 6.6 \\
DCD-V2 v1 & $\mathrm{C}$ & 8000 & 14.8 & 15.3 \\
\hline
\end{tabular}

Tabela 7.14 Comparação dos resultados do TESTE 04.

\begin{tabular}{lcccc}
\hline \multicolumn{1}{c}{ TESTE 04 } & $\begin{array}{c}\text { Tipo de } \\
\text { Malha }\end{array}$ & $P_{\infty}[\mathrm{Pa}]$ & $r_{1}[\mathrm{~mm}]$ & $r_{2}[\mathrm{~mm}]$ \\
\hline Experimental & - & 80 & 13.8 & 12.4 \\
ShoWPhasT-2D v2 & $\mathrm{C}$ & 80 & 14.1 & 11.5 \\
DCD-V2 v1 & $\mathrm{C}$ & 4000 & 13.91 & 16.36 \\
\hline
\end{tabular}

Conclui-se, por tanto, que o código computacional $D C D-2 D$ v1 consegue capturar a onda de choque sem nenhuma técnica adicional pós-processamento. Quando os resultados numéricos da geometria da onda de choque são comparados com resultados experimentais (Vieira, 2005) eles diferem muito. Então o código não está resolvendo satisfatoriamente as dimensões da onda de choque. Referente ao formato da onda de choque este código não consegue capturar a onda de choque que reflete da parede, possivelmente seja porque as malhas usadas não apresentaram nenhum tipo de refinamento local. 


\section{CONCLUSÕES E CONTINUIDADE DO TRABALHO}

\subsection{Conclusões}

Para o estudo do fenômeno de evaporação rápida (“flashing”) de jatos de fluidos superaquecidos, numa região 2D, estudados e comentados nos capítulos anteriores podemos apresentar as principais conclusões deste trabalho.

- $\quad$ Obtiveram-se as propriedades termodinâmicas dos estados da Pré-expansão bifásica e do estado Pós-onda de evaporação, como mostra a Tabela 6.1. Assim ficam estabelecidas as condições de contorno para a linha inicial da geometria bifásica.

- Aperfeiçoou-se o trabalho feito por Angelo (2004), elaborando-se a versão 2 do software, ShoWPhasT-2D v2. Estas mudanças correspondem aos seguintes tópicos:

$>\quad$ Qualidade da Malha: Com o refinamento da malha ganhou-se maior precisão no formato da onda de choque, tanto na parede quanto no interior da expansão bifásica, quando comparados com os resultados de Vieira (2005), veja Fig. 6.2.

$>$ Condições de contorno: Através de um tratamento especial para as condições de contorno, obtiveram-se linhas de corrente diferentes das linhas de corrente de Angelo (2004) e mais próximos dos resultados de Vieira (2005), nas proximidades da parede. Consequentemente se identificou uma onda refletiva que se encontra com outra onda gerada no interior do domínio, formando dessa maneira o formato geral da onda de choque, este resultado é esboçado na Fig. 6.5.

$>$ Expansão Bifásica: Com a substituição da equação de Estado de Lee-Kesler por tabelas termodinâmicas interpoladas e com rotinas de cálculo, acelerou-se o processo de avaliação das propriedades termodinâmicas. Consequentemente o código computacional ficou mais rápido na execução dos processos.

- Comparação com resultados experimentais de Vieira (2005) e (VIEIRA; SIMÕESMOREIRA, 2007) e, resultados numéricos de Angelo (2004) e (ANGELO; SIMÕESMOREIRA, 2007), realizados no Laboratório SISEA: 
$>$ Com testes experimentais: Em relação à variação das condições de reservatório vinte testes foram realizados para a obtenção da geometria da onda de choque. Os testes numéricos aproximam-se dos testes experimentais com um erro de $41.10 \%$ para o caso do comprimento radial $r_{1}$, e $30.47 \%$ para o caso do comprimento axial $r_{2}$. Para o caso da malha com refinamento o erro (relativo) diminui muito pouco, conforme Tabela 6.3. Estes resultados aparentemente altos se devem a simplificações feitas na geometria do cone evaporativo quando aproximados por um modelo quase-paraboloidal (em 2D é a Linha inicial). As mudanças geométricas na Linha inicial repercutiram nos valores do vetor velocidade avaliado nesta linha. As formas dos choques estão muito parecidas com os resultados experimentais verificadas através das fotos.

> Com testes numéricos: Foi comparado o tempo CPU entre software ShoWPhasT2D v2 e o software ShoWPhasT2D v1. Resultando que a nova versão 2 é muito mais rápida que a versão 1 , veja Tabela 6.4.

- Implementou-se um novo código computacional o $D C D-2 D$ v1 ("DispersionControlled Dissipative - Two-Dimensional version 1"), o qual soluciona numericamente fenômenos não estacionários. Este código baseia-se no esquema "Dispersion-Controlled Dissipative” (DCD), (JIANG, 1993, 2004) que pertence à classe dos esquemas "nonoscillatory shock-capturing”. Para um modelo de falso-transiente do fenômeno de evaporação rápida de líquidos altamente metaestáveis, o código captura diretamente a onda de choque na geometria bifásica sem nenhuma técnica adicional de pósprocessamento, como podem ser observado, por exemplo, na Fig. 7.10. Porém, ainda não apresenta bons resultados para as dimensões da onda de choque quando comparados com os testes experimentais e os resultados numéricos do código ShoWPhasT2D v2. Mais estudos e pesquisas são necessários para explicar este resultado. Este método permitiu a determinação dos campos de velocidades e termodinâmico através de toda região de escoamento. O campo de título foi também determinado. Futuras medições permitirão comparações antes impossíveis. O método de simulação ShoWPhasT-2D v2 não permitia isto. 


\subsection{Continuidade do Trabalho}

As propostas de continuidade deste trabalho são:

- Melhorar a malha com métodos adaptativos ou refinamentos locais mais eficientes em regiões onde os gradientes são maiores.

- Aperfeiçoar o código computacional $D C D-2 D$ v1, para uma nova versão capaz de resolver com maior eficiência nas proximidades das paredes e reduzir o custo computacional, otimizando rotinas de cálculos que empregam tempos maiores.

- Experimentar outras geometrias e/ou problemas de "shock-capturing" com o código $D C D-2 D v 1$, assim como também outros fluidos realísticos. 


\section{REFERÊNCIAS BIBLIOGRÁFICAS}

ALAMIGIR MD.; LIENHARD, J. H. Correlation of Pressure Undershoot During HotWater Depressurization. Journal of Heat Transfer, ASME, Vol. 103, 1981, pp. 52-55.

AL-HENGARI, S. R.; EL-BOUSIFFI, M. A., EL-MUDIR, W. Performance analysis of a MSF desalination unit. Desalination, Vol. 182, 2005, pp. 73- 85.

ANDERSON, J. D. Computational fluid Dynamics: The basics with applications, McGrawHill, New York, 1995, pp. 547.

ANGELO, E. Análise Numérica da Dinâmica da Expansão de Líquidos Superaquecidos em Evaporação Rápida. 2004. 199 p. Tese (Doutorado) - Escola Politécnica, Universidade de São Paulo, São Paulo, 2004.

ANGELO, E. Estudo numérico de jatos evaporativos. 2000. 148 p. Dissertação (Mestrado) - Escola Politécnica, Universidade de São Paulo, São Paulo, 2000.

ANGELO, E.; SIMÕES-MOREIRA, J. R. Numerical Solution of Highly Expanded Flashing Liquid Jets. Journal of Thermophysics and Heat Transfer, Vol. 21, No. 2, 2007, pp. 379-391.

ATHANS, R. E. The rapid expansion of near-critical retrograde fluid. Ph.D. Thesis, Rensselaer Polytechnic Institute, Troy, NY, 1995.

BOLLE, L.; DOWNAR-ZAPOLSKI, P.; FRANCO J.; SEYNHAEVE, J. M. Experimental and theoretical analysis of flashing water flow through a safety valve. Journal of Hazardous Materials, Vol. 46, 1996, pp. 105- 116.

CARAMANA, E. J.; SHASHKOV, M. J.; WHALEN, P. P. Formulations of Artificial Viscosity for Multi-dimensional Shock Wave Computations. Journal of Computational Physics, Vol 144, 1998, pp. 70-97. 
FIGUEIREDO, NATALIE JIMENEZ VERDI DE; SCARAZZATO, TATIANA; ANGELO, E. Determinação das propriedades termodinâmicas através de interpolações e extrapolações a partir de uma tabela para a região de saturação líquido-vapor. In: VII Congresso Brasileiro de Engenharia Química em Iniciação Científica, 2007.

HILL, L. G. An experimental study of evaporation waves in superheated liquids. Ph.D. Thesis, California Institute of Technology, California, 1991.

JIANG, Z. On dispersion-controlled principles for non-oscillatory shock-capturing schemes. Acta Mechanica Sinica, Vol. 20, No. 1, 2004, pp. 1-15.

JIANG, Z. Study on the Finite Difference Theory and Numerical Methods of Weak Solution Problems. Ph.D Thesis, Peking University, Beijing, China, 1993.

JIANG Z.; TAKAYAMA, K.; CHEN, YS. Dispersion conditions for non-oscillatory shock capturing schemes and its applications. Computational Fluid Dynamics J., Vol. 2, 1995, pp. 137-150.

KAHRAMAN, N.; CENGEL, Y. A. Exergy analysis of a MSF distillation plant. Energy Conversion \& Management, Vol. 46, 2005, pp. 2625-2636.

KURSCHAT, T.; CHAVES, H.; MEIER, G. E. A. Complete Adiabatic Evaporation of Highly Superheated Liquid Jets. Journal of Fluid Mechanics, Vol. 236, 1992, pp. 43-59.

LINS N. A. Funções de uma variável complexa. $2^{\text {a }}$ Ed., IMPA, 1996, pp. 468.

MACCORMACK, R. W. The Effect of Viscosity in Hypervelocity Impact Cratering. AIAA Paper, 1969, pp. 69-354.

MACPHEE, A. G., et al. X-ray Imaging of Shock Waves Generated by High-Pressure Fuel Sprays. Science, Vol. 295, No. 1261, 2002. 
OGLE, R. A.; MEGERLE, M. V.; MORRISON, D. R.; CARPENTER, A. R. Explosion Caused by Flashing Liquid in a Process Vessel. Journal of Hazardous Materials, Vol. 115, 2004, pp. 133-140.

PEYRET, R.; TAYLOR, T.D. Computational Methods for Fluid Flow. Springer-Verlag New York Inc, 1983, pp. 358.

PHINASI, G. A.; ULlMANN, A.; DAYAN, A. Modeling of Flashing Two-Phase Flow. Review in Chemical Engineering, Vol. 21, No. (3-4), 2005, pp. 133-164.

PRUGH, R. W. Quantify BLEVE Hazards. Chemical Engineering Progress, Vol. 87, No. 2, 1991, pp. 66-72.

REID, R.C.; PRAUSNITZ, J.M.; POLING, B.E. The properties of gases \& liquids. 4.ed. New York: McGraw-Hill, 1987. 714p.

ROCHETTE, D.; CLAIN, S.; BUSSIÈRE, W.; GENTILS, F. Two-dimensional modelling of internal arc effects in an enclosed MV cell provided with a protection porous filter. Journal of Physics D: Applied Physics, Vol. 40, 2007, pp. 3137- 3144.

SIMÕES-MOREIRA, J. R. Adiabatic evaporation waves. Ph.D. Thesis, Rensselaer Polytechnic Institute, Troy, NY, 1994.

SIMÕES-MOREIRA, J. R. Aplicação da teoria das ondas de evaporação aos jatos evaporativos. 145 p. Tese (Livre Docência) - Escola Politécnica, Universidade de São Paulo, São Paulo, 1999.

SIMÕES-MOREIRA, J. R. Comportamento Termodinâmico de substâncias de complexidade molecular elevada. In: Congresso Ibero-Americano de Engenharia Mecânica, 3, Havana, Cuba, 23 a 26 de setembro, 1997.

SIMÕES-MOREIRA, J. R. Oblique Evaporation Waves. Shock Waves, Vol. 10, 2000, pp. 229-234. 
SIMÕES-MOREIRA, J. R.; BULLARD, C. Pressure drop and flashing mechanisms in expansion devices. International Journal of Refrigeration, Vol. 26, 2003, pp. 840- 848.

SIMÕES-MOREIRA, J. R.; MCCAHAN, S. E.; SHEPHERD, J. E. Complete evaporation waves. In: ASME Fluid Engineering Conference, paper \# 93-FE-7, Washington DC, EUA, June 20-24, 1993.

SIMÕES-MOREIRA, J. R.; SHEPHERD, J. Evaporation waves in superheated dodecane. Journal of Fluid Mechanics, Vol. 382, 1999, pp. 63-68.

STEGER, J. F.; WARMING, R. F. Flux vector splitting of the inviscid gasdynamic equations with applications to finite difference methods. Journal of Computational Physics, Vol. 40, 1981, pp. 263-293.

TENG, H.; JIANG, Z. HAN, Z.; HOSSEINI S. H. R.; TAKAYAMA, K. Numerical investigation of toroidal shock wave focusing in a cylindrical chamber. Shock Wave, Vol. 14, No. 4, 2005, pp. 299-305.

TENG, H.; WANG, C.; DENG, B.; JIANG, Z. Ignition Characteristics of the Shock Wave focusing in Combustive Gases. Chinese Journal of Theoretical and Applied Mechanics, Vol. 39, No. 2, 2007, pp. 171-180.

VIEIRA, M. M. Estudo experimental de jatos evaporativos. 1999. 228 p. Dissertação (Mestrado) - Escola Politécnica, Universidade de São Paulo, São Paulo, 1999.

VIEIRA, M. M. Estudo Experimental da Evaporação de Jatos de Iso-octano Superaquecido. 2005. 372 p. Tese (Doutorado) - Escola Politécnica, Universidade de São Paulo, São Paulo, 2005.

VIEIRA M. M.; SIMÕES-MOREIRA, J. R. Low Pressure Flashing Mechanisms in IsoOctane Liquid Jets. Journal of Fluid Mechanics, Vol. 572, 2007, pp. 121-144.

ZONGMIN H.; JIANG, Z. Wave dynamic processes in cellular detonation reflection from wedges. Acta Mechanica Sinica,Vol. 23, 2007, pp. 33-41. 


\section{APÊNDICE A - ALGORITMOS EM PSEUDOCÓDIGO}

Neste apêndice descreveremos os algoritmos em pseudocódigo para obter as propriedades termodinâmicas do estado metaestável, pós-onda de evaporação e finalmente o algoritmo para o código computacional $D C D-2 D v 1$.

\section{A.1 Estado Metaestável}

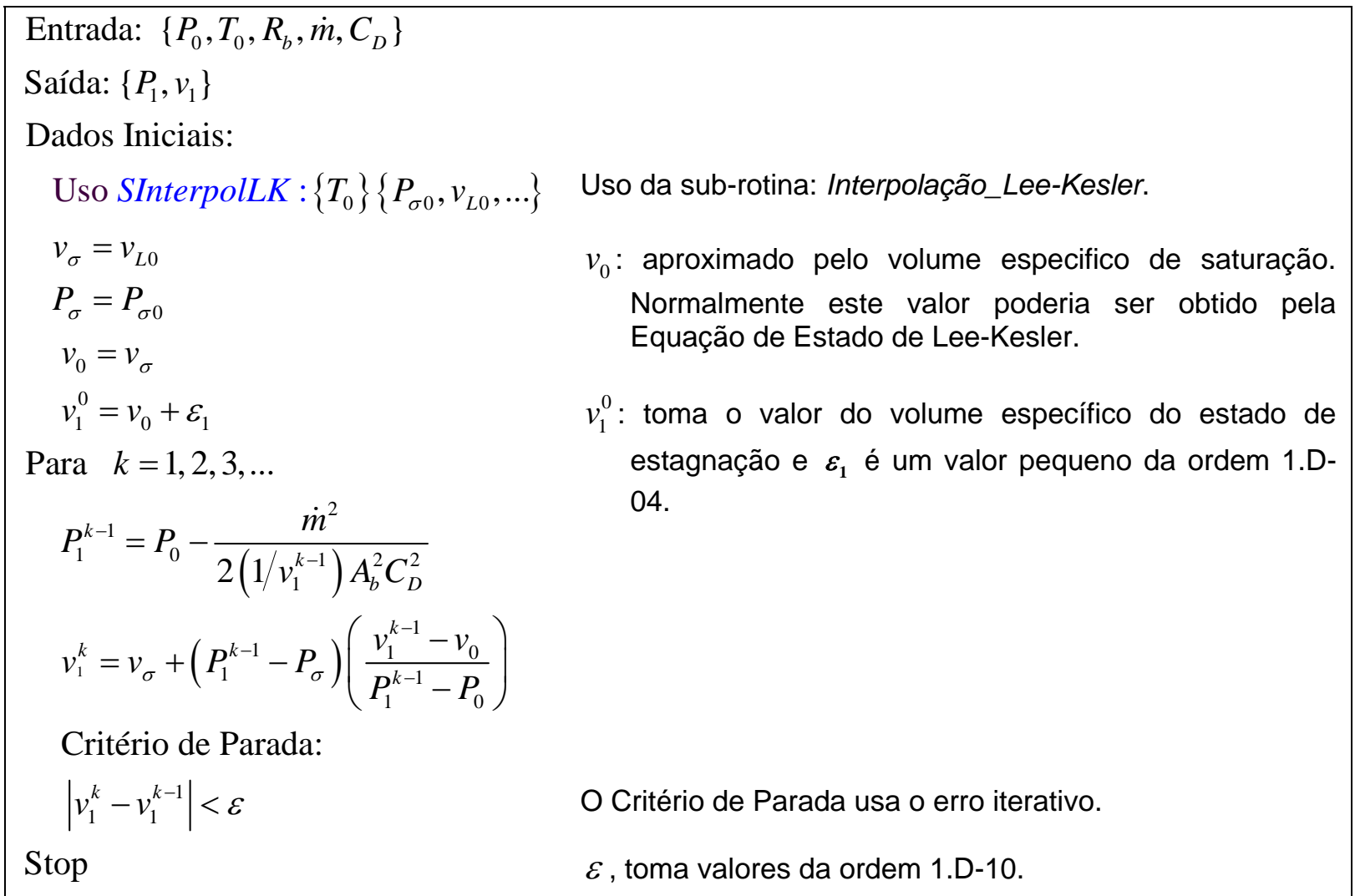

Figura A.1 Algoritmo em pseudocódigo para obter o estado metaestável.

\section{A.2 Propriedades à Jusante da Onda de Evaporação}


Entrada: $\left\{T_{0}, P_{1}, v_{1}\right\}$

Saída: $\left\{T_{2}, P_{2}, x_{2}, v_{2}, h_{2}\right\}$

Dados Iniciais:

$T_{1}=T_{0}$

Uso SInterpolLK : $\left\{T_{1}\right\}\left\{h_{L 1}, \ldots\right\}$

$h_{1}=h_{L 1}$

A temperatura metaestável é aproximada pela temperatura de injeção

Uso da sub-rotina: Interpolação_LeeKesler.

Uso SInterpolLK $:\left\{P_{1}\right\}\left\{T_{\sigma 1}, \ldots\right\}$

$T_{2}^{0}=T_{\sigma 1}$

Uso SInterpolLK: $\left\{T_{2}^{0}\right\}\left\{P_{\sigma 2}^{0}, v_{L 2}^{0}, v_{V 2}^{0}, h_{L 2}^{0}, h_{V 2}^{0}, \ldots\right\}$

$P_{2}^{0}=P_{\sigma 2}^{0}$

$x_{2}^{0}=\frac{2\left(h_{1}-h_{L 2}^{0}\right)+\left(P_{2}^{0}-P_{1}\right)\left(v_{1}+v_{L 2}^{0}\right)}{2\left(h_{V 2}^{0}-h_{L 2}^{0}\right)-\left(P_{2}^{0}-P_{1}\right)\left(v_{V 2}^{0}+v_{L 2}^{0}\right)}$

$v_{2}^{0}=\left(1-x_{2}^{0}\right) v_{L 2}^{0}+x_{2}^{0} v_{V 2}^{0}$

Para $k=1,2,3, \ldots$

$J^{2}=-\left(\frac{P_{2}^{k-1}-P_{1}}{v_{2}^{k-1}-v_{1}}\right)$

Defina-se:

$$
F\left(T_{2}^{k}\right)=\left[\frac{d v_{2}}{d T_{2}}\right]^{k-1}+\frac{1}{J^{2}}\left[\frac{d P_{2}}{d T_{2}}\right]^{k-1}
$$

Critério de parada:

As derivadas entre colchetes são calculadas por qualquer método de diferencias finitas.

$$
\left|T_{2}^{k}-T_{2}^{k-1}\right|<\varepsilon
$$

Senão

$\varepsilon:$ é um número muito pequeno da ordem 1.D-10

Uso SInterpolLK: $\left\{T_{2}^{k}\right\}\left\{P_{\sigma 2}^{k}, v_{L 2}^{k}, v_{V 2}^{k}, h_{L 2}^{k}, h_{V 2}^{k}, \ldots\right\}$

$$
\begin{aligned}
P_{2}^{k} & =P_{\sigma 2}^{k} \\
x_{2}^{k} & =\frac{2\left(h_{1}-h_{L 2}^{k}\right)+\left(P_{2}^{k}-P_{1}\right)\left(v_{1}+v_{L 2}^{k}\right)}{2\left(h_{V 2}^{k}-h_{L 2}^{k}\right)-\left(P_{2}^{k}-P_{1}\right)\left(v_{V 2}^{k}+v_{L 2}^{k}\right)} \\
v_{2}^{k} & =\left(1-x_{2}^{k}\right) v_{L 2}^{k}+x_{2}^{k} v_{V 2}^{k} \\
h_{2}^{k} & =\left(1-x_{2}^{k}\right) h_{L 2}^{k}+x_{2}^{k} h_{V 2}^{k}
\end{aligned}
$$

Stop

$T_{2}=T_{2}^{k}, P_{2}=P_{2}^{k}, \quad x_{2}=x_{2}^{k}, \quad v_{2}=v_{2}^{k}, \quad h_{2}=h_{2}^{k}$

Figura A.2 Algoritmo em pseudocódigo para achar as propriedades à jusante da onda de evaporação. 


\section{A.3 Código DCD-2D v1}

O código computacional $D C D-2 D v 1$ foi feito na linguagem Fortran e está composto de funções e sub-rotinas desenvolvidas pelo autor deste trabalho.

\section{A.3.1 Algumas notações}

i-fluid : Índice do fluido. Indica que classe de fluido é, se:

i-fluid $=1:$ Ar

i-fluid $=2:$ Iso-octano

i_completo: Índice para ativar se a fronteira é completa ou incompleta. Caso seja completa nos estaremos referindo às condições de Dirichlet.

i_completo $=0$ : Incompleto

i_completo $=1:$ Completo

i_simetria: Índice de simetria. Indica se o problema é ou não é simétrico.

i_simetria $=0$ : Não-Simétrico

i_simetria $=1$ : Simétrico

p_posicao: Parâmetro de posição. Indica o fator que multiplicará ao número de nós da Linha inicial da malha, $0 \leq p_{-}$posicao $\leq 1$, o total do produto será o nó escolhido.

i_g: Identificador de geometrias
(a) Para ondas de reflexão:
i_g $=1$
(b) Para um bocal convergente-divergente:
i_g $=2$
(c) Para um bocal supersônico:
i_g $=3$
(d) Para a expansão bifásica de um jato evaporativo: i_g $=4$

\section{A.3.2 Definição de algoritmos em pseudocódigo}

\section{$>\quad$ Algoritmo: Derivada_Dp_Drho}


Entrada: $\left\{T_{1}, p_{1}, x_{1}\right\}$

Saída: $\{\partial p / \partial \rho\}$

1. Uso: SInterpolLK : $\left\{T_{1}\right\}\left\{P_{1 s a t}, v l_{1 s a t}, v v_{1 s a t}, h l_{1 s a t}, h v_{1 s a t}, s l_{1 s a t}, S v_{1 s a t}\right\}$

2. Definição:

$s_{1}=\left(1-x_{1}\right) s l_{1 \text { sat }}+x_{1} s v_{1 s a t}$

$v_{1}=\left(1-x_{1}\right) v l_{1 s a t}+x_{1} v v_{1 s a t}$

$p_{2}=1.05 p_{1}$

$p_{3}=0.95 p_{1}$

3. Uso: SInterpolLK: $\left\{p_{2}\right\}\left\{T_{2 s a t}, v l_{2 s a t}, v v_{2 s a t}, h l_{2 s a t}, h v_{2 s a t}, s l_{2 s a t}, s v_{2 s a t}\right\}$

4. Uso: SInterpolLK: $\left\{p_{3}\right\}\left\{T_{3 s a t}, v l_{3 s a t}, v v_{3 s a t}, h l_{3 s a t}, h v_{3 s a t}, s l_{3 s a t}, s v_{3 s a t}\right\}$

5. Condição isentrópica: $s_{2}=s_{1} ; \quad s_{3}=s_{1}$

6. Definição:

$X_{2}=\left(s_{2}-s l_{2 s a t}\right) /\left(s v_{2 s a t}-s l_{2 s a t}\right)$

$X_{3}=\left(s_{3}-s l_{3 s a t}\right) /\left(s v_{3 s a t}-s l_{3 s a t}\right)$

$\rho_{1}=1 / v_{1} ; \quad \rho_{2}=1 / v_{2} ; \quad \rho_{3}=1 / v_{3} ;$

7. Computar: $\partial p / \partial \rho=\left(p_{2}-p_{3}\right) /\left(\rho_{2}-\rho_{3}\right)$

Figura A.3 Algoritmo em pseudocódigo da Derivada_Dp_Drho.

\section{A.3.3 Definição de sub-rotinas e funções}

Na Tabela A.1 apresentaremos as sub-rotinas e funções mais importante do código $D C D-2 D$ v1. Para simplificar notações cada vez que apareça o sobrescrito "l"significará que ele percorre de 1 a 4, ou seja, $l=\overline{1,4}$. Também, ao inicio década notação se a letra for $F$ então significará que nos estamos referindo a uma função e se a letra for $S$ trata-se de uma subrotina. 
Tabela A.1 Sub-rotinas e funções.

\begin{tabular}{|c|c|c|}
\hline Nome da sub-rotina & Notação & Descrição \\
\hline Velocidade_do_som & FVelocidadeS $:\{T, P, x\}\{c\}$ & \\
\hline Derivada_Dp_Drho & FDerivDpDrho: $\left\{T_{1}, p_{1}, x_{1}\right\}\left\{\frac{\partial p}{\partial \rho}\right\}$ & $\begin{array}{l}\text { Derivada de } p \\
\text { com relação a } \rho\end{array}$ \\
\hline Derivada_Dp_De & FDerivDpDe: $\left\{T_{1}, p_{1}, x_{1}\right\}\left\{\frac{\partial p}{\partial e}\right\}$ & $\begin{array}{l}\text { Derivada de } p \\
\text { com relação a } e\end{array}$ \\
\hline Condição CFL & SCondicaoCFL $:\left\{k, u_{-1}, v_{-2}, c\right\}\{d t$, beta, $t e\}$ & $\begin{array}{l}d t \text { : é o tamanho de } \\
\text { passo do tempo }\end{array}$ \\
\hline Corpo & SCorpo: $\left\{U_{0 l}, U_{0 l_{-}}, \rho_{1}, T_{1}, p_{1}, e_{1}\right.$, beta, $\left.k\right\}\left\{F_{l}\right\}$ & \\
\hline $\begin{array}{l}\text { Cálculo das variáveis } \\
\text { primitivas }\end{array}$ & SCalculoVP: $\left\{M, N, k, U_{l}, T_{10}\right\}\left\{\rho_{1}, u_{-}, v_{-1}, p_{1}, e_{1}, T_{1}, x t_{1}, A M a c_{1}, c_{1}\right\}$ & \\
\hline Boundary Condition & SBoundaryC $:\left\{k, U_{01}, \rho_{1}, u_{-1}, v_{-1}, p_{1}, e_{1}, T_{1}, x t_{1}, A M a c_{1}\right\}\left\{U_{l}\right\}$ & \\
\hline Cond_Contorno_Chapéu & SCondicaoCC: $\left\{U_{l}\right\}\left\{U_{I_{-}}\right\}$ & \\
\hline NormaDeUmaMatriz & SNormaM $:\left\{U_{011}, N, M\right\}\left\{E U_{l}\right\}$ & \\
\hline ErroRelativo & $\begin{aligned} \text { SErroR: } & :\left\{\text { is, it } t_{0}, i t, k, l c, t e, d t, \text { gam, Erro, } \rho_{i}, p_{i}, T_{i}, \text { AMac }_{i}, T_{10}\right\} \\
& \left\{\rho_{1}, u_{-1}, v_{-}, p_{1}, e_{1}, T_{1}, x t_{1}, A M a c_{1}\right\}\end{aligned}$ & \\
\hline $\begin{array}{l}\text { Cálculo_do_Jacobiano_ } \\
\text { e_Métricas }\end{array}$ & SCalculoJM $:\{M, N, x, y, d x, d y\}\left\{A J, \xi_{x}, \xi_{y}, \eta_{x}, \eta_{y}\right\}$ & \\
\hline
\end{tabular}

\section{A.3.4 Algoritmo}

\section{DADOS DE ENTRADA}

i_fluido, i_completo, i_simetria, it, $i \_g$,

$x, y, \rho_{0}, u_{0}, v_{0}, p_{0}, e_{0}, T_{0}, x t_{0}$, AMac $_{0}$.

Uso SCalculoJM : $\{M, N, x, y, d x, d y\}\left\{A J, \xi_{x}, \xi_{y}, \eta_{x}, \eta_{y}\right\}$

$A J$ : Jacobiano;

CONDIÇÃO INICIAL

$M, N$ :dimensão da malha.

$V_{0}^{2}(i, j)=u_{0}^{2}(i, j)+v_{0}^{2}(i, j)$

$U_{1}(i, j)=\rho_{0}(i, j)$

$U_{2}(i, j)=\rho_{0}(i, j) u_{0}(i, j), \quad i=\overline{0, N+1}, j=\overline{0, M+1}$

$U_{3}(i, j)=\rho_{0}(i, j) v_{0}(i, j)$

$U_{4}(i, j)=\rho_{0}(i, j)\left[e_{0}(i, j)+0.5 V_{0}^{2}(i, j)\right]$

$U_{0 l}(i, j)=U_{l}(i, j), i=\overline{0, N+1}, j=\overline{0, M+1}$

$U_{0 l_{-}}(i, j)=U_{l}(i, j) / A J(i, j), i=\overline{0, N+1}, j=\overline{0, M+1}$ 


\section{MÉTODO DE RUNGE KUTTA DE ORDEM 2}

TOL=1.E-008; $\mathrm{ERRO}=1 . \mathrm{E}+008 ; \mathrm{k}=1 ; \mathrm{l}_{\mathrm{C}}=1 ; \mathrm{it} 0=0 ; \quad$ te=0.D0

TOL: Tolerância

Fazer enquanto: $\mathrm{k} \leq$ it $\wedge$ ERRO $\geq \mathrm{TOL}$

Uso SCondicaoCFL: $\left\{k, u_{-1}, v_{-2}, c\right\}\{d t$, beta,$t e\}$

\section{PARTE PREDITOR}

Uso SCorpo: $\left\{U_{0 l}, U_{0 l_{-}}, \rho_{1}, T_{1}, p_{1}, e_{1}\right.$, beta, $\left.k\right\}\left\{F_{l}\right\}$

$U_{l_{-}}(i, j)=U_{0 l_{-}}(i, j)+d t F_{l}(i, j), i=\overline{1, N}, j=\overline{1, M}$

$U_{l}(i, j)=A J(i, j) U_{l}(i, j), i=\overline{1, N}, j=\overline{1, M}$

Uso SCalculoVP: $\left\{M, N, k, U_{1}, T_{10}\right\}\left\{\rho_{1}, u_{-1}, v_{-1}, p_{1}, e_{1}, T_{1}, x t_{1}, A M a c_{1}, c_{1}\right\}$

Uso SBoundaryC: $\left\{k, U_{0 l}, \rho_{1}, u_{-1}, v_{-1}, p_{1}, e_{1}, T_{1}, x t_{1}\right.$, AMac $\left._{1}\right\}\left\{U_{l}\right\}$

Uso SCondicaoCC: $\left\{U_{l}\right\}\left\{U_{l_{-}}\right\}$

\section{PARTE CORRETOR}

Uso SCorpo: $\left\{U_{l}, U_{l_{-}}, \rho_{1}, T_{1}, p_{1}, e_{1}\right.$, beta,$\left.k\right\}\left\{F_{l a}\right\}$

$U_{l_{-}}(i, j)=U_{0 l_{-}}(i, j)+0.5 d t\left[F_{l}(i, j)+F_{l a}(i, j)\right], i=\overline{1, N}, j=\overline{1, M}$

$U_{l}(i, j)=A J(i, j) U_{l_{-}}(i, j), i=\overline{1, N}, j=\overline{1, M}$

Uso SCalculoVP: $\left\{M, N, k, U_{1}, T_{10}\right\}\left\{\rho_{1}, u_{-1}, v_{-} 1, p_{1}, e_{1}, T_{1}, x t_{1}, A M a c_{1}, c_{1}\right\}$

Uso SBoundaryC : $\left\{k, U_{0 l}, \rho_{1}, u_{-1}, v_{-1}, p_{1}, e_{1}, T_{1}, x t_{1}, A_{M a c_{1}}\right\}\left\{U_{l}\right\}$

Uso SCondicaoCC: $\left\{U_{l}\right\}\left\{U_{l_{-}}\right\}$

ERRO TEMPORAL ITERATIVO

$U_{01 l}(i, j)=U_{l}(i, j)-U_{0 l}(i, j), i=\overline{0, N+1}, j=\overline{0, M+1}$

ERRO, INCLUINDO OS PONTOS DO CONTORNO

Uso SNormaM : $\left\{U_{011}, N, M\right\}\left\{E U_{l}\right\}$

Erro $=\max \left\{E U_{l}\right\}$

Se Erro < TOL então STOP

RECUPERAÇÃO DA SOLUÇÃO

$U_{0 l}(i, j)=U_{l}(i, j), i=\overline{0, N+1}, j=\overline{0, M+1}$

$U_{0 l_{-}}(i, j)=U_{l_{-}}(i, j), i=\overline{0, N+1}, j=\overline{0, M+1}$

ERRO RELATIVO

Uso SErroR: $\left\{\right.$ is, it $_{0}, i t, k, l c, t e, d t$, gam, Erro, $\left.\rho_{i}, p_{i}, T_{i}, A M a c_{i}, T_{10}\right\}$

$$
\left\{\rho_{1}, u_{-1}, v_{-1}, p_{1}, e_{1}, T_{1}, x t_{1}, A M a c_{1}\right\}
$$

$\mathrm{k}=\mathrm{k}+1$

Fim de fazer

Figura A.4 Algoritmo em pseudocódigo do código computacional $D C D-2 D$ v1. 


\section{APÊNDICE B - CONDIÇÕES DE CONTORNO}

Para o caso do fenômeno de evaporação rápida em jatos de líquidos metaestáveis na região bifásica assimétrica 2D calculamos as condições de contorno na Linha inicial, Linha de simetria, Linha ao longe e Linha de parede. As condições de contorno numéricas usadas são em relação à Eqs. (5.39)-(5.40).

Para o cálculo do volume específico do líquido saturado, vl e o volume específico do vapor saturado, $v v$, avaliados no contorno, se usa a sub-rotina: Interpolação_Lee-Kesler, dada na Tabela D.5 do Apêndice D. A velocidade do som avaliada no contorno, $c$, é calculada usando a função: Velocidade_do_som, ver Tabela A.1 do Apêndice A, esta função precisa de três propriedades termodinâmicas de entrada, a saber, $\{T, P, x\}$

\section{B.1 Linha inicial}

Tabela B.1 Valores de contorno na Linha inicial.

\begin{tabular}{l|c|l}
\hline Dados usados & Valor dado & \multicolumn{1}{|c}{ Valor calculado } \\
\hline$P_{I}$ & $P_{2}$ & \\
$X_{I}$ & $x_{2}$ & Eq. (B.1) \\
$\mathrm{u}_{\mathrm{I}}$ & & Eq. (B.1) \\
$\mathrm{V}_{\mathrm{I}}$ & $T_{2}$ & $\rho_{2}=\frac{1}{v_{2}}$ \\
$T_{I}$ & 1,0 & $q_{I}=\sqrt{\mathrm{u}_{\mathrm{I}}^{2}+\mathrm{v}_{\mathrm{I}}^{2}} \Rightarrow c_{I}=\frac{q_{I}}{M_{I}}=q_{I}$ \\
$\rho_{I}$ & & \\
$M_{I}$ & &
\end{tabular}

As propriedades termodinâmicas: $\left\{P_{2}, x_{2}, T_{2}, v_{2}\right\}$ pertence ao estado pós-onda de evaporação e são dados na Tabela 6.1. 


\section{- Cálculo das componentes do vetor velocidade}

Da Fig. 2.6a, podemos obter a seguinte igualdade:

$$
\left|\vec{V}_{2}\right|=\frac{W_{2}}{\operatorname{sen}(\theta+\beta)}
$$

Portanto, definimos as componentes do vetor velocidade da seguinte forma:

$$
\begin{aligned}
& \mathrm{u}_{I}=\left|\vec{V}_{2}\right| \cos (\theta) \\
& \mathrm{v}_{I}=\left|\vec{V}_{2}\right| \operatorname{sen}(\theta)
\end{aligned}
$$

onde $W_{2}$ é a componente normal do vetor velocidade $\vec{V}_{2}, \theta$ e $\beta$ são os ângulos de giro e onda, respectivamente, todos eles são dados na Tabela 6.1.

\begin{tabular}{|c|c|c|}
\hline Dados usados & Valor dado & Valor calculado \\
\hline$P_{S}$ & & Extrapolação de ordem zero \\
\hline$x_{S}$ & & Extrapolação de ordem zero \\
\hline $\mathrm{u}_{\mathrm{s}}$ & & Extrapolação de ordem zero \\
\hline $\mathrm{v}_{\mathrm{S}}$ & 0,0 & \\
\hline$T_{S}$ & & Uso SInterpolLK $:\left\{P_{S}\right\}\left\{T_{S}, v l_{S}, v v_{S}, \ldots\right\}$ \\
\hline$\rho_{S}$ & & $v_{S}=\left(1-x_{S}\right) v l_{S}+x_{S} v v_{S} \Rightarrow \rho_{I}=\frac{1}{v_{I}}$ \\
\hline$c_{S}$ & & Uso FVelocidadeS : $\left\{T_{S}, P_{S}, x_{S}\right\}\left\{c_{S}\right\}$ \\
\hline$M_{S}$ & & $q_{S}=\sqrt{\mathrm{u}_{\mathrm{S}}^{2}+\mathrm{v}_{\mathrm{S}}^{2}} \Rightarrow M_{S}=\frac{q_{S}}{c_{S}}$ \\
\hline
\end{tabular}

\section{B.2 Linha de simetria}

Tabela B.2 Valores de contorno na Linha de simetria. 


\section{B.3 Linha ao longe}

Tabela B.3 Valores de contorno na Linha ao longe.

\begin{tabular}{|c|c|c|}
\hline Dados usados & Valor dado & Valor calculado \\
\hline$P_{\infty}$ & $\checkmark$ & \\
\hline$x_{\infty}$ & & Extrapolação de ordem zero \\
\hline $\mathrm{u}_{\infty}$ & & Extrapolação de ordem zero \\
\hline $\mathrm{v}_{\infty}$ & & Extrapolação de ordem zero \\
\hline$T_{\infty}$ & & Uso SInterpolLK $:\left\{P_{\infty}\right\}\left\{T_{\infty}, v l_{\infty}, v v_{\infty}, \ldots\right\}$ \\
\hline$\rho_{\infty}$ & & $v_{\infty}=\left(1-x_{\infty}\right) v l_{\infty}+x_{\infty} v v_{\infty} \Rightarrow \rho_{\infty}=\frac{1}{v_{\infty}}$ \\
\hline$C_{\infty}$ & & Uso FVelocidadeS : $\left\{T_{\infty}, P_{\infty}, x_{\infty}\right\}\left\{c_{\infty}\right\}$ \\
\hline$M_{\infty}$ & & $q_{\infty}=\sqrt{\mathrm{u}_{\infty}^{2}+\mathrm{v}_{\infty}^{2}} \Rightarrow M_{\infty}=\frac{q_{\infty}}{C_{\infty}}$ \\
\hline
\end{tabular}

\section{B.4 Linha de parede}

Tabela B.4 Valores de contorno na Linha de parede.

\begin{tabular}{|c|c|c|}
\hline Dados usados & Valor dado & Valor calculado \\
\hline$P_{W}$ & & Extrapolação de ordem zero \\
\hline$x_{W}$ & & Extrapolação de ordem zero \\
\hline $\mathrm{u}_{\mathrm{W}}$ & 0,0 & \\
\hline $\mathrm{v}_{\mathrm{W}}$ & & Extrapolação de ordem zero \\
\hline$T_{W}$ & & Uso SInterpolLK $:\left\{P_{W}\right\}\left\{T_{W}, v l_{W}, v v_{W}, \ldots\right\}$ \\
\hline$\rho_{W}$ & & $v_{W}=\left(1-x_{W}\right) v l_{W}+x_{W} v v_{W} \Rightarrow \rho_{W}=\frac{1}{v_{W}}$ \\
\hline$c_{W}$ & & Uso FVelocidadeS : $\left\{T_{w}, P_{W}, x_{w}\right\}\left\{c_{w}\right\}$ \\
\hline$M_{W}$ & & $q_{W}=\sqrt{\mathrm{u}_{\mathrm{W}}^{2}+\mathrm{v}_{\mathrm{W}}^{2}} \Rightarrow M_{W}=\frac{q_{W}}{c_{W}}$ \\
\hline
\end{tabular}




\section{APÊNDICE C - CÁLCULO DA MATRIZ JACOBIANA}

Neste apêndice descreveremos os cálculos envolvidos na obtenção das matrizes jacobianas $A$ e $B$, definidas por:

$$
\begin{aligned}
& A=\frac{\partial F}{\partial U} \\
& B=\frac{\partial G}{\partial U}
\end{aligned}
$$

onde $U$ é a variável de estado (ou variável conservativa) , $F$ e $G$ são as funções de fluxo, definidas na Eq. (5.2):

$$
\begin{aligned}
& F=A U \\
& G=B U
\end{aligned}
$$

A equação de estado usada nestes cálculos corresponde à Equação de estado de LeeKesler. De Eq. (C.1), temos:

$$
A=\left(\begin{array}{llll}
\frac{\partial F_{1}}{\partial U_{1}} & \frac{\partial F_{1}}{\partial U_{2}} & \frac{\partial F_{1}}{\partial U_{3}} & \frac{\partial F_{1}}{\partial U_{4}} \\
\frac{\partial F_{2}}{\partial U_{1}} & \frac{\partial F_{2}}{\partial U_{2}} & \frac{\partial F_{2}}{\partial U_{3}} & \frac{\partial F_{2}}{\partial U_{4}} \\
\frac{\partial F_{3}}{\partial U_{1}} & \frac{\partial F_{3}}{\partial U_{2}} & \frac{\partial F_{3}}{\partial U_{3}} & \frac{\partial F_{3}}{\partial U_{4}} \\
\frac{\partial F_{4}}{\partial U_{1}} & \frac{\partial F_{4}}{\partial U_{2}} & \frac{\partial F_{4}}{\partial U_{3}} & \frac{\partial F_{4}}{\partial U_{4}}
\end{array}\right) \quad B=\left(\begin{array}{lllll}
\frac{\partial G_{1}}{\partial U_{1}} & \frac{\partial G_{1}}{\partial U_{2}} & \frac{\partial G_{1}}{\partial U_{3}} & \frac{\partial G_{1}}{\partial U_{4}} \\
\frac{\partial G_{2}}{\partial U_{1}} & \frac{\partial G_{2}}{\partial U_{2}} & \frac{\partial G_{2}}{\partial U_{3}} & \frac{\partial G_{2}}{\partial U_{4}} \\
\frac{\partial G_{3}}{\partial U_{1}} & \frac{\partial G_{3}}{\partial U_{2}} & \frac{\partial G_{3}}{\partial U_{3}} & \frac{\partial G_{3}}{\partial U_{4}} \\
\frac{\partial G_{4}}{\partial U_{1}} & \frac{\partial G_{4}}{\partial U_{2}} & \frac{\partial G_{4}}{\partial U_{3}} & \frac{\partial G_{4}}{\partial U_{4}}
\end{array}\right)
$$

A variável de estado $U$ e as funções fluxo $F$ e $G$ são definidas por:

$$
U=\left[\begin{array}{c}
\rho \\
\rho \mathrm{u} \\
\rho \mathrm{v} \\
E
\end{array}\right] \quad F=\left[\begin{array}{c}
\rho \mathrm{u} \\
\rho \mathrm{u}^{2}+p \\
\rho \mathrm{uv} \\
(E+p) \mathrm{u}
\end{array}\right] \quad G=\left[\begin{array}{c}
\rho \mathrm{v} \\
\rho \mathrm{uv} \\
\rho \mathrm{v}^{2}+p \\
(E+p) \mathrm{v}
\end{array}\right]
$$


respectivamente. Onde as variáveis primitivas são: $\{\rho, \mathrm{u}, \mathrm{v}, p, e\}$, com a energia interna específica, $e$ satisfazendo a seguinte equação:

$$
E=\rho e+\frac{1}{2} \rho\left(\mathrm{u}^{2}+\mathrm{v}^{2}\right)
$$

e, $E$ a energia interna total. Assim, as componentes da variável de estado e das funções fluxo são expressas em função das variáveis primitivas, dadas por:

$$
\begin{aligned}
& U_{1}=\rho \quad F_{1}=\rho \mathrm{u} \quad G_{1}=\rho \mathrm{v} \\
& U_{2}=\rho \mathrm{u} \quad F_{2}=\rho \mathrm{u}^{2}+p \quad G_{2}=\rho \mathrm{uv} \\
& U_{3}=\rho \mathrm{v} \quad F_{3}=\rho \mathrm{uv} \quad G_{3}=\rho \mathrm{v}^{2}+p \\
& U_{4}=E \quad F_{4}=(E+p) \mathrm{u} \quad G_{4}=(E+p) \mathrm{v}
\end{aligned}
$$

onde as componentes da variável de estado: $\left\{U_{1}, U_{2}, U_{3}, U_{4}\right\}$ são chamadas de componentes conservativas. As variáveis primitivas (a exceção da pressão $p$ ) por sua vez podem ser expressas em função das componentes conservativas do seguinte modo:

$$
\rho=U_{1}, \quad \mathrm{u}=\frac{U_{2}}{U_{1}}, \quad \mathrm{v}=\frac{U_{3}}{U_{1}}, \quad e=\frac{U_{4}}{U_{1}}-\frac{1}{2}\left(\frac{U_{2}^{2}+U_{3}^{2}}{U_{1}^{2}}\right), \quad p(\rho, e)
$$

Substituindo Eq. (C.7) em Eq. (C.6), temos as componentes das funções fluxos dadas por:

$$
\begin{array}{lll}
F_{1}=U_{2} & G_{1}=U_{3} \\
F_{2}=\frac{U_{2}^{2}}{U_{1}}+p(\rho, e) & G_{2}=\frac{U_{2} U_{3}}{U_{1}} \\
F_{3}=\frac{U_{2} U_{3}}{U_{1}} & G_{3}=\frac{U_{3}^{2}}{U_{1}}+p(\rho, e) \\
F_{4}=\frac{U_{2} U_{4}}{U_{1}}+p(\rho, e) \frac{U_{2}}{U_{1}} & G_{4}=\frac{U_{3} U_{4}}{U_{1}}+p(\rho, e) \frac{U_{3}}{U_{1}}
\end{array}
$$

A pressão $p=p(\rho, e)$ é uma função que depende da densidade e da energia interna específica e não se tem uma fórmula explícita para seu cálculo. Na expansão bifásica a 
pressão e a temperatura são dependentes, pelo que se acostuma tomar o par de propriedades termodinâmicas independentes, por exemplo: $\{T, x\}$ ou $\{P, x\}$, onde $x$ é o título.

Dados $F$ e $G$ em Eq. (C.8) podemos calcular suas derivadas em relação das componentes conservativas. Assim,

Matriz jacobiana $A$ :

$$
\begin{array}{llll}
\frac{\partial F_{1}}{\partial U_{1}}=0 & \frac{\partial F_{1}}{\partial U_{2}}=1 & \frac{\partial F_{1}}{\partial U_{3}}=0 & \frac{\partial F_{1}}{\partial U_{4}}=0 \\
\frac{\partial F_{2}}{\partial U_{1}}=? & \frac{\partial F_{2}}{\partial U_{2}}=? & \frac{\partial F_{2}}{\partial U_{3}}=? & \frac{\partial F_{2}}{\partial U_{4}}=? \\
\frac{\partial F_{3}}{\partial U_{1}}=-\frac{U_{2} U_{3}}{U_{1}^{2}} & \frac{\partial F_{3}}{\partial U_{2}}=\frac{U_{3}}{U_{1}} & \frac{\partial F_{3}}{\partial U_{3}}=\frac{U_{2}}{U_{1}} & \frac{\partial F_{3}}{\partial U_{4}}=0 \\
\frac{\partial F_{4}}{\partial U_{1}}=? & \frac{\partial F_{4}}{\partial U_{2}}=? & \frac{\partial F_{4}}{\partial U_{3}}=? & \frac{\partial F_{4}}{\partial U_{4}}=?
\end{array}
$$

Matriz jacobiana $B$ :

$$
\begin{array}{llll}
\frac{\partial G_{1}}{\partial U_{1}}=0 & \frac{\partial G_{1}}{\partial U_{2}}=0 & \frac{\partial G_{1}}{\partial U_{3}}=1 & \frac{\partial G_{1}}{\partial U_{4}}=0 \\
\frac{\partial G_{2}}{\partial U_{1}}=-\frac{U_{2} U_{3}}{U_{1}^{2}} & \frac{\partial G_{2}}{\partial U_{2}}=\frac{U_{3}}{U_{1}} & \frac{\partial G_{2}}{\partial U_{3}}=\frac{U_{2}}{U_{1}} & \frac{\partial G_{2}}{\partial U_{4}}=0 \\
\frac{\partial G_{3}}{\partial U_{1}}=? & \frac{\partial G_{3}}{\partial U_{2}}=? & \frac{\partial G_{3}}{\partial U_{3}}=? & \frac{\partial G_{3}}{\partial U_{4}}=? \\
\frac{\partial G_{4}}{\partial U_{1}}=? & \frac{\partial G_{4}}{\partial U_{2}}=? & \frac{\partial G_{4}}{\partial U_{3}}=? & \frac{\partial G_{4}}{\partial U_{4}}=?
\end{array}
$$

O cálculo das derivadas com sinal de interrogação requer um tratamento especial, mas antes disso calculemos outras derivadas:

$$
\begin{array}{ll}
\frac{\partial \rho}{\partial U_{1}}=1 & \frac{\partial e}{\partial U_{1}}=-\frac{U_{4}}{U_{1}^{2}}+\left(\frac{U_{2}^{2}+U_{3}^{2}}{U_{1}^{3}}\right) \\
\frac{\partial \rho}{\partial U_{2}}=0 & \frac{\partial e}{\partial U_{2}}=-\frac{U_{2}}{U_{1}^{2}} \\
\frac{\partial \rho}{\partial U_{3}}=0 & \frac{\partial e}{\partial U_{3}}=-\frac{U_{3}}{U_{1}^{2}} \\
\frac{\partial \rho}{\partial U_{4}}=0 & \frac{\partial e}{\partial U_{4}}=\frac{1}{U_{1}}
\end{array}
$$


- Cálculo das derivadas de $F_{2}$ :

$$
\begin{aligned}
& F_{2}=F_{2 a}+F_{2 b} \\
& F_{2 a}=\frac{U_{2}^{2}}{U_{1}} \\
& F_{2 b}=p(\rho, e)
\end{aligned}
$$

Logo

$$
\begin{array}{ll}
\frac{\partial F_{2 a}}{\partial U_{1}}=-\frac{U_{2}^{2}}{U_{1}^{2}} & \frac{\partial F_{2 b}}{\partial U_{k}}=\frac{\partial p}{\partial \rho} \frac{\partial \rho}{\partial U_{k}}+\frac{\partial p}{\partial e} \frac{\partial e}{\partial U_{k}}, \quad k=1,4 \\
\frac{\partial F_{2 a}}{\partial U_{2}}=\frac{2 U_{2}}{U_{1}} & \\
\frac{\partial F_{2 a}}{\partial U_{3}}=0 & \\
\frac{\partial F_{2 a}}{\partial U_{4}}=0 &
\end{array}
$$

O cálculo das derivadas $\frac{\partial \rho}{\partial U_{k}}$ e $\frac{\partial e}{\partial U_{k}}$ estão dadas na Eq. (C.11) e para o cálculo das derivadas $\frac{\partial p}{\partial \rho}$ e $\frac{\partial p}{\partial e}$, usamos dois sub-rotinas: Derivada_Dp_Drho e Derivada_Dp_De definidas na Tabela A.1 do Apêndice A. Dessa forma completamos as derivadas de $F_{2}$.

- Cálculo das derivadas de $F_{4}$ :

$$
\begin{aligned}
& F_{4}=F_{4 a}+F_{4 b c} \\
& F_{4 a}=\frac{U_{2} U_{4}}{U_{1}} \\
& F_{4 b c}=F_{4 b} F_{4 c} \\
& F_{4 b}=p(\rho, e)=F_{2 b} \\
& F_{4 c}=\frac{U_{2}}{U_{1}}
\end{aligned}
$$

Logo, 


$$
\begin{array}{llrl}
\frac{\partial F_{4 a}}{\partial U_{1}}=-\frac{U_{2} U_{4}}{U_{1}^{2}} & \frac{\partial F_{4 c}}{\partial U_{1}}=-\frac{U_{2}}{U_{1}^{2}} & \frac{\partial F_{4 b c}}{\partial U_{k}}=F_{4 c} \frac{\partial F_{4 b}}{\partial U_{k}}+F_{4 b} \frac{\partial F_{4 c}}{\partial U_{k}}, \\
\frac{\partial F_{4 a}}{\partial U_{2}}=\frac{U_{4}}{U_{1}} & \frac{\partial F_{4 c}}{\partial U_{2}}=\frac{1}{U_{1}} & k=1,4 \\
\frac{\partial F_{4 a}}{\partial U_{3}}=0 & \frac{\partial F_{4 c}}{\partial U_{3}}=0 & \\
\frac{\partial F_{4 a}}{\partial U_{4}}=\frac{U_{2}}{U_{1}} & \frac{\partial F_{4 c}}{\partial U_{4}}=0
\end{array}
$$

- Cálculo das derivadas de $G_{3}$ :

$$
\begin{aligned}
& G_{3}=G_{3 a}+G_{3 b} \\
& G_{3 a}=\frac{U_{3}^{2}}{U_{1}} \\
& G_{3 b}=p(\rho, e)
\end{aligned}
$$

Logo,

$$
\begin{array}{ll}
\frac{\partial G_{3 a}}{\partial U_{1}}=-\frac{U_{3}^{2}}{U_{1}^{2}} & \frac{\partial G_{3 b}}{\partial U_{k}}=\frac{\partial F_{2 b}}{\partial U_{k}}, \quad k=1,4 \\
\frac{\partial G_{3 a}}{\partial U_{2}} & =0 \\
\frac{\partial G_{3 a}}{\partial U_{3}} & =\frac{2 U_{3}}{U_{1}} \\
\frac{\partial G_{3 a}}{\partial U_{4}} & =0
\end{array}
$$

- Cálculo das derivadas de $G_{4}$ :

$$
\begin{aligned}
G_{4} & =G_{4 a}+G_{4 b c} \\
G_{4 a} & =\frac{U_{3} U_{4}}{U_{1}} \\
G_{4 b c} & =G_{4 b} G_{4 c} \\
G_{4 b} & =p(\rho, e)=G_{3 b} \\
G_{4 c} & =\frac{U_{3}}{U_{1}}
\end{aligned}
$$

Logo, 


$$
\begin{array}{llrlrl}
\frac{\partial G_{4 a}}{\partial U_{1}}=-\frac{U_{3} U_{4}}{U_{1}^{2}} & \frac{\partial G_{4 c}}{\partial U_{1}}=-\frac{U_{3}}{U_{1}^{2}} & \frac{\partial G_{4 b c}}{\partial U_{k}}=G_{4 b} \frac{\partial G_{4 c}}{\partial U_{k}}+G_{4 c} \frac{\partial G_{4 b}}{\partial U_{k}}, \\
\frac{\partial G_{4 a}}{\partial U_{2}}=0 & \frac{\partial G_{4 c}}{\partial U_{2}}=0 & k=1,4 \\
\frac{\partial G_{4 a}}{\partial U_{3}}=\frac{U_{4}}{U_{1}} & \frac{\partial G_{4 c}}{\partial U_{3}}=\frac{1}{U_{1}} & \\
\frac{\partial G_{4 a}}{\partial U_{4}}=\frac{U_{3}}{U_{1}} & \frac{\partial G_{4 c}}{\partial U_{4}}=0 &
\end{array}
$$

Assim temos calculado as componentes das matrizes jacobianas $A$ e $B$. 


\section{APÊNDICE D - EQUAÇÃO DE ESTADO DE LEE-KESLER}

Neste Apêndice obteremos a tabela das propriedades termodinâmicas na curva de saturação, assim como também, a interpolação das mesmas para uma variável termodinâmica específica. Além disso, apresentamos o algoritmo em pseudocódigo para o cálculo da subrotina de Interpolação de Lee-Kesler.

\section{D.1 Cálculo da Tabela Termodinâmica}

Definamos algumas notações:

$$
\begin{array}{ll}
Z_{\text {comp }}: & \text { Fator de compressibilidade de um fluido teste } \\
Z_{\text {comp }}^{(r)} & : \text { Fator de compressibilidade de um fluido de referência } \\
Z_{\text {comp }}^{(0)} & : \text { Fator de compressibilidade de um fluido de simples } \\
Z_{c} & : \text { Fator de compressibilidade crítica } \\
\varpi & : \text { Fator de acentricidade } \\
\varpi^{(r)} \quad: \text { Fator de acentricidade de um fluido de referência } \\
P_{c} \quad: \text { Pressão crítica } \\
T_{c} \quad: \text { Temperatura crítica } \\
T_{r d} \quad: \text { Temperatura reduzida } \\
p_{r d} \quad: \text { Pressão reduzida }
\end{array}
$$

Por definição a temperatura reduzida é dada por:

$$
T_{r d}=\frac{T}{T_{c}}
$$

e a pressão reduzida por:

$$
P_{r d}=\frac{P}{P_{c}}
$$

Um fluido teste é uma substância do banco de dados obtido por Reid et al. (1987). Este banco de dados contém colunas nesta ordem: a posição, a fórmula estrutural simplificada, o nome da substância (em inglês), a massa molecular, a temperatura do ponto de fusão (a pressão de uma atmosfera), a temperatura de saturação (a pressão de uma atmosfera), a 
temperatura crítica, o volume crítico, o fator de compressibilidade crítica, o fator de acentricidade de Pitzer e outras propriedades mais. Na Tabela D.1, mostramos uma única linha (posição 495) do banco de dados de Reid para a substância do iso-octano $\left(C_{8} H_{18}\right)$.

Tabela D.1 Propriedades do iso-octano (Reid et al. 1987).

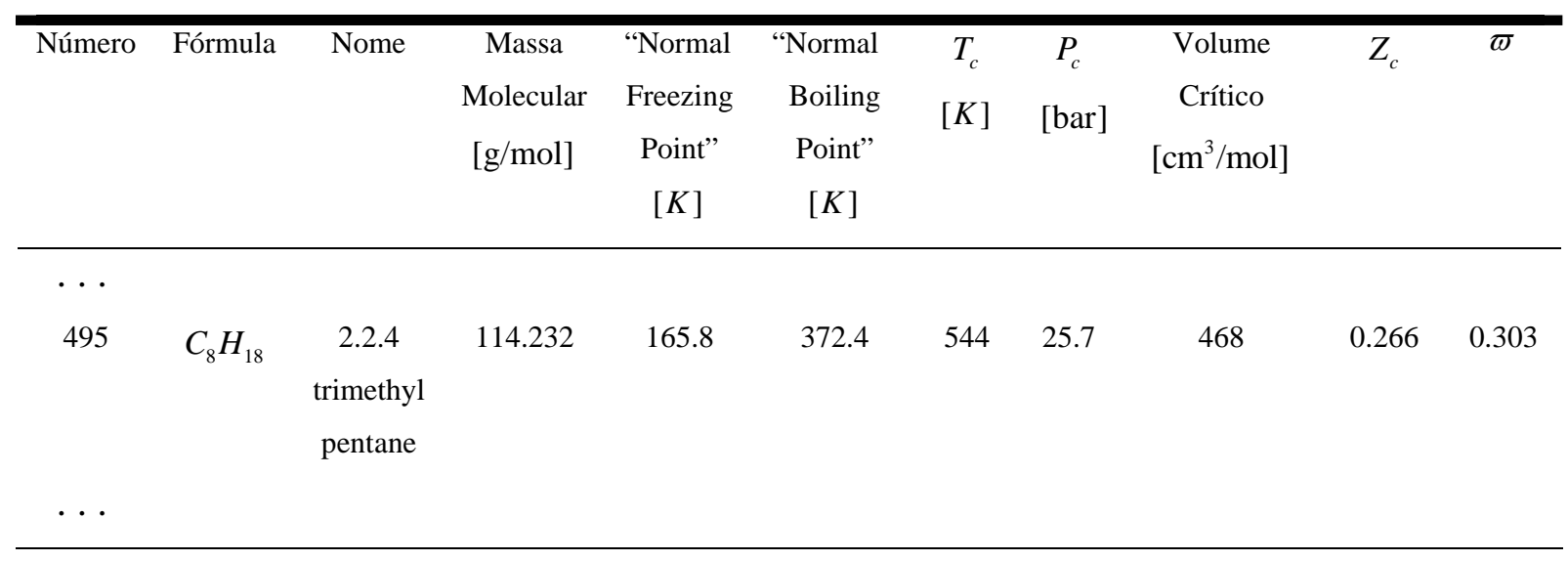

Para obter as propriedades de todas as substâncias do banco de dado de Reid se implementou uma sub-rotina chamada: REID, onde a variável de entrada é a fórmula ou o nome da substância.

O fator de compressibilidade para um fluido teste é definido por:

$$
Z_{\text {comp }}=\frac{p v}{R T}
$$

onde $R$ é a constante da substância e $v$ o volume específico. O fator de compressibilidade está relacionado com o fator de acentricidade da seguinte forma:

$$
Z_{\text {comp }}=Z_{\text {comp }}^{(0)}+\frac{\varpi}{\varpi^{(r)}}\left(Z_{\text {comp }}^{(r)}-Z_{\text {comp }}^{(0)}\right)
$$

Isolemos a temperatura e pressão de Eqs. (D.1) e (D.2) e substituíamos na Eq. (D.3), temos:

$$
Z_{\text {comp }}=\frac{p v}{R T}=\frac{P_{r d} P_{c} v}{R T_{r d} T_{c}}=\frac{P_{r d}}{T_{r d}} \frac{v}{R \frac{T_{c}}{P_{c}}}
$$

Definamos:

$$
v_{r d}^{\prime}=\frac{v}{R \frac{T_{c}}{P_{c}}}
$$


Substituindo Eq. (D.6) em Eq. (D.5), temos:

$$
Z_{\text {comp }}=\frac{P_{r d} v_{r d}^{\prime}}{T_{r d}}
$$

Dada duas propriedades termodinâmicas: $\{T, P\}$ queremos conhecer o volume específico, $v$. Para isto usamos a Equação de estado de Lee-Kesler, definida por:

$$
Z_{\text {comp }}=\frac{p_{r d} v_{r d}^{\prime}}{T_{r d}}=1+\frac{B_{L K}}{v_{r d}^{\prime}}+\frac{C_{L K}}{v_{r d}^{\prime 2}}+\frac{D_{L K}}{v_{r d}^{\prime 5}}+\frac{C_{4}}{T_{r d}^{3} v_{r d}^{\prime 2}}\left(\beta_{L K}+\frac{\gamma_{L K}}{v_{r d}^{\prime 2}}\right) \exp \left(-\frac{\gamma_{L K}}{v_{r d}^{\prime 2}}\right)
$$

onde os parâmetros $\left\{B_{L K}, C_{L K}, D_{L K}\right\}$ são dados por:

$$
\begin{aligned}
& B_{L K}=b_{1}-\frac{b_{2}}{T_{r d}}-\frac{b_{3}}{T_{r d}^{2}}-\frac{b_{4}}{T_{r d}^{3}} \\
& C_{L K}=c_{1}-\frac{c_{2}}{T_{r d}}+\frac{c_{3}}{T_{r d}^{3}} \\
& D_{L K}=d_{1}+\frac{d_{2}}{T_{r d}}
\end{aligned}
$$

e as constantes empíricas são dados na Tabela D.2, tanto para o fluido simples, quanto para o fluido de referência.

Tabela D.2 Constantes da Equação de Lee-Kesler.

\begin{tabular}{ccc}
\hline Constante & Fluido simples $(0)$ & Fluido de referência $(r)$ \\
\hline$b_{1}$ & 0,1181193 & 0,2026579 \\
$b_{2}$ & 0,265728 & 0,331511 \\
$b_{3}$ & 0,154790 & 0,027655 \\
$b_{4}$ & 0,030323 & 0,203488 \\
$c_{1}$ & 0,0236744 & 0,0313385 \\
$c_{2}$ & 0,0186984 & 0,0503618 \\
$c_{3}$ & 0,0 & 0,016901 \\
$c_{4}$ & 0,042724 & 0,041577 \\
$d_{1} \times 10^{4}$ & 0,155488 & 0,48736 \\
$d_{2} \times 10^{4}$ & 0,623689 & 0,0740336 \\
$\beta_{L K}$ & 0,65392 & 1,226 \\
$\gamma_{L K}$ & 0,060167 & 0,03754 \\
\hline
\end{tabular}


Dado o valor do fator de compressibilidade $Z_{\text {comp }}$ (calculado da Eq. (D.4)), se observa que a incógnita na equação algébrica não linear (Eq. (D.8)) é $v_{r d}^{\prime}$, pois $P_{r d}$ e $T_{r d}$ são conhecidos. Depois de resolver Eq. (D.8), por exemplo, pelo o método de Newton Raphson, a incógnita $v_{r d}^{\prime}$ é substituída na Eq. (D.6) , dessa forma temos conseguido achar o volume específico.

Uma sub-rotina é implementada para calcular as propriedades termodinâmicas nos estados seguintes:

\section{Líquido sub-resfriado}

Bifásico (líquido saturado / vapor saturado)

Vapor superaquecido com $T<T_{c}$

Gás

Vapor superaquecido com $T>T_{c}$

Esta sub-rotina chamada PROP, foi implementada por Angelo (2004) como parte de seu código computacional ShoWPhasT-2D v1.

Para o caso particular de líquidos altamente superaquecidos (estado (1)), a sub-rotina é chamada de: Equação_Estado_Lee-Kesler, conforme mostrada na Tabela D.3.

Tabela D.3 Sub-rotina para o cálculo da Equação de Estado de Lee-Kesler para o estado (1)

\begin{tabular}{ccc}
\hline Nome da sub-rotina & Notação & Descrição \\
\hline Equação_Estado_Lee-Kesler & $S E E L K:\{T, P\}\{v\}$ & $v:$ volume específico \\
\hline
\end{tabular}

Para o caso bifásico geramos uma tabela termodinâmica, mostrada na Tabela D.4, onde a primeira coluna é a temperatura de saturação, na segunda a pressão de saturação, na terceira é o volume específico do líquido saturado, na quarta é o volume específico do vapor saturado, na quinta a entalpia específica do líquido saturado, na sexta a entalpia específica do vapor saturado, na sétima a entropia específica do líquido saturado, e na última coluna a entropia específica do vapor saturado. A leitura desta tabela é armazenada numa matriz de ordem $(81 \times 8)$ a qual denotaremos por: Tab_iso $(i, j)$, onde o número de linhas, $N_{p}=81$. 
Tabela D.4 Tabela de propriedades de saturação do iso-octano $\left(C_{8} H_{18}\right)$.

\begin{tabular}{|c|c|c|c|c|c|c|c|}
\hline$T[\mathrm{~K}]$ & $P[\mathrm{~Pa}]$ & $v l\left[\mathrm{~m}^{3} \times \mathrm{kg}\right]$ & $v v\left[\mathrm{~m}^{3} \times \mathrm{kg}\right]$ & $h l[\mathrm{~J} / \mathrm{kg}]$ & $h v[\mathrm{~J} / \mathrm{kg}]$ & $s l[\mathrm{~J} / \mathrm{kg} \times \mathrm{K}]$ & $s v[\mathrm{~J} / \mathrm{kg} \times \mathrm{K}]$ \\
\hline 130,15 & 0,00000253 & 0,00120309 & 3739570157,67716 & $-230746,30$ & 156871,80 & $-1153,76$ & 1824,48 \\
\hline 135,15 & 0,00001204 & 0,00120531 & 817295192,60958 & $-224043,50$ & 160741,00 & $-1103,29$ & 1743,80 \\
\hline 140,15 & 0,00005059 & 0,00120813 & 201622394,67119 & $-217512,64$ & 164756,19 & $-1055,91$ & 1671,66 \\
\hline 145,15 & 0,00019063 & 0,00121131 & 55421734,59535 & $-211063,82$ & 168916,51 & $-1010,78$ & 1607,07 \\
\hline 150,15 & 0,00065108 & 0,00121504 & 16785601,44334 & $-204592,97$ & 173221,10 & $-967,04$ & 1549,21 \\
\hline 155,15 & 0,00203575 & 0,00121902 & 5547229,62376 & $-198100,00$ & 177669,13 & $-924,60$ & 1497,38 \\
\hline 160,15 & 0,00587729 & 0,00122338 & 1983347,64337 & $-191522,89$ & 182259,73 & $-882,98$ & 1450,98 \\
\hline 165,15 & 0,01578586 & 0,00122803 & 761480,89589 & $-184839,07$ & 186992,07 & $-841,99$ & 1409,49 \\
\hline 170,15 & 0,03970853 & 0,00123299 & 311886,72302 & $-178010,71$ & 191865,31 & $-801,37$ & 1372,45 \\
\hline 175,15 & 0,09409685 & 0,00123835 & 135482,63786 & $-171021,77$ & 196878,60 & $-761,01$ & 1339,48 \\
\hline 180,15 & 0,21115785 & 0,00124391 & 62097,66896 & $-163871,66$ & 202031,10 & $-720,89$ & 1310,21 \\
\hline 185,15 & 0,45081391 & 0,00124966 & 29893,31551 & $-156555,66$ & 207321,96 & $-680,97$ & 1284,35 \\
\hline 190,15 & 0,91949009 & 0,00125581 & 15052,05981 & $-149041,06$ & 212750,34 & $-641,06$ & 1261,60 \\
\hline 195,15 & 1,79833482 & 0,00126208 & 7898,46927 & $-141357,11$ & 218315,37 & $-601,33$ & 1241,73 \\
\hline 200,15 & 3,38390204 & 0,00126851 & 4305,06698 & $-133508,33$ & 224016,18 & $-561,77$ & 1224,51 \\
\hline 205,15 & 6,14461499 & 0,00127517 & 2430,04586 & $-125469,74$ & 229851,86 & $-522,27$ & 1209,74 \\
\hline 210,15 & 10,79643408 & 0,00128215 & 1416,70626 & $-117227,40$ & 235821,51 & $-482,75$ & 1197,24 \\
\hline 215,15 & 18,40101806 & 0,00128921 & 850,98127 & $-108832,83$ & 241924,15 & $-443,46$ & 1186,83 \\
\hline 220,15 & 30,48927332 & 0,00129638 & 525,50543 & $-100279,72$ & 248158,78 & $-404,35$ & 1178,38 \\
\hline 225,15 & 49,21253264 & 0,00130379 & 332,95126 & $-91544,13$ & 254524,33 & $-365,31$ & 1171,75 \\
\hline 230,15 & 77,52271750 & 0,00131140 & 216,04142 & $-82637,61$ & 261019,69 & $-326,40$ & 1166,79 \\
\hline 235,15 & 119,38176791 & 0,00131909 & 143,32502 & $-73591,37$ & 267643,64 & $-287,73$ & 1163,41 \\
\hline 240,15 & 179,99943768 & 0,00132702 & 97,06733 & $-64370,17$ & 274394,91 & $-249,15$ & 1161,49 \\
\hline 245,15 & 266,09732836 & 0,00133510 & 67,01669 & $-54999,08$ & 281272,13 & $-210,77$ & 1160,93 \\
\hline 250,15 & 386,19585093 & 0,00134325 & 47,10794 & $-45498,66$ & 288273,81 & $-172,65$ & 1161,64 \\
\hline 255,15 & 550,91973554 & 0,00135165 & 33,67391 & $-35833,38$ & 295398,38 & $-134,66$ & 1163,53 \\
\hline 260,15 & 773,31681751 & 0,00136000 & 24,45169 & $-26075,31$ & 302644,16 & $-97,05$ & 1166,53 \\
\hline 265,15 & 1069,18416109 & 0,00136863 & 18,01788 & $-16151,29$ & 310009,35 & $-59,54$ & 1170,56 \\
\hline 270,15 & 1457,39516927 & 0,00137753 & 13,46092 & $-6065,10$ & 317492,07 & $-22,15$ & 1175,55 \\
\hline 275,15 & 1960,22117984 & 0,00138640 & 10,18707 & 4104,51 & 325090,29 & 14,85 & 1181,43 \\
\hline 280,15 & 2603,64115984 & 0,00139538 & 7,80334 & 14392,68 & 332801,90 & 51,59 & 1188,16 \\
\hline 285,15 & 3417,63346159 & 0,00140463 & 6,04572 & 24833,54 & 340624,71 & 88,21 & 1195,66 \\
\hline 290,15 & 4436,44416373 & 0,00141400 & 4,73427 & 35387,00 & 348556,41 & 124,56 & 1203,90 \\
\hline 295,15 & 5698,82725057 & 0,00142352 & 3,74470 & 46056,55 & 356594,60 & 160,67 & 1212,81 \\
\hline 300,15 & 7248,25274013 & 0,00143316 & 2,99008 & 56833,56 & 364736,83 & 196,51 & 1222,34 \\
\hline 305,15 & 9133,07981137 & 0,00144308 & 2,40884 & 67750,94 & 372980,56 & 232,21 & 1232,47 \\
\hline 310,15 & 11406,69296219 & 0,00145310 & 1,95689 & 78770,57 & 381323,20 & 267,64 & 1243,14 \\
\hline 315,15 & 14127,60021294 & 0,00146333 & 1,60232 & 89901,11 & 389762,08 & 302,83 & 1254,32 \\
\hline 320,15 & 17359,49332186 & 0,00147366 & 1,32177 & 101129,44 & 398294,52 & 337,76 & 1265,97 \\
\hline 325,15 & 21171,27087156 & 0,00148412 & 1,09801 & 112453,81 & 406917,78 & 372,42 & 1278,05 \\
\hline 330,15 & 25637,02589830 & 0,00149488 & 0,91817 & 123910,08 & 415629,08 & 406,94 & 1290,53 \\
\hline 335,15 & 30836,00045257 & 0,00150592 & 0,77259 & 135489,86 & 424425,62 & 441,28 & 1303,39 \\
\hline 340,15 & 36852,51009253 & 0,00151699 & 0,65393 & 147136,53 & 433304,57 & 475,29 & 1316,59 \\
\hline 345,15 & 43775,84181609 & 0,00152845 & 0,55657 & 158928,62 & 442263,08 & 509,20 & 1330,11 \\
\hline 350,15 & 51700,12933459 & 0,00153998 & 0,47619 & 170788,73 & 451298,27 & 542,80 & 1343,91 \\
\hline 355,15 & 60724,20988461 & 0,00155190 & 0,40944 & 182788,18 & 460407,24 & 576,29 & 1357,98 \\
\hline 360,15 & 70951,46697332 & 0,00156398 & 0,35370 & 194873,00 & 469587,08 & 609,52 & 1372,29 \\
\hline 365,15 & 82489,66356457 & 0,00157636 & 0,30689 & 207070,42 & 478834,82 & 642,57 & 1386,83 \\
\hline 370,15 & 95450,77025117 & 0,00158894 & 0,26739 & 219353,64 & 488147,47 & 675,39 & 1401,56 \\
\hline 375,15 & 109950,79293340 & 0,00160194 & 0,23389 & 231768,06 & 497522,01 & 708,08 & 1416,47 \\
\hline 380,15 & 126109,60444919 & 0,00161525 & 0,20535 & 244286,58 & 506955,35 & 740,59 & 1431,55 \\
\hline 385,15 & 144050,78448912 & 0,00162875 & 0,18092 & 256884,26 & 516444,34 & 772,85 & 1446,77 \\
\hline 390,15 & 163901,47199302 & 0,00164271 & 0,15992 & 269607,43 & 525985,75 & 804,98 & 1462,11 \\
\hline 395,15 & 185792,23407620 & 0,00165710 & 0,14180 & 282448,35 & 535576,27 & 836,97 & 1477,56 \\
\hline 400,15 & 209856,95538532 & 0,00167183 & 0,12610 & 295385,91 & 545212,45 & 868,77 & 1493,11 \\
\hline 405,15 & 236232,75164836 & 0,00168702 & 0,11244 & 308438,00 & 554890,73 & 900,43 & 1508,73 \\
\hline 410,15 & 265059,91107263 & 0,00170272 & 0,10052 & 321604,13 & 564607,36 & 931,94 & 1524,41 \\
\hline 415,15 & 296481,86717226 & 0,00171884 & 0,09008 & 334874,02 & 574358,40 & 963,28 & 1540,14 \\
\hline 420,15 & 330645,20658681 & 0,00173549 & 0,08091 & 348253,85 & 584139,68 & 994,48 & 1555,91 \\
\hline 425,15 & 367699,71550078 & 0,00175276 & 0,07282 & 361754,94 & 593946,73 & 1025,55 & 1571,69 \\
\hline 430,15 & 407798,46841040 & 0,00177069 & 0,06566 & 375375,37 & 603774,76 & 1056,50 & 1587,48 \\
\hline 435,15 & 451097,96323284 & 0,00178923 & 0,05931 & 389107,38 & 613618,59 & 1087,31 & 1603,25 \\
\hline 440,15 & 497758,30714485 & 0,00180857 & 0,05367 & 402968,66 & 623472,52 & 1118,02 & 1618,99 \\
\hline 445,15 & 547943,45811635 & 0,00182876 & 0,04863 & 416955,72 & 633330,32 & 1148,63 & 1634,70 \\
\hline 450,15 & 601821,52792598 & 0,00184976 & 0,04412 & 431059,88 & 643185,05 & 1179,11 & 1650,34 \\
\hline 455,15 & 659565,15359271 & 0,00187184 & 0,04007 & 445307,90 & 653028,95 & 1209,52 & 1665,90 \\
\hline 460,15 & 721351,94574733 & 0,00189507 & 0,03643 & 459696,20 & 662853,23 & 1239,87 & 1681,37 \\
\hline 465,15 & 787365,02467298 & 0,00191948 & 0,03315 & 474218,66 & 672647,85 & 1270,13 & 1696,72 \\
\hline 470,15 & 857793,65782358 & 0,00194532 & 0,03018 & 488893,55 & 682401,23 & 1300,34 & 1711,93 \\
\hline 475,15 & 932834,01698360 & 0,00197282 & 0,02749 & 503729,44 & 692099,85 & 1330,52 & 1726,96 \\
\hline 480,15 & 1012690,07949176 & 0,00200219 & 0,02504 & 518738,25 & 701727,69 & 1360,69 & 1741,80 \\
\hline 485,15 & 1097574,70714167 & 0,00203364 & 0,02280 & 533915,57 & 711265,54 & 1390,85 & 1756,41 \\
\hline 490,15 & 1187710,95021758 & 0,00206754 & 0,02076 & 549281,68 & 720689,96 & 1421,02 & 1770,73 \\
\hline 495,15 & 1283333,64563149 & 0,00210452 & 0,01888 & 564867,41 & 729971,74 & 1451,28 & 1784,72 \\
\hline 500,15 & 1384691,41278453 & 0,00214499 & 0,01715 & 580677,02 & 739073,67 & 1481,62 & 1798,32 \\
\hline 505,15 & 1492049,20910492 & 0,00218999 & 0,01555 & 596754,97 & 747947,43 & 1512,13 & 1811,43 \\
\hline 510,15 & 1605691,71068927 & 0,00224047 & 0,01406 & 613128,54 & 756527,34 & 1542,85 & 1823,95 \\
\hline 515,15 & 1725927,97927313 & 0,00229836 & 0,01266 & 629868,53 & 764720,75 & 1573,92 & 1835,69 \\
\hline 520,15 & 1853098,27861996 & 0,00236634 & 0,01134 & 647063,71 & 772388,99 & 1605,49 & 1846,43 \\
\hline 525,15 & 1987584,82037641 & 0,00244904 & 0,01008 & 664859,97 & 779310,52 & 1637,82 & 1855,76 \\
\hline 530,15 & 2129830,63923724 & 0,00255522 & 0,00884 & 683524,67 & 785080,82 & 1671,40 & 1862,96 \\
\hline
\end{tabular}




\section{D.2 Interpolação das Propriedades Termodinâmicas}

Conseguida a tabela das propriedades termodinâmicas na curva de saturação para o isooctano, procedemos a implementação uma sub-rotina que interpole uma das três propriedades termodinâmicas (temperatura de saturação, pressão de saturação ou entropia específica do líquido saturado), consideradas como variáveis de entrada. O código desta sub-rotina foi implementado seguindo a teoria exposta no artigo de Figueiredo et al. (2007). O nome desta sub-rotina é: Interpolação_Lee-Kesler, e para o caso onde a variável de entrada é a temperatura de saturação é mostrada na Tabela D.5, onde a primeira chave indica as variáveis de entrada e a segunda chave as variáveis de saída.

Tabela D.5 Sub-rotina: Interpolação_Lee-Kesler.

\begin{tabular}{|c|c|c|}
\hline Nome da sub-rotina & Notação & Descrição \\
\hline Interpolação_Lee-Kesler & SInterpolLK : $\left\{T_{\text {sat }}\right\}\left\{P_{\text {sat }}, v l_{\text {sat }}, v v_{\text {sat }}, h l_{\text {sat }}, h v_{\text {sat }}, s l_{\text {sat }}, s v_{\text {sat }}\right\}$ & $\begin{array}{l}\text { Variável de } \\
\text { entrada: } T_{\text {sat }}\end{array}$ \\
\hline
\end{tabular}

- $\quad$ Algoritmo da sub-rotina: Interpolação_Lee-Kesler.

O algoritmo em pseudocódigo da sub-rotina: Interpolação_Lee-Kesler é mostrada na Fig. D.1. Este algoritmo usa principalmente quatro sub-rotina: Cálculo_Psat, Cálculo_vlsat_vvsat, Cálculo_hlsat_hvsat e Cálculo_slsat_svsat, dadas na Tabela D.6.

Tabela D.6 Sub-rotinas que compõem a rotina: Interpolação_Lee-Kesler.

\begin{tabular}{|c|c|c|}
\hline Nome da sub-rotina & Notação & Descrição \\
\hline Cálculo_Psat & SCalPsat: $\left\{T_{\text {sat }}, T_{1}, T_{2}, P_{1}, P_{2}\right\}\left\{P_{\text {sat }}\right\}$ & $\begin{array}{l}\text { Dois pontos de } \\
\text { interpolação }\end{array}$ \\
\hline Cálculo_vlsat_vvsat & SCalvlsat \& vvsat $:\left\{\begin{array}{l}T_{\text {sat }}, T_{r}, T_{r 1}, T_{r 2}, T_{1}, T_{2}, P_{\text {sat }}, \\
P_{1}, P_{2}, v l_{1}, v l_{2}, v v_{1}, v v_{2}\end{array}\right\}\left\{v l_{\text {sat }}, v v_{\text {sat }}\right\}$ & $\begin{array}{l}\text { Dois pontos de } \\
\text { interpolação }\end{array}$ \\
\hline Cálculo_hlsat_hvsat & SCalhlsat \& hvsat : $\left\{\begin{array}{l}T_{s a t}, T_{r}, T_{r 1}, T_{r 2}, T_{1}, \\
T_{2}, h l_{1}, h l_{2}, h v_{1}, h v_{2}\end{array}\right\}\left\{h l_{s a t}, h v_{s a t}\right\}$ & $\begin{array}{l}\text { Dois pontos de } \\
\text { interpolação }\end{array}$ \\
\hline Cálculo_slsat_svsat & SCalslsat \& svsat : $\left\{\begin{array}{l}T_{\text {sat }}, T_{1}, T_{2}, T_{3}, h l v, \\
s l_{1}, s l_{2}, s l_{3}, s v_{1}\end{array}\right\}\left\{s l_{\text {sat }}, s v_{\text {sat }}\right\}$ & $\begin{array}{l}\text { Três pontos de } \\
\text { interpolação }\end{array}$ \\
\hline
\end{tabular}

É importante indicar que o código ShoWPhasT-2D v1 (ANGELO, 2004) usa a solução da Equação de Lee-Kesler (ver Eq. (D.8)) cada vez que é chamado para computar as 
propriedades termodinâmicas. Dessa forma usa muito tempo computacional, pois, em cada nó do domínio bifásico é resolvida a Equação de Lee-Kesler pelo método de Newton-Raphson. $\mathrm{Na}$ necessidade de se reduzir o custo computacional se implementou a sub-rotina Interpolação_Lee-Kesler que usa a tabela das propriedades termodinâmicas (veja Tabela D.4) e logo interpola os valores procurados, usando fórmulas simples de cálculo direto. Reduzindo dessa forma enormemente o custo computacional.

Entrada: $\left\{T_{\text {sat }}\right\}$

Saída: $\left\{P_{s a t}, v l_{s a t}, v v_{s a t}, h l_{s a t}, h v_{s a t}, s l_{s a t}, s v_{s a t}\right\}$

1. Leitura da tabela de propriedades termodinâmicas

2. Leitura da sub-rotina REID para obter a $T_{c}$

3. Armazenando em uma matriz a primeira coluna da tabela termodinâmica: $\operatorname{Tab}(i, 1), \quad i=\overline{1, N_{p}}$

4. Satisfazendo a condição de que $T_{\text {sat }}$ está entre $\operatorname{Tab}(1,1)$ e $\operatorname{Tab}\left(N_{p}-1,1\right)$

5. Localização de três temperaturas que contenha a $T_{\text {sat }}$, ou seja, os pontos: $\{i, i+1, i+2\}$

6. Definamos:

$$
\begin{aligned}
& T_{n}=\text { Tab_iso }(k, 1) h l_{n}=\text { Tab_iso }(k, 5) \\
& P_{n}=\text { Tab_iso }(k, 2) h v_{n}=\text { Tab_iso }(k, 6) \\
& v l_{n}=\text { Tab_iso }(k, 3) s l_{n}=\text { Tab_iso }(k, 7) \\
& v v_{n}=\text { Tab_iso }(k, 4) s v_{n}=\text { Tab_iso }(k, 8) \\
& \text { onde } n=\overline{1,3} \text { (fixo), e } k=\{i, i+1, i+2\}
\end{aligned}
$$

7. Uso SCalPsat : $\left\{T_{\text {sat }}, T_{1}, T_{2}, P_{1}, P_{2}\right\}\left\{P_{\text {sat }}\right\}$

8. Definamos: $T_{r}=\frac{T_{s a t}}{T_{c}} ; T_{r 1}=\frac{T_{1}}{T_{c}} ; T_{r 2}=\frac{T_{2}}{T_{c}}$

9. Uso SCalvlsat \& vvsat: $\left\{\begin{array}{l}T_{s a t}, T_{r}, T_{r 1}, T_{r 2}, T_{1}, T_{2}, P_{s a t}, \\ P_{1}, P_{2}, v l_{1}, v l_{2}, v v_{1}, v v_{2}\end{array}\right\}\left\{v l_{s a t}, v v_{s a t}\right\}$

10. Uso SCalhlsat \& hvsat: $\left\{\begin{array}{l}T_{s a t}, T_{r}, T_{r 1}, T_{r 2}, T_{1}, \\ T_{2}, h l_{1}, h l_{2}, h v_{1}, h v_{2}\end{array}\right\}\left\{h l_{s a t}, h v_{s a t}\right\}$

11. Uso SCalslsat \& svsat: $\left\{\begin{array}{l}T_{\text {sat }}, T_{1}, T_{2}, T_{3}, h l v, \\ s l_{1}, s l_{2}, s l_{3}, s v_{1}\end{array}\right\}\left\{s l_{s a t}, s v_{s a t}\right\}$

12. Portanto temos calculado: $\left\{P_{\text {sat }}, v l_{\text {sat }}, v v_{\text {sat }}, h l_{\text {sat }}, h v_{\text {sat }}, s l_{\text {sat }}, s v_{\text {sat }}\right\}$

Figura D.1 Algoritmo em pseudocódigo da sub-rotina Interpolação de Lee-Kesler. 